Florida International University

FIU Digital Commons

FIU Electronic Theses and Dissertations

University Graduate School

6-25-2019

\title{
Functionalized Aptamers for Detection of Small-Molecule Targets
}

Haixiang Yu

Florida International University, hyu010@fiu.edu

Follow this and additional works at: https://digitalcommons.fiu.edu/etd

Part of the Analytical Chemistry Commons, and the Biochemistry Commons

\section{Recommended Citation}

Yu, Haixiang, "Functionalized Aptamers for Detection of Small-Molecule Targets" (2019). FIU Electronic Theses and Dissertations. 4249.

https://digitalcommons.fiu.edu/etd/4249

This work is brought to you for free and open access by the University Graduate School at FIU Digital Commons. It has been accepted for inclusion in FIU Electronic Theses and Dissertations by an authorized administrator of FIU Digital Commons. For more information, please contact dcc@fiu.edu. 


\section{FLORIDA INTERNATIONAL UNIVERSITY}

Miami, Florida

\section{FUNCTIONALIZED APTAMERS FOR DETECTION \\ OF SMALL-MOLECULE TARGETS}

A dissertation submitted in partial fulfillment of

the requirements for the degree of

DOCTOR OF PHILOSOPHY

in

BIOCHEMISTRY

by

Haixiang $\mathrm{Yu}$

2019 
To: Dean Michael R. Heithaus

College of Arts, Sciences and Education

This dissertation, written by Haixiang Yu, and entitled Functionalized Aptamers for Detection of Small-Molecule Targets, having been approved in respect to style and intellectual content, is referred to you for judgment.

We have read this dissertation and recommend that it be approved.

$\begin{array}{r}\hline \text { Prem Chapagain } \\ \hline \text { Ylexander Mebel } \\ \hline \text { Yi Xiao, Major Professor }\end{array}$

Date of Defense: June 25, 2019

The dissertation of Haixiang $\mathrm{Yu}$ is approved.

Dean Michael R. Heithaus

College of Arts, Sciences and Education

Andrés G. Gil

Vice President for Research and Economic Development and Dean of the University Graduate School

Florida International University, 2019 
(C) Copyright 2019 by Haixiang Yu

All rights reserved. 


\section{DEDICATION}

I dedicate this work to my parents Boyang Yu and Xuming Liu, and my grandmother Lijuan Xia with my deepest love. 


\section{ACKNOWLEDGMENTS}

No accomplishment is achieved by a single pair of hands. My dissertation could have not been possible without the cooperation and support of numerous people. I want to express my most sincere appreciation to those who have helped me during my six years of study at FIU in my humble acknowledgment below.

First and foremost, I would like to express my utmost gratitude to my major professor Dr. Yi Xiao, for her continuous effort and care throughout my Ph.D. years. Dr. Xiao has armed me with knowledge and skills, nurtured me with every opportunity and resource, ignited my dreams, and enlightened my way to future success. She has been a role model that encouraged me to conquer any obstacle, and is a beacon that gives me strength and persistence to overcome the darkest times. This artist carved and filed me into the current state I am now. I owe all my achievements to Dr. Xiao.

I am most grateful to my dissertation committee, Dr. Prem Chapagain, Dr. Yuan Liu, and Dr. Alexander Mebel for their constant guidance and support during my graduate research and career development. They have made time for me whenever I needed help and have provided numerous valuable advices during the past six years. I would like to give special thanks to Dr. Liu, who is also the biochemistry graduate program director, for keep tracking my graduation progress during the years. I would also like to thank Dr. Xiaotang Wang, the former graduate program director, who admitted me into this great program and provided considerable help and support on my research and life in the past six years.

I would like to acknowledge the Graduate Research Fellowship (2015-R2-CX-0034) from the National Institute of Justice, Office of Justice Programs, U.S. Department of 
Justice, which not only funded my stipend and research between 2015 and 2018, but also supported me for training and presenting at several national research conferences throughout the years. I would like to acknowledge the Department of Chemistry and Biochemistry and Dissertation Year Fellowship from University Graduate School at FIU for the financial support during my Ph.D. research. I would also like to thank the Graduate and Professional Student Committee and the dean's office of the College of Arts, Sciences and Education at Florida International University for travel funding. These supports were essential for me to acquire this Ph.D.

I would like to thank all current and formal lab mates for their support. Particularly, I would like to thank Obtin Alkhamis, Bhargav Guntupalli, Juan Canoura, Pingping Liang, Yingzhu Liu, Yingping Luo, Zongwen Wang, Weijuan Yang for their direct contribution to my dissertation research. I could not accomplish my research without your help.

I would also like to spread my gratitude to our collaborators for their generous help with my research. I would like to Dr. Milan Stojanovic and Dr. Kyung-Ae Yang at Columbia University for their training and guidance on aptamer isolation; Dr. Xinhui Lou and Dr. Shi Xu at Capital Normal University for their guidance on isothermal titration calorimetry; Dr. Yi Lu and Kang Yong Loh at University of Illinois Urbana-Champaign for their help for nanomaterial synthesis and characterization; and Dr. Paul Sharp at the FIU Department of Biological Sciences for assistance with the DNA sequencing process.

I would also like to thank the faculty and staff members of the Department of Chemistry and Biochemistry for their help during my Ph.D. study. Special thanks to Magali Autie, Jackelyn Marcos, and Lilia San Miguel, who help me the most over the years. 
Last but not least, I would like to express my very profound gratitude to my parents Boyang Yu and Xuming Liu and my grandmother Lijuan Xia for their endless support, care, and encouragement throughout my years of study. They have been giving me strength for me to overcome even the hardest of times. I would also like to thank my other family members and friends for their support and encouragement; I must apologize as I could not mention each personally one by one. 


\section{ABSTRACT OF THE DISSERTATION \\ FUNCTIONALIZED APTAMERS FOR DETECTION \\ OF SMALL-MOLECULE TARGETS}

by

Haixiang Yu

Florida International University, 2019

Miami, Florida

Professor Yi Xiao, Major Professor

Aptamers have recently gained considerable attention for small-molecule detection in diverse applications such as drug identification, medical diagnostics, and environmental monitoring. However, the performance of aptamer-based sensors has been greatly limited by the low target affinity and responsiveness of small-molecule binding aptamers. This dissertation describes several novel aptamer engineering and isolation strategies to remedy this problem. Specifically, we first develop a generally applicable strategy to engineer split aptamers containing two binding domains termed cooperative-binding split aptamers (CBSAs). CBSAs exhibit higher target binding affinity and are far more responsive in terms of target-induced split aptamer assembly compared to single-domain parent split aptamers from which they are derived. Using a cocaine-binding CBSA, we achieve specific fluorescence detection of as low as $50 \mathrm{nM}$ cocaine in $10 \%$ saliva within 15 minutes. We then develop a general approach for creating rapid and sensitive CBSAbased enzyme-assisted target recycling (EATR)-amplified small-molecule sensors for sensitive target detection. Using this strategy, we develop a fluorescence assay for dehydroisoandrosterone-3-sulfate which achieves 100-fold enhanced target sensitivity 
relative to a non-EATR-based assay, and a colorimetric assay for visual detection of lowmicromolar concentrations of cocaine. To simplify the sensor development process, we establish a novel and simple SELEX strategy for directly isolating aptamers with intrinsic dye-displacement functionality which transduce target-binding events into a change of dye absorbance. As a demonstration, we isolate an aptamer against the synthetic cathinone 3,4-methylenedioxypyrovalerone (MDPV) that can detect MDPV at concentrations as low as $300 \mathrm{nM}$ in a label-free, rapid, and simple dye-displacement assay. To further control the target-binding spectra of aptamers, we employ a novel parallel-and-serial SELEX strategy to isolate an aptamer binding 12 synthetic cathinones with nanomolar affinity but not 11 non-target compounds that are closely related in structure. Using this aptamer, instantaneous visual detection of synthetic cathinones at nanomolar concentrations in biological samples is achieved. In summary, this work demonstrates the great potential of novel aptamer engineering and isolation strategies in generating functional signal-reporting aptamers for sensitive small molecule detection. Importantly, the strategies described here are generalizable and can be used to develop aptamer-based assays for virtually any small-molecule targets. 


\section{TABLE OF CONTENTS}

CHAPTER

PAGE

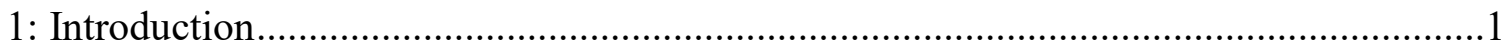

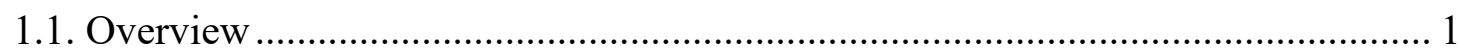

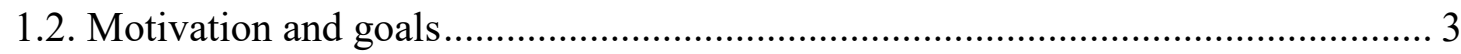

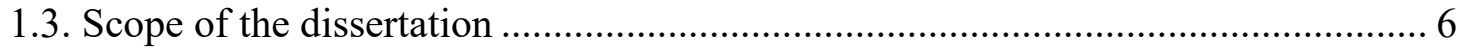

2: Background and Literature Review …………………...........................................

2.1. Aptamers and their use as affinity reagents for small-molecule targets ................ 7

2.1.1 Advantages of aptamers as biorecognition elements for small molecule

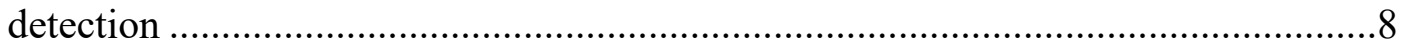

2.2. Isolation of small-molecule-binding aptamers via SELEX …………………........ 11

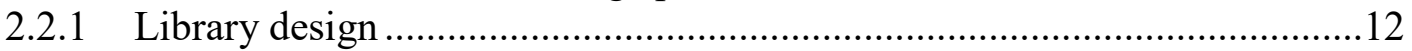

2.2.2 Strand separation strategies for SELEX …………......................................13

2.2.3 Aptamer enrichment by PCR and regeneration of single-stranded libraries.....

2.2.4 Common selection strategies for control affinity and specificity of aptamers..

2.3. Aptamer-based sensors for small molecule detection ........................................... 20

2.3.1 Sensors based on target-induced conformational changes.............................21

2.3.2 Sensors that do not require target-induced conformational change..............27

2.4. Gold nanoparticles (AuNPs) and their use in aptamer-based sensors................... 30

2.4.1 Synthesis and characterization of citrate-capped AuNPs ...............................31

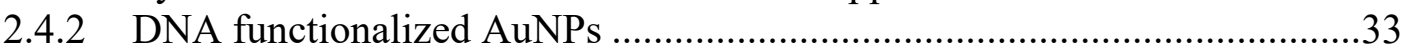

2.4.3 Aptamer-based assays employing AuNPs for small molecule detection......36

2.5. EATR-based signal amplification ...................................................................... 39

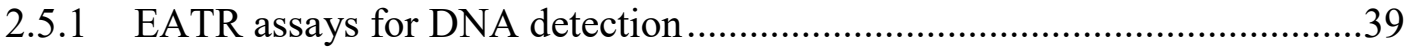

2.5.2 Aptamer-based EATR assays for small-molecular detection ........................41

2.6. Small molecule targets used in this work............................................................. 42

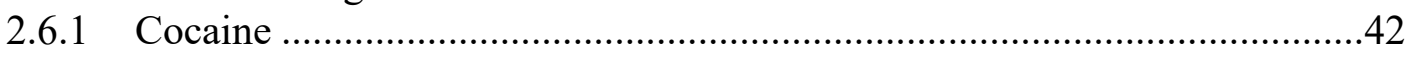

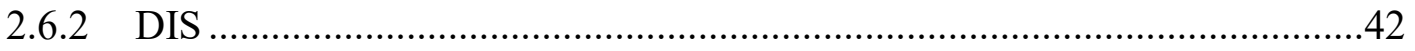

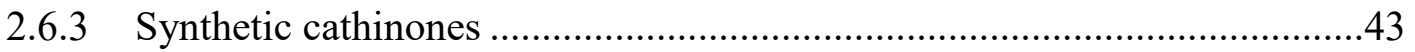

3: A Cooperative-Binding Split Aptamer Assay for Rapid, Specific and Ultra-Sensitive

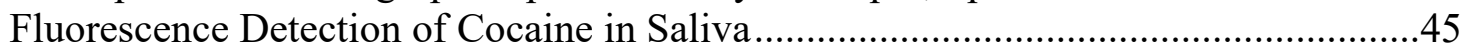

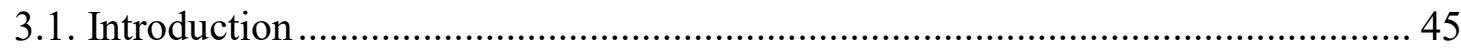

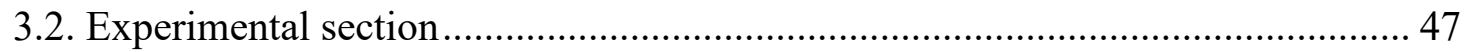

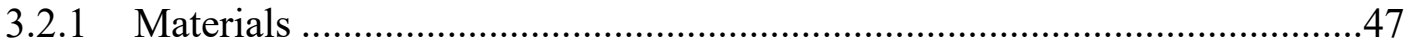

3.2.2 Characterization of split aptamer assembly using ATMND ........................48

3.2.3 Isothermal titration calorimetry (ITC) experiments.....................................49

3.2.4 Fluorophore/quencher-modified CBSA-5325, -5335, or -5335-GT for

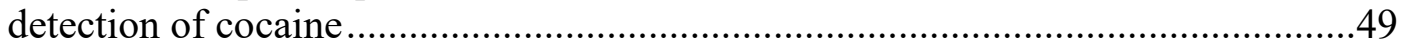

3.2.5 Assessing saliva matrix and dilution effects on the CBSA-5335-based fluorophore/quencher assay ..............................................................................50 
3.2.6 Determining the sensitivity and dynamic range of CBSA-5335-based

fluorophore/quencher assay in saliva ............................................................51

3.2.7 Determining the target specificity of CBSA-5335-based

fluorophore/quencher assay in saliva ...............................................................52

3.2.8 Determining the precision and bias of CBSA-5335-based

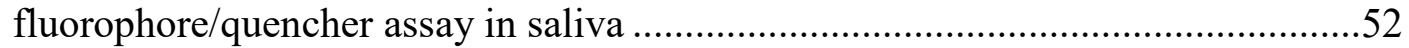

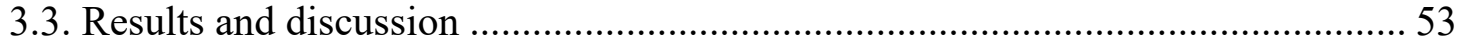

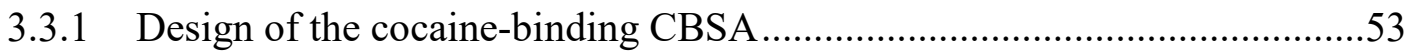

3.3.2 Characterizing cocaine-induced CBSA assembly ..................................54

3.3.3 Importance of dual-binding domain of CBSA for target-induced

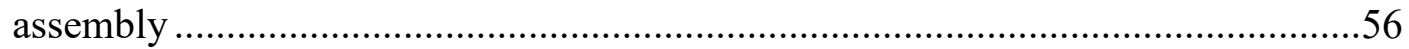

3.3.4 Confirmation of cooperative target binding of CBSA and fabricating an ultra-sensitive CBSA-based fluorophore/quencher assay ..................................61

3.3.5 Validation of the CBSA-5335-based fluorophore/quencher assay for

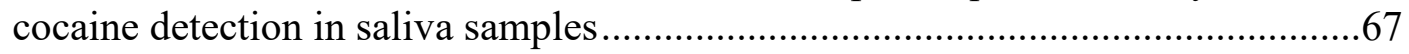

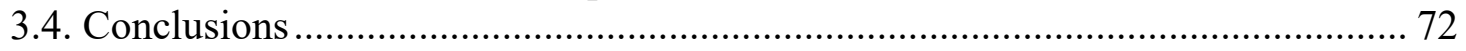

4: Rapid and Sensitive Detection of Small-Molecule Targets Using Cooperative Binding Split Aptamers and Enzyme-Assisted Target Recycling.............................74

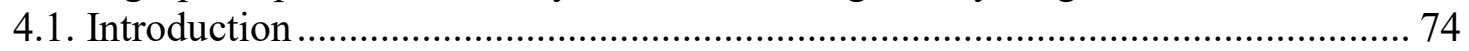

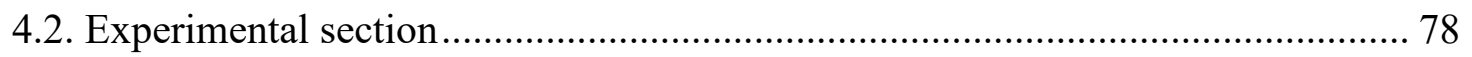

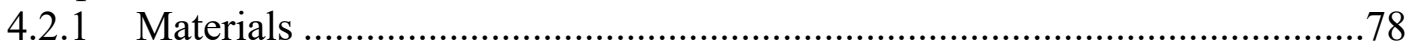

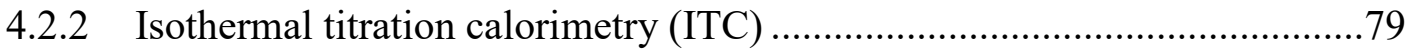

4.2.3 Determining binding affinity of DIS-CBSA-4536, DIS-SA-536 and DIS-

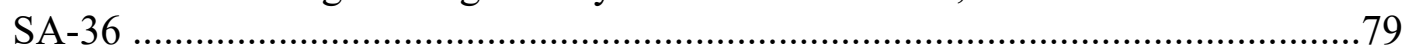

4.2.4 Exo III digestion and gel electrophoresis analysis.................................80

4.2.5 CBSA-based, EATR-amplified fluorescence assay for detection of DIS....80

4.2.6 Synthesis of gold nanoparticles (AuNPs) modified with cocaine-binding

CBSA short fragment ............................................................................... 81

4.2.7 Quantitation of the surface coverage of CBSA short fragments on AuNPs

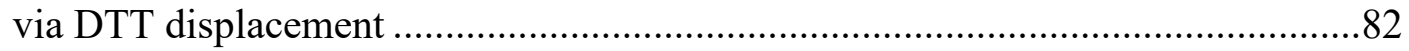

4.2.8 CBSA-based, colorimetric EATR assay for detection of cocaine ...............82

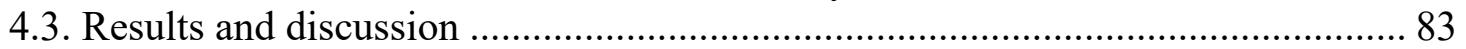

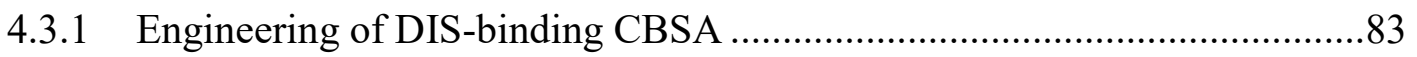

4.3.2 Design of a CBSA-based EATR-amplified fluorescence assay ..................85

4.3.3 Exo III efficiently mediates EATR of DIS-CBSA-4536 in solution ...........88

4.3.4 Sensitive DIS Detection in biofluid samples using CBSA-based EATRamplified fluorescence assay ................................................................. 90

4.3.5 Design of the CBSA-based colorimetric assay employing EATRmediated AuNP aggregation ................................................................... 92

4.3.6 EATR of CBSA mediated by AP endonuclease of Exo III instead of its

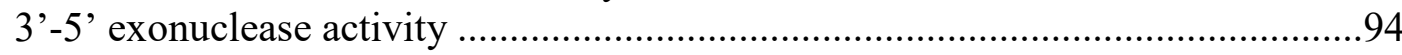

4.3.7 DNA surface coverage and the length of COC-SF affect EATR-mediated AuNP aggregation ................................................................................ 97

4.3.8 Visual detection of cocaine using CBSA-based EATR-amplified colorimetric assay..... 
5: In Vitro Isolation of Small-Molecule-Binding Aptamers with Intrinsic Dye-

Displacement Functionality.....

5.1. Introduction

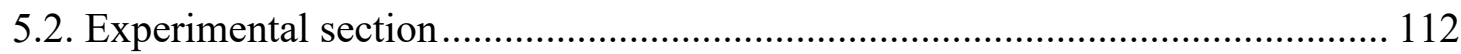

5.2.1 Materials

5.2.2 SELEX procedure.

5.2.3 Cloning and sequencing

5.2.4 Characterization of target displacement of Cy7 from TWJ-structured aptamers.

5.2.5 Characterization of binding of $\mathrm{Cy} 7$ to TWJ pool.

5.2.6 Continuous variation experiment (Job plot)

5.2.7 Development of a target elution assay to assess pool affinity and target specificity

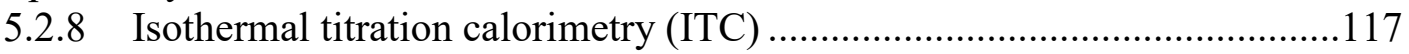

5.2.9 Strand-displacement fluorescence assay for detection of MDPV.............117

5.2.10 Cy7 displacement assay for detection of MDPV ...............................118

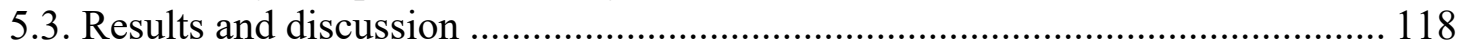

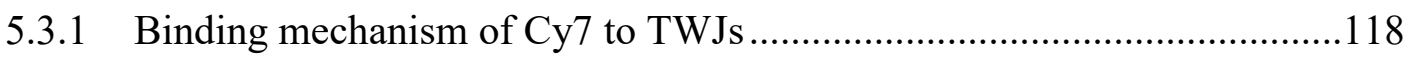

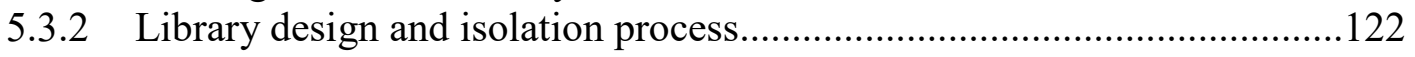

5.3.3 Aptamer characterization and development of the label-free Cy7-

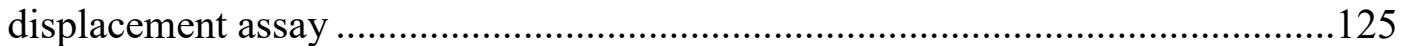

5.3.4 Comparison of Cy7-displacement and strand-displacement assays ..........130

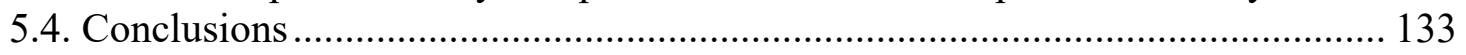

6: In Vitro Isolation of Class-Specific Oligonucleotide-Based Small-Molecule

Receptors

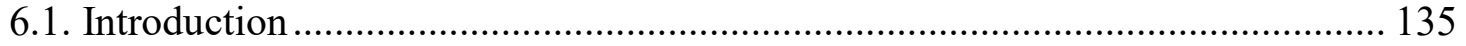

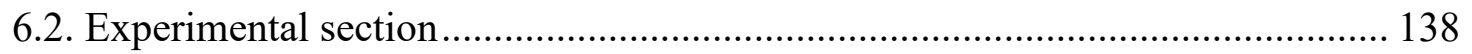

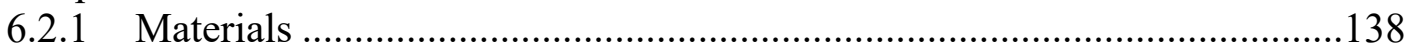

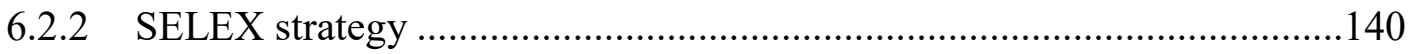

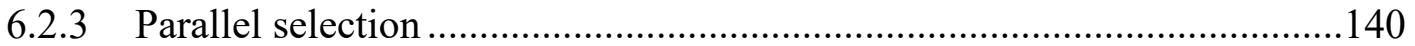

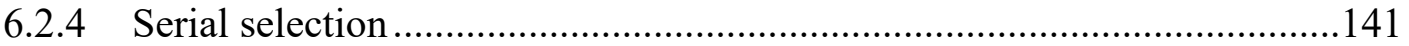

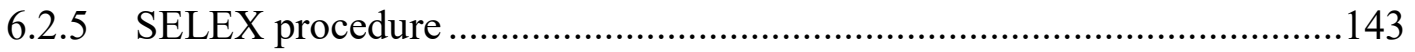

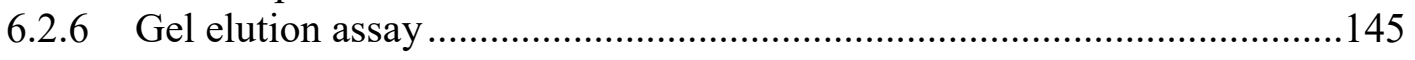

6.2.7 High throughput sequencing (HTS) analysis of the aptamer isolation

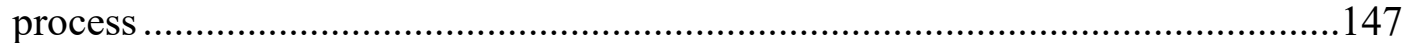

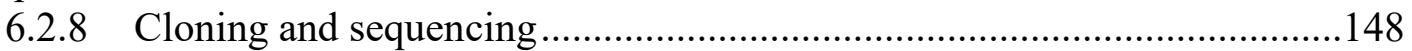

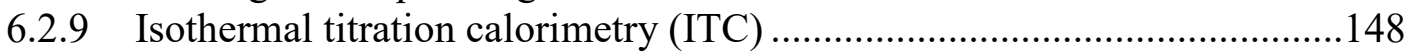

6.2.10 Characterization of Cy7 binding to SCA2.1 ....................................150

6.2.11 Cy7-displacement assay for colorimetric detection of synthetic cathinones.

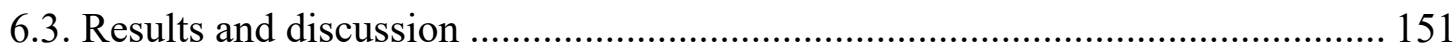

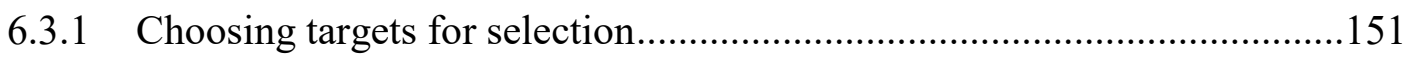

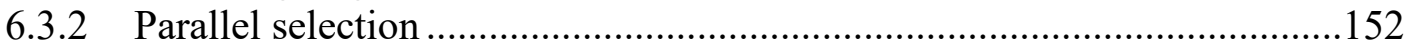




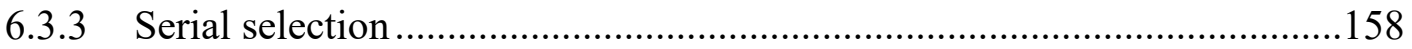

6.3.4 Characterization and sequencing of the final serial pool ...........................159

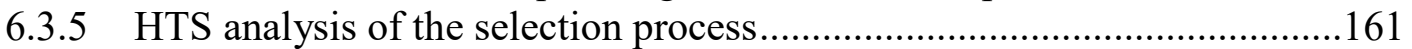

6.3.6 Characterization of the affinity and specificity of the isolated aptamer ....164

6.3.7 Evaluating the target-binding spectrum and specificity of the isolated

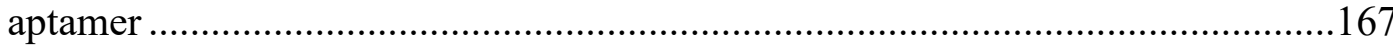

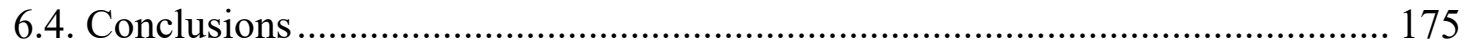

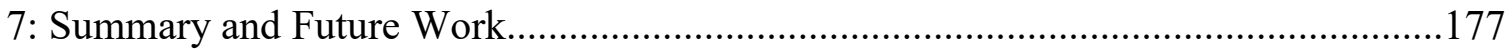

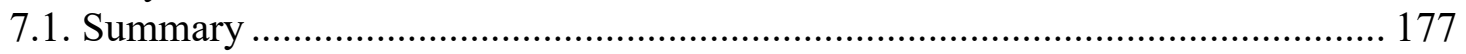

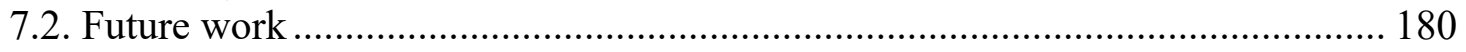

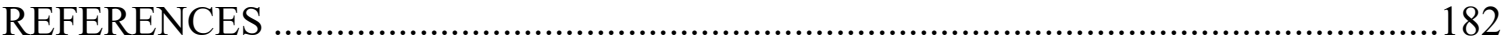

VITA …… - - 


\section{LIST OF TABLES}

TABLE

PAGE

CHAPTER 2

2-1. Comparison between aptamer and antibody in sensor development 10

\section{CHAPTER 3}

3-1. Sequence ID and DNA sequences used in Chapter 3

\section{CHAPTER 4}

4-1. The sequences of the oligonucleotide strands employed in Chapter 4 78

4-2. Comparison of assays for the visual detection of cocaine

\section{CHAPTER 5}

5-1. The sequences of DNA oligonucleotides employed in Chapter 5

\section{CHAPTER 6}

6-1. Sequences of DNA oligonucleotides employed in Chapter 6.

6-2. Detailed information regarding the conditions for each round of SELEX.

6-3. The sequences of the N30 random domain of the oligonucleotides from the final enriched pool

6-4. Binding parameters of SCA2.1 to $\alpha$-PVP, ethylone and butylone, as characterized by fitting ITC data with a one-site or modified two-site binding model .... 163 6-5. Binding parameters of SCA2.1 to (-)-MDPV, (+)-MDPV, $( \pm)-\mathrm{MDPV}$, as characterized by fitting ITC data with a one-site (for (-)- or (+)-MDPV) or modified two-site binding model (for ( \pm )-MDPV) 


\section{LIST OF FIGURES}

FIGURE

PAGE

\section{CHAPTER 2}

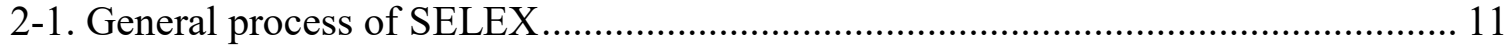

2-2. Common strategies to separate target-binding and non-binding strands.................. 13

2-3. Common SELEX strategies for control aptamer specificity ................................... 17

2-4. Different strategies to develop aptamer-based sensors........................................... 20

2-5. Working principle of exonuclease-inhibition assay for small molecule detection.... 30

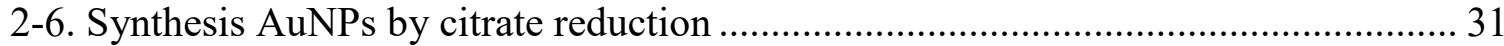

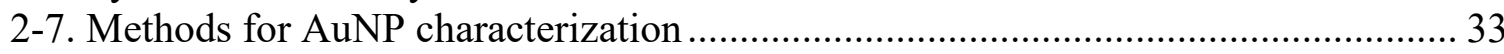

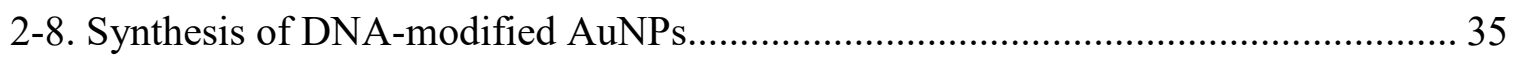

2-9. Examples of aptamer-based colorimetric assays employing AuNPs for small

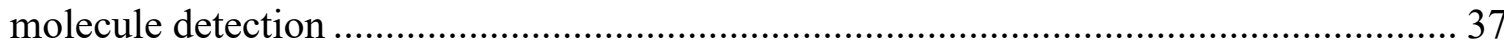

2-10. Working principle of an EATR assay for detection of DNA …………………..... 41

2-11. Chemical structure of small molecule targets......................................................... 43

\section{CHAPTER 3}

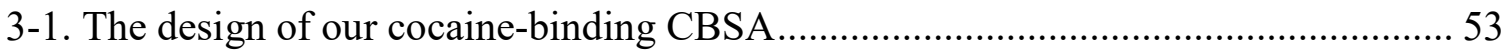

3-2. ATMND as a fluorescence reporter of target-induced CBSA assembly ................... 55

3-3. Optimization of $\mathrm{Mg}^{2+}$ and ATMND concentrations for our CBSA-based ATMND-

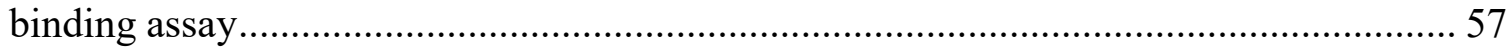

3-4. ATMND-reported calibration curve for CBSA-5325 at varying cocaine

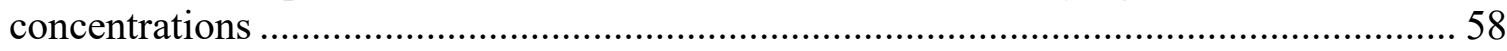

3-5. ATMND binding affinity for cocaine-assembled CBSA-5325 ……………..........59

3-6. Analyzing the dual binding domains of CBSA for target-induced assembly............ 59

$3-7 . \mathrm{K}_{\mathrm{D}}$ of $38-\mathrm{GC}$ and different $38-\mathrm{GC}$ mutants for cocaine as characterized by ITC ..... 60

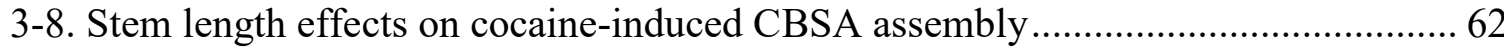

3-9. Effect of target-binding affinity $\left(\mathrm{K}_{1 / 2}\right)$ on CBSA cooperativity ............................... 63

$3-10$. Spectra of CBSA-5335-based fluorescent detection of cocaine in $1 \times$ binding

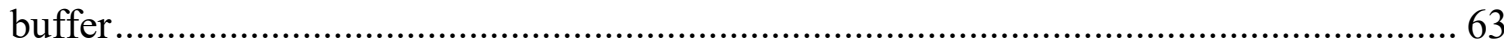

3-11. Characterization of cocaine binding affinity of CBSA-5335 and PSA using ITC .. 65

3-12. Effect of the intrinsic affinity of the PSA on CBSA target-binding affinity $\left(\mathrm{K}_{1 / 2}\right) .66$

3-13. Fluorescent detection of cocaine in saliva with our fluorophore/quencher

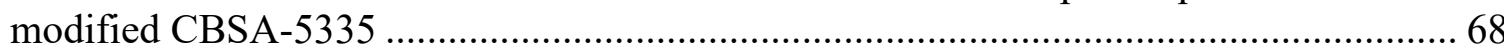

3-14. Successful detection of cocaine in saliva samples with fluorophore/quenchermodified CBSA-5335

3-15. High sensitivity and specificity of our CBSA-5335-based fluorophore/quencher assay for cocaine detection in saliva

3-16.Calibration curve of our CBSA-5335-based fluorophore/quencher assay in 1× binding buffer and $10 \%$ saliva at cocaine concentrations ranging from 0 to $10 \mu \mathrm{M}$........ 69

3-17. Bias and precision of the CBSA-5335-based fluorophore/quencher assay 


\section{CHAPTER 4}

4-1. Characterization of DIS binding affinity of DISS.1-AT using ITC …………......... 84

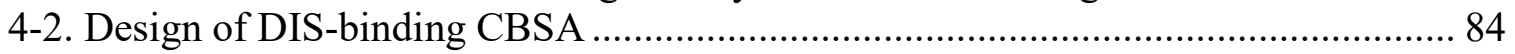

4-3. Working principle of our CBSA-based, EATR-amplified fluorescence assay ......... 86

4-4. Characterization of target-induced assembly of DIS-CBSA-4536, DIS-SA-36 and DIS-SA-536 using a fluorophore-quencher assay ..................................................... 87

4-5. Time-course of Exo III-mediated cleavage of DIS-CBSA-4536 ………................. 88

4-6. Digestion time-course of DIS-CBSA-4536 with excess DIS-SF ............................ 89

4-7. CBSA-based EATR-amplified fluorescence assay for sensitive detection of DIS ... 90

4-8. Demonstration of EATR-amplified signal in a fluorophore-quencher assay ............ 90

4-9. CBSA-based EATR-amplified fluorescence assay for sensitive detection of DIS in

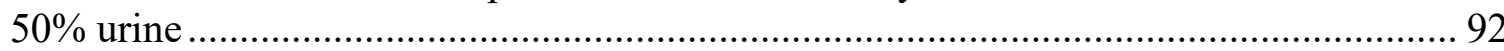

4-10. A colorimetric CBSA-based EATR-amplified assay for naked-eye detection of

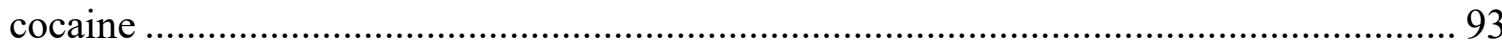

4-11. Cocaine-binding CBSA with a poly(A)s-protected long fragment and different

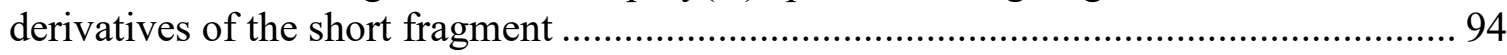

4-12. Time-course of CBSA digestion with COC-SF-5'A ……………........................... 95

4-13. Time-course of CBSA digestion with COC-SF-3'A ............................................... 96

4-14. Time-course of CBSA digestion with COC-SF-T.................................................. 96

4-15. Time-course of Exo III digestion of cocaine-binding CBSA .................................. 96

4-16. Characterizing surface coverage of SH-COC-SF-1X-modified AuNPs treated

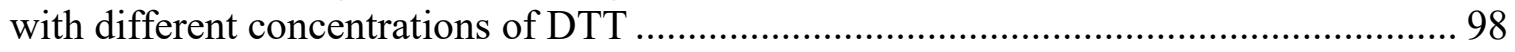

4-17. Effect of SH-COC-SF-1X surface coverage ……….......................................... 99

4-18. Alternative version of our colorimetric cocaine sensor using SH-COC-SF-2X-

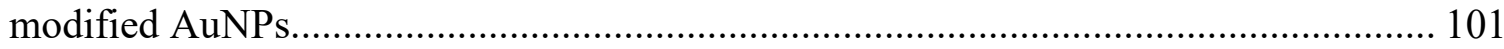

4-19. Surface coverage of SH-COC-SF-2X-modified AuNPs treated with different

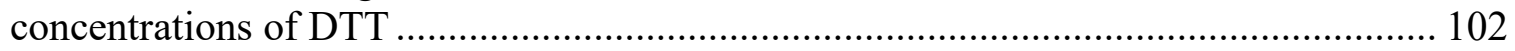

4-20. Effect of SH-COC-SF-2X surface coverage ………........................................ 102

4-21. Comparison of different SF in the CBSA-based EATR-amplified colorimetric

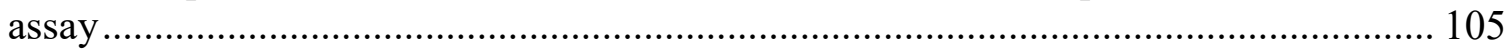

4-22. CBSA assay for cocaine detection with SH-COC-SF-2X-conjugated AuNPs ..... 105 4-23. Specificity of the EATR-amplified CBSA-based colorimetric assay for $50 \mu \mathrm{M}$

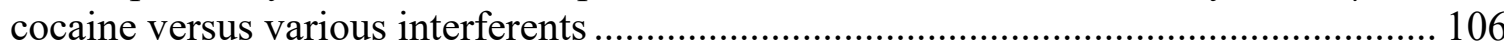

\section{CHAPTER 5}

5-1. Cy7 binding to a TWJ-containing structured DNA pool in a sequenceindependent manner.

5-2. Generality of target-induced displacement of Cy7 from aptamers with a TWJ-

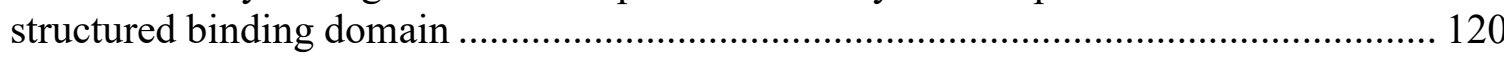

5-3. Characterization of Cy7 binding to the isolated stems from the TWJ pool ............ 121

5-4. Characterization of Cy7 binding to the TWJ pool................................................... 121 
5-5. Isolation of TWJ-structured aptamers for a Cy7-displacement small-molecule detection assay

5-6. Estimation of target affinity and specificity of the enriched pools during SELEX using a target elution assay

5-7. Sequence logo for 50 clones isolated after 10 rounds of SELEX, showing the relative frequency of every nucleotide at each position................................................ 125

5-8. Binding affinity of MDPV to MA and MA-Cy7 complex ..................................... 126

5-9. Detection of MDPV with a label-free Cy7-displacement colorimetric assay ......... 127

5-10. High cross-reactivity of MA to synthetic cathinone drugs and high specificity

against other structurally-similar or -dissimilar interfering agents ............................. 128

5-11. Cross-reactivity and specificity of our Cy7-displacement assay with $250 \mu \mathrm{M}$

MDPV, other synthetic cathinones, or structurally-similar non-cathinone compounds

and common cutting agents

5-12. Cross-reactivity of our Cy7-displacement assay with $50 \mu \mathrm{M}$ MDPV, cocaine,

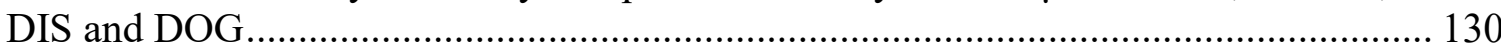

5-13. Working principle of a conventional strand-displacement fluorescence assay ..... 132

5-14. Strand-displacement fluorescence assay based on the MA aptamer ...................... 132

5-15. Affinity of MDPV to MA-L, cDNA-MA-L complex, and Cy7-MA-L complex.. 133

\section{CHAPTER 6}

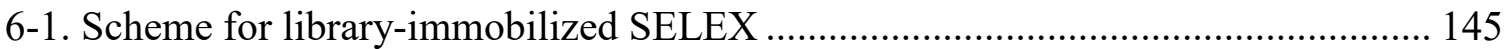

6-2. Scheme of the gel elution assay....................................................................... 147

6-3. Isolation of a class-specific aptamer using a parallel-and-serial SELEX strategy .. 152

6-4. Determination of the target-binding affinity, cross-reactivity, and specificity of

the round P5 ethylone pool via gel-elution assay ………............................................ 153

6-5. Determination of the target-binding affinity, cross-reactivity, and specificity of

the round P5 butylone pool via gel-elution assay ........................................................... 153

6-6. Determination of the target-binding affinity, cross-reactivity, and specificity of

the round P5 $\alpha$-PVP pool via gel-elution assay .......................................................... 154

6-7. Determination of the target-binding affinity, cross-reactivity, and specificity of

the round P9 $\alpha$-PVP pool via gel-elution assay

6-8. Determination of the target-binding affinity, cross-reactivity and specificity of

the round $\mathrm{S} 3$ pool via a gel elution assay.....

6-9. Characterization of the affinity and specificity of the final enriched pool via a gel-

elution assay.

6-10. Chemical structures

6-11. Secondary structure of SCA2.1 as predicted by Mfold at selection buffer ion

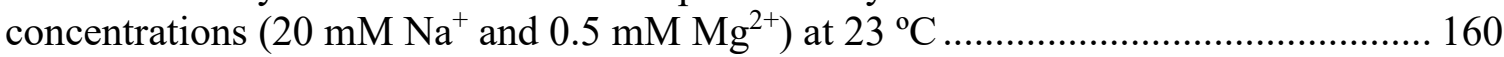

6-12. Box and whisker plots of the population distribution of sequences ...................... 162

6-13. Characterization of the target-binding affinity of SCA2.1 using ITC ................... 163

6-14. Characterization of the synthetic-cathinone-binding affinity of SCA2.1 using

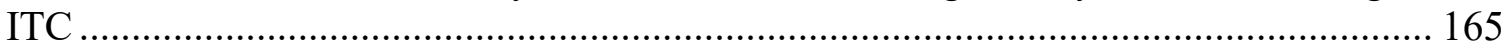

6-15. Characterization of the target-binding affinity of SCA2.1 using ITC ................... 165

6-16. Characterization of the interferent-binding affinity of SCA2.1 using ITC ........... 166 
6-17. Colorimetric detection of synthetic cathinones using a Cy7-displacement assay. 169 6-18. Determination of the binding affinity of $\mathrm{Cy} 7$ to SCA2.1 via a colorimetric assay 169 6-19. Cy7-displacement colorimetric assay for the detection of synthetic cathinones using SCA2.1

6-20. Calibration curves based on the absorbance ratio at $670 / 775 \mathrm{~nm}$ in the presence of different concentrations of $\alpha$-PVP, ethylone or butylone in buffer 170 6-21. Detection of ethylone in 50\% urine using an SCA2.1-based Cy7-displacement colorimetric assay .... 170 6-22. Detection of ethylone in 50\% saliva using the SCA2.1-based Cy7-displacement colorimetric assay 6-23. Colorimetric detection of synthetic cathinones using a Cy7-displacement assay. 172 6-24. Calibration curves and linear ranges for the SCA2.1-based Cy7-displcaement assay in $50 \%$ urine based on the absorbance ratio at $670 / 775 \mathrm{~nm}$ in the presence of different concentrations of various synthetic cathinones....

6-25. Naked-eye detection of ethylone in the concentration range of $0.4 \mu \mathrm{M}$ to $200 \mu \mathrm{M}$ 


\section{LIST OF ABBREVIATIONS}

ACRONYMS

ANOVA

AP

CBSA

cDNA

CE

CV

DLS

DRUID

E.g.

EATR

ELISA

Et al.

FET

HPLC

HTS

I.e.

ITC

LF

LOD

LSA
Analysis of variance

Apurinic/apyrimidinic

Cooperative-binding split aptamer

Complementary DNA

Capillary electrophoresis

Coefficient of variation

Dynamic light scattering

European Union's Driving Under the Influence of Drugs, Alcohol and Medicines

exempli gratia

Enzyme-assisted target recycling

Enzyme-linked immunosorbent assay

Et alia

Field-effect transistors

High performance liquid chromatography

High throughput sequencing

id est

Isothermal titration calorimetry

Long fragment

Limit of detection

Long split aptamer 
LSPR

NESA

NMR

PAGE

PCR

PSA

SELEX

SF

SSA

TEM

TWJ

\section{CHEMICALS AND MATERIALS}

3-FMC

4-FMC

4-MMC

A

$\mathrm{ACM}$

AMP

ATMND

ATP

AuNP

BSA

Adenine
Localized surface plasmon resonance

Nicking enzyme signal amplification

Nuclear magnetic resonance

Polyacrylamide gel electrophoresis

Polymerase chain reaction

Parent split aptamer

Systematic evolution of ligands by exponential enrichment

Short fragment

Short split aptamer

Transmission electron microscopy

Three-way junction

4-Fluoromethcathinone

4-Methylmethcathinone

Acetaminophen

Amphetamine

2-Amino-5,6,7-trimethyl-1,8-naphthyridine

Adenosine-5'-triphosphate

Gold nanoparticle

Bovine serine albumin 


$\begin{array}{ll}\text { BZE } & \text { Benzoylecgonine } \\ \text { C } & \text { Cytosine } \\ \text { COC } & \text { Cocaine } \\ \text { Cy5 } & \text { Diethylthiadicarbocyanine } \\ \text { Cy7 } & \text { Diethylthiatricarbocyanine } \\ \text { DHEA } & \text { Dehydroepiandrosterone } \\ \text { DIS } & \text { Dehydroepiandrosterone sulfate } \\ \text { DMSO } & \text { Dimethyl sulfoxide } \\ \text { DNA } & \text { Deoxyribonucleic acid } \\ \text { DOG } & \text { Deoxycorticosterone-21 glucoside } \\ \text { DTT } & 1,4-\text { Dithiothreitol } \\ \text { EC } & \text { Cocaethylene } \\ \text { EDTA } & \text { Ethylenediaminetetraacetic acid } \\ \text { EPH } & \text { Ephedrine } \\ \text { Exo I } & \text { Exonuclease I } \\ \text { Exo III } & \text { Exonuclease III } \\ \text { FAM } & \text { 6-Carboxyfluorescein } \\ \text { G } & \text { Guanine } \\ \text { GO } & \text { Graphene oxide } \\ \text { LDC } & \text { Lidocaine } \\ \text { MDPBP } & 3,4-\text { Methylenedioxy- } \alpha \text {-pyrrolidinobutiophenone } \\ \text { MDPV } & \end{array}$




\begin{tabular}{|c|c|}
\hline MEG & Anhydroecgonine methyl ester \\
\hline МePBP & 4-Methyl- $\alpha$-pyrrolidinobutiophenone \\
\hline METH & Methamphetamine \\
\hline MPHP & 4'-Methyl- $\alpha$-pyrrolidinohexiophenone \\
\hline $\mathrm{N}$ & Random nucleobase \\
\hline NIC & Nicotine \\
\hline PRC & Procaine \\
\hline PSE & Pseudoephedrine \\
\hline RNA & Ribonucleic acid \\
\hline $\mathrm{T}$ & Thymine \\
\hline TBE & Tris/Borate/EDTA \\
\hline Tris & Tris(hydroxymethyl)aminomethane \\
\hline $\mathrm{U}$ & Uracil \\
\hline$\alpha-P V P$ & Alpha-pyrrolidinopentiophenone \\
\hline \multicolumn{2}{|c|}{ UNITS AND PROPERTIES } \\
\hline$\$$ & US dollar \\
\hline$\%$ & Percent \\
\hline${ }^{\circ} \mathrm{C}$ & Degrees Celsius \\
\hline$\Delta \mathrm{H}$ & Enthalpy change \\
\hline$\Delta \mathrm{S}$ & Entropy change \\
\hline$\mu \mathrm{L}$ & Microliter \\
\hline$\mu \mathrm{M}$ & Micromolar \\
\hline
\end{tabular}




\begin{tabular}{|c|c|}
\hline cal & Calorie \\
\hline $\mathrm{Da}$ & Dalton \\
\hline $\mathrm{K}_{1 / 2}$ & $\begin{array}{l}\text { Ligand concentration producing half occupation in Hill } \\
\text { equation }\end{array}$ \\
\hline$K_{D}$ & Dissociation constant \\
\hline $\mathrm{mg}$ & Milligram \\
\hline $\min$ & Minute \\
\hline $\mathrm{mL}$ & Milliliter \\
\hline $\mathrm{mM}$ & Millimolar \\
\hline $\mathrm{N}$ & binding stoichiometries \\
\hline $\mathrm{n}_{\mathrm{H}}$ & Hill coefficient \\
\hline $\mathrm{nm}$ & Nanometer \\
\hline $\mathrm{nM}$ & Nanomolar \\
\hline $\mathrm{pM}$ & Picomolar \\
\hline $\mathrm{rcf}$ & Relative centrifugal force \\
\hline $\mathrm{U}$ & Activity unit \\
\hline $\mathrm{w} / \mathrm{v}$ & Weight/volume \\
\hline$\varepsilon$ & Extinction coefficient \\
\hline$\lambda$ & Wavelength \\
\hline
\end{tabular}




\section{CHAPTER 1: Introduction}

\subsection{Overview}

Aptamers are single-stranded oligonucleotide-based bioaffinity elements isolated in vitro via a technology termed "systematic evolution of ligands by exponential enrichment" (SELEX). Since 1990, hundreds of aptamers have been isolated for various targets including proteins, small molecules, metal ions, and whole cells. More recently, the analytical power of aptamers in biosensors has been demonstrated, particularly for the detection of small-molecule targets such as illicit drugs, environmental toxins, antibiotics, and biomarkers. Compared with antibodies, the dominant class of biorecognition elements used in current biosensors, aptamers have several remarkable advantages. First, unlike antibodies, which are isolated in vivo via immunization of animals, the aptamer isolation process is entirely in vitro. Thus, aptamers can easily be isolated even for nonimmunogenic and toxic small-molecule targets. Moreover, aptamer properties such as target-binding affinity and specificity can be controlled during the SELEX process by adopting different selection strategies and manipulating the selection conditions. In contrast, the in vivo nature of antibody generation forbids such control over antibody binding properties. Second, isolated aptamers can be chemically synthesized at low cost and with minimum batch-to-batch variation, overcoming two major drawbacks of antibody production. Third, compared to peptide-based antibodies, which suffer from irreversible degradation and denaturation under harsh conditions (e.g., high temperature), aptamers (particularly DNA-based aptamers) have higher chemical and thermal stability,

conferring a much longer shelf life than antibodies—even at room temperature. Finally, post-SELEX sequence engineering and chemical modification of aptamers can be 
routinely performed via chemical synthesis, which allows facile incorporation of different sensing functionalities into aptamers for target detection. However, engineering of antibodies is typically challenging and often limits their sensing utility. As a result, numerous aptamers have been isolated for small molecules and adopted into various sensing platforms for diverse applications such as drug detection, environmental monitoring, food safety, and clinical diagnostic.

Newly-isolated aptamers usually have a fully-folded structure. Although they may possess high target-binding affinity, these aptamers generally lack signal-reporting functionality for target detection. A few assays based on fully-folded aptamers have been reported; however, they are usually limited to specific aptamers or require specific instruments, and there is currently no generalizable method to isolate fully-folded aptamers with innate signal-reporting functionality. To incorporate such functionality, aptamers are routinely cleaved to derive structure-switching aptamers or split aptamers. These engineered aptamers have lower thermal stability compared with their fully-folded parent aptamers, and predominately display a single-stranded conformation with disrupted target binding domains in the absence of target. Nevertheless, these aptamer fragments maintain target-binding affinity and can fold or assemble into a duplexed structure similar to their parent aptamer upon addition of target molecules. When modified with a fluorescent, electrochemical, or enzymatic label, such target-induced conformational changes can be readily transduced into a measurable signal for target detection. Unfortunately, the low thermal stability of structure-switching or split aptamers inevitably leads to reduced target-binding affinity compared with their parent aptamers, which results in poor assay sensitivity. This is particularly troublesome for small- 
molecule binding aptamers, which often have relatively low target-binding affinity, with dissociation constants $\left(\mathrm{K}_{\mathrm{D}}\right)$ ranging from high nanomolar to low micromolar. Therefore, general approaches for the development of highly sensitive aptamer-based sensors for small-molecule targets would be highly valuable.

\subsection{Motivation and goals}

The primary research objective in this dissertation was to develop new general strategies to isolate and engineer functionalized aptamers for sensitive detection of smallmolecule targets. To achieve this goal, we implemented four innovative approaches: 1) engineering new aptamer constructs that have both high target-binding affinity and sensitive target response, 2) developing an efficient signal amplification strategy to increase the sensitivity of current aptamer-based sensors, 3) developing a generalizable sensing platform utilizing fully-folded aptamers, and 4) establishing a novel selection strategy to isolate class-specific small-molecule-binding aptamers.

First, we developed a general aptamer engineering strategy in which we incorporated cooperative-binding functionality into split aptamers to enhance their target response for more sensitive small-molecule detection (see Chapter 3). Conventional split aptamers containing one target-binding domain typically possess low target affinity and weak target response, resulting in low sensitivity when employed in biosensors. To overcome this challenge, we engineered novel cooperative-binding split aptamer (CBSA) constructs, which incorporate two cooperative binding domains such that target binding at one domain greatly increases the affinity of the second domain. The cooperativebinding functionality of CBSAs allows them to achieve higher target-binding affinity and more sensitive target-induced aptamer assembly compared to their parent split aptamer 
with a single binding domain. This means that rapid ultrasensitive detection of smallmolecule targets can be readily achieved using a CBSA modified with a fluorophorequencher pair. Importantly, the strategy of CBSA engineering is straightforward and can be generalized to any aptamer with a three-way junction (TWJ) secondary structure. Given the fact that many TWJ-structured aptamers have been isolated for various small molecules, CBSA-based sensors can be readily developed for the detection of various small-molecule targets, such as illicit drugs, biomarkers, toxins, and therapeutics.

Upon successful engineering of CBSAs, we further developed a generalizable enzyme-assisted target recycling (EATR) approach to enhance the analytical power of CBSAs (see Chapter 4). Specifically, we introduced a C3 spacer abasic site between the two target-binding domains of the CBSA. When the CBSA assembles upon target binding, the C3 spacer in the CBSA duplex offers a substrate that can be efficiently cleaved by Exo III. This cleavage event releases target molecules into solution, which then mediates more cycles of target-induced CBSA assembly and enzyme cleavage until all CBSAs are degraded. In the absence of target, the CBSA fragments remain separated, forming no duplexed abasic site, and are thus spared from Exo III digestion. Our EATR strategy serves two purposes. First, since a small amount of target can result in cleavage of far large numbers of CBSAs, EATR can be used as an efficient signal amplification mechanism to increase the sensitivity of CBSA-based sensors. Alternatively, EATRmediated aggregation of CBSA-modified gold nanoparticles (AuNPs) can be employed to develop an instrument-free colorimetric assay. Given the generality of our CBSA engineering strategy and the versatility of the EATR approach, such assays should provide a valuable means for small-molecule detection in various fields. 
We have further developed a general strategy to isolate fully-folded aptamers with intrinsic dye-displacement functionality for sensitive colorimetric detection of small molecules (see Chapter 5). Previous studies have shown that dye-displacement assays have several exceptional advantages, being rapid, highly sensitive, and label-free. However, the implementation of such methods has been limited, since finding a signalreporting small-molecule dye for a specific aptamer is difficult. To solve this problem, we first determined that the dye diethylthiatricarbocyanine $(\mathrm{Cy} 7)$ can stack into DNA TWJs in a sequence-independent fashion, producing a dramatic color change. We then designed a SELEX strategy utilizing a structured library containing a TWJ target-binding domain to isolate small-molecule binding aptamers. Aptamers isolated through this library intrinsically bind to $\mathrm{Cy} 7$, and thus can be directly incorporated into a Cy7displacement assay for rapid, sensitive, and colorimetric target detection. Given the high generality of our SELEX strategy and the excellent performance of the dye-displacement platform, we foresee that our approaches can be used for sensitive colorimetric detection of various small-molecule targets in diverse applications.

We finally developed a novel 'parallel-and-serial' selection strategy for isolating class-specific oligonucleotide-based aptamers in vitro (see Chapter 6). This strategy first entails parallel selection to selectively enrich cross-reactive binding sequences, followed by serial selection that enriches aptamers binding to a designated target family. As a demonstration, we isolated a class-specific DNA aptamer against a family of designer drugs known as synthetic cathinones. The aptamer binds to 12 diverse synthetic cathinones with nanomolar affinity and does not respond to 11 structurally similar nontarget compounds, some of which differ from the cathinone targets by a single atom. This 
is the first account of an aptamer exhibiting a combination of broad target crossreactivity, high affinity and remarkable specificity. Leveraging the qualities of this aptamer, instantaneous colorimetric detection of synthetic cathinones at nanomolar concentrations in biological samples was achieved. Our findings significantly expand the binding capabilities of aptamers as class-specific bioreceptors and further demonstrate the power of rationally designed selection strategies for isolating customized aptamers with desired binding profiles. We believe that our aptamer isolation approach can be broadly applied to isolate class-specific aptamers for various small molecule families.

\subsection{Scope of the dissertation}

Chapter 2 provides a general literature review of the historical background of aptamers, SELEX, aptamer-based sensors for small molecule detection, AuNPs and their use in aptamer-based sensors, EATR, and the small-molecule targets used in the work. Chapter 3 details a strategy for engineering highly target-responsive CBSA for sensitive detection of small molecules and demonstrates the application of CBSAs for rapid fluorescent detection of cocaine in saliva samples. Chapter 4 further describes a generalizable EATR approach both as a signal amplification method to increase the sensitivity of the CBSA-based fluorescence assay and a signal reporting strategy for colorimetric detection of small molecules. Chapter 5 describes a new SELEX strategy to isolate fully-folded aptamers with intrinsic dye-displacement functionality for rapid, sensitive, and colorimetric detection of any small-molecule targets. Chapter 6 describes a generally applicable selection strategy to isolate class-specific aptamers as well as a rapid sensitive assay for visual detection of a family of synthetic cathinones. Chapter 7 provides a summary of the dissertation and proposes future research directions. 
CHAPTER 2: Background and Literature Review

\subsection{Aptamers and their use as affinity reagents for small-molecule targets}

Aptamers are single-stranded DNA- and RNA-based bioaffinity elements isolated in vitro from randomized libraries via a procedure called SELEX. ${ }^{1}$ The term 'aptamer' was derived from the Greek word aptus, or "to fit". ${ }^{2}$ These oligonucleotides typically are short in length, ranging from 20 - to 80 -nt, but often possess target-binding specificity that rivals antibodies. ${ }^{3}$ Similar to antibodies, ligand recognition by aptamers is based on noncovalent interactions such as electrostatic interactions, hydrogen bonds, pi-pi interactions, and van der Waals forces. ${ }^{4}$ The first aptamers targeting protein ${ }^{1}$ and small-molecule ${ }^{2}$ targets were isolated in 1990. Since then, hundreds of aptamers have been isolated for a great diversity of targets including metal ions, small molecules, proteins, and even whole cells. $^{5}$

Inspired by naturally-occurring functional RNAs such as ribosomes and ribozymes, the first aptamers were isolated from RNA-based libraries. However, RNA-based aptamers have low chemical stability due to the presence of a reactive 2' hydroxyl group on their ribose backbone, and are prone to chemical and enzymatic hydrolysis. ${ }^{6}$ Although substitution of the 2' hydroxyl group with a fluorine or amine can increase the stability of the aptamer, these chemical modifications significantly increase the cost of aptamer production and may also reduce the binding affinity of the aptamer. ${ }^{7}$ In contrast, the deoxyribose backbone of DNA does not have the 2' hydroxyl group, and thus has much higher chemical stability and resistance to hydrolysis. In 1992, the first DNA-based aptamer was isolated against thrombin, with a $\mathrm{K}_{\mathrm{D}}$ of $25 \mathrm{nM} .^{8}$ Since then, many DNA aptamers have been isolated with comparable performance to RNA aptamers. At present, 
more than $50 \%$ of reported aptamers are composed of DNA due to their high stability, ease of handling, and commercial availability. ${ }^{5}$

Recently, a number of groups have intensively explored the utility of aptamers as biorecognition elements for small-molecule detection. Sensitive and accurate detection of small-molecule targets is critical for diverse fields including law enforcement, environmental monitoring, clinical diagnostics, and medical treatment. ${ }^{9-11}$ Instrumental methods such as gas chromatography and mass spectroscopy have very high sensitivity and specificity, but these methods are expensive, time-consuming, and rely on cumbersome instruments and trained personnel. ${ }^{12}$ Moreover, lengthy sample preparation steps are required when dealing with complex sample matrices such as biofluids. ${ }^{12}$ These disadvantages greatly limit the utility of these methods for rapid and on-site applications. ${ }^{12,13}$ Biosensors employing specific binding between targets and biorecognition elements offer a powerful alternative for the rapid detection of small molecules. For example, antibodies can bind to their targets with nanomolar or lower $K_{D S}$ with very high

specificity, allowing for highly sensitive detection of targets even in complex samples. ${ }^{14}$ Antibodies can also be coated onto paper substrates to fabricate low-cost lateral-flow devices, ${ }^{14}$ and on-site biosensing currently relies heavily on such immunoassays.

\subsubsection{Advantages of aptamers as biorecognition elements for small molecule detection}

Aptamers, as newly emerged biorecognition elements, offer several exceptional advantages for sensors compared with antibodies (Table 2-1). ${ }^{15,16}$ The generation of antibodies relies on antigen-induced immune response, but small molecules have low molecular weight $(<1,000 \mathrm{Da})$ and are typically poorly immunogenic. Therefore, small- 
molecule targets generally need to be modified with a linker and conjugated to a carrier protein prior to immunization. ${ }^{17}$ Such a process can be challenging due to the limited number of functional groups on the target for conjugation chemistry. In contrast, the in vitro nature of SELEX technology means that aptamers can be directly isolated for virtually any small molecule. Furthermore, the whole process of antibody generation, which includes target preparation, immunization of animals, antibody characterization, and antibody purification, is lengthy and labor intensive, and can take more than six months. ${ }^{18}$ In contrast, aptamer isolation is typically fast and only takes several weeks. This is critical for rapidly evolving small-molecular targets such as designer drugs, where the lengthy antibody-development process has become a significant barrier for forensic laboratories in keeping up with the diversity of new drugs on the streets. ${ }^{18,19}$ The process of antibody production is not only expensive, but also leads to large batch-to-batch variation. ${ }^{5,14}$ Moreover, it is difficult to precisely engineer and chemically modify antibodies for signal reporting, and such modifications can often significantly reduce target-binding affinity of the antibodies. ${ }^{20}$ On the other hand, aptamers can be chemically synthesized with low production cost and minimal batch-to-batch variation, and sequence engineering of aptamers is also straightforward, enabling introduction of diverse sensing functionalities. Indeed, various chemistries are commercially available to modify aptamers with different fluorescent, ${ }^{21}$ electrochemical, ${ }^{22}$ or enzymatic ${ }^{23}$ tags for signal reporting purposes.

Depending on the application, it may be crucial for biosensors to either be highly specific to a single target (to prevent false-positive results) or broadly cross-reactive to a class of structurally-related targets (to detect multiple targets using one bioreceptor). 
Because antibody generation is an in vivo process, neither affinity nor specificity of the antibodies can be controlled. ${ }^{17}$ As a result, current immunoassays for small-molecule detection routinely suffer from false-positives generated by non-specific antibody binding. Meanwhile, immunoassays are usually not able to detect a class of structurallyrelated targets since a minor modification on a target's core structure may completely impair binding. ${ }^{24}$ On the other hand, various selection strategies such as "counterSELEX" 25 or "toggle-SELEX" 26 can be employed to customize the binding-profile of isolated aptamers and generate highly specific or class-specific aptamers.

Table 2-1. Comparison between aptamer and antibody in sensor development.

\begin{tabular}{ccc}
\hline Features & Antibody & Aptamer \\
\hline Target limitation & $\begin{array}{c}\text { Limited for small molecules } \\
\text { and toxic compounds } \\
\text { Production period }\end{array}$ & No limitation \\
Production cost & High & Weeks \\
Batch-to-batch variation & High & Low \\
Binding profile & Uncontrollable & Low \\
Chemical stability & Low & Highly tunable \\
\hline \hline
\end{tabular}

As proteins, antibodies are thermodynamically unstable and undergo irreversible denaturation under harsh conditions, such as high temperature or high-salt condition. Therefore, immunoassays generally need to be refrigerated and usually have a shelf life of less than a year. ${ }^{15}$ In contrast, aptamers—especially those based on DNA—are more chemically and thermally stable than antibodies, and can be kept at room temperature for years without losing bioactivity. ${ }^{5}$ The stability of aptamers can be further enhanced by 
chemical modification of the ribose backbone with 2-fluorine or 2-O-methyl groups, to resist degradation from enzymes in the blood. ${ }^{5}$

\subsection{Isolation of small-molecule-binding aptamers via SELEX}

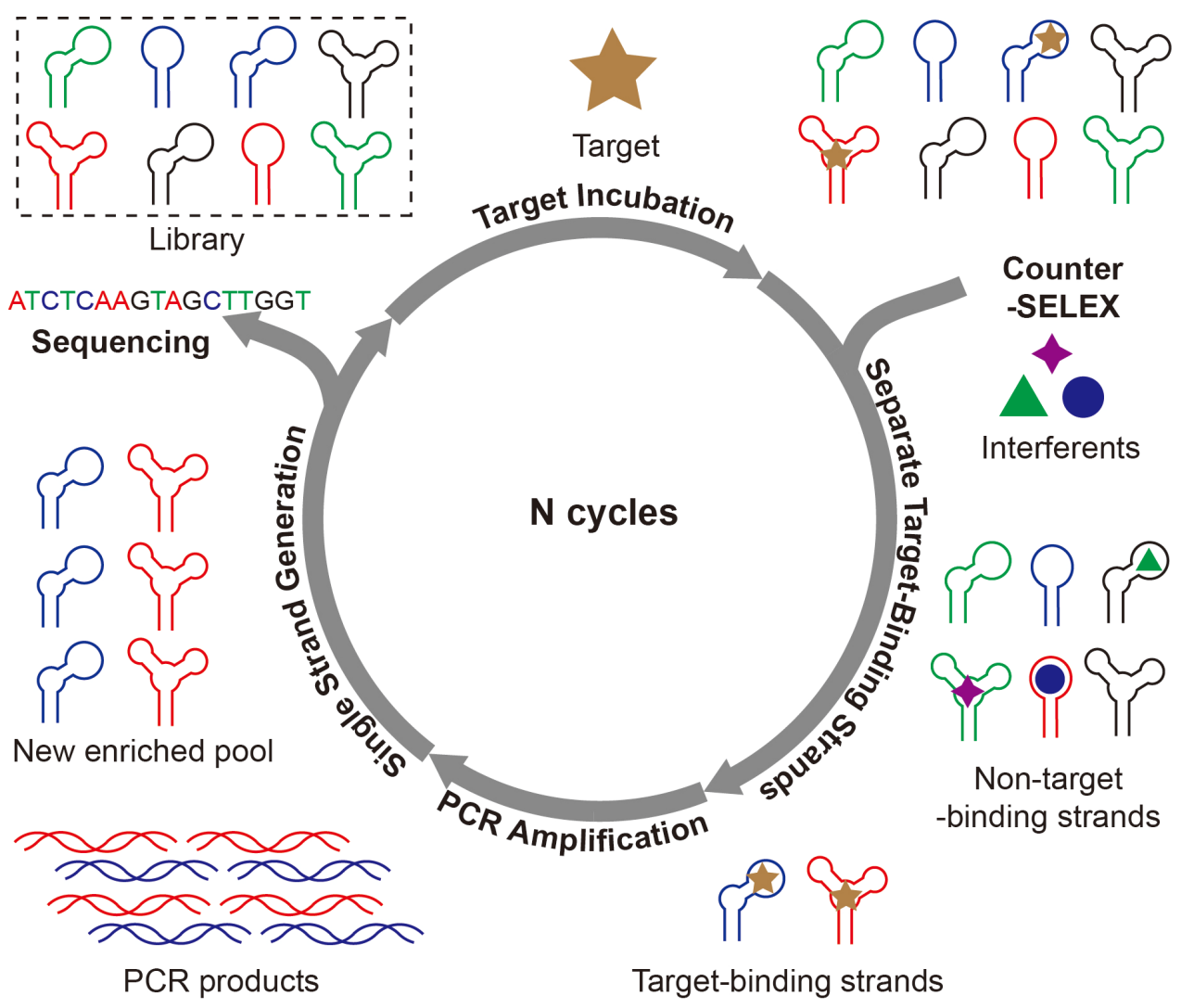

Figure 2-1. General process of SELEX.

Aptamers are isolated from randomized DNA or RNA libraries through a multi-round SELEX procedure. Each round of isolation consists of three essential steps: separation of target-binding strands, polymerase chain reaction (PCR) amplification of the targetbinding strands, and reconstruction of the new library from the isolated sequences. Strands binding to the target with higher affinity are enriched more than non-binders during each round. Therefore, after several rounds of SELEX, strands with the highest target-binding affinity will dominate the library and can be identified by sequencing the pool. Figure 2-1 shows a schematic outline of the SELEX process. 


\subsubsection{Library design}

Libraries used for aptamer isolation typically comprise a randomized sequence flanked by consensus sequences at both ends that serve as primer-binding sites. The library can be either DNA or RNA; DNA libraries can be directly synthesized, while RNA libraries are generated by in vitro transcription of the DNA library. The number of nucleotides $(\mathrm{N})$ in the random region determines the total possible sequences $\left(4^{\mathrm{N}}\right)$ in the library, as each position has four possible nucleotides (A, T, C and $\mathrm{G}$ for DNA; A, U, C, and G for RNA). The length of the random region can range from 6 to $200 \mathrm{nt}^{27}$ A long random region has more sequence diversity, which in principle allows for the formation of more complicated motifs that may benefit aptamer affinity and specificity. However, not all possible sequences in these libraries can be practically represented during SELEX. ${ }^{28}$ In contrast, short random regions form simpler binding motifs but also allow for representation of every possible sequence. Notably, one recent study showed that the length of the random region has no significant correlation with the affinity of the isolated aptamers. $^{27}$ A shorter aptamer also has advantages such as easier identification of the target-binding domain, easier sequence engineering to introduce signal-transducing functionality, and lower production costs. The most widely used small-molecule binding aptamers for sensor development, which include aptamers for cocaine, ${ }^{21}$ ATP, ${ }^{29}$ and kanamycin, ${ }^{30}$ are shorter than $40 \mathrm{nt}$. In addition to the length of the random region, one can incorporate different secondary structures such as G-quadruplexes, ${ }^{31}$ stem-loops, ${ }^{32}$ and $\mathrm{TWJS}^{33,34}$ into the library to isolate more specialized aptamers with various functionalities. For example, we have recently designed a TWJ-structured library and 
isolated an aptamer with intrinsic binding affinity to the small molecule dye Cy7 for sensitive detection MDPV using a colorimetric dye-displacement assays. ${ }^{34}$

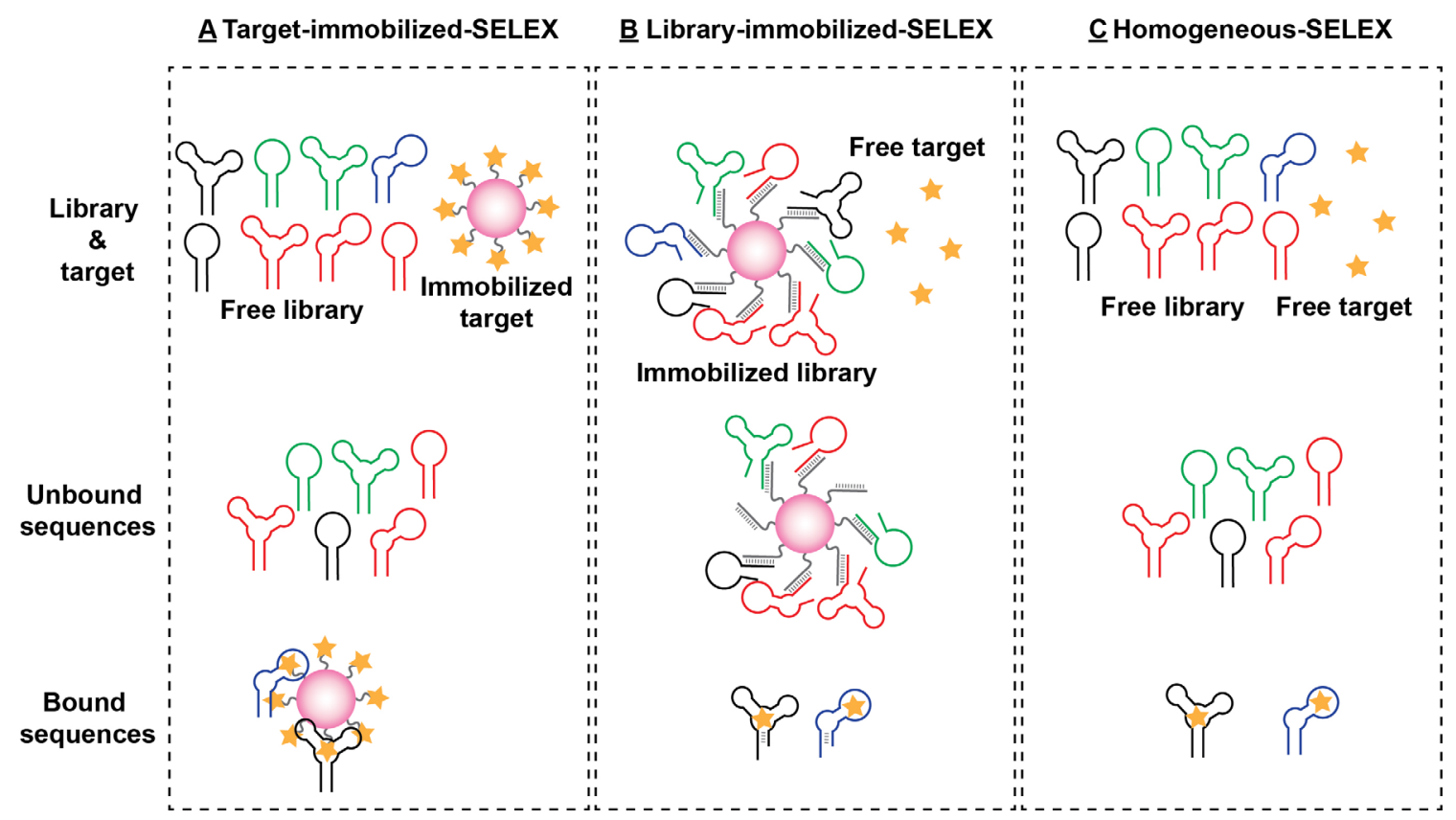

Figure 2-2. Common strategies to separate target-binding and non-binding strands. These strategies include (A) target-immobilized SELEX, (B) library-immobilized SELEX, and (C) homogeneous SELEX.

\subsubsection{Strand separation strategies for SELEX}

The success of SELEX greatly depends on the separation of target-binding strands from non-target-bound library strands. This can be achieved through either heterogeneous or homogeneous techniques. Heterogeneous (bead-based) SELEX methods have been widely utilized to isolate hundreds of aptamers for small-molecule targets such as steroids, ${ }^{33}$ nucleotides, ${ }^{29,35}$ and antibiotics, ${ }^{30,36-39}$ achieving micromolar binding affinities after $10-20$ rounds of selection. ${ }^{40}$ These methods require immobilization of either the target or library strands. For target-immobilized SELEX (Figure 2-2A), a small-molecule target is covalently conjugated on a solid-phase carrier (e.g., magnetic microbeads) via a linker. When the target-conjugated carrier is incubated with the nucleotide library, 
strands binding to the target strongly adhere to the solid phase and can be collected, while non-target-binding strands remain in the solution and can be eluted away with the buffer. Since the target is covalently conjugated onto the beads in target-immobilized methods, highly-stringent SELEX conditions can be employed to isolate aptamers within a few rounds. $^{41}$

However, these methods also have several technical challenges. ${ }^{42,43}$ Since small molecules typically have limited functional groups, surface conjugation of these targets can be challenging. Functional groups occupied by the linker may not be accessible to aptamer binding, making isolation of high-affinity aptamers difficult. In addition, both the bead surface and the linker are susceptible to non-specific recognition by library molecules, reducing separation efficiency and requiring additional negative SELEX processes to remove these non-specific binders. ${ }^{43}$ For small molecules that are not suitable for surface immobilization, library-immobilized SELEX is often used (Figure 2-2B). This method utilizes a short, bead-conjugated complementary DNA (cDNA) sequence that hybridizes to a specific region of the library strands and thereby immobilizes them onto the surface. Upon addition of the target, non-binding sequences remain on the surface, while target-binding strands undergo a conformational change that detaches them from the cDNA and releases them into solution, so that they can be collected for further enrichment. Since small-molecule targets are free in solution, nonspecific enrichment of carrier/linker binding sequences can be avoided. However, because the binding between library and capture strand is non-covalent and prone to spontaneous dissociation during target elution, ${ }^{44}$ the separation efficiency of these methods is low and requires more rounds of selection. Moreover, both target- 
immobilization and library-immobilization create steric hindrance to interactions between library and target, resulting in the isolation of aptamers with low target-binding affinity.

Alternatively, several homogeneous methods (Figure 2-2C) have been recently developed to overcome the limitations of heterogeneous SELEX, enabling higher separation efficiency and allowing for isolation of high-affinity aptamers after just a few rounds of selection. The first reported homogeneous SELEX technique utilized capillary electrophoresis separation, exploiting the differential mobility between unbound and target-bound library molecules under an external electrical field. ${ }^{45,46}$ However, this method offers limited utility for small-molecule targets, because the mobility of targetaptamer complexes is very similar to that of unbound oligonucleotides, resulting in poor separation resolution. ${ }^{47}$ Alternatively, graphene oxide has been used to remove nontarget-bound molecules during the separation step, as this material binds more strongly to single-stranded DNA than to folded, target-bound DNA structures. ${ }^{48}$ This technique has produced DNA aptamers for three pesticides and two toxins, with $\mathrm{K}_{\mathrm{D}}$ ranging from 1-100 $\mathrm{nM}$ after 5-10 rounds. ${ }^{49-51}$ However, this technique is not compatible with targets that have strong $\pi-\pi$ interaction with graphene oxide, such as doxorubicin. ${ }^{52}$ So far, there is no generally applicable homogeneous SELEX technique for isolating small-moleculebinding aptamers.

\subsubsection{Aptamer enrichment by PCR and regeneration of single-stranded libraries}

There are a few examples of aptamers isolated through a single-round selection process $^{53,54}$ or a multi-round selection process without PCR amplification. ${ }^{55}$ However, none of these methods has been successfully applied to small-molecule targets, and SELEX is almost always a multi-round process. Since target-bound strands usually 
comprise a minimal fraction of the library pool, especially in the earlier rounds, the separated target-binding strands need to be amplified by PCR to regenerate the new library for the next round of selection. For RNA-based libraries, in vitro reverse transcription is required before PCR amplification. During PCR, single-stranded library strands are converted into double-stranded products, and it is therefore necessary to remove antisense strands to regenerate the single-stranded new library for the next round of selection. Methods routinely used for library regeneration include physical separation, ${ }^{56}$ enzymatic digestion ${ }^{57,58}$, and asymmetric $\mathrm{PCR}^{59}$. In the physical separation method, a biotinylated reverse primer is used during PCR to generate a biotin-labeled antisense strand. The double-stranded PCR products are then captured by streptavidincoated microbeads and incubated with $\mathrm{NaOH}$, denaturing the double-stranded DNA and releasing the sense strands into solution. These strands are then collected and used as a new single-stranded library. Alternatively, modified primers can be used to enable enzymatic removal of antisense strands from the PCR products. For example, if PCR amplification is performed using a 5'-phosphorothioate-labeled forward primer, the antisense strands can be digested using T7 exonuclease, while the sense strands are protected by the $5^{\prime}$-phosphorothioate label and remain intact. ${ }^{57}$ Similarly, if PCR amplification is performed using a 5'-phosphorylated reverse primer, lambda exonuclease can be used to specifically remove the phosphorylated antisense strands while sparing the non-modified sense strands. ${ }^{58}$ As an alternative, asymmetric PCR allows for direct generation of single-stranded libraries by adding an excess of forward primer relative to the reverse primer. During PCR, the reverse primer is rapidly depleted so that ultimately, only the sense strand can be effectively amplified with the forward primer, producing 
single-stranded products. ${ }^{59}$ These different methods for library regeneration have been found to vary moderately in terms of cost and efficacy, ${ }^{60}$ and our work has generally employed physical separation with streptavidin-coated agarose resin. ${ }^{34,61}$

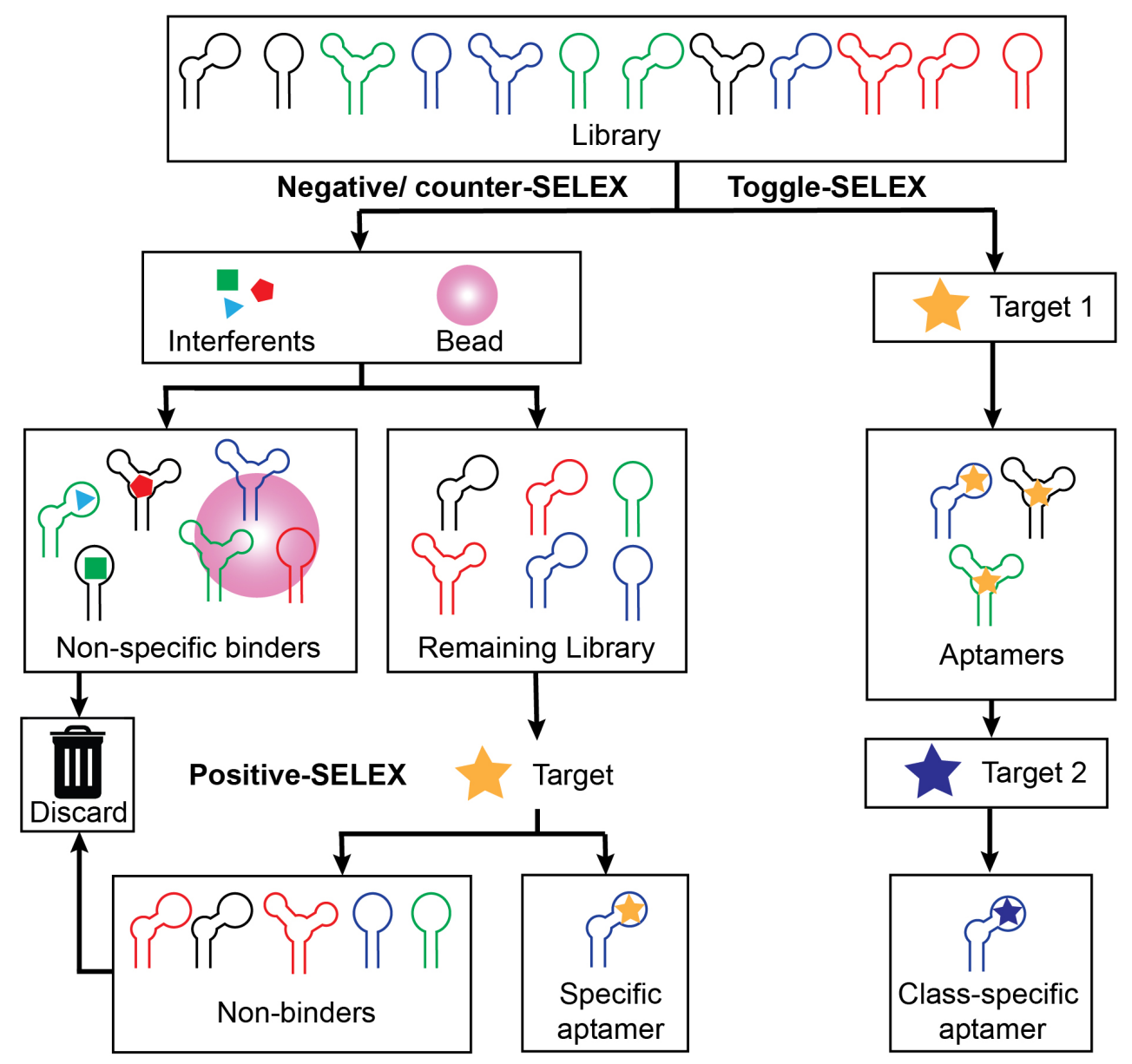

Figure 2-3. Common SELEX strategies for control aptamer specificity.

\subsubsection{Common selection strategies for control affinity and specificity of aptamers}

One of the major advantages of aptamers compared with antibodies is that their affinity and specificity can be fine-tuned during SELEX by using different selection strategies. Control of aptamer affinity is usually achieved by manipulating SELEX stringency. Under low-stringency conditions, even aptamers with low target-binding affinity can be retained in the enriched pool. However, under highly stringent conditions, 
only aptamers with the highest target affinity can survive. ${ }^{62-64}$ Common ways of modulating selection stringency include altering target concentration, target incubation time, temperature, and buffer ionic strength. ${ }^{27,62,63}$ High selection stringency results in higher aptamer affinity. However, high stringency also increases the risk of losing targetbinding sequences, especially during earlier rounds where the copy number of each sequence is low. Therefore, SELEX is usually performed with relatively lower stringency in earlier rounds to retain all possible target-binding sequences. The stringency is then gradually increased in later rounds to enrich high-affinity aptamers ${ }^{65}$ Some mathematical models have been established to guide selection stringency during the SELEX process, ${ }^{62-64}$ but in most cases the change in stringency between rounds is highly empirical. ${ }^{61}$

Some selection strategies have also been developed to control the binding specificity of the aptamers. One disadvantage of target-immobilized SELEX is the evolution of bead-binding aptamers. Negative-SELEX strategies can overcome this problem (Figure $2-3$, left) ${ }^{66}$ Here, the library is first incubated with non-modified beads, so that beadbinding strands are adsorbed onto the beads. These beads are then removed, and the remaining library strands are then incubated with target-immobilized beads for regular SELEX. A similar strategy, termed counter-SELEX, was developed to prevent isolated aptamers from binding to possible interferents (Figure 2-3, left). In 1994, using a very stringent counter-SELEX regime, Polisky et al. isolated a theophylline-binding aptamer that has a 10,000-fold higher affinity for this target compared to caffeine, which only differs from the target by a methyl group. ${ }^{25}$ After incubating theophylline-immobilized beads with the library, they used a high concentration of caffeine to elute and thereby 
eliminate library strands recognizing this molecule. Multiple rounds of counter-SELEX can be used to further fine-tune the specificity of the resulting aptamers.

The specificity of aptamers can also be tailored to recognize a whole family of targets sharing the same core structure. Such 'class-specific' aptamers can be used in many sensing applications, including detection of illicit drugs and their metabolites for forensic investigations, antibiotics for food safety, or pesticides for environmental monitoring. ${ }^{67-69}$ In order to generate class-specific aptamers, Sullenger et al. developed a toggle-SELEX strategy (Figure 2-3, right) in 2001, yielding an aptamer that cross-reacted to both human and porcine thrombin. ${ }^{26}$ The initial library was incubated with a mixture of both targets to enrich all potential aptamers, after which human and porcine thrombin were used as alternating selection targets every round. Consequently, only library strands that bind to epitopes present on both targets can be enriched in every round, while aptamers specific to one species were removed. The final aptamer bound to human and porcine thrombin with a $\mathrm{K}_{\mathrm{D}}$ of 2.8 and $0.1 \mathrm{nM}$, respectively. In comparison, an aptamer isolated using only porcine thrombin demonstrated $>10,000$-fold higher specificity for this protein relative to human thrombin. ${ }^{26}$ Toggle-SELEX has been employed to isolate several class-specific aptamers, mostly for protein and cell targets. ${ }^{70-72}$ In some cases, toggle-SELEX was also performed with more than two targets to achieve broader binding spectra. ${ }^{71,72}$ A similar toggle strategy can also be applied to isolate class-specific aptamers for small-molecule targets. ${ }^{36,39,73}$ However, this is more challenging than for protein and cell targets due to lack of epitopes on small-molecule targets. For example, one study used toggle SELEX with four different target pairs to isolate class-specific aminoglycoside-binding aptamers. However, only one out of the 80 aptamers demonstrated cross-reactivity to all eight 
aminoglycoside targets. ${ }^{36}$ To overcome such limitations, we have recently developed new parallel-and-serial selection strategies to increase target cross-reactivity and the success rate of class-specific aptamer isolation. ${ }^{74}$ As a demonstration, we have isolated a classspecific aptamer that binds to more than 12 synthetic cathinones sharing the same corestructure with nanomolar affinity.

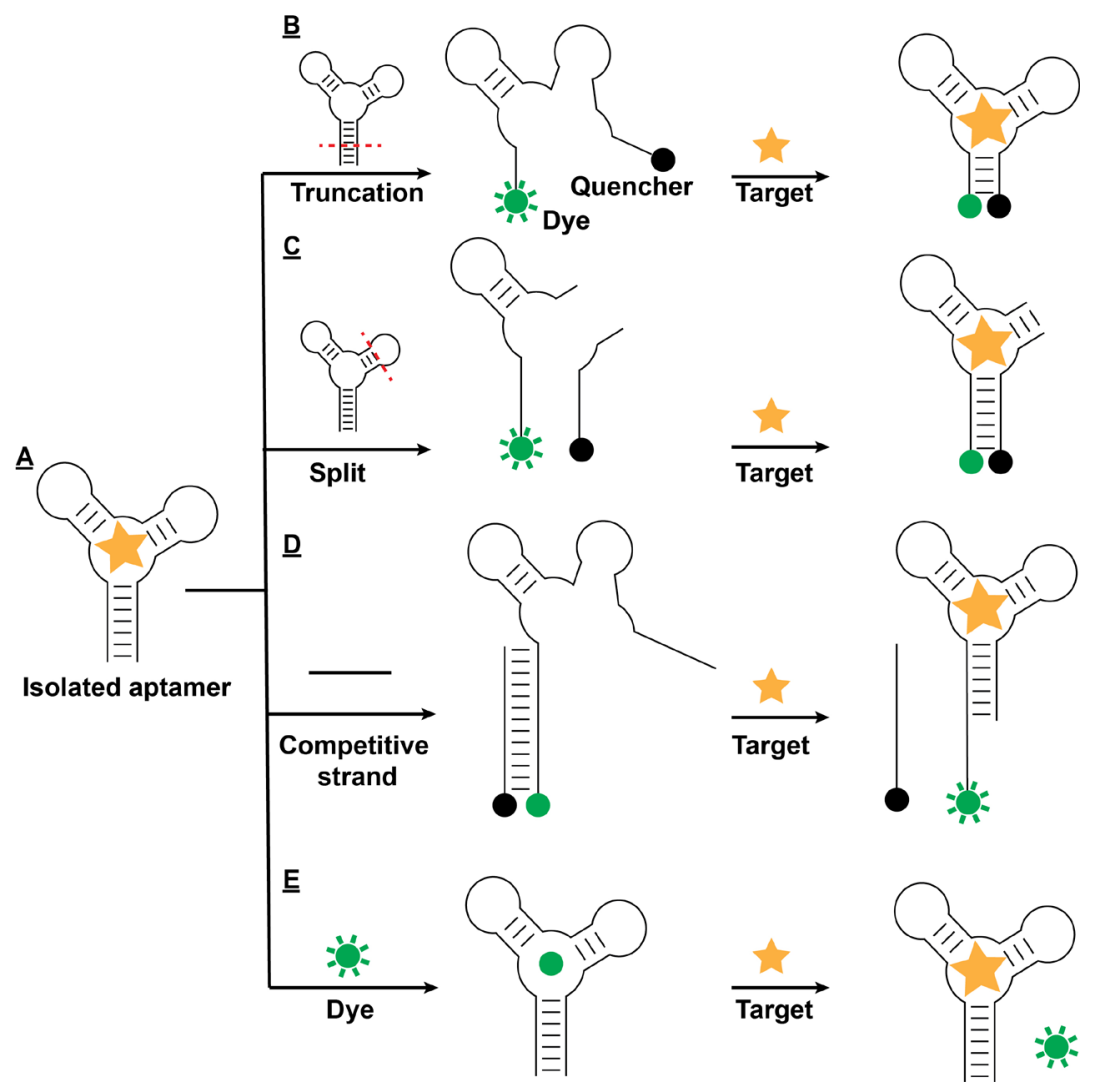

Figure 2-4. Different strategies to develop aptamer-based sensors. Sensors can be developed from (A) a fully folded aptamer via (B) aptamer truncation, (C) splitting, (D) introducing a competitive strand, or (E), a dye binding to target-binding domain of the aptamer.

\subsection{Aptamer-based sensors for small molecule detection}

As target affinity is typically the only selection pressure applied during SELEX, isolated aptamers usually lack innate signal transducing functionality and cannot be directly used for biosensing. Many strategies have been developed to introduce different signal transducing functionalities into aptamers to achieve optical, electrochemical, or 
electrical detection of small-molecule targets (Figure 2-4) ${ }^{75}$ Most aptamer-based sensors for small-molecule detection rely on a conformational change of the aptamer upon target binding - functionality that are typically be introduced into the aptamer via a multi-stage process of sequence analysis, truncation and chemical modification. ${ }^{75-77}$ Nevertheless, several sensor platforms are available that employ fully-folded aptamers without requiring target-induced conformational change.

\subsubsection{Sensors based on target-induced conformational changes}

Small-molecule-binding aptamers are usually fully folded upon isolation, with stable secondary structures (Figure 2-4A), such that target binding does not significantly alter their structure. The functionality of binding-induced conformational change is therefore introduced into the aptamer via post-SELEX. An aptamer comprises a target-binding domain and several scaffold regions. Small-molecule-binding aptamers usually have small target-binding domains, since the small surface area of the target can interact with a limited number of nucleotides simultaneously. The nucleotides in the scaffold regions do not directly interact with the target, but help to stabilize the structure of the aptamer and thereby ensure proper formation of the target-binding domain. When scaffold regions are disrupted, the aptamer will lose its secondary structure and become single-stranded. However, the disrupted sequences may retain their capacity for target recognition and to reassemble into the original secondary structure in the presence of the target. This targetinduced aptamer conformational change can then be employed for signal transduction. Aptamer truncation, splitting, and strand-displacement are the three general strategies to introduce target-induced conformational change functionality. 


\subsubsection{Sensors based on truncated structure-switching aptamer}

The most intuitive way of introducing structure-switching functionality is to truncate the aptamer (Figure 2-4B). Aptamers usually have double-stranded stems contributing to the scaffold region. Shortening these stems reduces the melting temperature of the aptamer, shifting their equilibrium to favor a single-stranded structure that can still be refolded upon target binding. For example, the well-characterized cocaine-binding aptamer MNS-4.1 $\left(\mathrm{K}_{\mathrm{D}} \sim 5 \mu \mathrm{M}\right)$ is structurally stable and forms a TWJ even before binding cocaine ${ }^{78}$ Stojanovic et al. subsequently truncated one of the three stems to destabilize the aptamer, and attached a fluorophore-quencher pair to the ends of the truncated stem for signal reporting. ${ }^{78}$ The resulting aptamer exists in an equilibrium state consisting of both folded and unfolded structures in the absence of the target, in which the fluorophore cannot be efficiently quenched and thus emits strong fluorescence. The aptamer refolds in the presence of cocaine, bringing the quencher into proximity of the fluorophore and resulting in a signal reduction. The method demonstrated a limit of detection (LOD) of $10 \mu \mathrm{M} .^{78}$ The same aptamer sequence has also been modified with electroactive methylene blue for electrochemical detection of cocaine even in the most challenging sample matrix, whole blood. ${ }^{79}$ So far, many structureswitching aptamers have been engineered by truncation for folding-based sensor development. ${ }^{80}$

Traditionally, engineering of structure-switching aptamers with sensitive target response is a laborious multistep process. First, the secondary structure of the fully-folded aptamer must be predicted by software such as mfold or NUPACK. ${ }^{81,82}$ Different domains of the aptamer are then truncated or mutated to identify the target-binding domain and scaffold regions. Finally, multiple truncated aptamers with different portions 
of the scaffold regions removed are synthesized and tested for validating structureswitching functionality, and the optimized aptamer is used for downstream sensor developments. ${ }^{83-85}$ This process is not only labor-intensive but also greatly relies on trial and error. For example, Neves et al. identified the target-binding domain and secondary structure requirements of MNS-4.1 by interrogating 24 truncated/mutated aptamers using isothermal titration calorimetry (ITC), ${ }^{86}$ followed by testing the parent aptamer with three truncated/mutated variants using nuclear magnetic resonance (NMR) to identify the optimized structure-switching aptamer. ${ }^{87}$ To expedite the aptamer engineering process, we have recently developed a one-step nuclease-directed truncation method to generate structure-switching small-molecule-binding aptamers. ${ }^{88}$ Specifically, we found that Exo III digestion of aptamers can be strongly inhibited a few nucleotides prior to their targetbinding domain. The resulting digestion product not only retains substantial targetbinding affinity but also exhibits structure-switching functionality, enabling rapid development of aptamer-based sensors for small-molecule detection.

Since small-molecule-binding aptamers usually have relatively high dissociation constants $(\sim \mu \mathrm{M} \mathrm{K}),{ }^{40}$ a substantial scaffold region is required to stabilize the aptamer structure and maintain target-binding affinity, which promotes problematic background folding. For example, the optimized structure-switching cocaine-binding aptamer described above are partially folded in the absence of target, resulting in high background that greatly limited sensor sensitivity. ${ }^{78,89}$ However, further truncation of this aptamer results in the complete loss of target affinity. ${ }^{86}$ 


\subsubsection{Sensors based on split aptamers}

An alternative way to generate target-induced conformational change is to split the fully folded aptamer into two ${ }^{21}$ or three ${ }^{90}$ fragments, which destabilizes the aptamer such that the fragments are unable to assemble in the absence of target. However, the fragments retain their capacity for target recognition and can successfully reassemble into a complex secondary structure in the presence of target (Figure 2-4C). ${ }^{91}$ As with truncation, splitting of the aptamer also requires knowledge of its target-binding domain and scaffold regions. Splitting is often performed at non-binding loop regions of the aptamer. Recently, Heemstra et al. reported a general approach for engineering smallmolecule-binding DNA split aptamers with TWJ target-binding domains. ${ }^{92}$ Splitting of aptamers with other secondary structures can be challenging, especially for those lacking apparent non-binding loop regions, thus requiring considerable trial-and-error.

Splitting is a more aggressive method that results in reduced background signal compared with truncated aptamers. However, aptamer splitting also results in notably reduced target affinity for the same reason. ${ }^{21}$ Thus, this approach still compromises the sensitivity of the resulting sensors. To improve sensor sensitivity, Zhang et al. reported an amplified assay based on an enzyme-linked split aptamer to perform colorimetric cocaine detection. ${ }^{23}$ One fragment was conjugated to a plastic surface; in the presence of cocaine, this fragment formed a complex with the second fragment, which was modified with biotin in order to bind streptavidin-linked horseradish peroxidase for signal amplification. However, washing caused the dissociation of a subset of the assembled tripartite complexes, resulting in a LOD of just $2.8 \mu \mathrm{M}$. To address this, Heemstra et al. incorporated a proximity ligation 
strategy into this assay. ${ }^{93}$ Here, cocaine binding facilitated the assembly of the azide- and cyclooctyne-modified split fragments, bringing these two chemical groups into close proximity to form covalent bonds. This prevented dissociation of the assembled targetaptamer complex during washing and improved the sensor's detection performance. Although the signal was then amplified by the enzyme, the sensitivity remained limited to $0.1 \mu \mathrm{M}^{94}$ due to the low binding affinity of the split aptamer $\left(K_{D}=\sim 200 \mu \mathrm{M}\right) .{ }^{21}$ Target affinity can be improved by engineering split aptamers with longer complementary stems, but the majority of thermostable split aptamers undergo some degree of pre-assembly in the absence of target, producing high background signal. ${ }^{95}$

To overcome this limitation, we have developed a general approach to incorporate cooperative binding-functionality into split aptamers to achieve more sensitive detection of small molecules..$^{95,96}$ The new aptamer construct termed cooperative binding split aptamer (CBSA) contains two tandem target-binding domains, where the initial targetbinding event stabilizes the structure of the split aptamer and facilitates subsequent target-binding at the second binding domain. Compared with split aptamers with a single target-binding domain, CBSAs exhibit higher target affinity and far more responsive target-induced aptamer assembly, enabling sensitive target detection. For example, we have developed a CBSA-based fluorescence assay that can detect cocaine with a LOD of $50 \mathrm{nM}$ in $10 \%$ saliva within $15 \mathrm{~min}$, which could not be achieved with any conventional split-aptamer-based sensors. ${ }^{95}$

\subsubsection{Sensors based on strand displacement}

Both truncated aptamers and split aptamers require sequence engineering to disrupt the isolated aptamer, but strand-displacement strategies allow for the generation of structure- 
switching aptamers without sequence engineering (Figure 2-4D).${ }^{97}$ This strategy employs a short competitive strand complementary to the aptamer. In the absence of the target, the competitive strand hybridizes with the aptamer and disrupts its folded structure. The aptamer undergoes a conformational change upon target binding, refolding into its original structure and thus dissociating with the competitive strand. This target-induced structure-switching event can be readily transduced into an optical ${ }^{97}$ or electrochemical ${ }^{22}$ signal. For example, Li et al. attached a fluorophore to an ATP-binding aptamer and a quencher onto the competitive strand. In the absence of the target, the hybridization of aptamer and the competitive strand brings the fluorophore into proximity with the quencher, yielding low fluorescence. Upon addition of the target, the competitive strand is displaced, resulting in a high fluorescent signal. ${ }^{97}$ Since no sequence engineering is required in the strand-displacement approach, less knowledge is required about the aptamer structure. Moreover, library-immobilized SELEX methods have been developed that make it possible to isolate aptamers with inherent structure-switching functionality, ${ }^{32,98}$ which further simplify sensor development. Nevertheless, since competitive strands have high binding affinity for the aptamer, they inadvertently inhibit target binding and consequently reduce the sensitivity of strand-displacement assays. This is particularly problematic for the detection of small-molecule targets, which have relatively low target-binding affinity. As such, the LOD of strand-displacement assays for small molecules is usually comparable to or even higher than the $\mathrm{K}_{\mathrm{D}}$ of the aptamer being employed. ${ }^{32,33,97,99,100}$ Moreover, unlike truncated aptamer or split-aptamer-based assays, strand-displacement assays require a heating-and-cooling process lasting $\geq 30 \mathrm{~min}$ to 
ensure complete hybridization between the complementary strand and aptamer to achieve low background. This greatly hinders the use of these assays for rapid on-site detection.

\subsubsection{Sensors that do not require target-induced conformational change}

Recently, several methods have been developed that can generate a readout of target binding without utilizing a structure-switching aptamer. These typically employ a fullyfolded aptamer in combination with a second probe such as a dye molecule, exonuclease, or even the target itself to report target-binding events. This eliminates the need for laborious sequence engineering but also yields high sensitivity due to the high targetbinding affinity of fully-folded aptamers. The need for specific signaling probes is the major drawback of these methods, which has limited their application to only a few targets. Nevertheless, several new strategies have been developed to improve the generality of these methods.

\subsubsection{Dye-displacement assays}

Dye-displacement assays ${ }^{34,74,101-104}$ rely on small-molecule dyes that can initially be associated with the aptamer-binding domain (Figure 2-4E). The target displaces the dye from the binding domain, resulting in a change in the absorbance or fluorescence of the dye. These assays do not require any additional labeling or chemical modification of the aptamer. Since the aptamers typically bind to these small-molecule targets and dyes with similar affinities, target-induced dye-displacement is more thermodynamically feasible than the displacement of a tightly-bound complementary strand in strand-displacement assays. As a result, dye-displacement assays can achieve a LOD which is 10-fold lower than the $\mathrm{K}_{\mathrm{D}}$ of the aptamer being used. ${ }^{34,74,101-104}$ However, this assay strategy has not been generalized, as there are currently no sequence engineering strategies or SELEX 
techniques that can directly isolate aptamers with dye-displacement functionality. To remedy this problem, we recently developed a simple strategy for directly isolating signal-reporting aptamers that have intrinsic dye-displacement functionality from a TWJ structured DNA library. ${ }^{34}$ As a demonstration, we isolated an aptamer against a synthetic cathinone, 3,4-methylenedioxypyrovalerone (MDPV). The aptamer intrinsically binds the small-molecule dye Cy7 within its TWJ domain, while MDPV efficiently displaces the dye, resulting in a change in absorbance within seconds. The assay also showed high sensitivity, achieving a LOD of $300 \mathrm{nM}$ in $50 \%$ urine or saliva. ${ }^{34}$

\subsubsection{Aptamer-modified field-effect transistors}

Receptor-modified field-effect transistors (FETs) have recently gained attention in the biosensing field. ${ }^{105}$ The conductivity of the semiconductor channel in FETs is extremely sensitive to changes in the local electric field. When the surface of a FET is modified with a specific bioreceptor such as an aptamer, binding of charged target molecules at the surface is sufficient to generate an electric signal. ${ }^{105}$ FET-based sensors can achieve ultrahigh sensitivity, partially because of the nonlinear behavior of the semiconductor channel. Since the charge on the targets is being employed for signal transduction, highaffinity fully-folded aptamers can be directly employed in the sensor platform. Moreover, FET offers a broad dynamic range spanning several orders of magnitude, which cannot be achieved in most sensing platforms. ${ }^{106}$ For example, Weiss et al. demonstrated detection of positively-charged dopamine using an aptamer-modified FET with a LOD of $10 \mathrm{pM}$ and a dynamic range from $10 \mathrm{pM}$ to $1 \mu \mathrm{M} \cdot{ }^{107}$ Aptamer-modified FETs do have some significant limitations, however. First, small-molecule targets with no charge have minimal effect on the semiconductor, and thus cannot be effectively measured. ${ }^{108}$ Second, 
semiconductor transconductance is only sensitive to variations in charge density within the electrical double layer, but the thickness of this layer dramatically decreases as solution ionic strength increases-dropping below $1 \mathrm{~nm}$ in physiological fluids, which makes target detection challenging in these conditions. ${ }^{109}$ Recent work has shown the possibility of remedying both limitations, based on the determination that small-molecule binding can introduce a ternary structure change in fully-folded aptamers modified onto the FET surface. This rearrangement of negatively-charged aptamer phosphodiester backbones near the semiconductor surface can then be detected by FET even if the binding event itself occurs outside the electrical double layer. Using this strategy, Andrews et al. demonstrated sensitive detection of both charged and non-charged small molecules in biological samples. ${ }^{110}$ The generalizability of this strategy for other smallmolecule-binding aptamers needs to be assessed in the future, however. This will not be easy due to the need for specialized equipment and techniques for fabrication of aptamermodified FETs, which are not accessible for most labs.

\subsubsection{Exonuclease-inhibition assay}

We have recently discovered that binding of small-molecules to aptamers can strongly affect their digestion by the exonucleases Exonuclease III (Exo III) and Exonuclease I (Exo I) ${ }^{88,111}$ Exo III and Exo I respectively digest double- and single-stranded DNA from the $3^{\prime}$ terminus. In the absence of target, aptamers can be completely digested by this nuclease mixture. However, upon binding of the target, digestion by both enzymes is strongly inhibited at a position 3-4 nucleotides prior to the target-binding domain, yielding a truncated aptamer. Such drastic differences in digestion profiles can be utilized to determine the presence of the target using a DNA-binding dye such as SYBR gold or a 
fluorophore-quencher-modified molecular beacon. This exonuclease-based assay allows for the use of high-affinity fully-folded aptamers without any sequence engineering or modification, achieving much higher sensitivity compared with sensors based on structure-switching aptamers. We have demonstrated the generality of this method using different aptamers with both TWJ and stem-loop secondary structures, with low nanomolar detection limits in biological matrices. ${ }^{111}$ Since each aptamer has a unique sequence, multiplex detection can also be achieved in a single-pot reaction using specific molecular beacons (Figure 2-5).

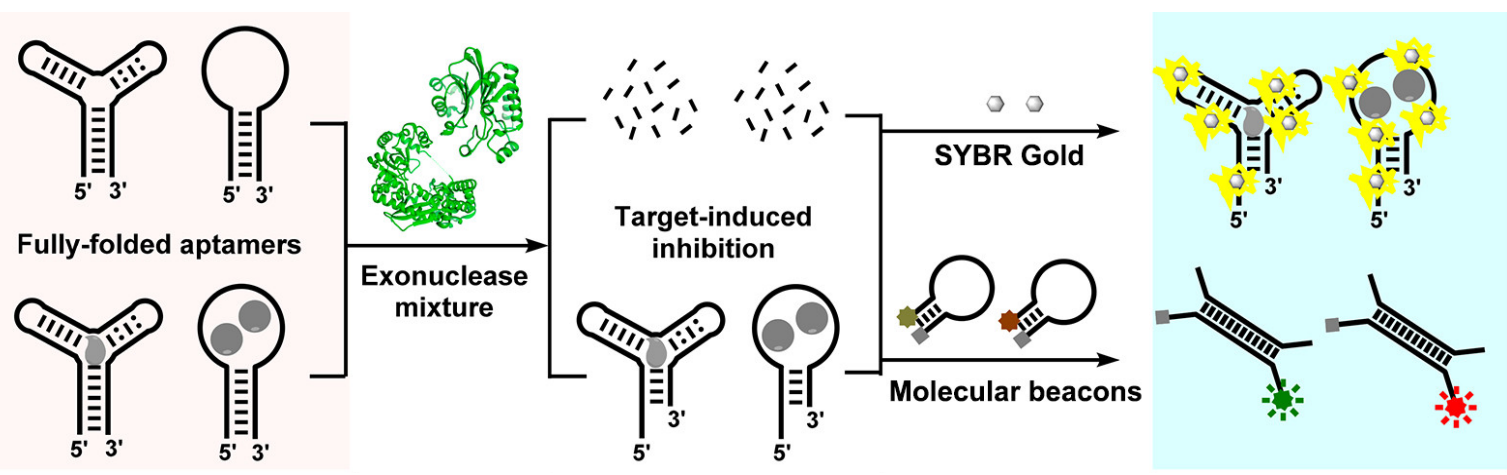

Figure 2-5. Working principle of exonuclease-inhibition assay for small molecule detection. ${ }^{111}$

\subsection{Gold nanoparticles (AuNPs) and their use in aptamer-based sensors}

AuNPs are gold particles with diameters ranging from 1-100 nm, and are widely used for biosensor development due to their distinctive optical properties. The color of an AuNP solution is determined by their localized surface plasmon resonance (LSPR) and can thus change according to their size, shape, and distance from each other. For example, 13-nm spherical citrate-capped AuNPs have strong absorbance at $520 \mathrm{~nm}$ and present a red color. Particles with smaller sizes have higher LSPR frequency, resulting in a blue-shift of their absorbance spectra that produces an orange-red color, whereas larger particles absorb at longer wavelengths and thus produce a purple color. When AuNPs 
aggregate upon addition of salt, the absorbance peak shifts to $650 \mathrm{~nm}$ and the solution color changes to blue. The color changes that occur upon dissolution, enlargement, assembly, dissociation, or aggregation of AuNPs have been extensively utilized for signal transduction in different sensors for the instrument-free, naked-eye detection of various targets. In addition, nucleic acids can be easily modified onto AuNPs through gold-thiol chemistry. For these reasons, AuNPs have been widely used as a sensing element for colorimetric detection of targets including metal ions, small molecules, DNA, and proteins. ${ }^{112}$

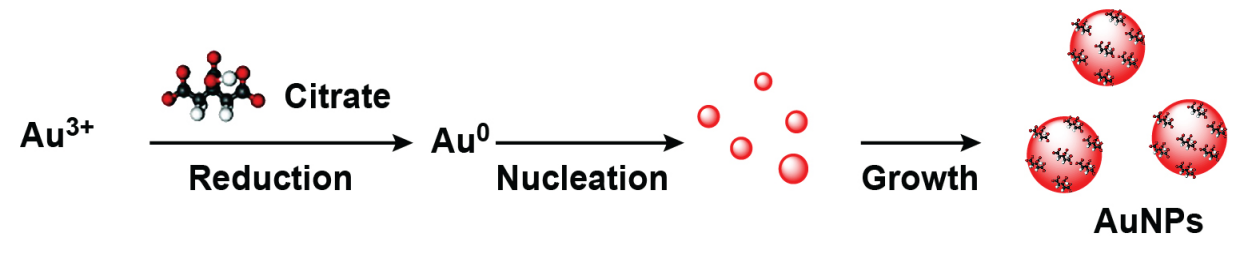

Figure 2-6. Synthesis AuNPs by citrate reduction.

\subsubsection{Synthesis and characterization of citrate-capped AuNPs}

Various techniques have been developed for the synthesis of AuNPs with different sizes and shapes. ${ }^{113-117}$ The most commonly used technique is the Turkevitch method, which uses citrate to reduce $\mathrm{HAuCl}_{4}$ into spherical AuNPs in aqueous solution (Figure 2-6). ${ }^{113} \mathrm{HAuCl}_{4}$ solution is mixed with trisodium citrate; $\mathrm{Au}^{3+}$ ions in the solution are first reduced to $\mathrm{Au}^{+}$ions, and then to $\mathrm{Au}^{0}$ species through disproportionation. This $\mathrm{Au}^{0}$ species is thermodynamically unstable and rapidly becomes a nucleation center for other newly-reduced $\mathrm{Au}^{0}$ species. These nucleation centers therefore slowly grow into welldispersed AuNPs. Excessive citrate anion acts as a capping reagent that adsorbs onto the surface of the AuNPs and renders them negatively-charged. These AuNPs therefore repel each other and are stable for a long period of time without aggregation. The size of the 
AuNPs can be easily fine-tuned by adjusting the $\mathrm{HAuCl}_{4}$ :citrate ratio, temperature, or solution $\mathrm{pH} .{ }^{113}$

After synthesis of AuNPs, the size, shape, concentration, and dispersity of the nanoparticles can be characterized by various methods (Figure 2-7). Given the color change associated with these particles (Figure 2-7A), one can easily determine their size and concentration based on UV-vis absorbance (Figure 2-7B). The surface plasmon peak wavelengths and extinction coefficients for different sizes of AuNP have been well studied, ${ }^{118}$ and UV-vis spectrometry offers a rapid, facile, and non-destructive way to characterize the size and concentration of synthesized AuNPs. The accuracy of this method is relatively low, however - especially for size determination. This is because the surface plasmon peak wavelength of AuNPs with sizes ranging from 1 to $100 \mathrm{~nm}$ only varies between 520 and $580 \mathrm{~nm},{ }^{118}$ and the local environment and different surface modifications have been known to further affect their UV-vis absorbance. ${ }^{119} \mathrm{UV}$-vis spectrometry is also inaccurate for poly-disperse AuNPs due to the overlapping surface plasmon peaks from particles of different sizes. Dynamic light scattering (DLS) is another analytical method routinely used to characterize the size and dispersity of AuNP solutions (Figure 2-7C). ${ }^{119}$ Nanoparticles in the solution scatter light upon illumination, and the intensity of scattered light rapidly fluctuates over time due to Brownian motion of the particles. Smaller particles have faster Brownian motion, which results in more rapid DLS fluctuation, thus allowing accurate measurement of particle size. DLS measurement also provides information about particle dispersity, which is important for assessing the quality of AuNPs. ${ }^{119}$ Notably, DLS measures the hydrodynamic size of particles, which includes the protectant (such as citrate) and surface modification (such as DNA) instead 
of just the size of the gold core. This unique feature of DLS allows the study of bioconjugation processes on AuNPs. ${ }^{120}$ Finally, transmission electron microscopy (TEM) is a powerful tool for AuNP characterization (Figure 2-7D). Although it requires much more expensive instruments and more complicated sample preparation, the size of individual AuNPs can be accurately determined without the interference of solvent conditions or surface protectants/modifications. Moreover, TEM also gives direct information on particle shape that cannot be obtained using other methods.
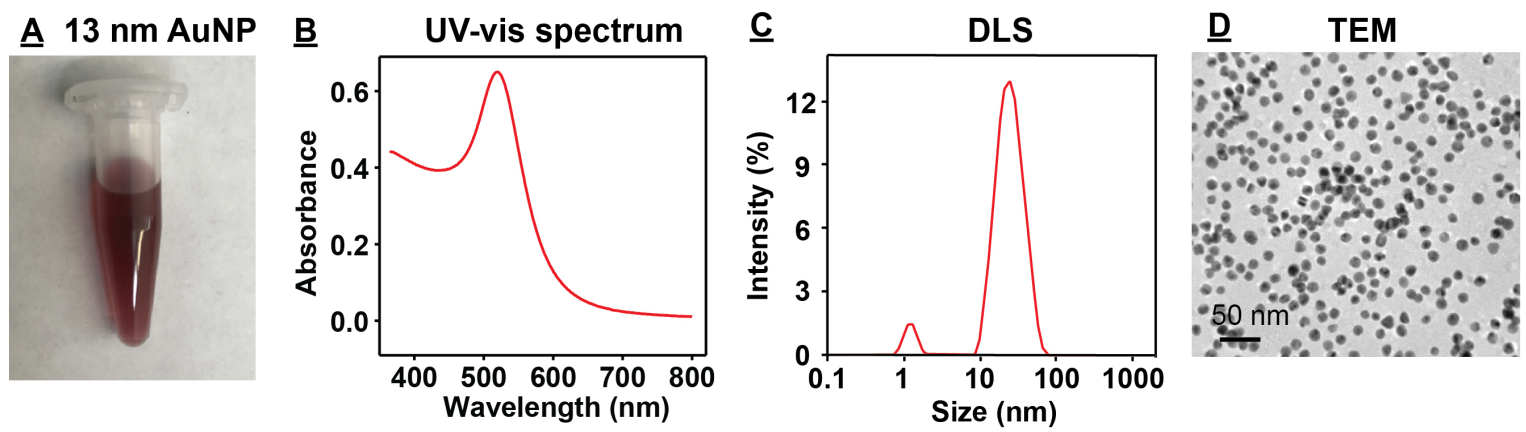

Figure 2-7. Methods for AuNP characterization. (A) color, (B) UV-vis spectrum, (C) DLS measurement, and (D) TEM picture of $13 \mathrm{~nm}$ AuNPs.

\subsubsection{DNA functionalized AuNPs}

DNA-modified AuNPs can specifically recognize a variety of targets, including nucleic acids, proteins, peptides, small molecules, and metal ions. ${ }^{12}$ Accordingly, such particles have proven extraordinarily useful for bioimaging, bioseparation, diagnostic assays, drug targeting, nanotherapeutics, and nanomaterial assembly. ${ }^{10,121,122}$ DNA can be conjugated onto AuNPs by several chemistries, ${ }^{123-126}$ where alkane-thiol adsorption is the most common approach (Figure 2-8). ${ }^{125,127}$ In this method, an excessive amount of thiolated DNA is mixed with AuNPs for several hours to allow gold-thiol interaction. The DNA surface coverage on AuNPs is controlled by the DNA:AuNP ratio employed for modification. Next, salt aging is performed, in which the salt concentration of the AuNP- 
DNA solution is gradually increased. Citrate-capped AuNPs without DNA attached are not stable at elevated ionic strength and aggregate from the solution, while conjugation of highly negatively charged-DNA allows modified AuNPs to tolerate even $1 \mathrm{M}$ salt concentrations. ${ }^{128}$ Finally, the DNA-modified AuNPs are washed with buffer by ultrafiltration or ultracentrifugation to remove salt and unconjugated DNA. The resulted DNA-modified AuNPs can then be characterized using different methods and can be stored refrigerated for several months.

The extent of DNA surface coverage can profoundly influence DNA hybridization efficiency,_enzyme activity, and assay sensitivity employing DNA-modified AuNPs. ${ }^{129-}$ ${ }^{135}$ High surface coverage is associated with steric hindrance, electrostatic repulsive interactions, and elevated surface salt concentration, whereas low surface coverage can result in nonspecific binding of oligonucleotides to the particle surface. Both scenarios can greatly reduce DNA hybridization efficiency and enzyme activity for surface-bound DNA probes, ${ }^{136,137}$ resulting in low sensitivity of DNA-modified AuNP-based assays. Therefore, strategies for accurately quantifying the degree of surface coverage are critical for research applications based on DNA-conjugated particles.

DNA surface coverage on AuNPs under different modification conditions is typically quantified using thiolated DNA with a fluorophore label. Fluorophore-labeled DNA is strongly quenched when bound to AuNPs, but this fluorescence is fully recovered once the DNA is detached from the surface. Therefore, the surface coverage of thiolated-DNAmodified AuNPs can be determined by measuring the fluorescence of oligonucleotides liberated via ligand displacement with dithiothreitol (DTT), which can effectively bind AuNPs via two thiol groups. ${ }^{138,139}$ The standard DTT displacement protocol usually 
requires overnight incubation of DNA-modified AuNPs with high concentrations ( $\sim 0.5$ M) of DTT at room temperature. ${ }^{128}$ Although time-consuming, the DTT displacementbased assay is recognized as a reliable "gold standard" for quantifying surface coverage on AuNPs irrespective of DNA length or sequence. Recently, several label-free methods utilizing PCR, OliGreen, and unimolecular beacon have been developed to characterize DNA surface coverage. ${ }^{140-142}$

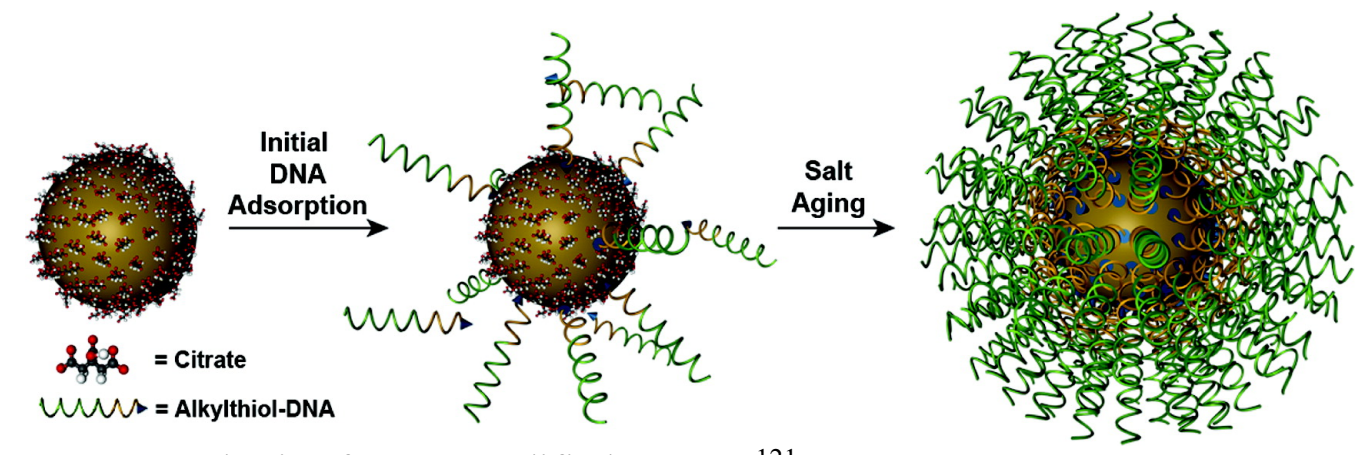

Figure 2-8. Synthesis of DNA-modified AuNPs. ${ }^{121}$

We have recently developed a simple and broadly applicable Exo III digestion assay based on the cleavage of phosphodiester bonds - a universal feature of DNA-modified particles - to accurately quantify DNA probe surface coverage on AuNPs and other commonly used particles of different compositions, conjugation chemistries, and sizes. ${ }^{143}$ Our assay utilizes particle-conjugated, fluorophore-labeled probes that incorporate two abasic sites. These probes are hybridized to a cDNA strand, and quantitation is achieved via cleavage and digestion of surface-bound probe DNA via Exo III's apurinic endonucleolytic and exonucleolytic activities. The presence of the two abasic sites in the probe greatly speeds up the enzymatic reaction without altering the packing density of the probes on the particles. Probe digestion releases a signal-generating fluorophore and liberates the intact cDNA strand to start a new cycle of hybridization and digestion until 
all fluorophore tags have been released. After digestion, DNA surface coverage can be determined accurately based on the complete release of fluorophores. Our method delivers accurate, rapid, and reproducible quantitation of thiolated DNA on the surface of gold nanoparticles, and also performs equally well with other conjugation chemistries, substrates, and particle sizes, and thus offers a broadly useful assay for quantitation of DNA surface coverage.

\subsubsection{Aptamer-based assays employing AuNPs for small molecule detection}

Both unmodified and aptamer-modified AuNPs have been widely employed as colorimetric signal reporters in aptamer-based assays for naked-eye small-molecule detection. ${ }^{96,144-146}$ Unmodified citrate-capped AuNPs strongly adsorb single-stranded DNA through interaction with exposed nitrogenous bases, but have low binding affinity to double-stranded DNA. ${ }^{147}$ Meanwhile the binding of negatively-charged singlestranded DNA to AuNPs significantly stabilizes AuNPs against salt-induced aggregation. ${ }^{148}$ Basing on this feature, Fan et al. developed a simple split-aptamer-based assay for rapid visual detection of cocaine (Figure 2-9A). ${ }^{145}$ A cocaine-binding split DNA aptamer was incubated with 13-nm citrate-coated AuNPs, followed by addition of the analyte and a salt solution. In the absence of target, both split aptamer fragments are in a single-stranded conformation and adsorb onto the AuNPs. The DNA-protected AuNPs are stable with the addition of salt solution, and thus the sample maintains the red color of the original AuNP mixture. In the presence of target, however, the split-aptamer fragments assemble, forming a duplexed complex that detaches from the particle surface. When a salt solution is added into the sample, the unprotected AuNPs rapidly aggregate and produce a clearly visible red-to-blue color change. Using this method, cocaine was 
visually detected within 10 min at concentrations as low as $20 \mu \mathrm{M}$. Similar methods have been developed for the detection of other targets such as $\mathrm{ATP}^{145}$ and aminoglycosides ${ }^{36}$. Recently, the same method has also been successfully applied to a truncated structureswitching aptamer for detection of cocaine. ${ }^{149}$ Unfortunately, these assays are not very specific, as unmodified AuNPs are not stable and can undergo non-specific aggregation under high salt or target concentration, even in the presence of single-stranded DNA. ${ }^{128}$

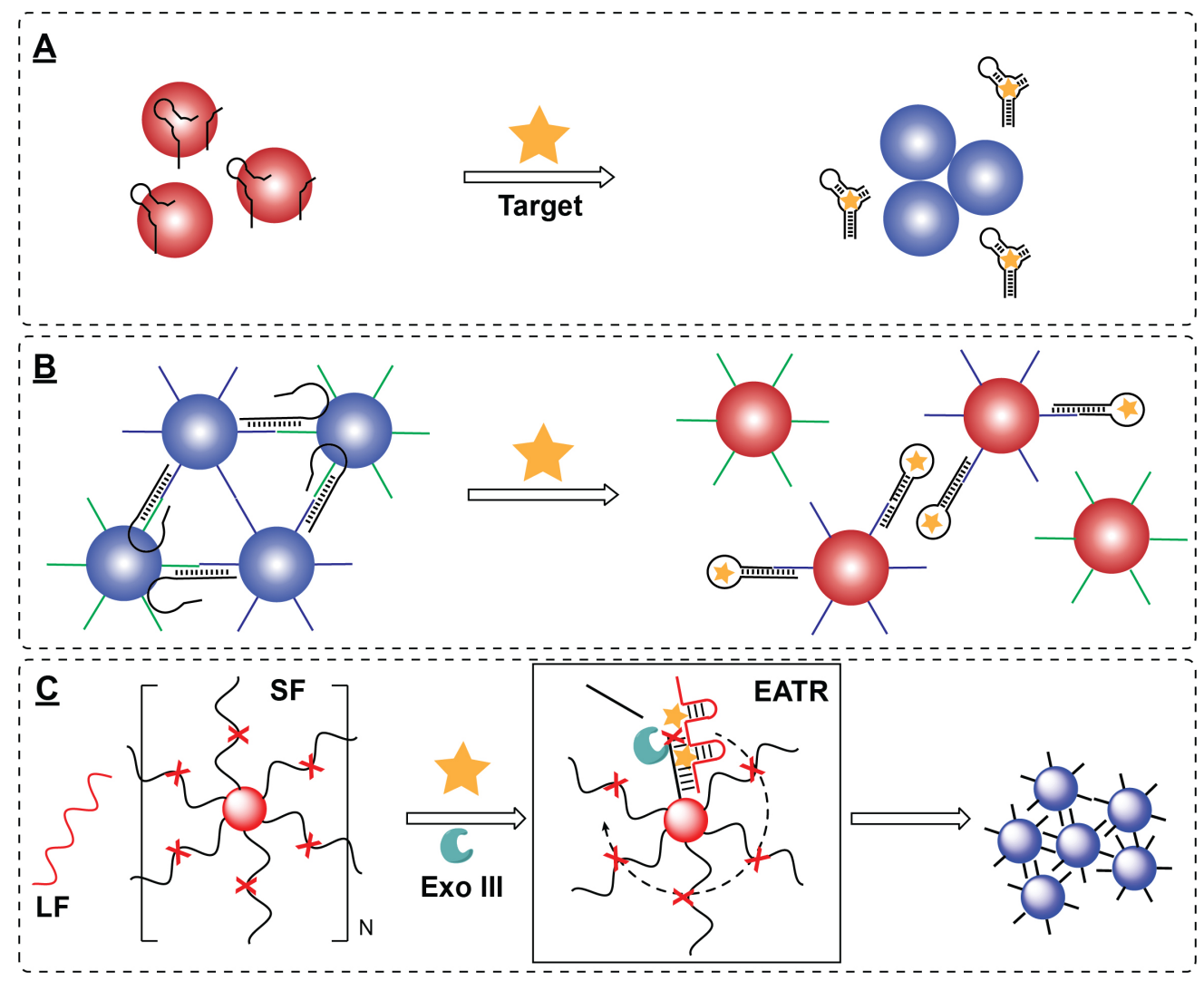

Figure 2-9. Examples of aptamer-based colorimetric assays employing AuNPs for small molecule detection. (A) Assay based on unmodified AuNPs. (B) Assay based on DNA- modified AuNPs employing a "assembly-to-dissociation" strategy. (C) Assay based on CBSA- modified AuNPs employing a "dissociation-to-aggregation" strategy.

On the other hand, DNA-modified AuNPs are stable in high-salt buffers because of repulsion by the negatively-charged DNA strands tethered to the particle surface. ${ }^{128}$ They can also undergo programmable dissociation or aggregation upon specific target-binding, 
yielding a colorimetric readout for sensitive target detection. For example, Li et al. discovered that adenosine-binding aptamers conjugated onto 13-nm AuNPs with low surface density collapse onto the surface in the absence of the target, but fold into a rigid duplexed structure that lifts up from the surface upon target binding. Such a targetinduced conformational change alters the inter-particle steric hindrance and prevents AuNPs from aggregating under high ionic strength. ${ }^{150}$ Using this method, adenosine was detected at concentrations as low as $20 \mu \mathrm{M}$. It is also well known that two sets of AuNPs modified with different DNA probes can assemble into lattice-like nano- and microstructures in the presence of a cross-linking strand that is complementary to both surfacemodified probes, producing a blue color. ${ }^{125,151}$ This process is reversible at elevated temperatures when the linker strand dissociates from the surface-modified DNA, producing a blue-to-red color change. If the cross-linking strand is replaced with a structure-switching aptamer, AuNP assembly and dissociation can be mediated by target concentration (Figure 2-9B). Specifically, in the absence of target, the aptamer unfolds and links two sets of DNA-modified AuNPs together, forming blue aggregates. In the presence of target, the aptamer folds into a duplexed structure and dissociates from the surface-immobilized probes, resulting in dissociation of the aggregates and producing a blue-to-red color change. Using this "assembly-to-dissociation" method, Lu et al. demonstrated aptamer-based visual detection of cocaine and adenosine. ${ }^{144}$

Another commonly-employed strategy known as "dissociation-to-aggregation" utilizes an target-triggered enzymatic cleavage reaction to remove surface-bound DNA strands, which destabilizes the AuNPs and causes them to aggregate, producing a red-to-blue color change (Figure 2-9C) ${ }^{96,152-155}$ For example, we recently demonstrated the use of 
CBSAs for sensitive colorimetric detection of cocaine based on the aggregation of AuNPs via enzyme-assisted target recycling (EATR). ${ }^{96,155}$ We coupled one the two CBSA fragments to AuNPs while the other remained free in solution. In the absence of target, the fragments remain separate, and the AuNP-conjugated fragment is resistant to digestion by Exo III. When the target is present, the CBSA fragments assemble, forming a substrate that can be cleaved by Exo III. This digestion shears all surface-bound DNA, resulting in aggregation of AuNPs and a red-to-blue color change. This method allowed for visual detection of cocaine at concentrations as low as $10 \mu \mathrm{M}$ within 30 minutes. ${ }^{96}$

\subsection{EATR-based signal amplification}

In conventional bioassays, assay sensitivity is limited by the the target affinity of the bioreceptor, as the signal intensity is proportional to the number of receptor-bound target molecules. ${ }^{156}$ To overcome this limit, many amplification approaches have been developed that enable detection of targets at very low concentrations ${ }^{157,158}$. EATR ${ }^{159}$ relies on selective, nuclease-mediated degradation of the probe strand of a target-probe duplex that only forms in the presence of target. This liberates the target, which is then 'recycled' for use in further digestion reactions. In this scenario, each target molecule can generate multiple signals, achieving much higher sensitivity compared with assays without amplification. So far, many EATR-based assays have been developed for the detection of DNA as well as small molecules. ${ }^{96,159,160}$

\subsubsection{EATR assays for DNA detection}

EATR was first used with molecular beacon probes for DNA detection (Figure 2-10). ${ }^{158}$ The molecular beacon probe contains a loop sequence complementary to the target DNA, with a fluorophore-quencher pair attached to the stem of the beacon as a 
signal-reporting element. When the molecular beacon probe binds the target DNA, it switches from a stem-loop structure into a probe-target duplex that contains a specific sequence that can be recognized by a nicking enzyme. This enzyme then catalyzes cleavage of the beacon probe, liberating the fluorophore and releasing the target, which can then be recycled to hybridize with other molecular beacon probes. Thus, an amplifiedfluorescent signal can be generated by a single copy of the target though EATR, greatly decreasing the LOD. Using this EATR method, termed nicking enzyme signal amplification (NESA), Xie et al. demonstrated more than 100-fold signal amplification and achieved a LOD of $6.2 \mathrm{pM}$ for a DNA target. ${ }^{158}$ Without the assistance of the enzyme, the assay remained insensitive to target concentrations as high as $2 \mathrm{nM}$. Given that DNA can be easily modified with various signaling reporters ${ }^{154,158,161,162}$ and different nucleases can be utilized to process target-probe duplexes, ${ }^{158,161,163,164}$ many EATR-based assays have been developed for DNA detection with ultra-high sensitivity. ${ }^{159}$ For example, Plaxco et al. improved the NESA assay by replacing the nicking enzyme with a structure-specific nuclease, Exo III. This method does not require a specific enzyme-recognition sequence in the target, and thus can be generalized for detection of virtually any sequence. ${ }^{164}$ More recently, we have successfully demonstrated EATR on the surface of AuNPs and achieved ultra-sensitive visual detection of DNA targets. ${ }^{154}$ The assay utilizes particle-conjugated DNA probes that incorporate two abasic sites. The single-stranded probe is inert to Exo III, rendering the AuNPs stable in solution and producing a red color. Target DNA can hybridize to the AuNP-conjugated probes forming a stable duplex, and these duplexes can then be rapidly digested by Exo III. Probe digestion releases the target strand to start new cycles of hybridization and 
digestion until all surface-tethered probes are sheared. The AuNPs then aggregate, yielding a red-to-blue color change. This color change is easily observed by the naked eye within $15 \mathrm{~min}$, and as little as $2 \mathrm{nM}$ of target can be detected using this method. Importantly, even a single-base target mismatch adjacent to the abasic sites impairs Exo III activity on the probe, making this assay suitable for single nucleotide polymorphism detection.

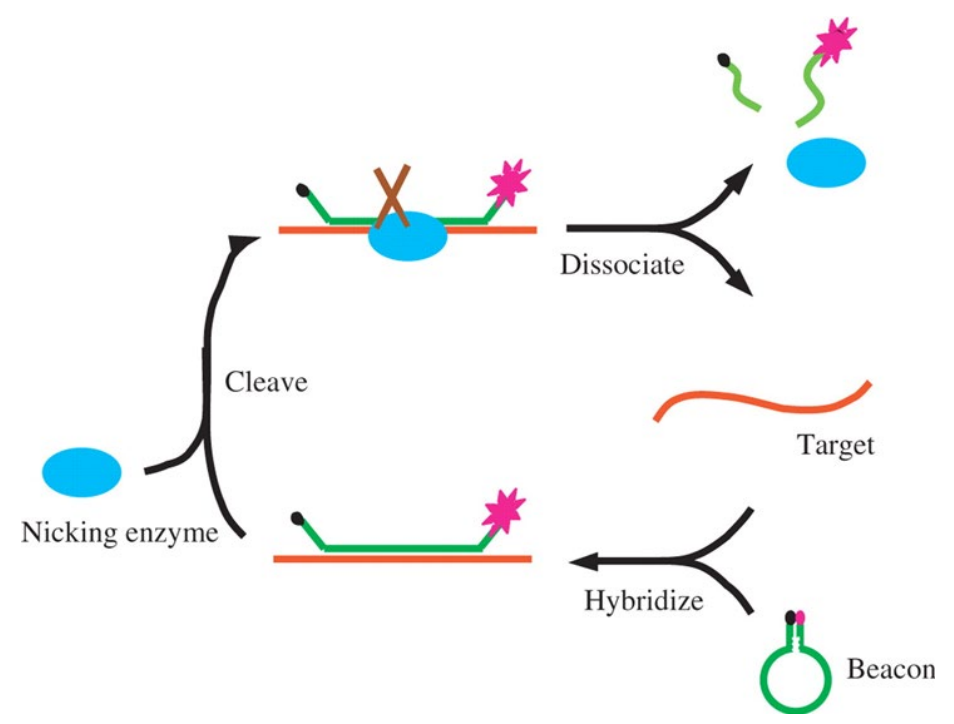

Figure 2-10. Working principle of an EATR assay for detection of DNA. ${ }^{158}$

\subsubsection{Aptamer-based EATR assays for small-molecular detection}

In principle, the target-induced assembly of split aptamers should be compatible with EATR-mediated signal amplification, since this process largely resembles hybridization between target and probe strands in DNA-based EATR assays. However, such an approach has been historically challenging due to the low target affinity and insensitive target-induced aptamer assembly of small-molecule-binding aptamers. We have recently overcome these limitations by incorporating highly target-responsive CBSAs into EATRamplified biosensors. Specifically, we have developed an EATR-amplified fluorescence assay for the detection of dehydroepiandrosterone sulfate (DIS) using a fluorophore- 
quencher-labeled CBSA and Exo III, and have demonstrated that this assay exhibits 100fold enhanced sensitivity relative to the CBSA alone without EATR signal amplification. Importantly, this assay also proved highly sensitive when used in urine samples, allowing us to detect DIS at concentrations as low as $1 \mu \mathrm{M}$ in $50 \%$ urine within 30 minutes. Moreover, using CBSA fragment-modified AuNPs as signal reporters, naked-eye detection of low micromolar concentrations of cocaine was achieved within 20 minutes.

\subsection{Small molecule targets used in this work}

\subsubsection{Cocaine}

Cocaine (Figure 2-11A) is a strong and highly addictive central nervous system stimulant extracted from the coca plant. Cocaine is currently a schedule II-controlled substance regulated by the Drug Enforcement Agency, and has been approved for limited medical uses. Recreational abuse of cocaine can have severe negative health consequences, such as nausea, hyperthermia, hypertension, cardiac arrest, and even

death. ${ }^{165}$ Cocaine is the second most abused and third most frequently seized illicit drug in the United States. ${ }^{166}$ The National Survey on Drug Use and Health estimated that in 2016, about 1.9 million people aged 12 or older were current users of cocaine. ${ }^{167}$

\subsubsection{DIS}

DIS (Figure 2-11B), also known as dehydroepiandrosterone sulfate (DHEA-S) sulfate is a conjugated metabolite of DHEA, which is the precursor of many other androgens and estrogens. ${ }^{168}$ DIS is one of the most abundant steroid hormones in both blood and urine. DIS itself has no hormonal activity; however, it can be converted into hormonally-active DHEA by steroid sulfatase. Upon secretion by adrenal cortex into the circulation, DIS is mostly bound to serum albumin, and thus has a long biological half-life of 14 hours. ${ }^{168}$ 
DIS is eventually excreted into the urine through the kidney. DIS concentration in blood and urine reflects adrenal gland function. Levels are low during childhood, rapidly increase during sexual maturation, and peak around the age of 20 to 30 years and gradually decrease afterward. ${ }^{169}$ Urinary concentration of DIS in healthy adults typically ranges from $1-20 \mu \mathrm{M}^{170,171}$ but can climb to the sub-millimolar range in patients with adrenal dysfunction and adrenal tumors. ${ }^{172}$

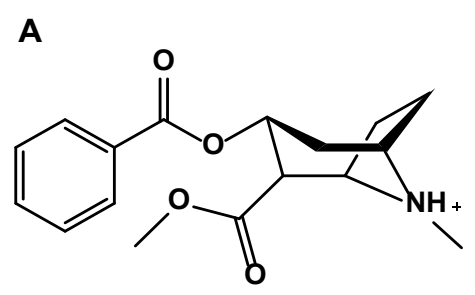

Cocaine

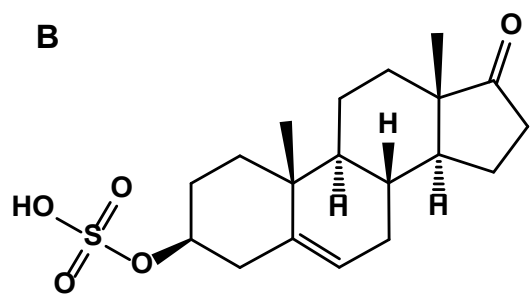

DIS<smiles>[R]c1ccc(C(=O)C([R2])N([R])[R])cc1</smiles>

Synthetic Cathinones

Figure 2-11. Chemical structure of small molecule targets. Structures of (A) cocaine, (B) DIS, and $(\mathbf{C})$ core structure of synthetic cathinones are shown. R1-R4 represent substituent sites.

\subsubsection{Synthetic cathinones}

Synthetic cathinones (Figure 2-11C), known popularly as 'bath salts', are a family of novel psychoactive substances that share the same beta-keto phenethylamine chemical core structure. They are highly addictive central nervous system stimulants, ${ }^{173}$ and are associated with many negative health consequences, such as agitation, seizures, cardiac arrest, and even death. ${ }^{174,175}$ Synthetic cathinones are sold on the Internet and in head shops as "legal" alternatives to amphetamines and ecstasy. ${ }^{19}$ Evidence from poison control centers suggests that synthetic cathinones are primarily used by youths and young adults. ${ }^{176}$ So far, 14 synthetic cathinones have been scheduled as controlled substances by the Drug Enforcement Agency. ${ }^{177}$ However, in attempts to circumvent existing drug laws, drug designers continually add new substituents to existing synthetic cathinones, giving 
rise to several hundred novel synthetic cathinone derivatives that are potentially even more potent and dangerous. ${ }^{19}$ Synthetic cathinones thus pose a major risk to public health and safety due to their severe and unpredictable toxicity, the difficulty of achieving effective surveillance, and their increasingly broad availability. 
CHAPTER 3: A Cooperative-Binding Split Aptamer Assay for Rapid, Specific and Ultra-Sensitive Fluorescence Detection of Cocaine in Saliva

\subsection{Introduction}

Aptamers are single-stranded oligonucleotides isolated via in vitro SELEX, ${ }^{1,2}$ which can specifically bind to a wide range of targets including proteins, small molecules and metal ions. ${ }^{3}$ Aptamers offer several advantages as recognition elements for biosensors relative to antibodies. First, aptamers can be chemically synthesized with high reproducibility at relatively low $\operatorname{cost}^{5}$ Second, the high chemical stability of DNA aptamers means that they can be used under harsher conditions and stored with a longer shelf life. ${ }^{5}$ Finally, it is possible to generate unstructured aptamers that form specific secondary structures such as three-way junctions $s^{33,78}$ or tertiary folds such as Gquadruplex structures ${ }^{8,29}$ upon target binding. Such target-induced conformational changes can be readily exploited for specific target detection in a variety of applications, including medical diagnostics, environmental monitoring and drug screening. ${ }^{9-11}$

However, target-induced conformational change is hard to control, especially for small-molecule-binding aptamers that have relatively high dissociation constants $(\sim \mu \mathrm{M}$ $\left.\mathrm{K}_{\mathrm{D}}\right) .{ }^{40}$ For example, the well-characterized cocaine-binding aptamer MNS-4.1 $\left(\mathrm{K}_{\mathrm{D}} \sim 5\right.$ $\mu \mathrm{M})$ is structurally stable and forms a three-way junction even before binding cocaine. ${ }^{101}$ To achieve an effective target-induced conformational change, the aptamer can be split into two $^{21}$ or three ${ }^{90}$ fragments. This destabilizes the aptamer such that the fragments are unable to assemble without target, while the fragments retain their capacity for target recognition and can successfully reassemble into a complex secondary structure in the presence of target. Such target-induced assembly of the split aptamers can accordingly be 
employed for signal transduction. However, aptamer splitting results in notably reduced target affinity. ${ }^{21}$ Thus, this approach still compromises the sensitivity of split aptamerbased sensors, even with various amplification strategies applied..$^{23,93}$

To overcome the limitation of split aptamers, we have developed a cooperative binding-based approach to generate split aptamers that retain high target affinity. Cooperative binding behavior is commonly observed in ligand-binding proteins that are highly responsive to ligand concentration, such as hemoglobin, ${ }^{178}$ ion channels ${ }^{179}$ and transcription factors. ${ }^{180}$ Those proteins generally have more than one ligand-binding site, where binding at one site increases the affinity of the other sites, resulting in a 'switchlike' binding curve. ${ }^{181}$ Breaker et al. initially found that some tandem riboswitches ${ }^{182,183}$ naturally employ such cooperative binding ${ }^{182}$ to control gene expression in response to subtle changes in ligand concentration. This cooperative behavior was further extended into artificial biosystems such as ribozymes, ${ }^{184}$ molecular beacons ${ }^{185}$ and DNA aptamers ${ }^{186}$. Specifically, Plaxco et al. introduced the disorder into the parent aptamer to achieve cooperative binding, and focused on the demonstration of the "switch-like" response behavior of cooperative binding that occurred when ligand concentration approaches $\mathrm{K}_{1 / 2}\left(\mathrm{~K}_{1 / 2}\right.$ represents the ligand concentration at which half of the binding domains are occupied). These engineered cooperative DNA aptamers could not employ for practical sensor platform because the introduction of cooperativity unavoidably reduced the target-binding affinity of the resulting cooperative aptamer $\left(K_{1 / 2}=\sim 3\right.$ $\mathrm{mM}) .{ }^{186}$

We have for the first time successfully incorporated two tandem target-binding domains into a split aptamer (termed cooperative binding split aptamer, CBSA) to 
achieve more sensitive detection of cocaine, even in complex biological samples. The initial cocaine-binding event stabilizes the structure of the split aptamer and facilitates subsequent target-binding at the second binding domain. Our CBSA exhibits higher target affinity and far more responsive target-induced aptamer assembly compared to the single-domain parent split aptamer (PSA) from which it was derived. Using a fluorophore/quencher pair, we have demonstrated that a CBSA-based fluorescence assay can achieve sensitive and reproducible cocaine detection in biofluid specimens, with a LOD of $50 \mathrm{nM}$ in $10 \%$ saliva within $15 \mathrm{~min}$. Given the simplicity of splitting and engineering a CBSA from an isolated aptamer and the excellent performance of the CBSA-based assay in biofluid samples, it should be straightforward to develop other CBSA-based assays from existing or future aptamers for rapid, sensitive and specific detection of various targets in clinical or field settings in a simple, low-cost assay format.

\subsection{Experimental section}

\subsubsection{Materials}

2-amino-5,6,7-trimethyl-1,8-naphthyridine (ATMND) was purchased from Ryan Scientific and dissolved in dimethyl sulfoxide (DMSO) as a stock solution (50 $\mu \mathrm{M})$. Cocaine hydrochloride was purchased from Sigma-Aldrich. Benzoylecgonine tetrahydrate, (-)-nicotine, anhydroecgonine methyl ester and cocaethylene were purchased from Cerilliant Corporation and prepared as $50 \mathrm{mM}$ stock solutions in deionized water and stored at $4{ }^{\circ} \mathrm{C}$. Saliva samples were collected from eight healthy and drug-free volunteers (four males and four females) and centrifuged at 20,000 rcf for 15 min, with supernatants stored at $4^{\circ} \mathrm{C}$ before use. The pooled saliva matrices were prepared by mixing $1 \mathrm{~mL}$ of saliva supernatant from each donor. All DNA molecules 
were ordered from Integrated DNA Technologies and purified with HPLC and the sequences are listed in Table 3-1. DNA was dissolved in PCR grade water and DNA concentrations were measured with a NanoDrop 2000 (Thermo Scientific).

Table 3-1. Sequence ID and DNA sequences used in Chapter 3.

\begin{tabular}{ll}
\hline Sequence ID & Sequence (5' - 3') \\
\hline L-PSA & CTCCTTCAACGAAGTGGGTTCC \\
S-PSA & GG/iSpC3/ACAAGGAG \\
L-LSA & CTCCTTCAACGAAGTGGGTTCCTTGTCTC \\
L-5325 & CTCCTTCAACGAAGTGGGTTCCTTCAACGAAGTGGGTCTC \\
S-5325 & GAGACAAGG/iSpC3/ACAAGGAG3' \\
S-5325-Cy5 & /5IAbRQ/ GAGACAAGG/iSpC3/ACAAGGAGT/3Cy5Sp/ \\
L-CBSA-M1 & CTCCTTCAACGAAGTGGGTTCCTTCGACGAAGTGGGTCTC \\
L-CBSA-M2 & CTCCTTCGACGAAGTGGGTTCCTTCAACGAAGTGGGTCTC \\
L-5335 & CTCCTTCAACGAAGTGGGTCTCCTTCAACGAAGTGGGTCTC \\
S-5335 & GAGACAAGG/iSpC3/GACAAGGAG \\
S-5335-Cy5 & /5IAbRQ/GAGACAAGG/iSpC3/GACAAGGAGT/3Cy5Sp/ \\
L-5335-GT & CTCCTTCAATGAAGTGGGCTCCTTCAACGAAGTGGGTCTC \\
L-5435 & CTCCTTCAACGAAGTGGGTCTTCCTTCAACGAAGTGGGTCTC \\
S-5435 & GAGACAAGGA/iSpC3/GACAAGGAG \\
L-5445 & CTCCTTCAACGAAGTGGGTTCTTCCTTCAACGAAGTGGGTCTC \\
S-5445 & GAGACAAGGA/iSpC3/GAACAAGGAG \\
38-GC & GGGAGACAAGGAAAATCCTTCAACGAAGTGGGTCTCCC \\
38-GC-20A & GGGAGACAAGGAAAATCCTACAACGAAGTGGGTCTCCC \\
38-GC-20C & GGGAGACAAGGAAAATCCTCCAACGAAGTGGGTCTCCC \\
38-GC-21T & GGGAGACAAGGAAAATCCTTTAACGAAGTGGGTCTCCC \\
38-GC-22T & GGGAGACAAGGAAAATCCTTCTACGAAGTGGGTCTCCC \\
38-GC-22G & GGGAGACAAGGAAAATCCTTCGACGAAGTGGGTCTCCC \\
\hline /5IAbRQ/ represents Iowa Black RQ & b. /iSpC3/ represents internal C3 spacer \\
c. S-5325 served as short fragment for CBSA-5325, LSA, CBSA-M1 and CBSA-M2 \\
d. /3Cy5Sp/ represents Cy5 \\
\end{tabular}

\subsubsection{Characterization of split aptamer assembly using ATMND}

For each ATMND-binding assay, we mixed $10 \mu \mathrm{L}$ of $10 \times$ binding buffer $(100 \mathrm{mM}$ Tris, $1 \mathrm{mM} \mathrm{MgCl} 2, \mathrm{pH} 7.4), 85 \mu \mathrm{L}$ of deionized water, $1 \mu \mathrm{L}$ of each split aptamer fragment (final concentration $1 \mu \mathrm{M}$ ), $1 \mu \mathrm{L}$ ATMND solution (final concentration 200 
$\mathrm{nM}$ ) and $2 \mu \mathrm{L}$ of cocaine at different concentrations into wells of a 96-well plate. Fluorescence intensity was measured using Tecan M1000Pro with excitation at $358 \mathrm{~nm}$ and emission at $405 \mathrm{~nm}$ at a 2-minute time interval until the fluorescence intensity was stable. Each sample was analyzed in triplicate, and the means and standard deviations were plotted. Quenching was calculated by $\left(\mathrm{F}_{\mathrm{A}}-\mathrm{F}\right) / \mathrm{F}_{\mathrm{A}} \times 100 \%$, where $\mathrm{F}_{\mathrm{A}}$ is the fluorescence of $200 \mathrm{nM}$ ATMND in $1 \times$ binding buffer alone and $\mathrm{F}$ is the fluorescence of the ATMND-CBSA mixture with or without cocaine. Error bars show standard deviations obtained from three measurements.

\subsubsection{Isothermal titration calorimetry (ITC) experiments}

ITC experiments were performed with a MicroCal ITC200 (GE Healthcare). Cocaine and split aptamers were prepared with $1 \times$ binding buffer. The sample cell was initially loaded with $20 \mu \mathrm{M}$ of CBSA-5335 or PSA. $4 \mathrm{mM}$ of cocaine titrant was loaded into the syringe. Each experiment typically consisted of 39 successive $1 \mu \mathrm{L}$ injections after a 0.4 $\mu \mathrm{L}$ purge injection with spacing of 210 seconds to a final molar ratio of $43: 1$ (cocaine:aptamer). Split-aptamer experiments were performed at $20^{\circ} \mathrm{C}$. The raw data were first corrected based on the heat of dilution of cocaine, and then analyzed with the MicroCal analysis kit integrated into Origin 7 software. The titration curve of PSA was fitted with a single-site binding model and the titration curve of CBSA-5335 was fitted with independent-sites and cooperative-sites models with two binding sites.

\subsubsection{Fluorophore/quencher-modified CBSA-5325, -5335, or -5335-GT for detection of cocaine}

For each fluorophore/quencher-modified CBSA-based assay, we mixed $10 \mu \mathrm{L}$ of $10 \times$ binding buffer, $83 \mu \mathrm{L}$ of deionized water, $1 \mu \mathrm{L}$ of the CBSA long fragment and $1 \mu \mathrm{L}$ of 
the fluorophore/quencher-modified CBSA short fragment (final concentration $1 \mu \mathrm{M}$ each), and $5 \mu \mathrm{L}$ of cocaine at different concentrations into wells of a 96-well plate. We measured the fluorescence intensity with a Tecan M1000Pro with excitation at $648 \mathrm{~nm}$ and emission at $668 \mathrm{~nm}$ at room temperature after 15 minutes of incubation. Each sample was analyzed in triplicate, and the means and standard deviations were plotted. The data were fitted with the Hill equation using Origin 9 software to calculate Hill coefficient $\left(\mathrm{n}_{\mathrm{H}}\right)$ and cocaine concentration producing half occupancy $\left(\mathrm{K}_{1 / 2}\right)$. The signal gain was calculated by $\left(\mathrm{F}-\mathrm{F}_{0}\right) / \mathrm{F}_{0} \times 100 \%$, where $\mathrm{F}_{0}$ is the fluorescence of the CBSA mixture without cocaine and $\mathrm{F}$ is the fluorescence of CBSA mixtures with different concentrations of cocaine. Error bars show standard deviation of signal gains from three individual measurements at each cocaine concentration.

\subsubsection{Assessing saliva matrix and dilution effects on the CBSA-5335-based fluorophore/quencher assay}

Cocaine was first spiked into the pooled saliva (FIU IRB: IRB-13-0320) to create artificial samples with concentrations ranging from 0.25 to $500 \mu \mathrm{M}$. Each sample was prepared by mixing $10 \mu \mathrm{L}$ of $10 \times$ binding buffer, $1 \mu \mathrm{L}$ of CBSA long fragment and $1 \mu \mathrm{L}$ of fluorophore/quencher-modified CBSA short fragment (final concentration $1 \mu \mathrm{M}$ each) into wells of a 96 -well plate. 10 or $50 \mu \mathrm{L}$ of sample for each cocaine concentration was added into the well to analyze the $10 \%$ or $50 \%$ saliva matrices and deionized water was added to bring each well's volume to $100 \mu \mathrm{L}$. Fluorescence intensity with excitation at $648 \mathrm{~nm}$ and emission from 655-850 nm was scanned with a Tecan M1000Pro at room temperature after 15 minutes of incubation. Each sample was analyzed in triplicate, and 
the mean and standard deviation of the signal gain at different cocaine concentrations were plotted.

\subsubsection{Determining the sensitivity and dynamic range of CBSA-5335-based fluorophore/quencher assay in saliva}

For cocaine detection in $10 \%$ saliva, we combined $5 \mu \mathrm{L}$ of cocaine in solutions of concentrations ranging from 0.001 to $10 \mu \mathrm{M}$ with $10 \mu \mathrm{L}$ of pooled saliva, $10 \mu \mathrm{L}$ of $10 \times$ binding buffer, $1 \mu \mathrm{L}$ of CBSA long fragment, $1 \mu \mathrm{L}$ of fluorophore/quencher-modified CBSA short fragment (final concentration $1 \mu \mathrm{M}$ each), and $73 \mu \mathrm{L}$ of deionized water into wells of a 96-well plate. Fluorescence intensity with excitation at $648 \mathrm{~nm}$ and emission at $668 \mathrm{~nm}$ was measured with Tecan M1000Pro at room temperature after 15 minutes of incubation. Each sample was analyzed in triplicate, and the mean and standard deviation of the signal gain at different cocaine concentrations were plotted. A control calibration curve in buffer was also generated with cocaine concentrations ranging from 0.001 to 10 $\mu \mathrm{M}$ as described above. The signal gain was calculated by $\left(\mathrm{F}-\mathrm{F}_{0}\right) / \mathrm{F}_{0} \times 100 \%$, where $\mathrm{F}_{0}$ is the fluorescence without cocaine and $\mathrm{F}$ is the fluorescence with different concentrations of cocaine. Error bars show standard deviations from three measurements. The measurable LOD was determined using the lowest non-zero calibrator concentration according to the Scientific Working Group for Forensic Toxicology Standard Practices

for Method Validation in Forensic Toxicology. ${ }^{187}$ In all cases, the calibrators (cocaine standard concentration) were analyzed over three runs, and the lowest concentration achieving mean signal gain higher than 3.3 times the standard deviation was defined as the measurable LOD. 


\subsubsection{Determining the target specificity of CBSA-5335-based fluorophore/quencher assay in saliva}

The CBSA-5335 fluorophore/quencher assay was performed as described above with cocaine, cocaethylene, benzoylecgonine, anhydroecgonine methyl ester or nicotine at concentrations of 5 or $50 \mu \mathrm{M}$ in $10 \%$ saliva. Each sample was analyzed in triplicate and the mean and standard deviation of the signal gain at different cocaine concentrations were plotted. The cross-reactivity of each analyte at each concentration was calculated as a percentage based on $\operatorname{Sig}_{\mathrm{ANA}} / \operatorname{Sig}_{\mathrm{COC}} \times 100 \%$, where $\operatorname{Sig}_{\mathrm{ANA}}$ is the signal gain achieved by a given interferent and SigCOC is the signal gain achieved by cocaine at the same concentration.

\subsubsection{Determining the precision and bias of CBSA-5335-based}

\section{fluorophore/quencher assay in saliva}

The CBSA-5335 fluorophore/quencher assay was performed as described above in $10 \%$ diluted saliva matrices collected from eight different donors, $10 \%$ diluted pooled saliva matrices and binding buffer. We performed six measurements of samples containing final cocaine concentrations of $0,1,5$ and $10 \mu \mathrm{M}$, and plotted the mean and standard deviation of the signal gain at different cocaine concentrations. The bias of each cocaine concentration was calculated as $\left(\operatorname{Mean}_{\text {sam }}-\operatorname{Mean}_{\text {pool }}\right) / \operatorname{Mean}_{\text {pool }} \times 100 \%$, where Mean $_{\text {sam }}$ is the mean signal gain obtained in $10 \%$ saliva matrices collected from different donors, and Mean pool $_{\text {is }}$ the mean signal gain obtained in the $10 \%$ pooled saliva matrix. The precision within samples or between runs at different cocaine concentrations was calculated by performing a one-way ANOVA test with the measurement number (6) as the grouping variable. Within-sample precision at each cocaine level was calculated 
as $\sqrt{\mathrm{MS}_{\mathrm{wg}}} / \mathrm{Mean}_{\mathrm{sam}} \times 100 \%$, where $\mathrm{MS}_{\mathrm{wg}}$ is the within-group mean square obtained

from the ANOVA table, and Mean $_{\text {sam }}$ is the mean of signal gains obtained in $10 \%$ saliva matrices collected from different donors. Between-run precision at each cocaine level was calculated as $\sqrt{\frac{\mathrm{MS}_{\mathrm{bg}}+(n-1) \times \mathrm{MS}_{\mathrm{wg}}}{n}} / \mathrm{Mean}_{\text {sam }} \times 100 \%$, where $\mathrm{MS}_{\mathrm{bg}}$ is the betweengroup mean square obtained from the ANOVA table, and $\mathrm{n}$ is the total number of measurements $(n=6)$.

\subsection{Results and discussion}

\subsubsection{Design of the cocaine-binding CBSA}

A

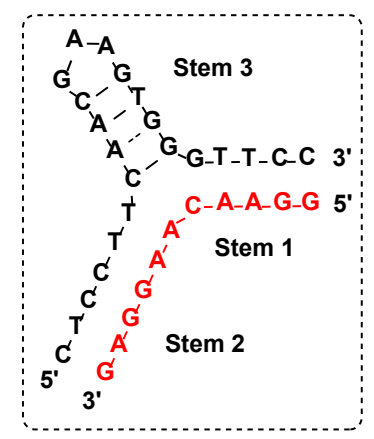

Split Cocaine-Binding Aptamers

\section{B}

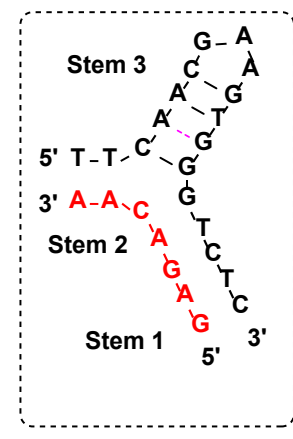

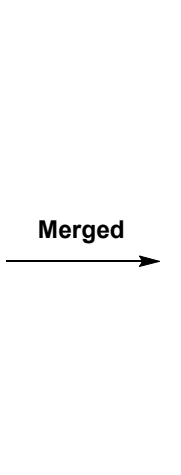

Cooperative Binding Split Aptamer (CBSA)

Figure 3-1. The design of our cocaine-binding CBSA. Two split aptamer pairs were derived from 38-GC. Stem 1 of one split aptamer (A) was merged with stem 2 of the other split aptamer (B) to form an engineered CBSA $(\mathbf{C})$ comprising a short fragment (SF) and a long fragment (LF).

Cooperative target binding of split aptamers requires the incorporation of at least two binding domains into a single pair of fragments. We previously reported a cocainebinding aptamer $(38-\mathrm{GC})^{102}$ that exhibits 2.5 -fold higher cocaine affinity than the MNS4.1 aptamer from which it was derived. 38-GC contains a three-way junction with the target-binding domain located at its center, surrounded by three double-stranded stems (stems 1, 2 and 3) and two loops (GAA and AAA loops). We have determined that stem 
3 is essential for cocaine binding, while both stem 1 and stem 2 contribute to the stability of the three-way junction structure that forms upon target binding. ${ }^{102}$ We derived two different pairs of split aptamer fragments from 38-GC (Figure 3-1, A and B), in which stem 3 remained intact but the 3 '-end of stems 1 and 2 were truncated and the AAA loop of stem 2 was eliminated. We subsequently produced a construct from these two sets of split aptamers, in which stem 1 from one set was linked to stem 2 from the second set (Figure 3-1C). The resulting CBSA consists of a short fragment (SF) and a long fragment (LF).

\subsubsection{Characterizing cocaine-induced CBSA assembly}

We anticipated that the CBSA fragments would remain separated in the absence of target, but would form two tandem cocaine-binding domains when fully assembled with cocaine. To confirm effective target-induced CBSA assembly, we developed a binding assay based on the fluorescent molecule ATMND. It has been reported that ATMND can strongly bind to a thymine situated opposite a C3 spacer abasic site (AP site) within a DNA duplex $\left(\mathrm{K}_{\mathrm{D}}=111 \mathrm{nM}\right)$ via three-point hydrogen bonding. ${ }^{188}$ Although ATMND fluoresces brightly when free in solution, this fluorescence is greatly quenched when ATMND is bound to a DNA duplex in this fashion. ${ }^{189}$ We therefore replaced the adenosine (at position 10 from 5') between the two binding domains of the short fragment with a C3 spacer to form an AP site with a thymine in the opposite position within the long fragment upon cocaine binding (Figure 3-2A). In the absence of cocaine, the long and short fragments remain separated, with strong fluorescence produced by the free ATMND molecules (Figure 3-2B, left). Upon addition of cocaine, the CBSA fragments undergo cooperative target-induced assembly and form a duplexed AP site that 
binds ATMND and quenches its fluorescence (Figure 3-2B, right). The resulting CBSA5325/cocaine complex contains four base-paired segments (Figure 3-2A, right; segments labeled A-D) and a dinucleotide bulge formed within each three-way junction. ${ }^{86}$

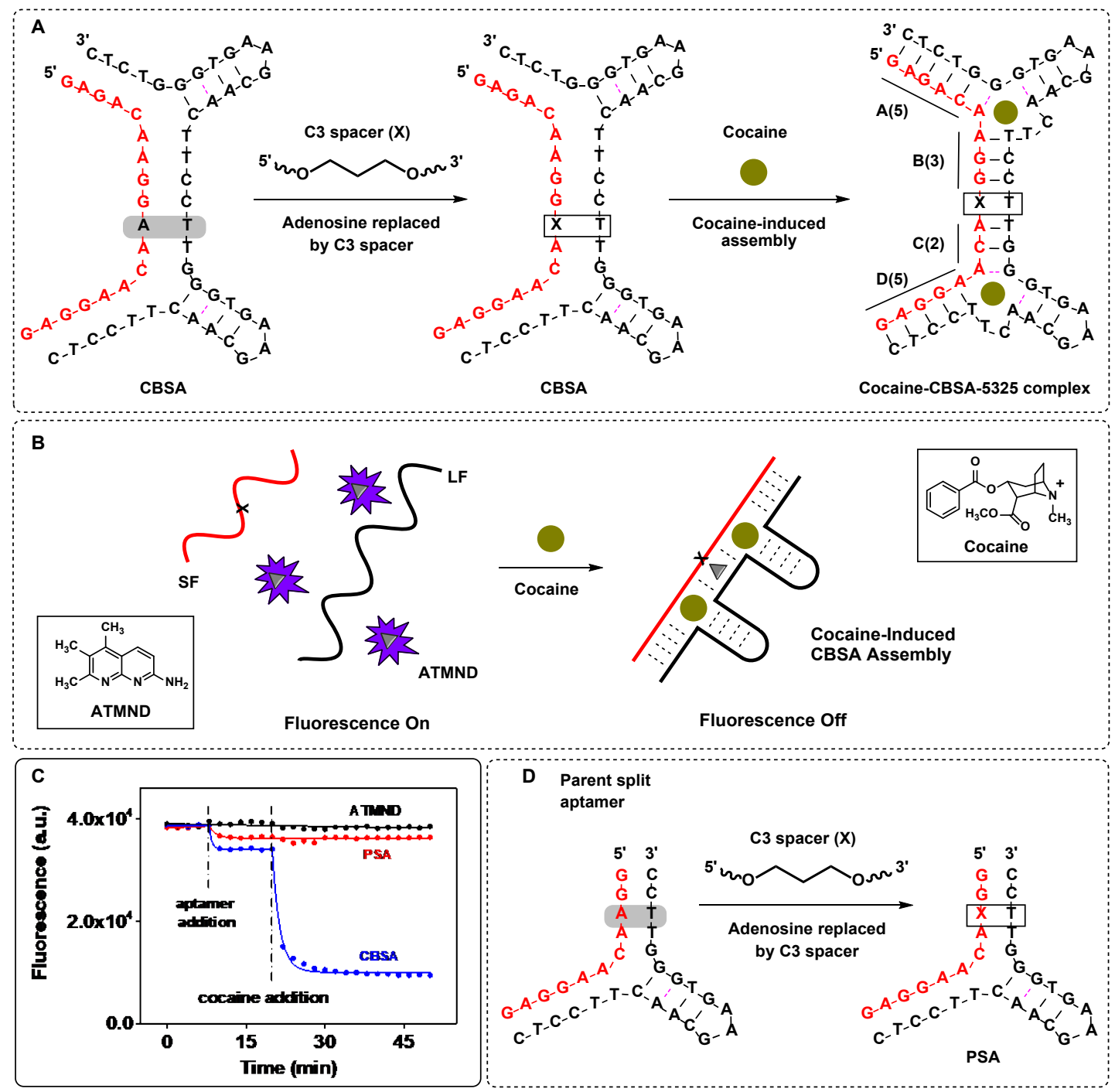

Figure 3-2. ATMND as a fluorescence reporter of target-induced CBSA assembly. (A) We modified the core CBSA sequence (left) by replacing the adenosine (at position 10 from 5') between the two binding domains of the short fragment with a $\mathrm{C} 3$ spacer abasic site (marked as $\mathrm{X}$ ) to yield the final CBSA construct (middle). Upon binding cocaine, the CBSA undergoes target-induced assembly, forming a dinucleotide bulge within each three-way junction (right). The cocaine-CBSA-5325 complex features four complementary double-stranded regions (A-D) and a duplexed AP site. (B) In the absence of cocaine, LF and SF remain separated and the unbound ATMND in solution generates a strong fluorescent signal. Cocaine induces CBSA assembly, forming the duplexed AP site that binds ATMND and thereby quenches its fluorescence. (C) Time-course of ATMND quenching by cocaine-induced split aptamer assembly. (D) Sequence of the parent split aptamer (PSA) with incorporated AP site. 


\subsubsection{Importance of dual-binding domain of CBSA for target-induced assembly}

We subsequently confirmed the cocaine-induced assembly of CBSA experimentally. When we mixed $1 \mu \mathrm{M}$ each of the long and short fragments with $200 \mathrm{nM}$ ATMND in $1 \times$ binding buffer (10 mM Tris-HCl, $100 \mu \mathrm{M} \mathrm{MgCl}_{2}, \mathrm{pH} 7.4$ ), we observed 10\% background quenching (Figure 3-2C, before cocaine addition). This quenching is most likely attributable to low levels of non-specific quench and non-target assembly of the CBSA. Upon addition of $250 \mu \mathrm{M}$ cocaine, $72 \%$ of the ATMND fluorescence was quenched within 15 min, indicating rapid target-induced CBSA assembly (Figure 3-2C, after cocaine addition). We also tested the performance of the AP-incorporating parent split aptamer (PSA) from which the CBSA was initially derived (Figure 3-2D, PSA), which features only a single cocaine-binding domain, in our ATMND-binding assay. In the absence of cocaine, the PSA quenched $6 \%$ of ATMND fluorescence (Figure 3-2C, before cocaine addition), and no measureable signal change was observed upon addition of $250 \mu \mathrm{M}$ cocaine (Figure 3-2C, after cocaine addition). This indicates that no cocaineinduced assembly is taking place, presumably because the shortening of stem 1 to four base-pairs with an abasic site in the middle results in poor thermodynamic stability of the cocaine-PSA complex at room temperature. We therefore conclude that the quenching observed most likely arises through nonspecific interactions between PSA and ATMND.

Aptamer binding affinity ${ }^{78,190}$ and DNA hybridization efficiency ${ }^{191}$ are both strongly affected by magnesium concentration. We therefore used our ATMND-binding assay to optimize cocaine-induced CBSA assembly by varying the $\mathrm{Mg}^{2+}$ concentration from 0 to $1000 \mu \mathrm{M}$, and observed that maximum cocaine-induced ATMND quenching occurred in the presence of $100 \mu \mathrm{M} \mathrm{Mg}^{2+}$ (Figure 3-3A). In the absence of cocaine, we observed 9\% 
quenching without $\mathrm{Mg}^{2+}$, which we attributed to nonspecific CBSA assembly (Figure 3-3A, no cocaine). The presence of cocaine only generated an additional $7 \%$ quenching due to the absence of the $\mathrm{Mg}^{2+}$ counterion, resulting in strong repulsion between the CBSA fragments (Figure 3-3A, cocaine). At high concentration of $\mathrm{Mg}^{2+}(1000 \mu \mathrm{M})$, extensive CBSA assembly occurred in the absence of cocaine, producing $38 \%$ ATMND quenching, while also reducing the binding affinity of CBSA to cocaine, which only produced an additional 17\% quenching upon addition of $250 \mu \mathrm{M}$ cocaine (Figure 3-3A).

A

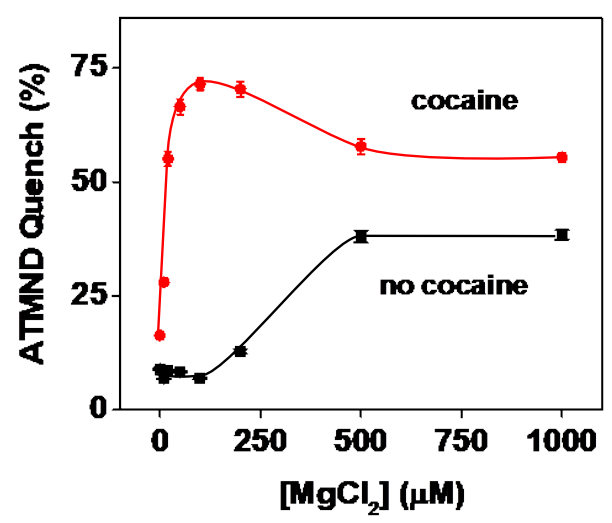

B

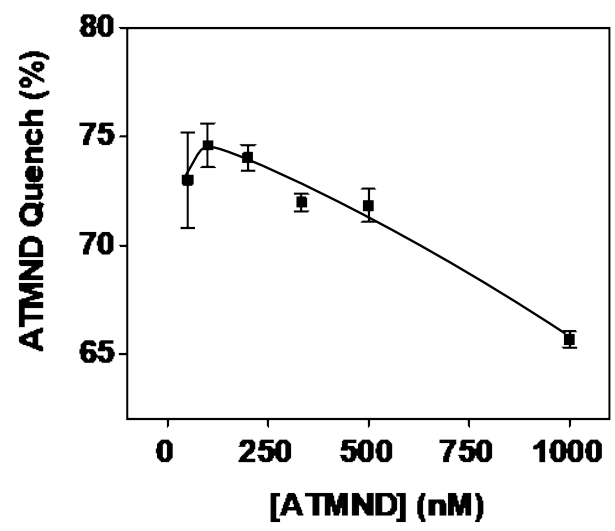

Figure 3-3. Optimization of $\mathrm{Mg}^{2+}$ and ATMND concentrations for our CBSA-based ATMNDbinding assay. (A) ATMND (200 $\mathrm{nM})$ quenching by target-induced CBSA assembly upon addition of $250 \mu \mathrm{M}$ cocaine in the presence of $0-1000 \mu \mathrm{M} \mathrm{Mg}^{2+}$ in $1 \times$ binding buffer. Quenching was calculated by $\left(\mathrm{F}_{\mathrm{A}}-\mathrm{F}\right) / \mathrm{F}_{\mathrm{A}} \times 100 \%$, where $\mathrm{F}_{\mathrm{A}}$ is the fluorescence of $200 \mathrm{nM}$ ATMND in $1 \times$ binding buffer alone and $\mathrm{F}$ is the fluorescence of the ATMND-CBSA mixture with $250 \mu \mathrm{M}$ cocaine (red curve) or without cocaine (black curve), respectively. (B) Quenching analysis with ATMND concentrations ranging from $50-1000 \mathrm{nM}$ in $1 \times$ binding buffer containing $100 \mu \mathrm{M} \mathrm{Mg}^{2+}$. Quenching was calculated by $\left(\mathrm{F}_{0}-\mathrm{F}\right) / \mathrm{F}_{0} \times 100 \%$, where $\mathrm{F}_{0}$ and $\mathrm{F}$ are the fluorescence of the ATMND-CBSA in the absence and presence of cocaine, respectively.

We also varied the ATMND concentration from 50 to $1000 \mathrm{nM}$, and found that 200 nM ATMND produced low background and high target-induced signal change (Figure 3-3B). Under these optimized conditions (200 nM ATMND and $100 \mu \mathrm{M} \mathrm{Mg}^{2+}$ ), we examined the extent of target-induced CBSA assembly at different cocaine concentrations. We found a strong correlation between ATMND quenching from CBSA 
assembly and cocaine concentration in the range of 0 to $250 \mu \mathrm{M}$ (Figure 3-4). We characterized the binding affinity of the assembled-CBSA for ATMND by titrating different concentrations $(0-20 \mu \mathrm{M})$ of CBSA into $200 \mathrm{nM}$ ATMND in the presence of 1 $\mathrm{mM}$ cocaine (Figure 3-5). The calculated $\mathrm{K}_{\mathrm{D}}$ was $365 \mathrm{nM}$, which is consistent with the reported value for ATMND binding to AP sites. ${ }^{188}$ We subsequently demonstrated that the presence of two binding sites makes CBSA-5325 far more responsive to the presence of cocaine than split aptamers containing a single binding domain. We produced a long split aptamer (LSA) from CBSA, in which we replaced one of the binding domains with fully complementary sequences (Figure 3-6A, LSA). The LSA fragments quenched 75\% of ATMND fluorescence in the absence of cocaine. Addition of $250 \mu \mathrm{M}$ cocaine only induced an additional 10\% signal change, indicating that most LSA fragments were stably pre-assembled even without target (Figure 3-6B). In contrast, CBSA-5325 generated a large signal change $(72 \%)$ upon addition of $250 \mu \mathrm{M}$ cocaine because of its dual target binding domains, with far weaker background signal (9\%) without cocaine.

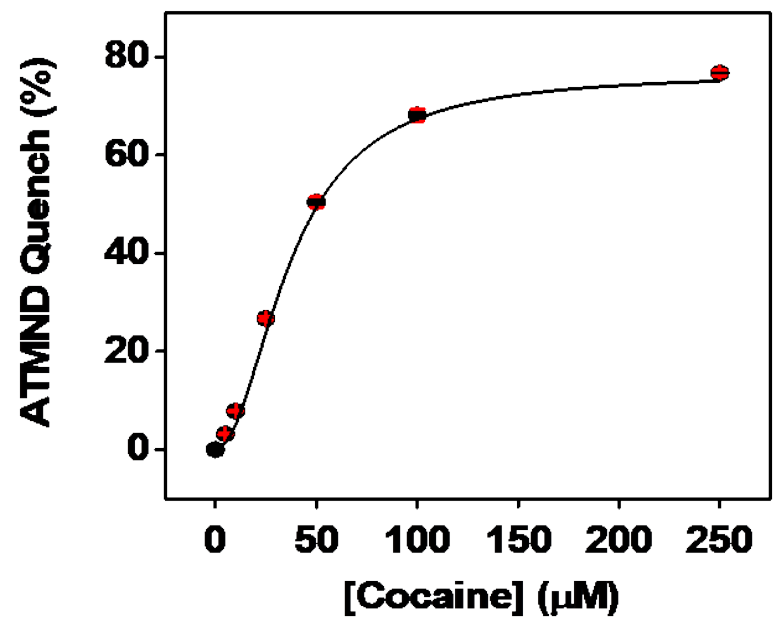

Figure 3-4. ATMND-reported calibration curve for CBSA-5325 at varying cocaine concentrations. Error bars show standard deviations from three measurements. Quenching was calculated by $\left(\mathrm{F}_{0}-\mathrm{F}\right) / \mathrm{F}_{0} \times 100 \%$, where $\mathrm{F}_{0}$ and $\mathrm{F}$ are the fluorescence of the ATMND-CBSA in the absence and presence of cocaine, respectively. 


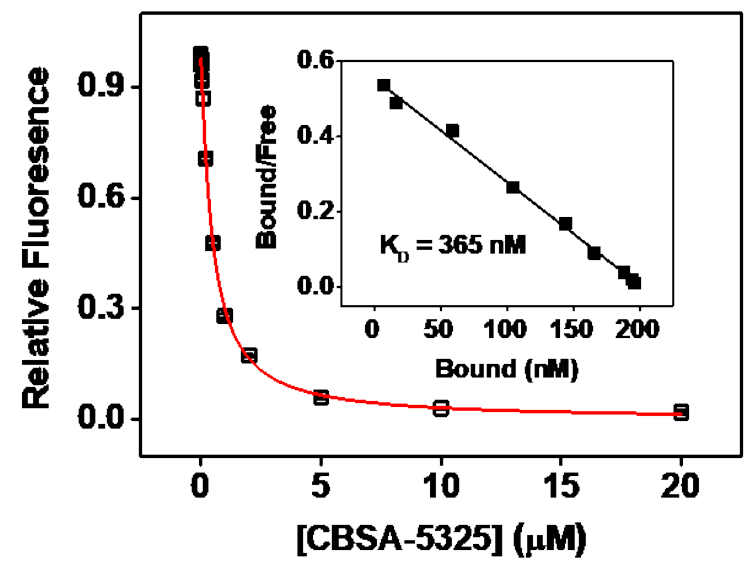

Figure 3-5. ATMND binding affinity for cocaine-assembled CBSA-5325. ATMND (200 nM) fluorescence was quenched with increasing concentrations of CBSA-5325 in the presence of 1 $\mathrm{mM}$ cocaine. Inset: Scatchard plot of the fluorescence data. The $\mathrm{K}_{\mathrm{D}}$ was determined based on the negative reciprocal of the slope.

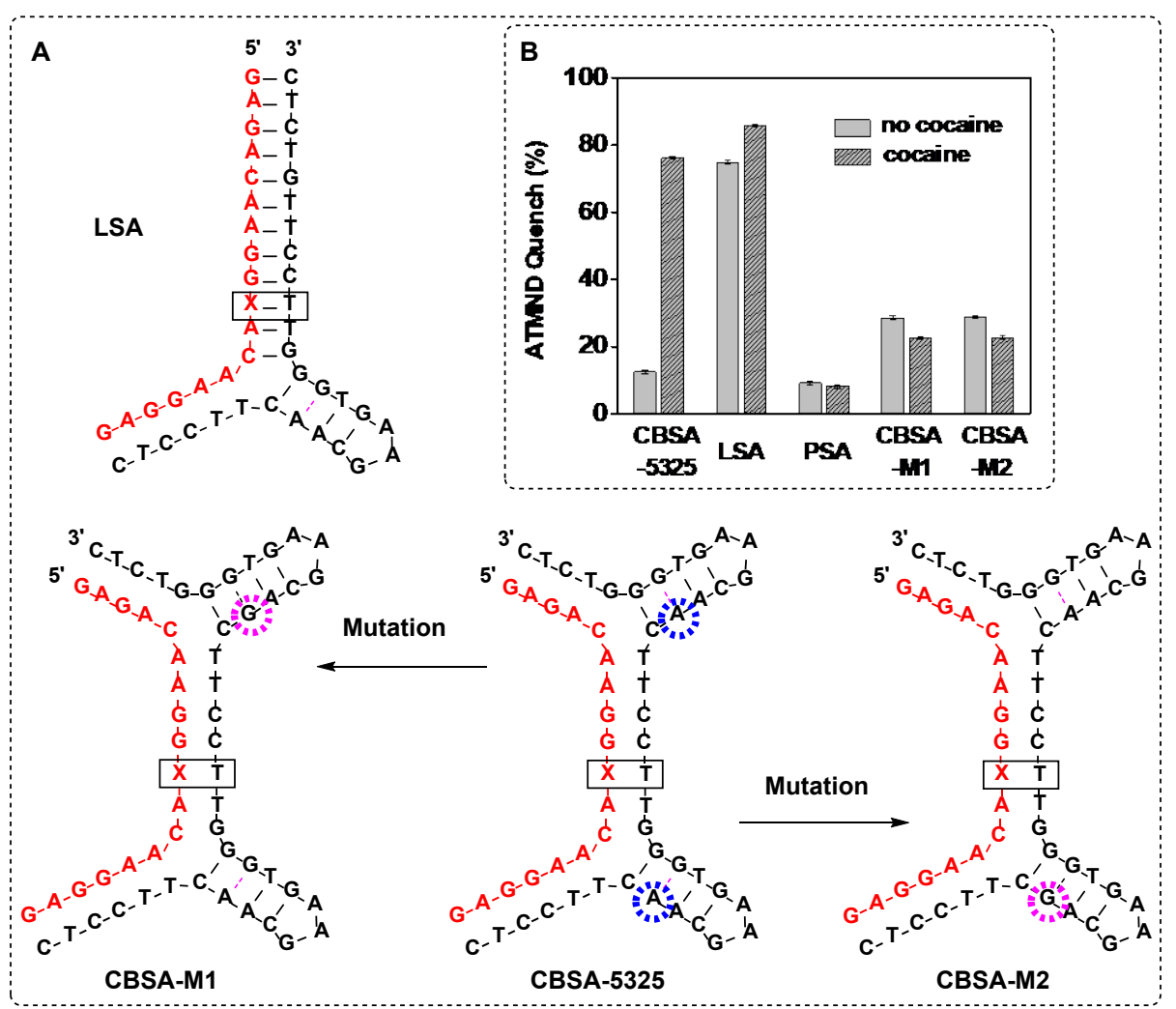

Figure 3-6. Analyzing the dual binding domains of CBSA for target-induced assembly. (A) We generated multiple derivatives of CBSA-5325, including split aptamers with a single binding pocket (LSA) and a pair of point-mutants (CBSA-M1 and CBSA-M2) with sequence alterations that disrupt either of the two binding domains (pink circle). (B) ATMND quenching in the presence or absence of $250 \mu \mathrm{M}$ cocaine. Quenching was calculated by $\left(\mathrm{F}_{\mathrm{A}}-\mathrm{F}\right) / \mathrm{F}_{\mathrm{A}} \times 100 \%$, where $\mathrm{F}_{\mathrm{A}}$ is the fluorescence of $200 \mathrm{nM}$ ATMND in $1 \times$ binding buffer alone and $\mathrm{F}$ is the fluorescence of the ATMND-CBSA mixture with $250 \mu \mathrm{M}$ cocaine or without cocaine, respectively. 


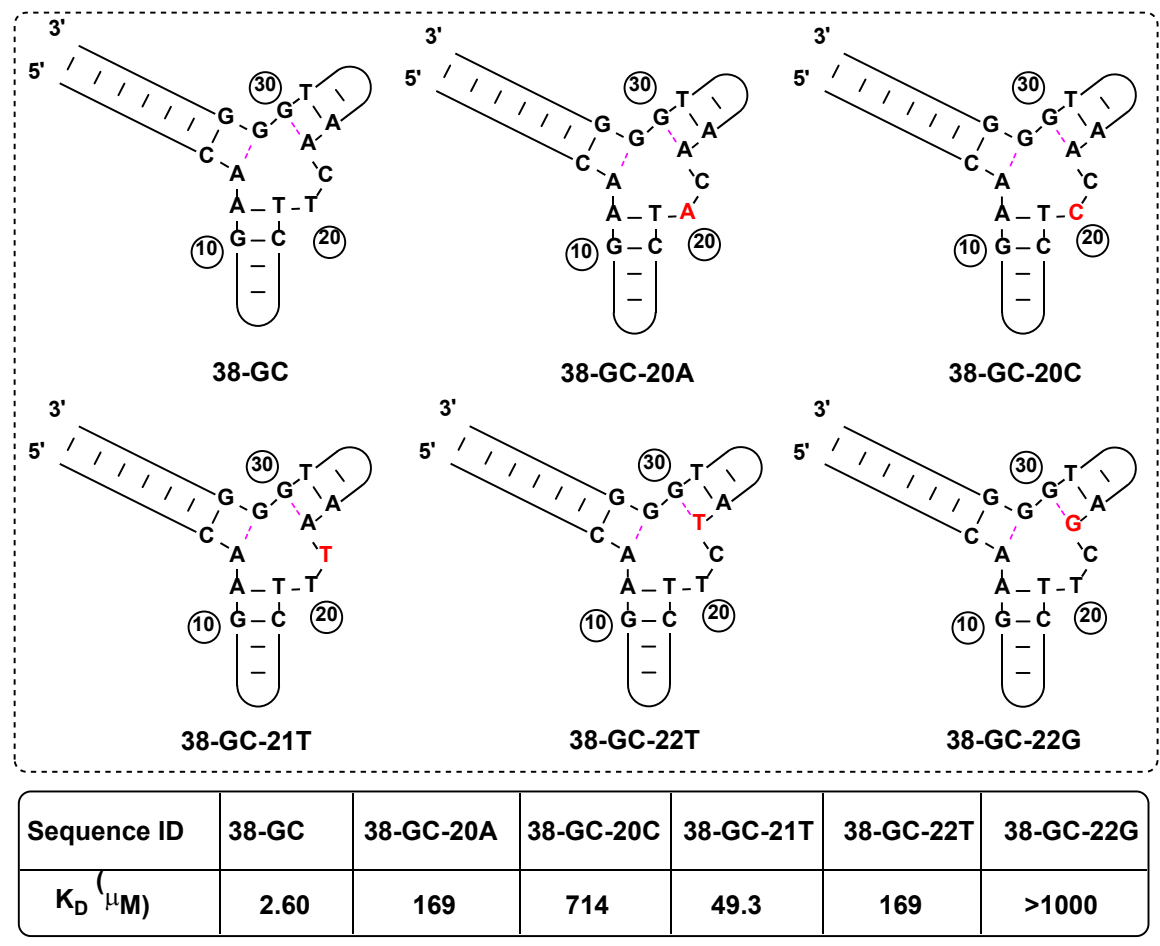

Figure 3-7. $\mathrm{K}_{\mathrm{D}}$ of $38-\mathrm{GC}$ and different $38-\mathrm{GC}$ mutants for cocaine as characterized by ITC. The sample cell was initially loaded with $20 \mu \mathrm{M}$ of aptamers. $500 \mu \mathrm{M}$ of cocaine titrant was loaded into the syringe. Each experiment typically consisted of 19 successive $2 \mu \mathrm{L}$ injections after a 0.4 $\mu \mathrm{L}$ purge injection with spacing of 150 seconds to a final molar ratio of 5.5:1 (cocaine:aptamer). The experiments were performed at $25^{\circ} \mathrm{C}$. The raw data were first corrected based on the heat of dilution of cocaine, and then analyzed with the MicroCal analysis kit integrated into Origin 7 software with a single-site binding model.

Specific target binding at both domains is required for target-CBSA assembly. We tested the binding affinity of $38-\mathrm{GC}$ and several point-mutated derivatives using ITC (Figure 3-7). We found that replacing an adenosine at position 22 with guanine completely impaired cocaine binding (Figure 3-7, 38-GC-22G). Based on this finding, we created two CBSA mutants (CBSA-M1 and CBSA-M2) in which either of the two binding domains was disrupted by this single-nucleotide mutation, leaving only a single binding domain capable of binding cocaine (Figure 3-6A). CBSA-M1 and CBSA-M2 were mutated at the 3'- and 5'-binding domain of the long fragment, respectively. We tested these mutants using the same ATMND-binding assay, and found that neither 
CBSA-M1 nor CBSA-M2 was capable of cocaine-induced aptamer assembly, with no significant ATMND quenching observed upon addition of cocaine (Figure 3-6B). These results confirmed that both target-binding domains of the CBSA were required for targetinduced aptamer assembly, and thus provide strong support for a cooperative-bindingbased assembly mechanism.

\subsubsection{Confirmation of cooperative target binding of CBSA and fabricating an ultra-sensitive CBSA-based fluorophore/quencher assay}

We hypothesized that the binding affinity of the CBSA might be enhanced by further stabilizing the target/aptamer complex with additional base-pairs, based on prior findings that longer complementary stems surrounding the three-way junction increased the aptamer's target-binding affinity. ${ }^{87}$ Thus, we increased the total number of base-pairs between the two binding domains of CBSA-5325 by adding an additional one, two or three base-pairs to generate CBSA-5335, -5435 and -5445 (Figure 3-8A) and examined the extent of target-induced CBSA assembly at different cocaine concentrations $(0-50$ $\mu \mathrm{M})$. Our results demonstrated that the number of additional base-pairs greatly affects both binding affinity and target-induced assembly. In the absence of cocaine, the quenching of ATMND fluorescence increased as the number of base-pairs between the two CBSA binding domains increased from 5- to 8-bp (Figure 3-8B). This is probably due to the increased thermo-stability of CBSA assembly, even without target. Upon addition of cocaine, we found a strong correlation between ATMND quenching from CBSA assembly and cocaine concentration in the range of 0 to $50 \mu \mathrm{M}$ (Figure 3-8C). Compared to CBSA-5325, quenching saturation occurred at lower target concentrations for the other CBSAs, in keeping with the assumption that the CBSA binding affinity can 
be enhanced with additional base-pairs. However, considerable background assembly was observed for CBSA-5435 and CBSA-5445 in the absence of cocaine, reducing their target-induced signal gain. We thus found that CBSA-5335 is most responsive and exhibited the most extensive target-induced CBSA assembly, and we therefore used this construct for subsequent sensor development.

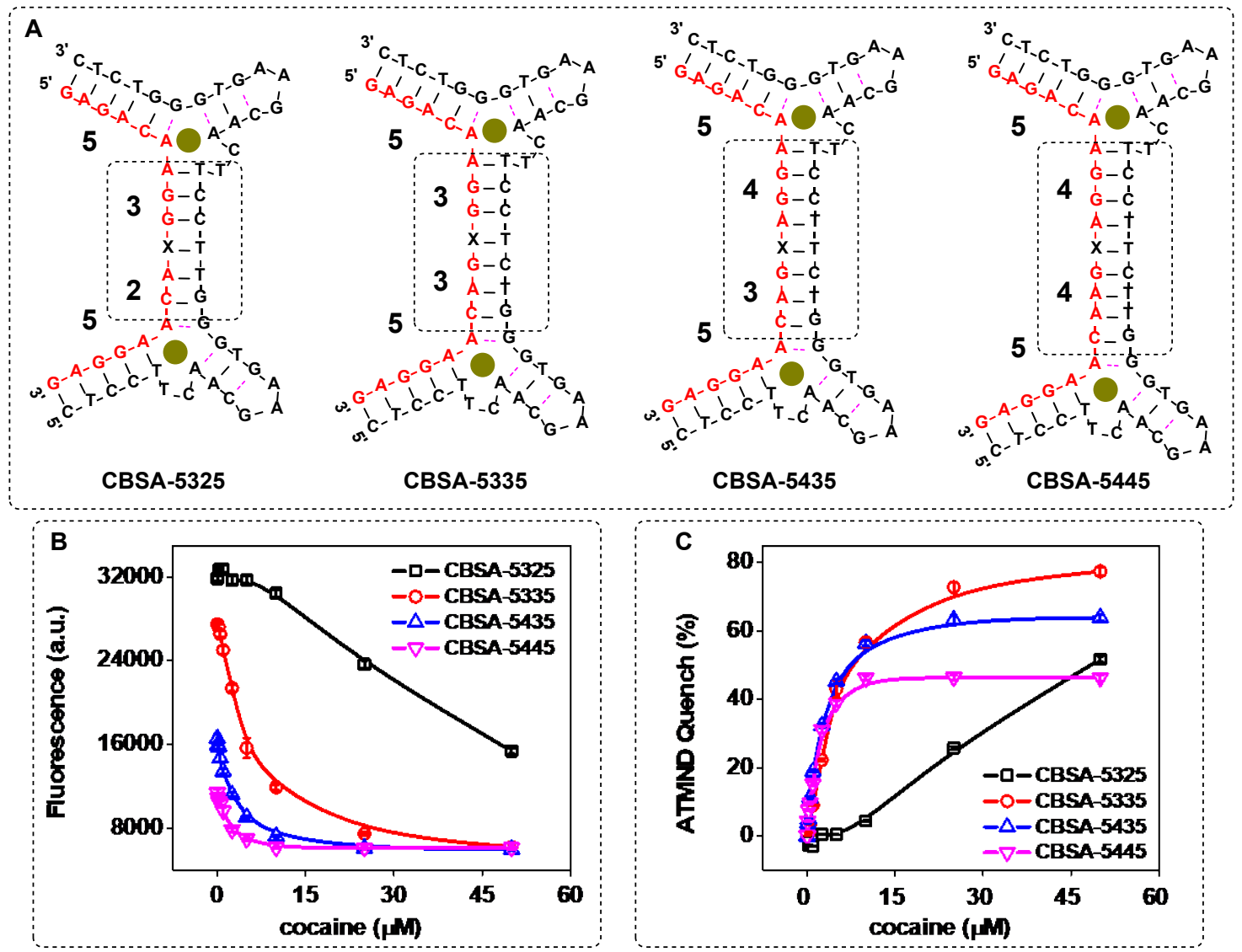

Figure 3-8. Stem length effects on cocaine-induced CBSA assembly. (A) Sequences of CBSA$5325,-5335,-5435$ and -5445 . (B) ATMND (200 nM) fluorescence upon addition of $1 \mu \mathrm{M}$ CBSA-5325, $-5335,-5435$ or -5445 at various cocaine concentrations $(0-50 \mu \mathrm{M})$. (C) ATMNDreported calibration curve for these CBSA variants at varying cocaine concentrations. Quenching was calculated by $\left(\mathrm{F}_{0}-\mathrm{F}\right) / \mathrm{F}_{0} \times 100 \%$, where $\mathrm{F}_{0}$ and $\mathrm{F}$ are the fluorescence of the ATMND-CBSA in the absence and presence of cocaine, respectively. 

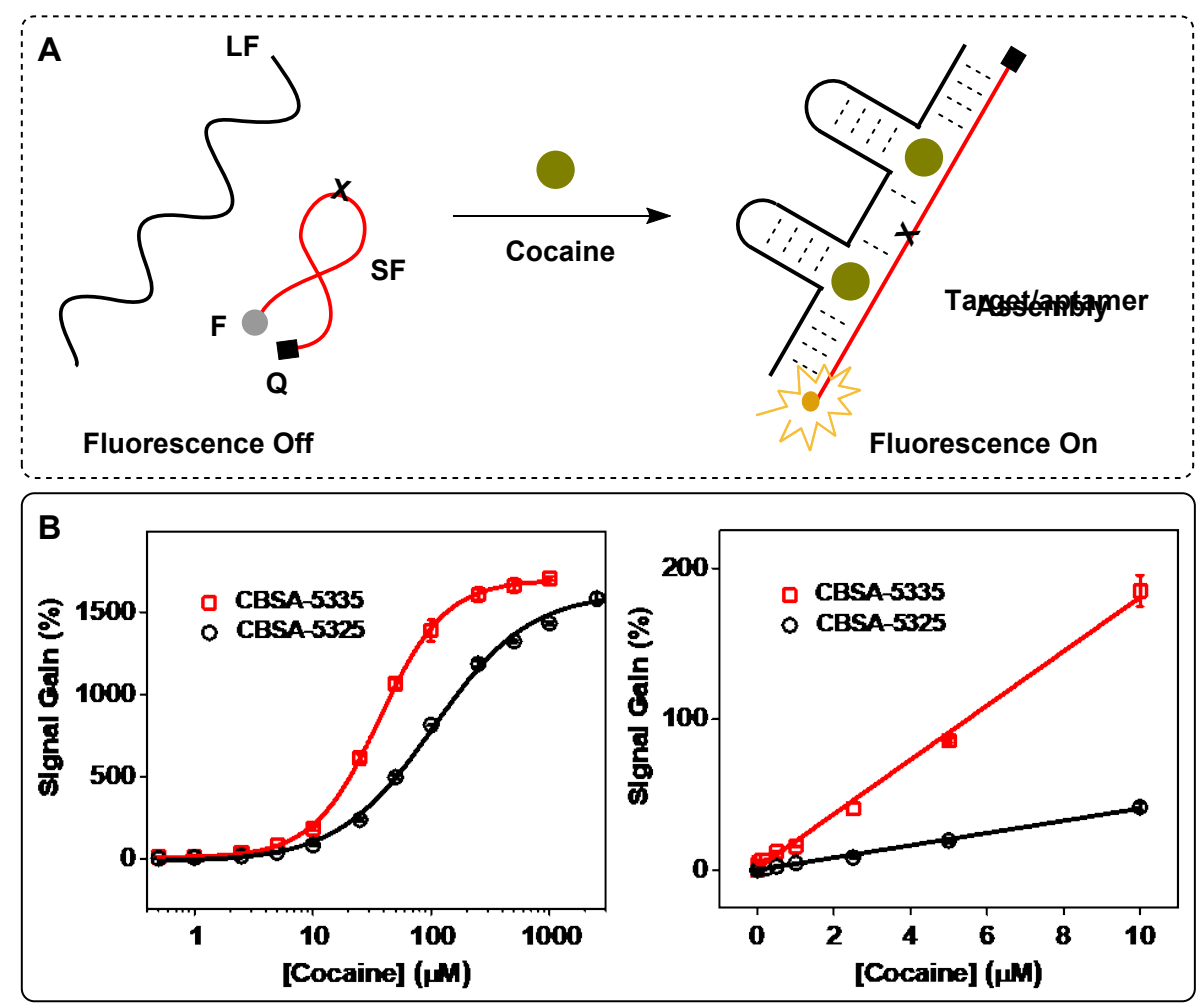

Figure 3-9. Effect of target-binding affinity $\left(\mathrm{K}_{1 / 2}\right)$ on CBSA cooperativity. (A) The working principle of the CBSA-based fluorophore/quencher assay. (B) Calibration curves for the assay at cocaine concentrations ranging from 0 to $2500 \mu \mathrm{M}$ (left). Right panel shows a linear response at $0-10 \mu \mathrm{M}$. Reactions were performed with $1 \mu \mathrm{M}$ CBSA long fragment, $1 \mu \mathrm{M}$ fluorophore/quencher-modified CBSA short fragment, and different concentrations of cocaine in $10 \mathrm{mM}$ Tris- $\mathrm{HCl}, 0.1 \mathrm{mM} \mathrm{MgCl} 2(\mathrm{pH} 7.4)$ at room temperature.

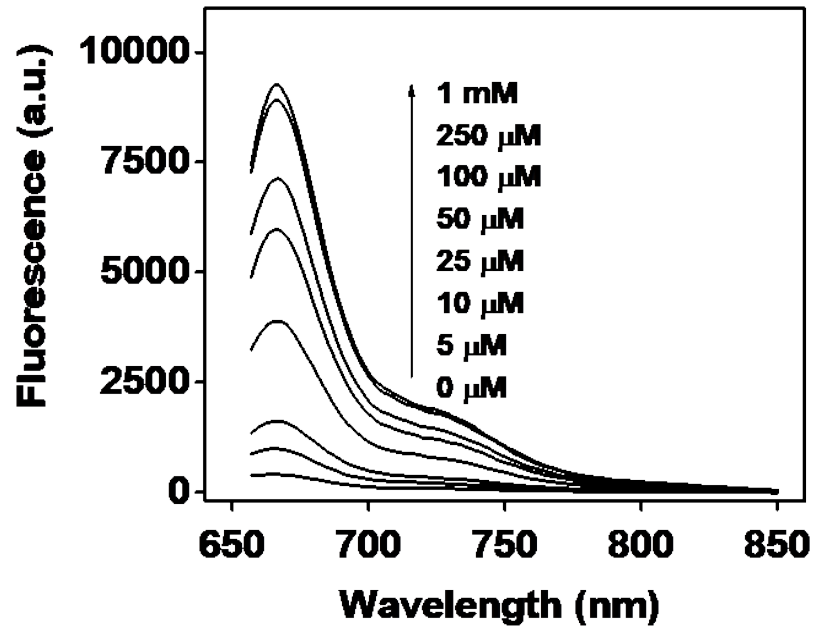

Figure 3-10. Spectra of CBSA-5335-based fluorescent detection of cocaine in $1 \times$ binding buffer. In the absence of cocaine, we observed negligible emission from 655 to $850 \mathrm{~nm}$ when excited at $648 \mathrm{~nm}$. The fluorescence peak at $668 \mathrm{~nm}$ increased with increasing cocaine concentration. 
We then produced fluorophore/quencher-modified derivatives of CBSA-5325 and CBSA-5335 to achieve sensitive detection of cocaine. The short fragment was modified with an IowaBlack RQ quencher at its 5' terminus and a Cy5 fluorophore at its 3' terminus. In the absence of cocaine, the two CBSA fragments remain separate, and the flexibility of the unbound short fragment routinely brings the fluorophore into close proximity with the quencher, resulting in very low fluorescence (Figure 3-9A, left). In the presence of cocaine, the two fragments assemble to form a rigid target/aptamer structure that separates the fluorophore/quencher pair, producing increased fluorescence (Figure 3-9A, right). We used these two fluorophore/quencher-modified CBSAs to generate calibration curves for cocaine concentrations ranging from 0-1000 $\mu \mathrm{M}$ (Figure 3-9B; spectra of CBSA-5335 shown in Figure 3-10) and used the Hill equation (Equation 3-1) to fit the binding curve to calculate $\mathrm{K}_{1 / 2}$ and Hill coefficient $\left(\mathrm{n}_{\mathrm{H}}\right):^{186,192}$

$$
\text { Binding site occupancy } \bar{Y}=\frac{[\text { Target }]^{n_{H}}}{[\text { Target }]^{n_{H}+\left(K_{1 / 2}\right)^{n_{H}}}}
$$

(Equation 3-1)

We determined a $\mathrm{K}_{1 / 2}$ of $106 \mu \mathrm{M}$ with an $\mathrm{n}_{\mathrm{H}}$ of 1.1 for CBSA-5325 and a $\mathrm{K}_{1 / 2}$ of 36 $\mu \mathrm{M}$ with an $\mathrm{n}_{\mathrm{H}}$ of 1.5 for CBSA-5335 (where $\mathrm{K}_{1 / 2}$ represents the cocaine concentration at which half of the binding domains are occupied and $n_{H}$ describes the order of binding cooperativity). ${ }^{192}$ An $n_{H}$ of 1.5 clearly indicates higher cooperativity between the two binding domains of CBSA-5335. We found that the measurable LOD was $500 \mathrm{nM}(4.5 \pm$ $0.8 \%)$ and $50 \mathrm{nM}(4.3 \pm 0.9 \%)$ for CBSA-5325 and CBSA-5335, respectively (Figure 3-9B). Clearly, the target-binding affinity of CBSA can be affected by the cooperativity of the two binding domains. Note that CBSA-5335 has the highest cocaine affinity of any split aptamer described to date, and the LOD of CBSA-5335 is more than 200-fold lower 
than that of a previously-described single-domain, split aptamer-based cocaine assay using a similar sensing platform $(\mathrm{LOD}=10 \mu \mathrm{M}){ }^{21}$

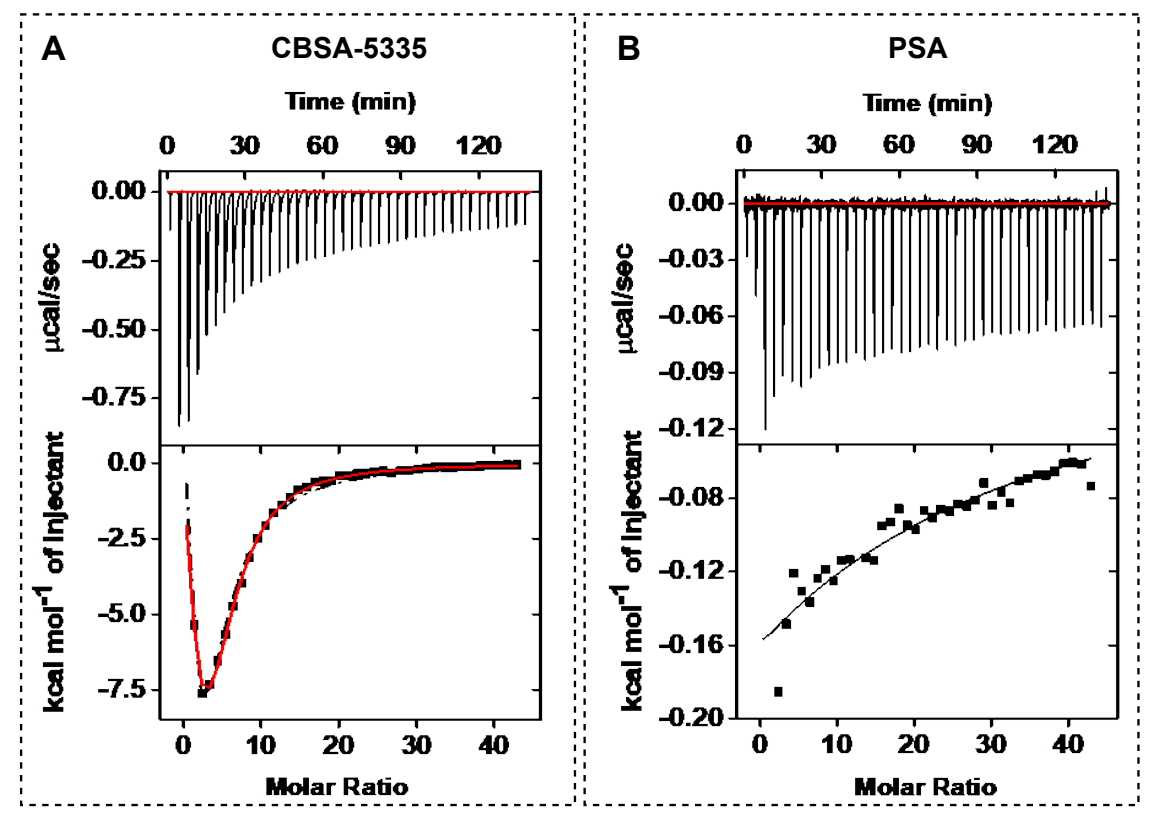

Figure 3-11. Characterization of cocaine binding affinity of CBSA-5335 and PSA using ITC. Top panels present raw data showing the heat generated from each titration of cocaine for (A) CBSA-5335 (black broken line represented independent-sites model and red solid line represented cooperative-sites model) and (B) PSA. Bottom panels show the integrated heat of each titration after correcting for dilution heat of the titrant.

We subsequently used ITC to characterize the binding mechanism and affinity of CBSA-5335 for its target at both sites. Recognizing that the $\mathrm{K}_{\mathrm{D}}$ of the aptamers was in the $\mu \mathrm{M}$ range, we set up a titration with a high cocaine:aptamer ratio to obtain accurate $\mathrm{K}_{\mathrm{D}}$ values for each binding scenario (the adjacent binding pocket empty, or occupied by cocaine), where $K_{D}$ represents the ligand concentration at which half of the receptor sites are occupied at equilibrium. ${ }^{193}$ The resulting two-phase titration curve confirmed the interaction of cocaine with the two binding domains of CBSA-5335 (Figure 3-11A). The binding stoichiometry between cocaine and CBSA was manually set as two, because previous studies have demonstrated that one cocaine-binding aptamer binds a single molecule of cocaine. ${ }^{102}$ ITC data of CBSA-5335 were then fitted with both independent- 
sites and cooperative-sites models ${ }^{194}$ with two binding sites (Figure 3-11A, black broken line represented independent-sites model and red solid line represented cooperative-sites model). We observed better fitting using the cooperative-sites model, and determined that the $\mathrm{K}_{\mathrm{D}}$ for the initial and secondary cocaine-binding events were 116 and $36 \mu \mathrm{M}$, respectively (Figure 3-11A). Based on these $\mathrm{K}_{\mathrm{D}}$, we calculated a $\mathrm{K}_{1 / 2}$ of $65 \mu \mathrm{M}$ and a $\mathrm{n}_{\mathrm{H}}$ of 1.3 for CBSA-5335, ${ }^{195}$ which is comparable with the results obtained via the CBSA5335-based fluorophore/quencher assay $\left(\mathrm{K}_{1 / 2}=36 \mu \mathrm{M}\right.$ and $\left.\mathrm{n}_{\mathrm{H}}=1.5\right)$. Notably, the low $\mathrm{K}_{1 / 2}$ of CBSA-5335 represents a 56 -fold higher target binding affinity relative to its single-domain PSA ( $\mathrm{K}_{\mathrm{D}}=2 \mathrm{mM}$, Figure 3-11B).

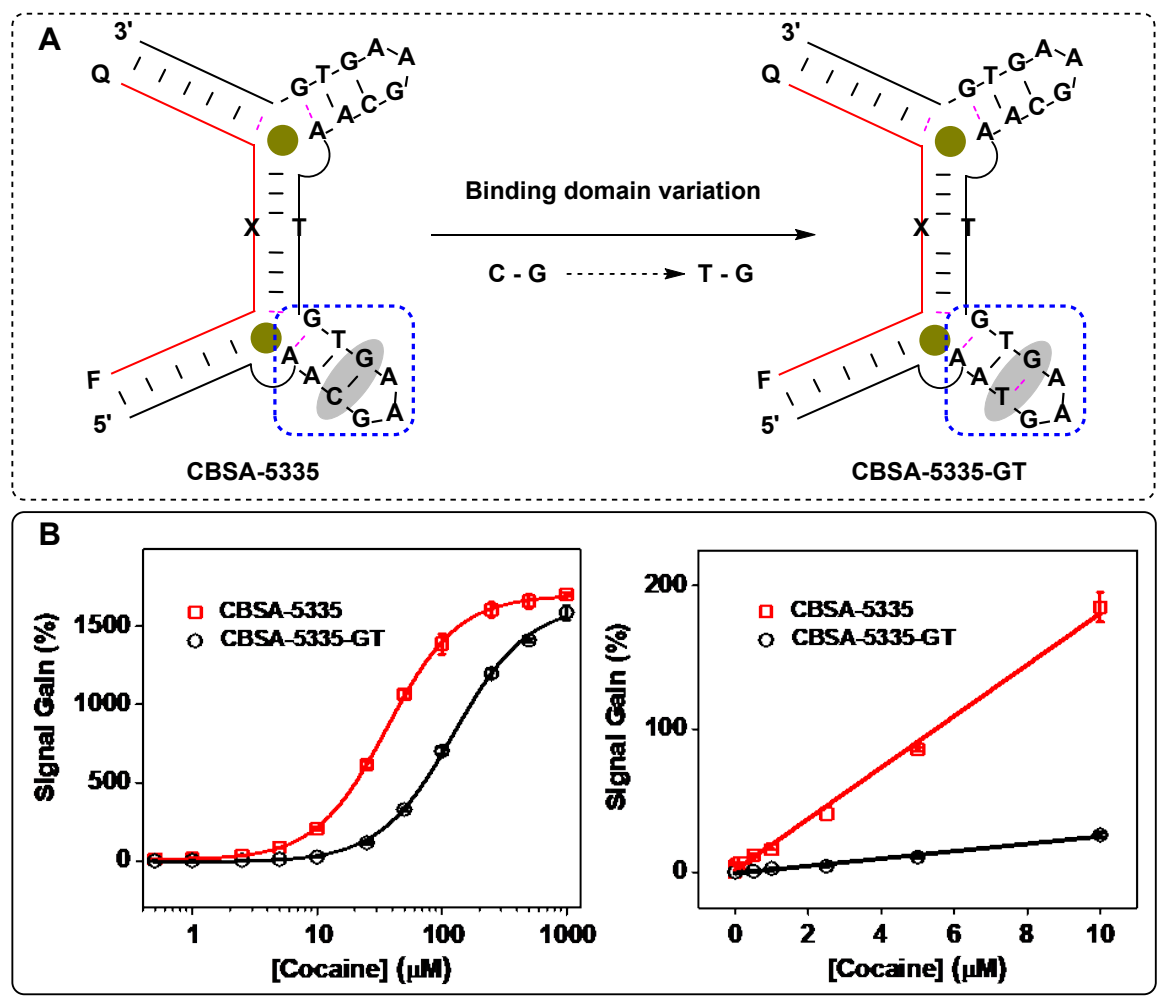

Figure 3-12. Effect of the intrinsic affinity of the PSA on CBSA target-binding affinity $\left(\mathrm{K}_{1 / 2}\right)$. (A) We generated CBSA-5335-GT by replacing a G-C base-pair (highlighted) in the 5' binding domain of CBSA-5335 with a G-T wobble pair. (B) Calibration curves for the assay at cocaine concentrations ranging from 0 to $1000 \mu \mathrm{M}$ after a 15-minunte incubation (left), with a linear response at $0-10 \mu \mathrm{M}$ (right). Experiments were performed with $1 \mu \mathrm{M}$ CBSA long fragment, $1 \mu \mathrm{M}$ fluorophore/quencher-modified CBSA short fragment, and different concentrations of cocaine in $10 \mathrm{mM}$ Tris- $\mathrm{HCl}, 0.1 \mathrm{mM} \mathrm{MgCl} 2(\mathrm{pH} 7.4)$ at room temperature. 
We believed that the target-binding affinity of CBSA could be affected by the intrinsic binding affinity of its parent split aptamer. To address this point, we replaced a G-C pair in the 5' binding domain of CBSA-5335 with a wobble G-T pair to form CBSA-5335GT. This alteration in stem 3 of the cocaine-binding aptamer reduces its binding affinity to cocaine ${ }^{102}$ (Figure 3-12A), but we expected the resulting CBSA to still retain its cooperativity. Indeed, our fluorescence results demonstrated that both CBSAs have identical cooperativity $\left(\mathrm{n}_{\mathrm{H}}=1.5\right)$ (Figure 3-12B). However, since the intrinsic affinity of 38-GC is 4-fold higher than the parent split aptamer variant used for CBSA-5335-GT, CBSA-5335 yields a lower $\mathrm{K}_{1 / 2}(33 \mu \mathrm{M})$ and better sensitivity (LOD $=50 \mathrm{nM}$ in buffer) than CBSA-5335-GT $\left(\mathrm{K}_{1 / 2}=125 \mu \mathrm{M}\right.$ and $\mathrm{LOD}=500 \mathrm{nM}$ in buffer) (Figure 3-12B).

\subsubsection{Validation of the CBSA-5335-based fluorophore/quencher assay for cocaine detection in saliva samples}

We subsequently confirmed that our CBSA-5335-based fluorophore/quencher assay is capable of equally sensitive cocaine detection in saliva samples. The excitation wavelength for Cy5 (648 $\mathrm{nm}$ ) does not induce auto-fluorescence in the saliva matrix, and thus produces minimal background fluorescence (Figure 3-13). Additionally, the high quenching efficiency of the IowaBlack RQ quencher ${ }^{196}$ allowed robust detection of cocaine at very low concentrations. To ensure that our CBSA-5335-based fluorophore/quencher assay can be reliably used in real-world (i.e., clinical or field) settings, we evaluated the assay's performance according to Scientific Working Group for Forensic Toxicology Standard Practices. ${ }^{187}$ To this end, we performed detailed experiments to investigate matrix effects, reaction time, limit of detection, interference effects and bias and precision in saliva samples. 


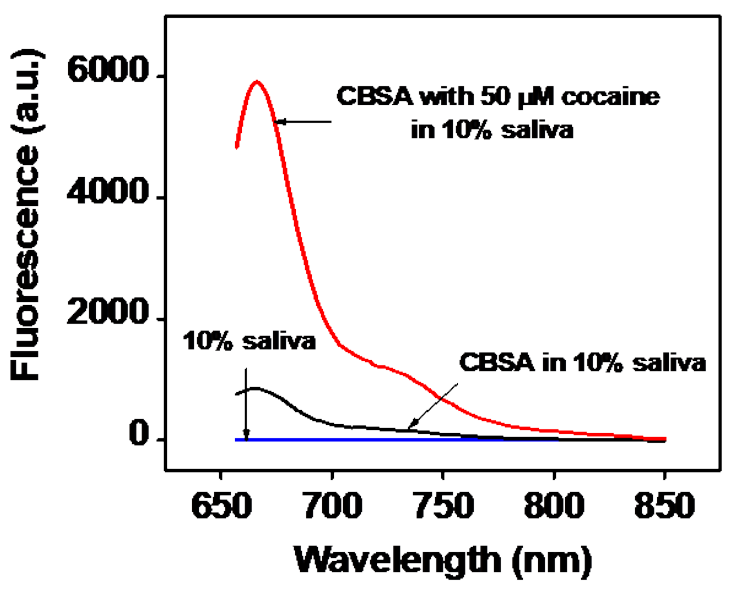

Figure 3-13. Fluorescent detection of cocaine in saliva with our fluorophore/quencher modified CBSA-5335. 10\% saliva (blue) exhibited no emission from 655 to $850 \mathrm{~nm}$ when excited at 648 $\mathrm{nm}$. CBSA-5335 alone (black) yielded only slight fluorescence background when excited at 648 $\mathrm{nm}$, but we observed a significant increase in fluorescence at $668 \mathrm{~nm}$ with the addition of $50 \mu \mathrm{M}$ cocaine (red).

A

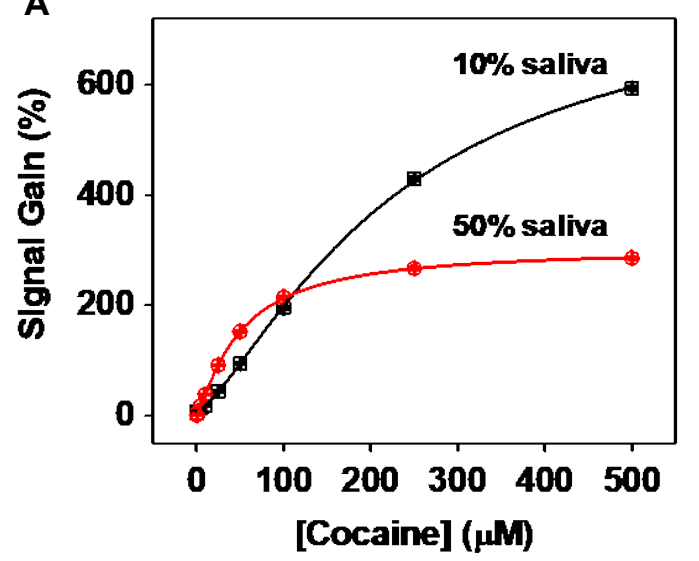

B

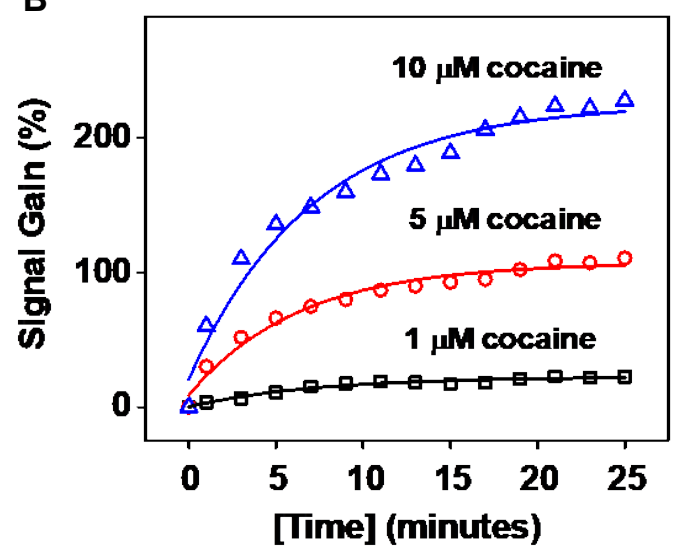

Figure 3-14. Successful detection of cocaine in saliva samples with fluorophore/quenchermodified CBSA-5335. (A) Dilution effects on cocaine detection in saliva. Saliva samples spiked with cocaine were tested with CBSA-5335 at $50 \%$ or $10 \%$ dilutions. Calibration curves were constructed based on signal gain at each concentration of cocaine in the pre-dilution sampler. (B) Time course of CBSA-5335 in the presence of 1,5 and $10 \mu \mathrm{M}$ cocaine in $10 \%$ pooled samples.

To test the matrix effects on assay performance, we mixed eight different saliva samples collected from healthy and drug-free donors of diverse gender and ethnic backgrounds as a pooled matrix. The pooled matrix was then spiked with different concentrations of cocaine ( 0 to $500 \mu \mathrm{M})$ and diluted with binding buffer $1: 1(50 \%)$ or 1:9 (10\%) before being applied to the CBSA-5335-based fluorophore/quencher assay. Our 
results showed that the $10 \%$ dilution resulted in a higher signal gain with a broader dynamic range $(0-100 \mu \mathrm{M}$ in the initial saliva sample) compared to the $50 \%$ dilution ( 0 $25 \mu \mathrm{M}$ in the initial sample) (Figure 3-14A). Additionally, the 10\% dilution compensates for variations (such as salt concentration and $\mathrm{pH}$ ) in individual saliva samples, and we therefore used $10 \%$ dilutions for subsequent experiments.
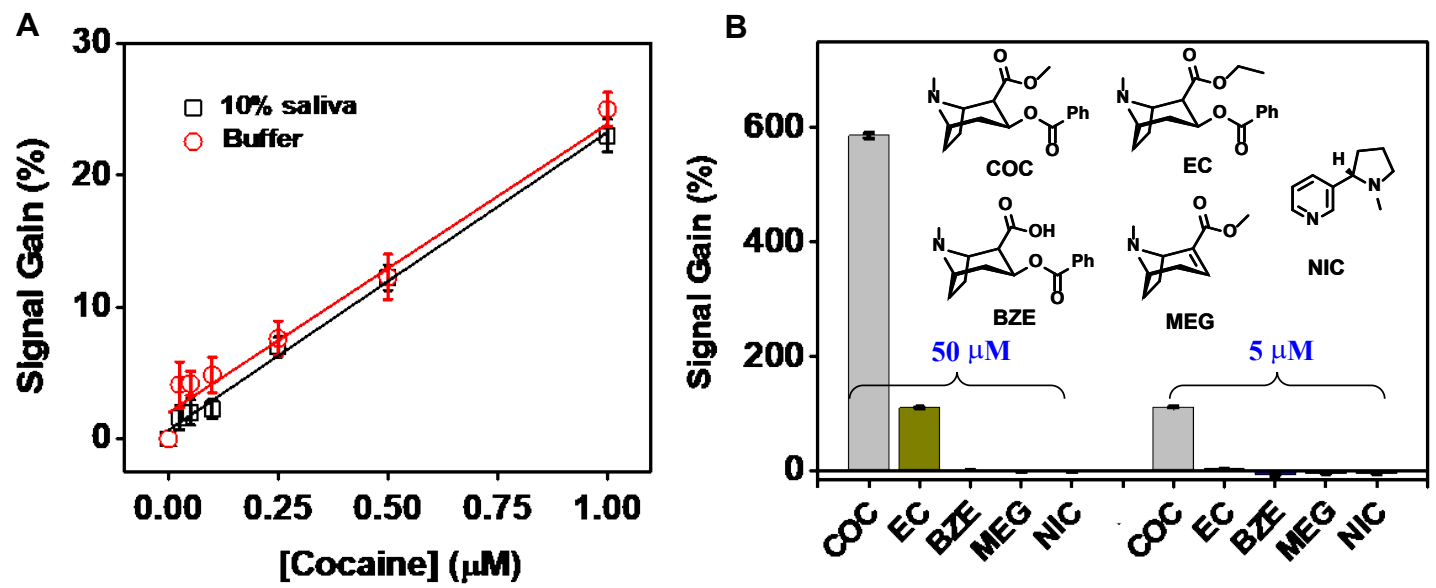

Figure 3-15. High sensitivity and specificity of our CBSA-5335-based fluorophore/quencher assay for cocaine detection in saliva. (A) Calibration curve for the assay in buffer and $10 \%$ saliva. (B) Signal gains from the CBSA assay in the presence of $50 \mu \mathrm{M}$ (left) and $5 \mu \mathrm{M}$ (right) cocaine (COC) or potential interferents including cocaethylene (EC), benzoylecgonine (BZE), anhydroecgonine methyl ester (MEG) and nicotine (NIC) in 10\% saliva. Inset shows the interferent structures. Error bars show standard deviation of signal gain from three measurements at each concentration.

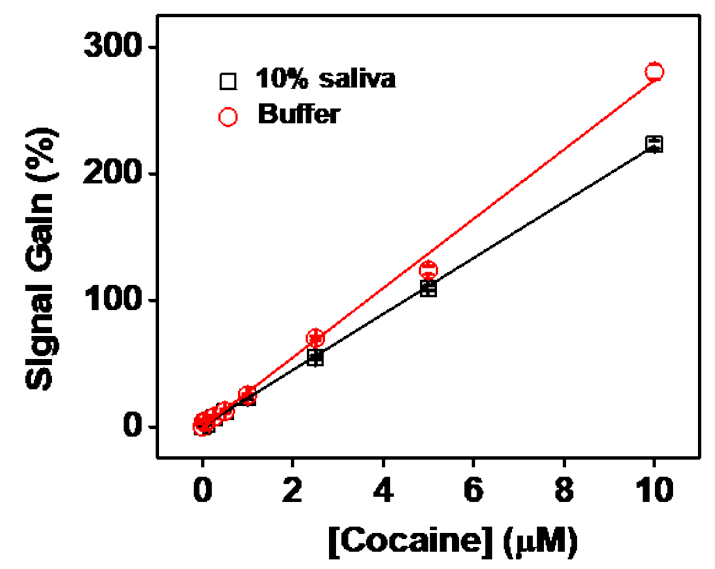

Figure 3-16. Calibration curve of our CBSA-5335-based fluorophore/quencher assay in $1 \times$ binding buffer and $10 \%$ saliva at cocaine concentrations ranging from 0 to $10 \mu \mathrm{M}$. The signal gain was calculated by $\left(\mathrm{F}-\mathrm{F}_{0}\right) / \mathrm{F}_{0} \times 100 \%$, where $\mathrm{F}_{0}$ is the fluorescence without cocaine and $\mathrm{F}$ is the fluorescence with different concentrations of cocaine. Error bars show standard deviation of signal gain from three measurements at each concentration. 
We also monitored the time course of our CBSA-5335-based fluorophore/quencher assay in $10 \%$ pooled saliva matrix. We found that the fluorescence signal greatly increased with the increase of reaction time upon the addition of 1,5 or $10 \mu \mathrm{M}$ cocaine and $85 \%$ of the maximum signal was obtained after 15 minutes (Figure 3-14B). Clearly, the fast reaction time of our assay is suitable for on-site detection due to the rapid assembly of cocaine-CBSA complex in saliva samples.

CBSA-5335-based fluorophore/quencher assay also demonstrates excellent performance on target detection in complex samples. Studies have shown that cocaine concentrations are generally higher in saliva than serum within the first few hours of administration, ${ }^{197,198}$ and the European Union's Driving Under the Influence of Drugs, Alcohol and Medicines (DRUID) program identified $510 \mathrm{nM}$ as the recommended cut-off sensitivity for road-side screening of cocaine in undiluted saliva. ${ }^{197}$ To determine the sensitivity of our assay, we generated a calibration curve in $10 \%$ saliva samples, obtaining a linear range from 0 to $10 \mu \mathrm{M}$ and a measurable LOD of $50 \mathrm{nM}$ (signal gain $3.3 \pm 0.8 \%$, Figure 3-15A and Figure 3-16). This suggests that, accounting for the ten-fold sample dilution, our assay can meet the recommendations established by DRUID for onsite detection of cocaine, with a detectable LOD equivalent to $500 \mathrm{nM}$ in undiluted saliva. In contrast, split aptamers containing a single binding domain have previously achieved LODs of $30 \mathrm{nM}$ in $0.5 \%$ saliva, ${ }^{199} 5 \mu \mathrm{M}^{200}$ and $3.8 \mu \mathrm{M}^{201}$ in $25 \%$ saliva. Two factors contribute to the high sensitivity of our CBSA assay. First, the low thermo-stability of the split aptamer greatly suppresses non-specific assembly of the CBSA fragments. Second, the cooperative binding from the two target-binding domains significantly increases the 
CBSA's affinity, resulting in a 56-fold reduction in $\mathrm{K}_{\mathrm{D}}(36 \mu \mathrm{M})$ relative to its singledomain PSA (2 mM).

We then investigated the specificity of the CBSA-5335-based fluorophore/quencher assay for cocaine versus structurally-similar and -dissimilar interferents in saliva. Benzoylecgonine (BZE), anhydroecgonine methyl ester (MEG), and cocaethylene (EC) are major structurally-similar metabolites of cocaine that are secreted into oral fluids. ${ }^{202}$ We tested our CBSA-5335-based assay with high concentrations of these metabolites as well as nicotine (NIC), since tobacco is widely used among cocaine users. We found that our CBSA assay showed excellent cocaine specificity: our results demonstrated no measurable signal from $50 \mu \mathrm{M}$ of BZE, MEG, or NIC and only $19 \%$ and $3 \%$ crossreactivity to $50 \mu \mathrm{M}$ and $5 \mu \mathrm{M} \mathrm{EC}$, respectively, in $10 \%$ saliva (Figure 3-15B). The results are consistent with previously reported assays ${ }^{21,93}$ based on single-domain cocainebinding split aptamers, demonstrating that CBSA retains excellent specificity for its target molecule in saliva. Finally, we tested the bias and precision of our CBSA assay by spiking cocaine at low, medium, and high concentrations into $10 \%$ saliva samples (final concentration of 1,5 or $10 \mu \mathrm{M}$ ) from eight different individuals (Figure 3-17A). Using the pooled saliva as a standard, the average bias of signal gain obtained in these individual samples was $12.7 \%,-0.4 \%$ and $-5.8 \%$ for 1,5 and $10 \mu \mathrm{M}$ cocaine, respectively. At 1,5 and $10 \mu \mathrm{M}$, the coefficients of variation (CV) within samples were $7.1 \%, 5.2 \%$ and $9.0 \%$, respectively, and the $\mathrm{CV}$ between runs were $7.3 \%, 5.1 \%$ and $8.5 \%$, respectively (Figure $3-17 \mathrm{~B}$ ). Thus, the bias and $\mathrm{CV}$ were consistently below the acceptable cut-off $(20 \%)$ for drug-screening methods, ${ }^{187}$ further demonstrating the immediate feasibility of our CBSA-based assay for on-site drug screening. 


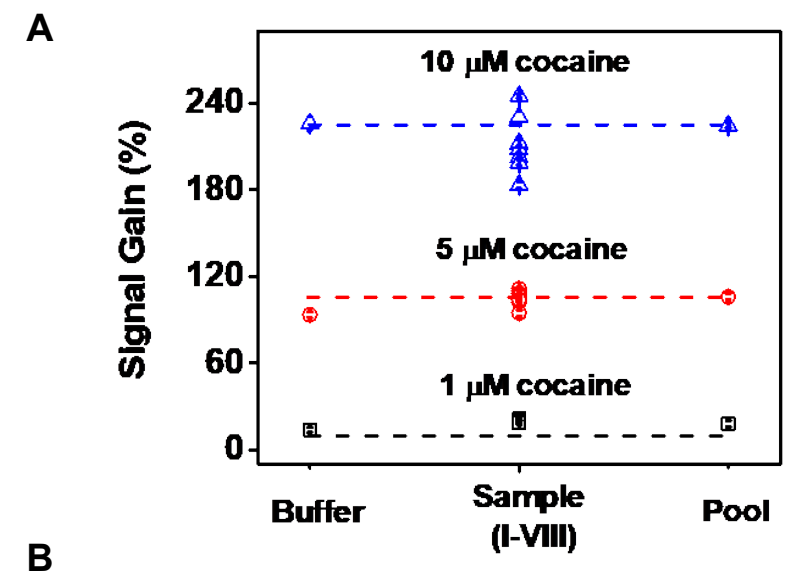

\begin{tabular}{|c|c|c|c|c|c|}
\hline Cocaine & \multicolumn{2}{|c|}{ Signal gain (\%) } & Bias & \multicolumn{2}{c|}{ Precision } \\
\hline $\begin{array}{c}\text { Conc. } \\
\left.\mu_{M}\right)\end{array}$ & $\begin{array}{c}\text { Mean } \\
(\text { samples) }\end{array}$ & $\begin{array}{c}\text { Mean } \\
(\text { pool) }\end{array}$ & $\begin{array}{c}\text { Bias } \\
(\%)\end{array}$ & $\begin{array}{c}\text { CV (\%) } \\
\text { within-sample }\end{array}$ & $\begin{array}{c}\text { CV (\%) } \\
\text { between-run }\end{array}$ \\
\hline 1 & 20 & 18 & 12.7 & 7.1 & 7.3 \\
\hline 5 & 105 & 106 & -0.4 & 5.2 & 5.1 \\
\hline 10 & 212 & 225 & -5.8 & 9.0 & 8.5 \\
\hline
\end{tabular}

Figure 3-17. Bias and precision of the CBSA-5335-based fluorophore/quencher assay. (A) Signal gain obtained from various cocaine concentrations in buffer, $10 \%$ saliva collected from different donors (Sample I-VIII) and $10 \%$ pooled saliva. Signal gain was calculated by $\left(\mathrm{F}-\mathrm{F}_{0}\right) / \mathrm{F}_{0} \times 100 \%$, where $F_{0}$ is the fluorescence of the CBSA without cocaine and $F$ is the fluorescence of the CBSA with cocaine. Error bars show standard deviations from six measurements. (B) Bias at each cocaine concentration was calculated from the mean signal gain obtained with samples I-VIII and the pooled sample. Within-sample and between-run precision at each cocaine concentration was calculated by one-way ANOVA.

\subsection{Conclusions}

We have demonstrated greatly improved split-aptamer-based sensing of cocaine in both buffer and saliva with a CBSA that incorporates two tandem target-binding domains into a single split aptamer. The CBSA fragments remain separate in the absence of target but specifically assemble upon addition of cocaine, and show high sensitivity to target concentration as a result of increased binding affinity caused by cooperative binding behavior. We achieved rapid, one-step detection of cocaine by simply mixing our CBSA components, which consist of a fluorophore/quencher-modified short fragment and an 
unmodified long fragment, with a cocaine-containing sample at room temperature. A measurable LOD of $50 \mathrm{nM}$ in buffer was achieved within 15 minutes without any signal amplification. The sensor's performance was also very robust in saliva, achieving a LOD of $50 \mathrm{nM}$ cocaine in $10 \%$ diluted saliva. Our assay also demonstrates very high target specificity, as evidenced by acceptably low cross-reactivity to common interferents, strong reproducibility, as well as a cost of $\$ 1.28$ per test, confirming the potential value of our CBSA-based assay for on-site detection of cocaine.

Our CBSA-based assay can be generalized in terms of both targets and sensor platforms. Stojanovic et al. recently isolated new aptamers for four different steroids via heterogeneous SELEX using partially randomized DNA libraries with a pre-designed, three-way-junction binding domain. ${ }^{33}$ We believe that such a selection strategy could similarly be applied for the isolation of aptamers for other drugs of abuse as well as clinically relevant targets such as small-molecule biomarkers, toxins and therapeutics. Based on Heemstra's general approach to split three-way-junction structured aptamers ${ }^{92}$ and the simplicity of engineering a CBSA from a split aptamer, it should be straightforward to develop other CBSA-based assays from the isolated aptamers for which the target-binding domain has been identified. Since many optical and electrochemical sensing strategies have been developed that employ target-induced split aptamer assembly, ${ }^{94,160,203-205}$ it should be feasible to integrate our CBSA into these platforms. We also foresee the potential to improve the performance of our CBSA-based assay by employing signal amplification techniques ${ }^{160,204-207}$ that could further enhance its sensitivity. 
CHAPTER 4: Rapid and Sensitive Detection of Small-Molecule Targets Using

Cooperative Binding Split Aptamers and Enzyme-Assisted Target Recycling

\subsection{Introduction}

The biosensor field is on a continuous quest for ever-greater sensitivity. In conventional bioassays, where the signal is directly proportional to the target concentration, the sensitivity is determined by the intrinsic target affinity of the bioreceptor being used for detection. ${ }^{156}$ In this scenario, it would be difficult to generate a measurable signal at target concentrations more than 100 -fold lower than the $K_{D}$ of the bioreceptor. Accordingly, many amplification approaches have been developed in which one binding event can generate multiple signals, ${ }^{157,158}$ allowing detection of targets at very low concentrations. Enzyme-assisted target recycling (EATR) has proven to be an especially effective way to amplify signals generated from target-binding events. ${ }^{159}$ This approach relies on selective, nuclease-mediated degradation of the probe strand of a target-probe duplex that only forms in the presence of target; this liberates the target, which is 'recycled' for use in additional digestion reactions. For example, EATR has been used with molecular beacon probes for DNA detection. ${ }^{158}$ The molecular beacon probe contains a loop sequence complementary to the target DNA, with a fluorophorequencher pair attached to the stem of the beacon as a signal-reporting element. When the molecular beacon probe binds the target DNA, it switches from a stem-loop structure into a probe-target duplex that contains a structural ${ }^{164}$ or sequence ${ }^{158}$ recognition site for a nuclease. This enzyme then catalyzes the digestion or cleavage of the beacon probe, liberating the fluorophore and releasing the target, which can then hybridize with another molecular beacon probe. Thus, a substantial fluorescent signal can be generated by a 
single copy of the target though EATR, greatly decreasing the limit of detection. ${ }^{158,164}$ Given that DNA can be easily modified with various signaling reporters ${ }^{154,158,161,162}$ and that different nucleases can be utilized to process the target-probe duplex, ${ }^{158,161,163,164}$ many EATR-based assays have been developed for DNA detection with ultra-high sensitivity. ${ }^{159}$

Aptamers are nucleic acid-based bio-recognition elements that are isolated in vitro through SELEX processes. ${ }^{1}$ Aptamers can bind to a wide variety of targets, including proteins, metal ions, small molecules, and even whole cells, ${ }^{3}$ and aptamer-based biosensors have been developed for environmental monitoring, drug detection, and medical diagnostics. ${ }^{9-11}$ Aptamer-based sensors have many advantages compared to other state-of-the-art methods for the detection of small-molecule targets. Instrumental methods, such as gas or liquid chromatography/mass spectrometry, are sensitive and specific. However, these methods require laborious sample preparation and instruments that are cumbersome and sophisticated, limiting their use for on-site and high-throughput detection. ${ }^{12}$ Antibody-based immunoassays, such as ELISA, are highly sensitive and offer high target specificity. However, the in vivo processes for antibody generation are tedious, costly, and challenging for non-immunogenic small molecules. ${ }^{16}$ On the other hand, aptamers can be isolated rapidly with controllable affinity and specificity, and are produced without batch-to-batch variation. ${ }^{16}$ Many strategies have been employed into aptamer-based assays to achieve target detection in an instrument-free manner. ${ }^{208}$ In particular, aptamers can be split into two or three fragments that remain separate in the absence of target but assemble upon target binding, ${ }^{21,90}$ and such split-aptamer based sensors have gained popularity as a potential strategy for effective signal reporting. ${ }^{91}$ In 
principle, the target-induced assembly of split aptamers should be compatible with EATR-mediated signal amplification. Ideally, the split fragments should be highly responsive to target-induced assembly and only the target-aptamer complex should be specifically recognized and digested by the enzyme. However, it is difficult to achieve sensitive target-induced aptamer assembly with conventional split aptamers with a single binding-domain. Small-molecule-binding split aptamers usually have equilibrium dissociation constants $\left(\mathrm{K}_{\mathrm{D}}\right)$ in the high micromolar range, such that no measurable targetinduced aptamer assembly can be observed even with a high concentration of targets. ${ }^{92,95}$ Although target affinity can be improved by engineering split aptamers with longer complementary stems, the majority of thermo-stable split aptamers undergo some degree of pre-assembly in the absence of target, ${ }^{95}$ producing high background signal. Second, in contrast to DNA-based EATR assays, in which target-binding always converts a stemloop probe structure into a probe-target duplex, aptamer-target binding often gives rise to complex tertiary structures that are non-ideal substrates for nucleases. For example, three-way junctions and G-quadruplexes have been shown to inhibit exonuclease $\mathrm{I},{ }^{209}$ flap endonuclease $\mathrm{I}^{210}$ and polymerases. ${ }^{211}$ Additionally, target binding within the aptamer can interfere with enzyme binding and activity; for example, some smallmolecule DNA-intercalating agents and DNA adducts can inhibit nucleases, especially exonucleases. ${ }^{210,212,213}$

To overcome these limitations, we have developed a strategy for generating CBSAs for use in EATR-amplified biosensors. CBSAs are engineered from parent split aptamers with a single target-binding domain, and consist of a short fragment and a long fragment that contain two target-binding domains. The first binding event partially stabilizes the 
CBSA structure such that the second binding event can occur. This cooperative assembly can reduce the concentration change required to assemble the CBSA several-fold, resulting in far greater specificity and sensitivity relative to the parent split aptamer. ${ }^{95,186}$ For example, our group has previously demonstrated the successful engineering of a highly target-responsive CBSA for cocaine, which enables specific, ultra-sensitive, onestep fluorescence detection of cocaine within 15 minutes in $10 \%$ saliva. ${ }^{95}$ Here, we have developed a CBSA-based EATR-amplified fluorescence assay for the detection of dehydroisoandrosterone-3-sulfate (DIS) in urine samples. We modified our short fragment to incorporate a $\mathrm{C} 3$ spacer abasic site ${ }^{214}$ when the CBSA assembles, the resulting duplex at the abasic site is recognized and cleaved by exonuclease III (Exo III). This results in recycling of the long fragment and DIS molecules for further rounds of digestion, while releasing a fluorescent signal for each digestion event. We demonstrated that this assay exhibits 100 -fold enhanced sensitivity relative to the CBSA alone without EATR signal amplification. Importantly, this assay also proved highly sensitive when used in urine samples, allowing us to detect DIS in 50\% urine within 30 minutes at concentrations as low as $1 \mu \mathrm{M}$. To demonstrate the generality of our approach for the detection of other small-molecule targets, we also developed an assay that utilizes EATRmediated aggregation of CBSA fragment-modified gold nanoparticles (AuNPs) for colorimetric detection of cocaine. This assay was able to successfully achieve naked-eye detection of low micromolar concentrations of cocaine within 20 minutes. We believe that our approach should offer a general strategy for the development of other CBSAbased, EATR-amplified assays for the rapid, sensitive, and specific detection of various small-molecule targets in complex samples in both clinical and field settings. 


\subsection{Experimental section}

\subsubsection{Materials}

Table 4-1. The sequences of the oligonucleotide strands employed in Chapter 4.

\begin{tabular}{ll}
\hline Sequence ID & Sequence $\left(5^{\prime}-3^{\prime}\right)$ \\
\hline COC-LF & CTCCTTCAACGAAGTGGGTCTCCTTCAACGAAGTGGGTCT \\
COC-SF & CAAAAA \\
COC-SF-5'A & AAAAAGAAGG/iSpC3/GACAAGGAG \\
COC-SF-3'A & GAGACAAGG/iSpC3/GACAAGGAGAAAAA \\
COC-SF-T & AAAAAGAGACAAGGTGACAAGGAG \\
SH-COC-SF-1X & HS- $\left(\mathrm{CH}_{2}\right)_{6}$-TTTTTTGAGACAAGG/iSpC3/GACAAGGAG \\
SH-COC-SF-2X & HS- $\left(\mathrm{CH}_{2}\right)_{6}$-/TTTTTGAGACAAGG/iSpC3/GACAAGGAGTTTTT \\
& TGAGACAAGG/iSpC3/GACAAGGAG \\
DIS-LF & GTCCGCATACGAAGTTGTCTTCCGCATACGAAGTTGTCCAAAAA \\
DIS-SF & GGACGTGGA/iSpC3/GACGTGGACAAAAA \\
FQ-DIS-SF & /5IAbRQ/GGACGTGGA/iSpC3/GACG TGGAC/iCy5/AAAAA \\
DIS-LF-536 & GTCCGCATACGAAGTTGTCTTCCGCAAAAA \\
FQ-DIS-SF-536 & /5IAbRQ/GTGGA/iSpC3/GACGTGGAC/iCy5/AAAAA \\
DIS-LF-36 & GTCCGCATACGAAGTTGCAAAAA \\
FQ-DIS-SF-36 & /5IAbRQ/GACGTGGAC/iCy5/AAAAA \\
\hline \hline
\end{tabular}

a. /iSpC3/ represents internal C3 spacer modification

b. /5IAbRQ/ represents Iowa Black RQ quencher modification

c. /iCy5/ represents Cy5 fluorophore modification

All DNA strands used in this work were synthesized by Integrated DNA Technologies and purified with HPLC. DNA was dissolved in PCR grade water and DNA concentrations were measured with a NanoDrop 2000 (Thermo Scientific). The sequences of the oligonucleotide strands are listed in Table 4-1. Tris(hydroxymethyl)aminomethane (Tris), hydrochloric acid, sodium chloride, magnesium chloride, bovine serine albumin (BSA), 1,4-dithiothreitol (DTT), gold (III) chloride trihydrate, cocaine hydrochloride, and DIS were purchased from Sigma-Aldrich. Exo III was purchased from New England Biolabs. OliGreen and SYBR gold were 
purchased from Thermo Fisher Scientific. Urine samples used in this work were collected from healthy and consenting adult donors.

\subsubsection{Isothermal titration calorimetry (ITC)}

ITC experiment was performed with a MicroCal ITC200 (Malvern). $0.2 \mathrm{mM}$ DIS was titrated into $10 \mu \mathrm{M}$ DISS.1-AT, with both diluted in DIS binding buffer (10 mM Tris$\mathrm{HCl}, 0.5 \mathrm{mM} \mathrm{MgCl}_{2}, \mathrm{pH}$ 7.4). The experiment consisted of 19 successive $2 \mu \mathrm{L}$ injections after a $0.4 \mu \mathrm{L}$ purge injection with spacing of 180 seconds at $23{ }^{\circ} \mathrm{C}$. The injection heat was analyzed with the MicroCal analysis kit integrated into Origin 7 software, and the titration curve was fitted with a single-site binding model.

\subsubsection{Determining binding affinity of DIS-CBSA-4536, DIS-SA-536 and DIS-SA-36}

The binding affinities of the split aptamers were determined fluorescently as reported previously ${ }^{95}$ with some modifications. $1 \mu \mathrm{L}$ of $100 \mu \mathrm{M}$ DIS long fragment (DIS-LF, DISLF-536 or DIS-LF-36), $1 \mu \mathrm{L}$ of $100 \mu \mathrm{M}$ respective fluorophore/quencher-modified DIS short fragment (FQ-DIS-SF, FQ-DIS-SF-536 or FQ-DIS-SF-36), and $93 \mu \mathrm{L}$ of DIS binding buffer were mixed with $5 \mu \mathrm{L}$ of solution containing various concentrations of DIS. $80 \mu \mathrm{L}$ of each sample was then transferred into wells of a 96-well plate. After a 30minute incubation at room temperature, the fluorescence was measured using a TECAN M1000 Pro $(\lambda e x / e m=648 / 668 \mathrm{~nm})$. Each sample was analyzed in triplicate, and the means and standard deviations were plotted. The data were fitted with the Hill equation using OriginLab 9 to calculate Hill coefficient $\left(\mathrm{n}_{\mathrm{H}}\right)$ and $\mathrm{K}_{1 / 2}$ (DIS concentration producing half occupancy) of each split aptamer. 


\subsubsection{Exo III digestion and gel electrophoresis analysis}

All digestion experiments were performed as followed unless specified otherwise. 1 $\mu \mathrm{L}$ each of $50 \mu \mathrm{M}$ short and long CBSA fragments were added into $43 \mu \mathrm{L}$ of reaction buffer to yield a final DIS and cocaine concentrations of $250 \mu \mathrm{M}$ and $500 \mu \mathrm{M}$, respectively. The buffer composition varied for each aptamer/target composition, with final concentrations for each reaction as follows: DIS (10 mM Tris, $0.5 \mathrm{mM} \mathrm{MgCl}, 0.1$ $\mathrm{mg} / \mathrm{mL}$ BSA, $\mathrm{pH} 7.4)$ and cocaine (10 mM Tris, $0.1 \mathrm{mM} \mathrm{MgCl}_{2}, 0.1 \mathrm{mg} / \mathrm{mL}$ BSA, $\mathrm{pH}$ 7.4). After incubation at $23{ }^{\circ} \mathrm{C}$ for 30 minutes, $5 \mu \mathrm{L}$ of $0.01 \mathrm{U} / \mu \mathrm{L}$ Exo III was added.

After $5,10,15,30$ and 60 minutes of digestion, $5 \mu \mathrm{L}$ of each sample was collected and immediately mixed with $10 \mu \mathrm{L}$ of loading buffer $(71.25 \%$ formamide, $10 \%$ glycerol, $0.125 \% \mathrm{SDS}, 25 \mathrm{mM}$ EDTA, and $0.15 \%(\mathrm{w} / \mathrm{v})$ xylene cyanol) to inactivate Exo III. Control samples were prepared similarly, but with $5 \mu \mathrm{L}$ reaction buffer instead of Exo III. The digestion products were characterized via denaturing polyacrylamide gel electrophoresis (PAGE), with $3 \mu \mathrm{L}$ of each sample loaded into each well. Separation was carried out at $20 \mathrm{~V} / \mathrm{cm}$ for 3 hours in $0.5 \times$ TBE running buffer. The gel was stained with $1 \times$ SYBR Gold for 25 minutes and imaged using a ChemiDoc MP imaging system (BioRad). The percent of digestion was calculated based on band intensity relative to the control sample.

\subsubsection{CBSA-based, EATR-amplified fluorescence assay for detection of DIS}

$2 \mu \mathrm{L}$ of $100 \mu \mathrm{M}$ DIS-LF or DIS-LF-536, $1 \mu \mathrm{L}$ of $100 \mu \mathrm{M}$ FQ-DIS-SF or FQ-DIS-SF536, and $92 \mu \mathrm{L}$ of DIS binding buffer (10 mM Tris-HCl, $0.5 \mathrm{mM} \mathrm{MgCl} 2, \mathrm{pH} 7.4$ ) or $50 \%$ urine diluted with the binding buffer were mixed with $5 \mu \mathrm{L}$ of solution containing various concentrations of DIS in the wells of a 96-well plate. The fluorescence of each sample 
was first measured $\left(\lambda_{\mathrm{ex} / \mathrm{em}}=648 / 668 \mathrm{~nm}\right)$ after a 30 -minute incubation at room temperature. Then, $5 \mu \mathrm{L}$ of $0.2 \mathrm{U} / \mu \mathrm{L}$ Exo III was added into each well to initiate EATR. After a 30-minute digestion at room temperature, the fluorescence of each sample was measured again. The signal gain was calculated by $\left(\mathrm{F}-\mathrm{F}_{0}\right) / \mathrm{F}_{0} \times 100 \%$, where $\mathrm{F}_{0}$ is the fluorescence of the split aptamer mixtures without DIS and F is the fluorescence of split aptamer mixtures with different concentrations of DIS. Error bars were calculated from standard deviation of signal gains from three individual measurements at each DIS concentration.

\subsubsection{Synthesis of gold nanoparticles (AuNPs) modified with cocaine-binding}

\section{CBSA short fragment}

The modification of the thiolated short fragment onto 13-nm AuNPs was performed using a previously published protocol, ${ }^{143}$ at a 300:1 molar ratio of thiolated short fragment:AuNP. The modified AuNPs were then treated with DTT to regulate surface coverage. Specifically, $50 \mu \mathrm{L}$ of $5 \mathrm{nM}$ modified AuNPs were mixed with $20 \mu \mathrm{L}$ of solution containing different concentrations of DTT in $10 \mathrm{mM}$ Tris- $\mathrm{HCl}(\mathrm{pH} \mathrm{7.4)}$. After a 30 -minute incubation, the solution was centrifuged at $28,000 \times \operatorname{rcf}$ for 10 minutes. The supernatant was discarded to remove the excess DTT and displaced DNA strands, and the precipitated AuNPs were resuspended in $10 \mathrm{mM}$ Tris- $\mathrm{HCl}(\mathrm{pH}$ 7.4). This step was repeated twice. Finally, the modified AuNPs were resuspended in $5 \mu \mathrm{L}$ of the same buffer to obtain a particle concentration of $50 \mathrm{nM}$. 


\subsubsection{Quantitation of the surface coverage of CBSA short fragments on AuNPs via}

\section{DTT displacement}

We measured surface coverage of the modified AuNPs using a standard DTT displacement assay. ${ }^{141}$ The AuNPs were diluted to a final volume of $55 \mu \mathrm{L}$ in Tris buffer (10 mM Tris-HCl, pH 7.4). $50 \mu \mathrm{L}$ of AuNP solution was loaded into a well of a 384 -well plate and the absorbance was measured using a TECAN M1000 Pro. The concentration of AuNPs was calculated using Beer's law $\left(\varepsilon=2.7 \times 10^{8} \mathrm{~mol}^{-1} \cdot \mathrm{cm}^{-1}\right) .50 \mu \mathrm{L}$ of the modified AuNP solution was collected and mixed with $50 \mu \mathrm{L}$ of $1.0 \mathrm{M}$ DTT solution. After an overnight incubation at room temperature, the solution was centrifuged at $25,000 \times$ rcf for 10 minutes to remove the AuNP precipitate. $20 \mu \mathrm{L}$ of the supernatant was then mixed with $80 \mu \mathrm{L}$ of $0.625 \times$ OliGreen solution to obtain a $0.5 \times$ Oligreen concentration. $100 \mu \mathrm{L}$ of supernatant-Oligreen mixture was loaded into a well of a 384well microplate and fluorescence was measured using a TECAN M1000 Pro $\left(\lambda_{\text {ex } / \mathrm{em}}=\right.$ 500/525 nm). DTT-displaced oligonucleotides were quantified using an established calibration curve derived from CBSA short fragments that had been subjected to the same DTT treatment.

\subsubsection{CBSA-based, colorimetric EATR assay for detection of cocaine}

$2.5 \mu \mathrm{L}$ of short-fragment-modified AuNPs $(50 \mathrm{nM}), 1 \mu \mathrm{L}$ of $2.5 \mu \mathrm{M}$ long fragment (COC-LF), $2 \mu \mathrm{L}$ of a solution with different cocaine concentrations or different cutting agents with final concentration of $50 \mu \mathrm{M}$, and $15.5 \mu \mathrm{L}$ reaction buffer (final concentrations: $10 \mathrm{mM}$ Tris- $\mathrm{HCl}, 100 \mathrm{mM} \mathrm{NaCl}, 0.75 \mathrm{mM} \mathrm{MgCl}_{2}, 0.1 \mathrm{mg} / \mathrm{mL}$ BSA, $\mathrm{pH}$ 7.4) were mixed in wells of a 384 -well plate. After 30 minutes, $4 \mu \mathrm{L}$ of $1.25 \mathrm{U} / \mu \mathrm{L}$ Exo III 
was added into each well. Absorbance spectra from 400-800 nm were recorded every 5 minutes, with pictures taken by Nikon D800 after 20 minutes of Exo III digestion.

\subsection{Results and discussion}

\subsubsection{Engineering of DIS-binding CBSA}

Stojanovic and colleagues recently isolated aptamers for various steroids, including DIS, using a library-immobilized SELEX approach. ${ }^{33}$ DIS is one of the most abundant steroid hormones in urine. The concentration of DIS in healthy adults typically ranges from $1-20 \mu \mathrm{M},{ }^{170,171}$ but can climb to the sub-millimolar range in patients with adrenal tumors. ${ }^{172}$ The isolated DIS-binding aptamer (DISS.1-AT) demonstrated a $\mathrm{K}_{\mathrm{D}}$ of $0.44 \mu \mathrm{M}$ (Figure 4-1), and we used DISS.1-AT as a starting point for our CBSA engineering strategy that we have described previously. ${ }^{95}$ First, we generated parent split aptamers by removing the poly $(\mathrm{T})_{3}$ loop region from DISS.1-AT and reducing the number of base pairs in stem 1 (Figure 4-2A). We then fabricated a DIS-binding CBSA by fusing two sets of these parent split aptamers as shown in Figure 4-2B. The engineered CBSA consists of a short fragment and a long fragment. To facilitate EATR, we replaced the adenosine at position 9 from the 5 ' end of the short fragment with a $\mathrm{C} 3$ spacer abasic site (labeled as X in Figure 4-2C). In the presence of DIS, these two fragments assemble to form DIS-CBSA-4536 complex (Figure 4-2D), where the C3 spacer abasic site is opposite a thymine within the long fragment. Exo III can cleave the short fragment at this duplexed abasic site using its apurinic endonuclease activity, ${ }^{214}$ releasing the intact long fragment and DIS molecules. 


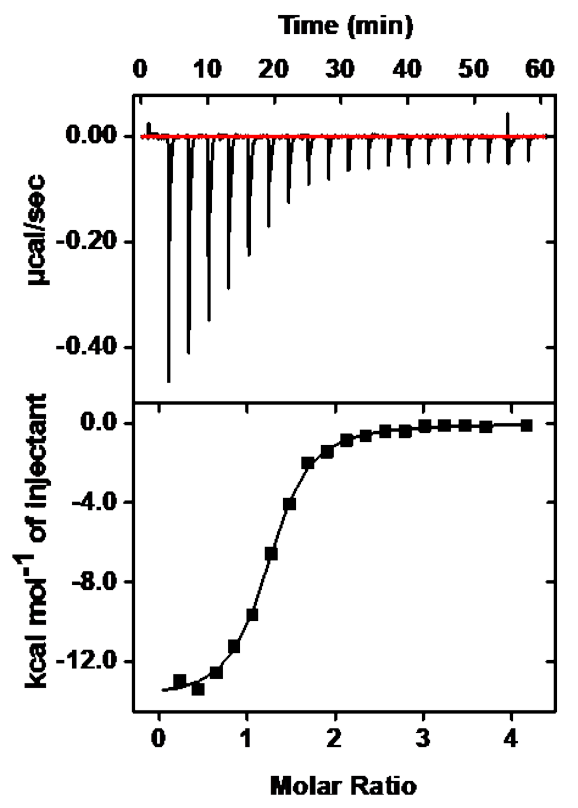

Figure 4-1. Characterization of DIS binding affinity of DISS.1-AT using ITC. Top panel presents raw data showing the heat generated from each titration of DIS for DISS.1-AT. Bottom panel shows the integrated heat of each titration after correcting for dilution heat of the titrant.

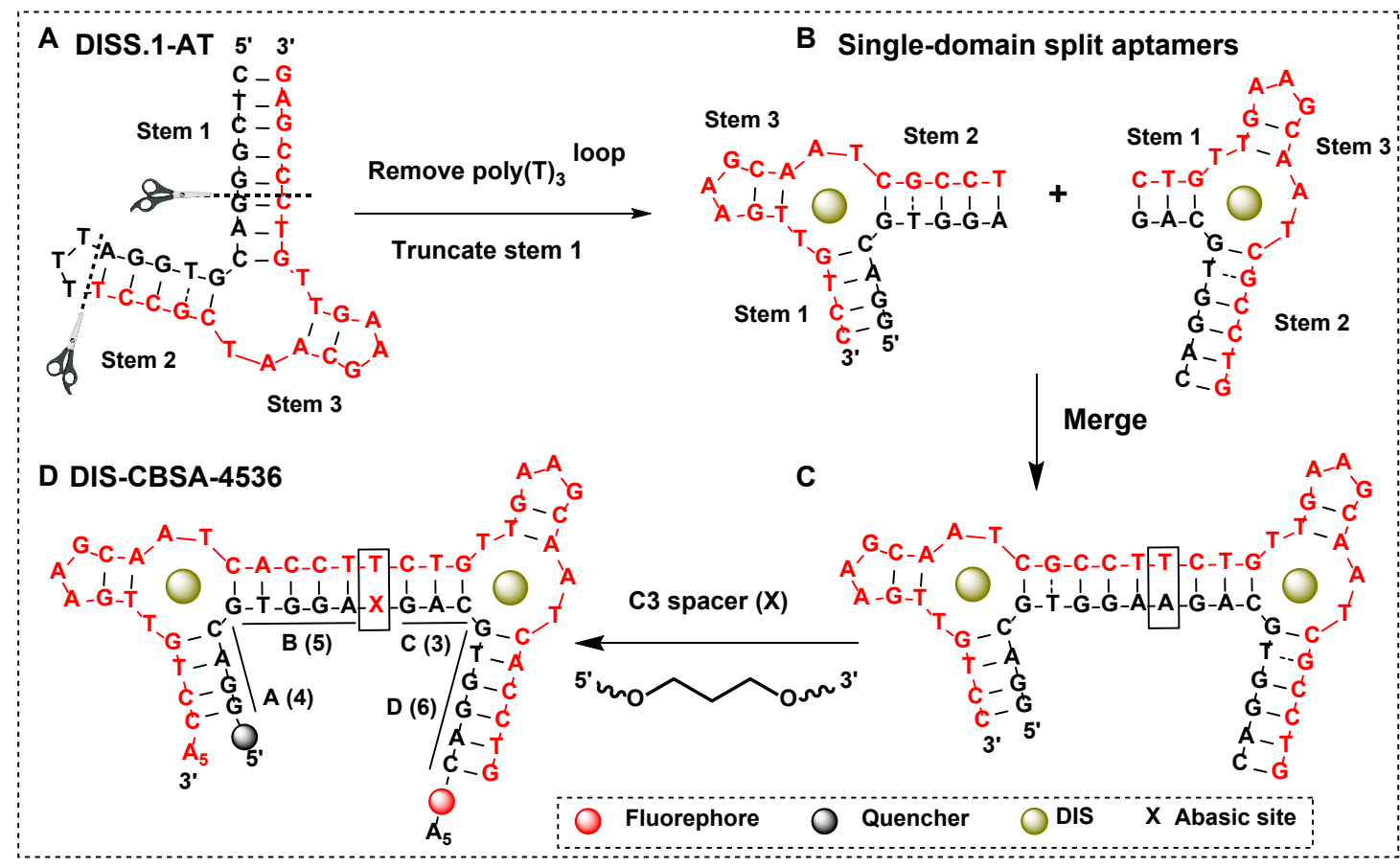

Figure 4-2. Design of DIS-binding CBSA. (A) The single-stranded DIS binding aptamer (DISS.1-AT) was truncated at Stem 1 and Stem 2 to generate the parent split aptamers. (B) Two sets of parent split aptamers were $(\mathbf{C})$ merged and modified to incorporate (D) a C3 spacer abasic site (denoted by $\mathrm{X}$ ) that resides opposite a thymine nucleotide when the CBSA duplex is assembled. A fluorophore (Cy5) and a quencher (Iowa Black RQ) were respectively modified at the 3' and 5' terminus of the short CBSA fragment. A poly(A) 5 overhang was added on the 3' termini of each CBSA fragment to prevent non-specific Exo III digestion. 


\subsubsection{Design of a CBSA-based EATR-amplified fluorescence assay}

To confirm binding cooperativity of DIS-CBSA-4536, we modified the short fragment with a Cy5 fluorophore and an Iowa Black RQ quencher at the 3' and 5' terminus, respectively (FQ-DIS-SF) (Figure 4-2D). In the absence of the target, both FQ-DIS-SF and the corresponding long fragment (DIS-LF) remain separate, and the fluorophore is quenched due to its close proximity with the quencher (Figure 4-3A). Upon the addition of DIS, the two fragments assemble to form a rigid CBSA-target complex, separating the fluorophore from the quencher and resulting in elevated fluorescence (Figure 4-3B). By titrating various DIS concentrations, we determined a Hill coefficient $\left(\mathrm{n}_{\mathrm{H}}\right)^{192}$ of 1.6 for DIS-CBSA-4536 (Figure 4-4A), indicating considerable positive cooperativity. This implies that a transition of CBSA assembly from $10 \%$ to $90 \%$ requires only a 16 -fold increase in target concentration, compared to its parent split aptamers which require an 81-fold increase. ${ }^{186}$ NUPACK analysis $^{81}$ of the DIS-binding CBSA sequences demonstrated that both fragments are predominantly unassembled (99\%) in the absence of target under our experimental conditions but DIS-SF exists primarily as a duplexed structure. Therefore, binding of the first target to DIS-CBSA-4536 should open up the DIS-SF duplex, facilitating binding of a second DIS molecule to the CBSA. This results in a high level of cooperativity. A $\mathrm{K}_{1 / 2}{ }^{192}$ of $491 \mu \mathrm{M}$ was obtained, where $\mathrm{K}_{1 / 2}$ represents the DIS concentration at which half of the binding domains are occupied (Figure 4-4A). To demonstrate the highly sensitive target-induced assembly of the CBSA, we designed two sets of DIS-binding split aptamers with a single binding domain. Specifically, we extended Stem 1 of the DIS-binding parent split aptamer (termed DIS-SA-36) (Figure 4-2B, right and Figure 4-4B) by one abasic site and five base pairs to form a split aptamer 
with a longer stem (termed DIS-SA-536) (Figure 4-4C). The long fragments were unmodified while the short fragments were modified with a Cy5 fluorophore and an Iowa Black RQ quencher at the 3' and 5' terminus, respectively. We performed binding experiments with these two split aptamers along with the CBSA. DIS-CBSA-4536 demonstrated no background assembly in the absence of the target, and progressive target-induced assembly with increasing concentrations of DIS. DIS-SA-536 also showed target-induced assembly with a moderately higher target affinity, but had high background assembly in the absence of DIS (Figure 4-4A). As expected, no cooperativity was observed $\left(\mathrm{n}_{\mathrm{H}}=1.05\right)$ with this single-binding pocket split aptamer. The parent split aptamer, DIS-SA-36, was unable to assemble regardless of the presence or absence of target due to its thermal instability.

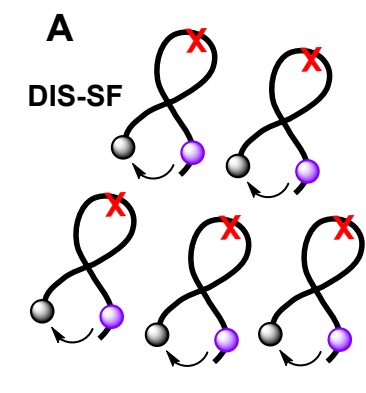

Fluorescence off

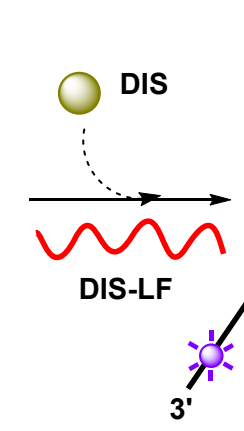

B
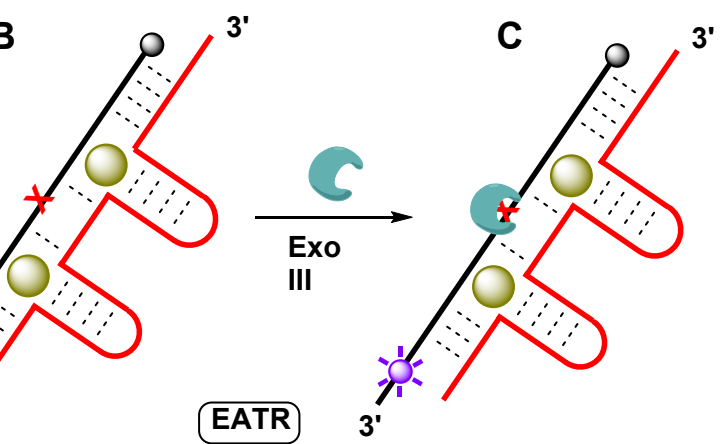

E

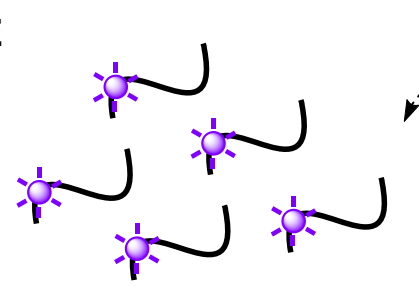

Fluorescence on
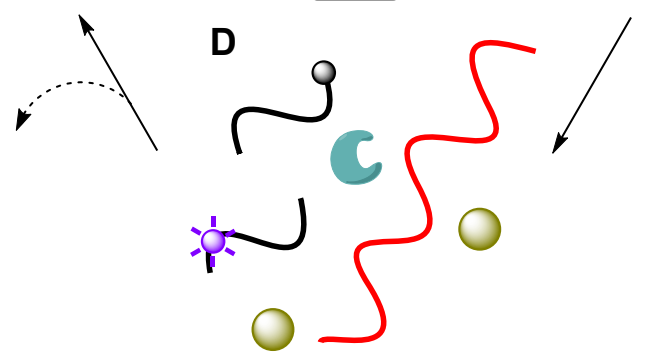

Figure 4-3. Working principle of our CBSA-based, EATR-amplified fluorescence assay. (A) FQDIS-SF contains a fluorophore-quencher pair, and remains dark in the absence of target. (B) In the presence of target, the CBSA assembles, $(\mathbf{C})$ creating a duplex that can be recognized and cleaved by Exo III at the abasic site (denoted by X). (D) The complex then disassembles, releasing the intact long fragment and target for additional rounds of complex formation and digestion. The fluorophore is also released and produces a signal, which (E) becomes amplified over the course of many rounds of EATR. 


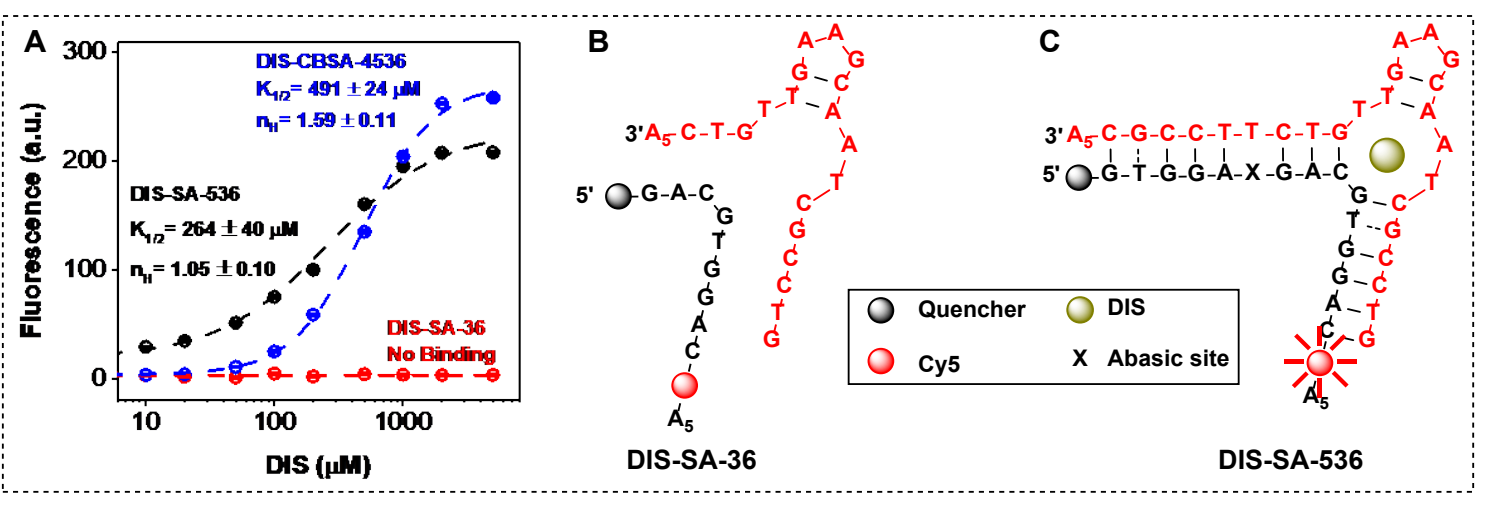

Figure 4-4. Characterization of target-induced assembly of DIS-CBSA-4536, DIS-SA-36 and DIS-SA-536 using a fluorophore-quencher assay. (A) Binding curve of these three split aptamers with DIS ranging from 0 to $5000 \mu \mathrm{M}$. Binding affinity $\left(\mathrm{K}_{1 / 2}\right)$ and cooperativity $\left(\mathrm{n}_{\mathrm{H}}\right)$ was characterized by fitting with the Hill Equation. The error bars represent the standard deviation of three measurements. Structures of (B) DIS-SA-36 and (C) DIS-SA-536 are given.

The affinity of DIS-CBSA-4536 was too low for direct detection of DIS at physiological levels. ${ }^{170,171}$ Nevertheless, the highly target-responsive feature of the CBSA enables us to demonstrate its compatibility with EATR-mediated signal amplification. We selected Exo III for this reaction because of its potent apurinic endonuclease activity, along with the fact that this enzyme is inexpensive and highly active at room temperature in a sequence-insensitive manner. ${ }^{215,216}$ When the CBSA-DIS complex assembles in the presence of DIS, Exo III is able to recognize the duplexed structure and specifically cleaves it at the abasic site (Figure 4-3C). This cleavage results in disassembly of the CBSA-target complex, releasing the long fragment, DIS molecules, and the fluorophore, which now fluoresces due to its separation from the quencher. The released long fragment and target are then free to assemble with another FQ-DIS-SF (Figure 4-3D), starting the cycle anew. The end result is that a very small amount of target can be recycled for multiple rounds of assembly and cleavage, generating a substantially amplified fluorescence signal (Figure 4-3E). 


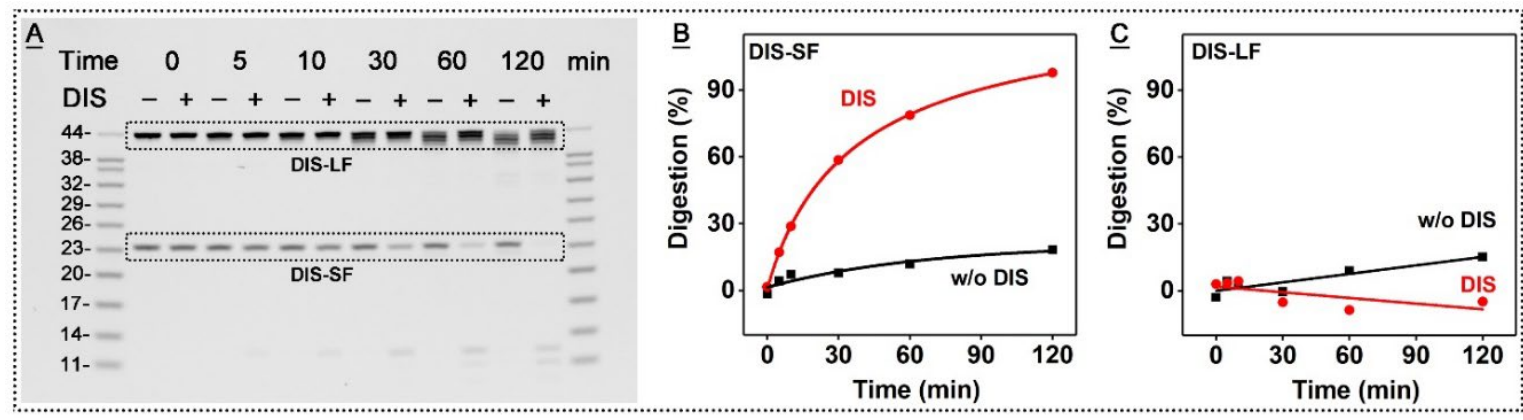

Figure 4-5. Time-course of Exo III-mediated cleavage of DIS-CBSA-4536. (A) PAGE analysis of digestion products from DIS-CBSA-4536. Reactions consisted of $1 \mu \mathrm{M}$ DIS-SF, $1 \mu \mathrm{M}$ DIS-LF and $0.004 \mathrm{U} / \mu \mathrm{L}$ Exo III with or without $500 \mu \mathrm{M}$ DIS after 5, 10, 15, 30, 60 and 120 minutes of digestion. Cleavage of short $(\mathbf{B})$ and long $(\mathbf{C})$ fragments was quantified by (Int 0 -Int)/Int $\mathrm{I}_{0} \times 100 \%$, where Int is the band intensity after the addition of Exo III and Int ${ }_{0}$ is the band intensity before the addition of Exo III.

\subsubsection{Exo III efficiently mediates EATR of DIS-CBSA-4536 in solution}

We then set out to demonstrate efficient Exo III digestion of abasic-site-modified DIS-SF. In order to prevent nonspecific digestion ${ }^{215}$ of the CBSA, we added a poly(A) 5 overhang at the 3' terminus of both fragments. We then performed a time-course of digestion with Exo III using DIS-CBSA-4536 in the presence or absence of DIS. The digestion products were collected after 5,10,15, 30, 60 and 120 minutes and analyzed by polyacrylamide gel electrophoresis (PAGE) (Figure 4-5A). The concentration of retained short fragments was calculated relative to a control sample that was untreated with Exo III. We observed that Exo III cleavage of DIS-SF was rapid and specific, with 59\% cleavage in the presence of $500 \mu \mathrm{M}$ DIS after 30 minutes. In contrast, only $9 \%$ of DIS-SF was cleaved in the absence of the target (Figure 4-5B). After 120 minutes, DIS-SF was almost completely cleaved (95\%) in the DIS sample, compared to only $15 \%$ cleavage in the absence of the target (Figure 4-5B). These results confirmed that the apurinic endonuclease activity of Exo III could specifically cleave the CBSA even when it is bound to the DIS target. Notably, we observed moderate non-specific digestion of DIS- 
LF by Exo III, resulting in partial removal of its poly(A) $)_{5}$ protection (Figure 4-5A). ${ }^{215}$ The total concentration of DIS-LF and its products, calculated from the intensity of all bands larger than $39 \mathrm{nt}$, remained constant after a 120-minute digestion (Figure 4-5C). Since all digested products contained the intact recognition segments of the CBSA, we believe that EATR efficiency was not affected. We then demonstrated recycling of target and long fragment by performing an Exo III cleavage reaction with $1 \mu \mathrm{M}$ DIS-LF and 4 $\mu \mathrm{M}$ DIS-SF in the presence of DIS. PAGE analysis confirmed that Exo III achieved efficient and specific cleavage of the short fragment, with $99 \%$ of DIS-SF cleaved after 240 minutes (Figure 4-6A). In contrast, only $11 \%$ of DIS-SF was cleaved in the absence of DIS (SI, Figure 4-6B). We observed that less than $15 \%$ of DIS-LF was digested regardless of the presence or absence of target (Figure 4-6C). Since the short fragment was almost completely cleaved despite being present at a concentration greatly exceeding that of the long fragment, it is clear that Exo III-mediated recycling of the long fragment and target was taking place.

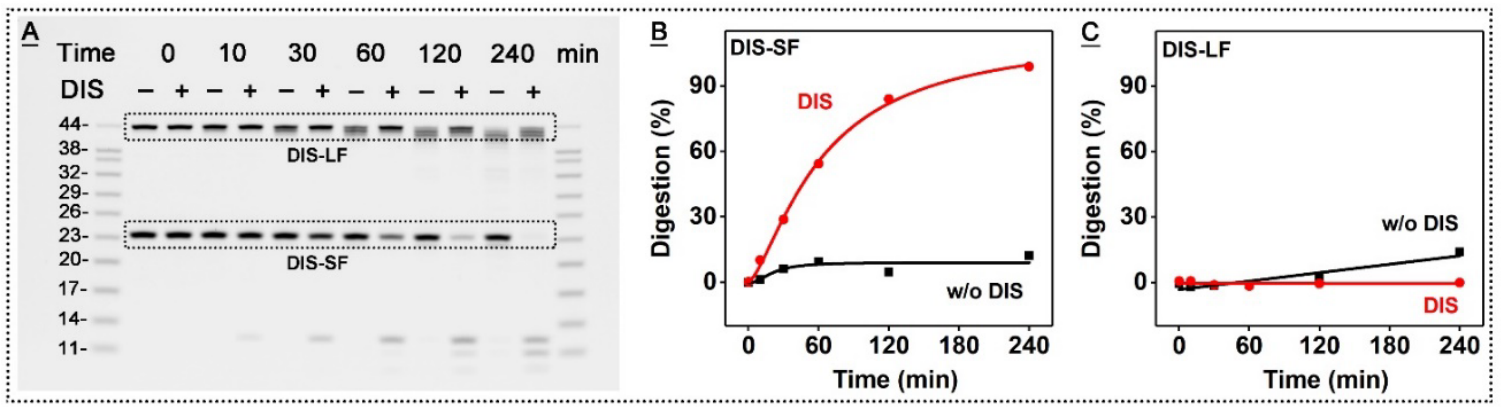

Figure 4-6. Digestion time-course of DIS-CBSA-4536 with excess DIS-SF. (A) PAGE analysis of digestion products from $4 \mu \mathrm{M}$ DIS-SF and $1 \mu \mathrm{M}$ DIS-LF in the presence of $0.1 \mathrm{U} / \mu \mathrm{L}$ Exo III with or without $500 \mu \mathrm{M}$ DIS after 10, 30, 60, 120 and 240 min of digestion. Digestion of DIS-SF (B) and DIS-LF (C) was quantified by (Int - -Int) $/ \operatorname{Int}_{0} \times 100 \%$, where Int is the band intensity after addition of Exo III and Int $_{0}$ is the band intensity before addition of Exo III. 

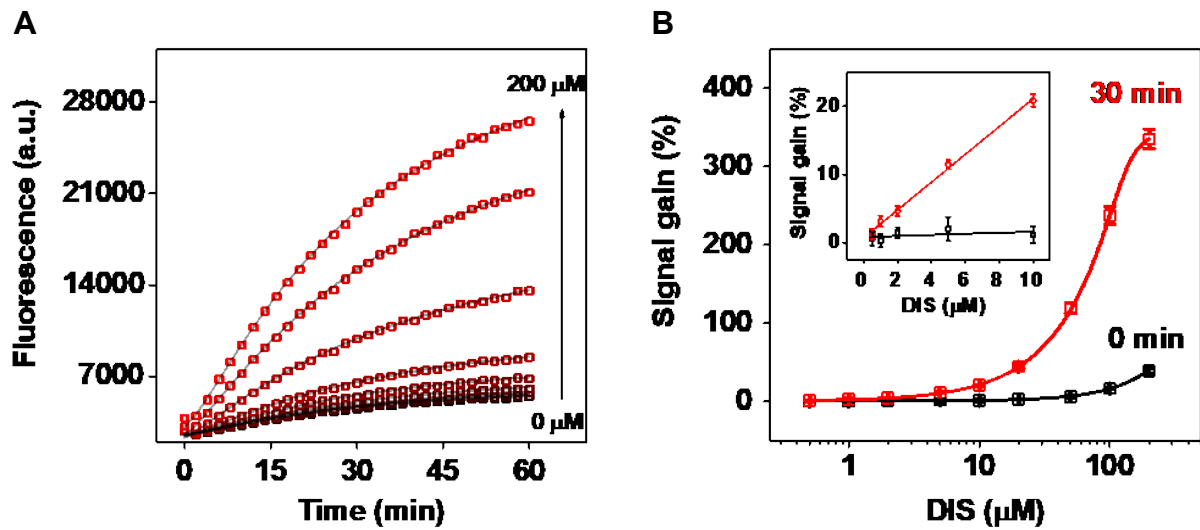

Figure 4-7. CBSA-based EATR-amplified fluorescence assay for sensitive detection of DIS. (A) Fluorescence time-course measurements after adding $0.01 \mathrm{U} / \mu \mathrm{L}$ of Exo III to our CBSA in the presence of different concentrations of DIS $(0,0.5,1,2,5,10,20,50,100$ and $200 \mu \mathrm{M})$. (B) Calibration curves before (black) and 30 minutes after (red) EATR signal amplification. The inset shows the calibration curve at low DIS concentrations. The signal gain was calculated from the fluorescence intensity of the samples. Error bars represent the standard deviation of three measurements. Limit of detection is determined using the lowest non-zero calibrator concentration. ${ }^{187}$

A

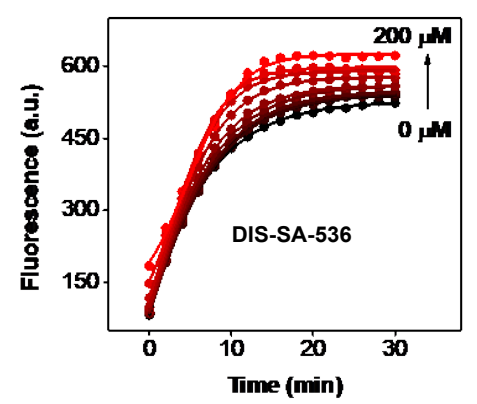

B

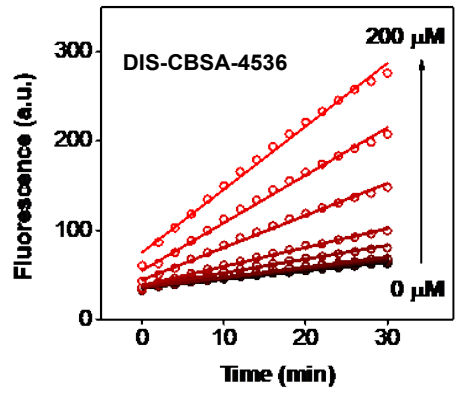

C

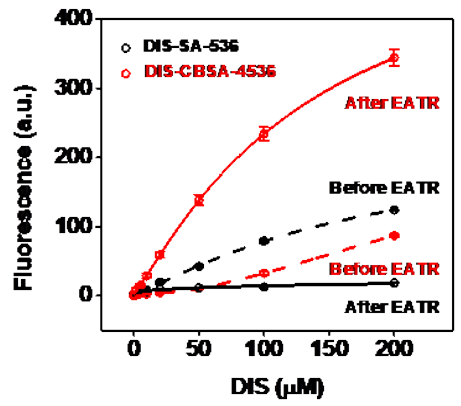

Figure 4-8. Demonstration of EATR-amplified signal in a fluorophore-quencher assay. Time course digestion of (A) DIS-SA-536 and (B) DIS-CBSA-4536 with 0, 0.5, 1, 2, 5, 10, 20, 50, 100, and $200 \mu \mathrm{M}$ DIS. (C) Calibration curves of the fluorophore-quencher assay obtained with both split aptamers before and after 30 minutes of EATR amplification.

\subsubsection{Sensitive DIS Detection in biofluid samples using CBSA-based EATR-}

\section{amplified fluorescence assay}

Having demonstrated the feasibility of our CBSA-based EATR-amplified approach, we performed the fluorescence assay described in Figure 4-3 to detect DIS. As expected, when we incubated $1 \mu \mathrm{M}$ FQ-DIS-SF with $2 \mu \mathrm{M}$ DIS-LF and different concentrations of DIS for 30 minutes, we did not observe a significant DIS-induced fluorescence change 
even with $100 \mu \mathrm{M}$ DIS (Figure 4-7, A and B, 0 min), as target binding of the CBSA was too weak to efficiently assemble the CBSA-target complex. However, after adding 0.01 $\mathrm{U} / \mu \mathrm{L}$ of Exo III, we saw a steady and concentration-dependent increase in fluorescence (Figure 4-7A). The initial rate at which fluorescence increased was proportional to the DIS concentration, and we found that concentrations as low as $1 \mu \mathrm{M}$ could be detected after 30 minutes of cleavage (Figure 4-7B, inset). It should be noted that the fluorescence of the samples without DIS also increased to some extent due to the nonspecific digestion of FQ-DIS-SF (Figure 4-7A, $0 \mu \mathrm{M}$ ). These results clearly demonstrated that the sensitivity of our CBSA binding assay was enhanced 100-fold by implementing EATR, producing a measurable limit of detection that is more than 500 -fold lower than the $\mathrm{K}_{1 / 2}$ of the CBSA. As a control experiment, we also performed our EATR-amplified fluorescence assay using DIS-SA-536. Although DIS-SA-536 underwent target-induced assembly and was efficiently cleaved by Exo III, we observed no target-induced signal amplification after a 30-minute digestion because of the high levels of DIS-independent background assembly (Figure 4-8, A and C). In contrast, DIS-CBSA-4536 exhibited clear target-induced signal amplification (Figure 4-8, B and C), with rapid assembly occurring at very low concentrations of target and minimal background assembly in the absence of target. Importantly, our assay performed equally well in urine samples. Different concentrations of DIS were spiked into $50 \%$ diluted urine and tested with our CBSA fluorescence assay. After a 30-minute enzyme digestion, we obtained a calibration curve with a linear range from 0 to $100 \mu \mathrm{M}$ (Figure 4-9A), which covers the urinary DIS concentration range in normal adults $(1-50 \mu \mathrm{M})$. In addition, we found that concentrations as low as $1 \mu \mathrm{M}$ DIS spiked in 50\% urine could be distinguished from the 
background signal (Figure 4-9B). Our results clearly demonstrated the robustness and sensitivity of our CBSA-based EATR fluorescence assay even in complex samples.
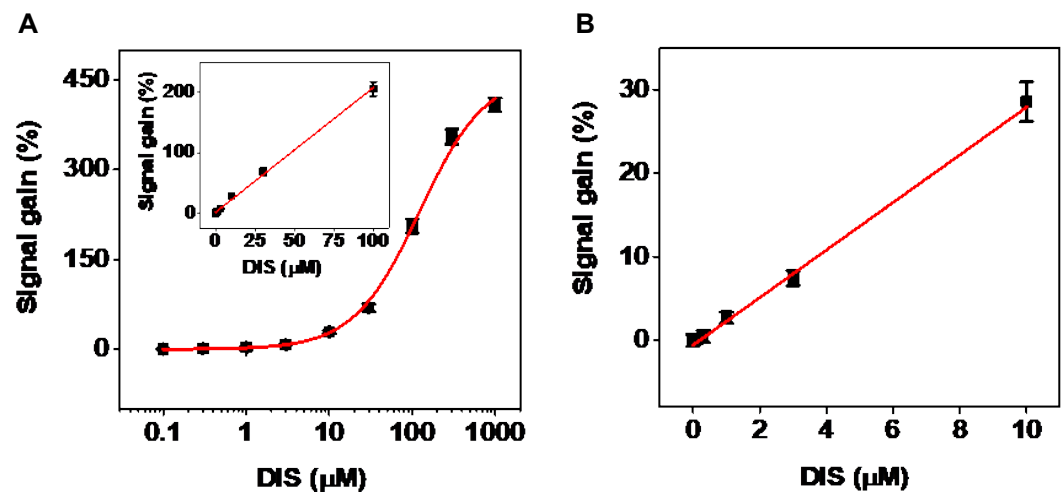

Figure 4-9. CBSA-based EATR-amplified fluorescence assay for sensitive detection of DIS in $50 \%$ urine. (A) Calibration curve after a 30-min enzyme digestion with a linear range between 0 and $100 \mu \mathrm{M}$ DIS (inset). (B) Calibration curve at lower DIS concentrations. The signal gain was calculated from the fluorescence intensity of the samples. Error bars represent the standard deviation of three measurements.

\subsubsection{Design of the CBSA-based colorimetric assay employing EATR-mediated}

\section{AuNP aggregation}

Due to the highly responsive target-induced assembly feature of CBSAs, we believe that a similar EATR-amplified strategy can be readily adopted into different sensing platforms using other small-molecule-binding CBSAs. For example, the EATR-amplified assay described above is instrument-dependent, making it unsuitable for instrument-free applications. However, the same strategy can be also used to achieve rapid colorimetric detection of small-molecule targets in an instrument-free fashion. As a demonstration, we used the cocaine-binding CBSA previously developed by our group, COC-CBSA-5335, which has an $n_{H}$ of 1.5 and a $K_{1 / 2}$ of $36 \mu M{ }^{95}$ to develop an assay with a visual readout that is potentially suitable for field use. Specifically, we immobilized the short fragment of this CBSA (COC-SF) onto AuNPs, and then added the free long fragment (COC-LF) in solution (Figure 4-10A). In the presence of cocaine, the fragments assemble to form 
CBSA-cocaine complexes on the particle surface (Figure 4-10B). Exo III specifically recognizes the duplexed $\mathrm{C} 3$ spacer abasic site between the two binding domains and cleaves the AuNP-conjugated short fragment (Figure 4-10C), releasing the intact long fragment and cocaine for another round of target assembly and Exo III cleavage (Figure 4-10D). Once all of the short fragments have been cleaved from the particle surface (Figure 4-10E), the AuNPs lose their stability under the employed buffer conditions and rapidly aggregate, producing a visible red-to-blue color change (Figure 4-10F). The CBSA fragments cannot assemble in the absence of cocaine, precluding Exo III digestion, and the intact short fragments prevent AuNP aggregation such that the solution remains red.

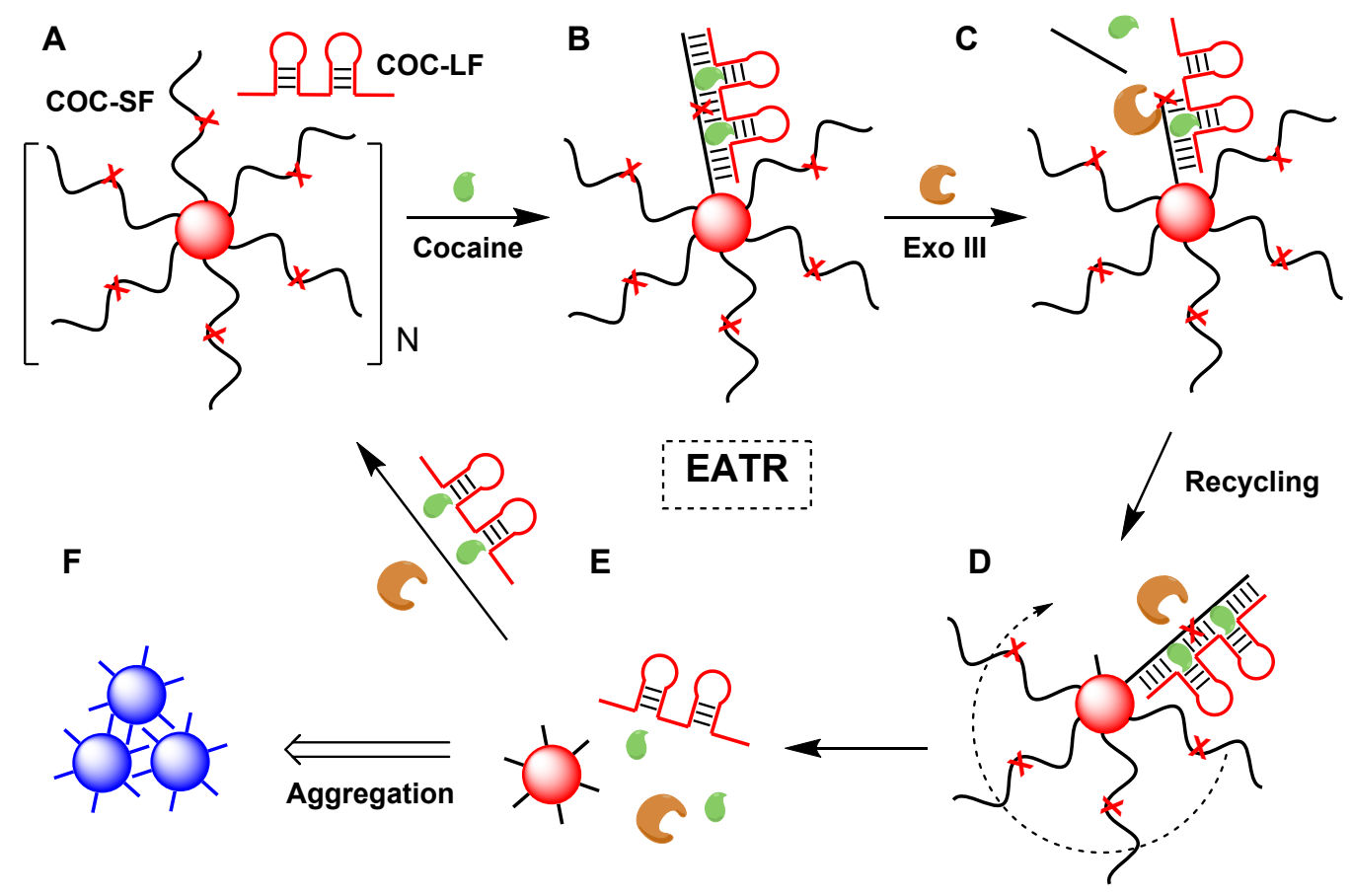

Figure 4-10. A colorimetric CBSA-based EATR-amplified assay for naked-eye detection of cocaine. (A) AuNPs are coupled to the short fragment (COC-SF), creating steric repulsion between AuNPs that produces a visible red color. Upon addition of long fragment (COC-LF) and cocaine, (B) the CBSA assembles, and the resulting duplexed abasic site can then $(\mathbf{C})$ be digested by Exo III. (D) This cleaves off COC-SF while recycling COC-LF and cocaine for another round of assembly and digestion, until (E) all short fragments have been sheared from the AuNP. (F) These sheared AuNPs can now aggregate, producing a visible red-to-blue color change. 


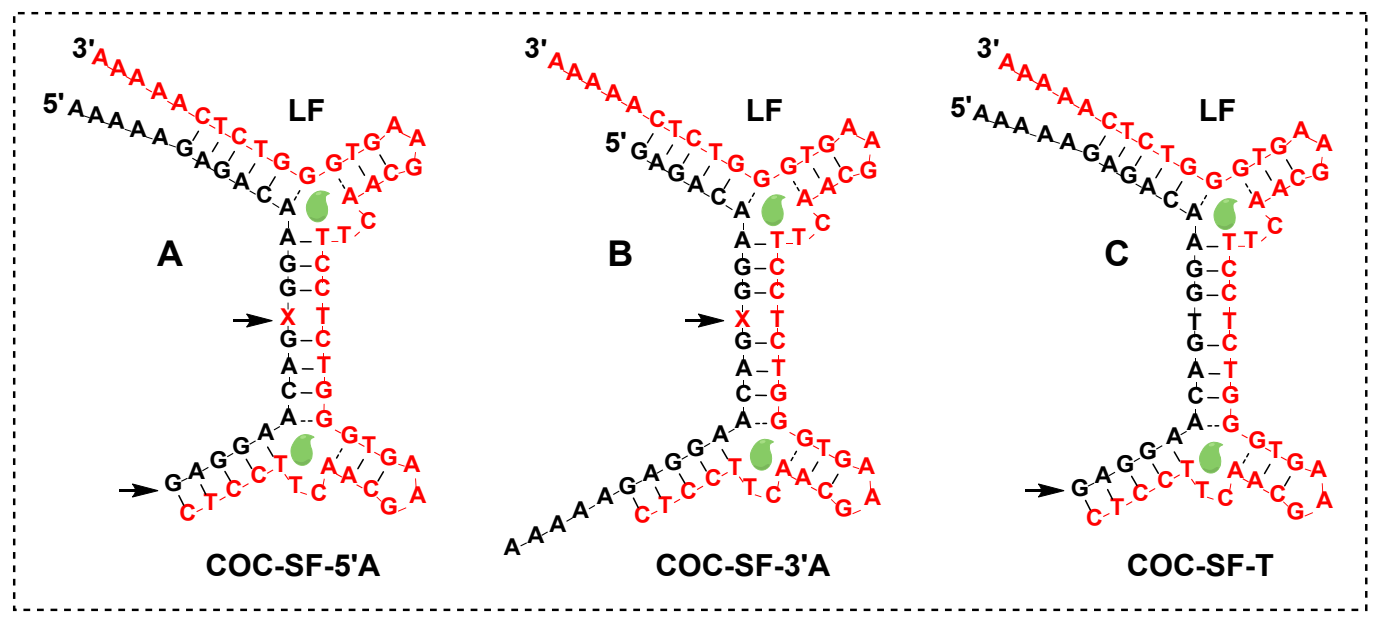

Figure 4-11. Cocaine-binding CBSA with a poly(A) $)_{5}$-protected long fragment and different derivatives of the short fragment. Arrows indicate suitable digestion sites for Exo III.

\subsubsection{EATR of CBSA mediated by AP endonuclease of Exo III instead of its 3'-5' exonuclease activity}

We first investigated the contribution of Exo III's 3'-5' exonuclease activity to the digestion of CBSA-cocaine complexes relative to its endonucleolytic activity by engineering three derivatives of COC-SF. The first derivative (COC-SF-5'A) included a poly $(\mathrm{A})_{5}$ extension at the 5 ' end that allows digestion of both the abasic site and the 3' terminus (Figure 4-11A). For the second derivative, the poly $(\mathrm{A})_{5}$ protection was added at the 3 ' instead of 5' end (COC-SF-3'A), allowing cleavage only at the abasic site (Figure 4-11B). For the third derivative (COC-SF-T), we replaced the abasic site with a thymine, allowing digestion only from the 3 ' terminus (Figure 4-11C). We also added a poly(A)5 extension at the 5' end of COC-SF-T to match the length of COC-SF-3'A. We performed Exo III digestion of a mixture of COC-LF with a 3' poly(A) $)_{5}$ modification combined with each of the three different short fragment derivatives in the presence or absence of 250 $\mu \mathrm{M}$ cocaine, and characterized the digestion products using PAGE. For the COC-SF-5'A, we observed that $61 \%$ and $5 \%$ of the short fragment was cleaved with and without 250 
$\mu \mathrm{M}$ cocaine, respectively, after a five-minute enzyme reaction (Figure 4-12). After 60 minutes, the amount of cleaved COC-SF-5'A increased to $97 \%$ and $21 \%$, respectively. We obtained a similar digestion profile for COC-SF-3'A, where Exo III cleavage occurs only at the abasic site. $61 \%$ and $8 \%$ of the COC-SF-3'A were respectively cleaved with and without addition of cocaine after five minutes of digestion, which increased to $95 \%$ and 17\%, respectively, after 60 minutes (Figure 4-13). These results demonstrated that the apurinic endonuclease activity of Exo III is efficient enough to mediate the digestion of CBSA-cocaine complexes. Our control experiment with COC-SF-T demonstrated that Exo III was able to remove only one nucleotide from the short fragment. Only $5 \%$ of the COC-SF-T was digested both with and without cocaine after a five-minute Exo III reaction, and only $15 \%$ of the COC-SF-T was digested in the presence of cocaine versus $20 \%$ without cocaine after 60 minutes (Figure 4-14). It is clear that the CBSA-cocaine complex inhibits the 3'-to-5' exonuclease activity of Exo III. Thus, we concluded that the abasic site is sufficient to enable robust EATR signal amplification, and poly(A)s protection at the $3^{\prime}$ end of the short fragment is unnecessary.
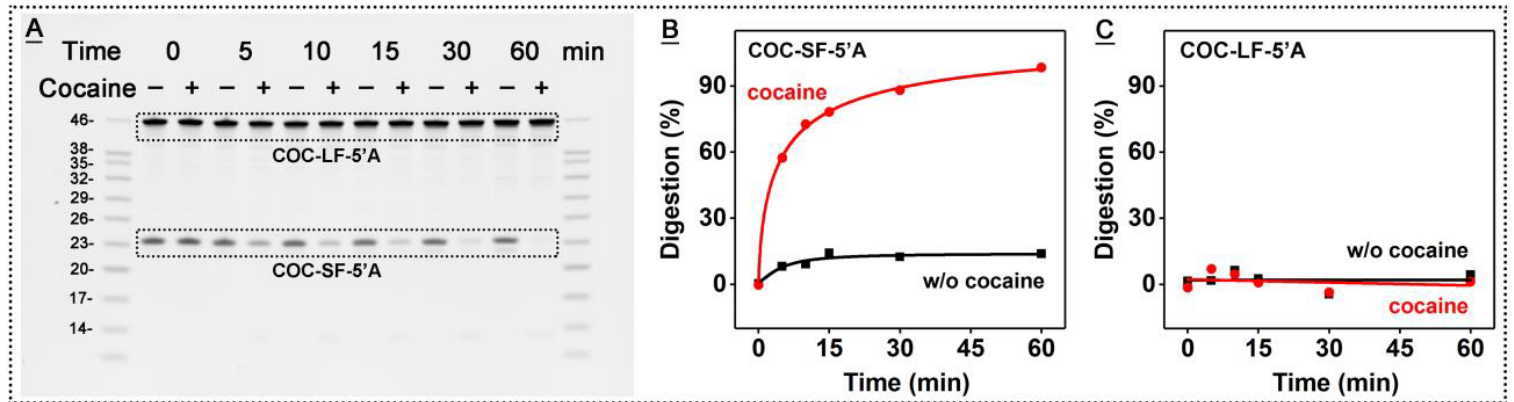

Figure 4-12. Time-course of CBSA digestion with COC-SF-5'A. (A) PAGE analysis of digestion products of CBSA consisting of $1 \mu \mathrm{M}$ COC-SF-5' A and $1 \mu \mathrm{M}$ poly(A) $)_{5}$ protected COC-LF after treatment with $0.01 \mathrm{U} / \mu \mathrm{L}$ Exo III in the presence or absence of $250 \mu \mathrm{M}$ cocaine for $5,10,15,30$ or $60 \mathrm{~min}$ of digestion. Digestion of (B) COC-SF-5'A and (C) COC-LF-5'A was quantified by $\left(\right.$ Int $_{0}$-Int $) /$ Int $_{0} \times 100 \%$, where Int is the band intensity after addition of Exo III and Int $_{0}$ is the band intensity before addition of Exo III. 


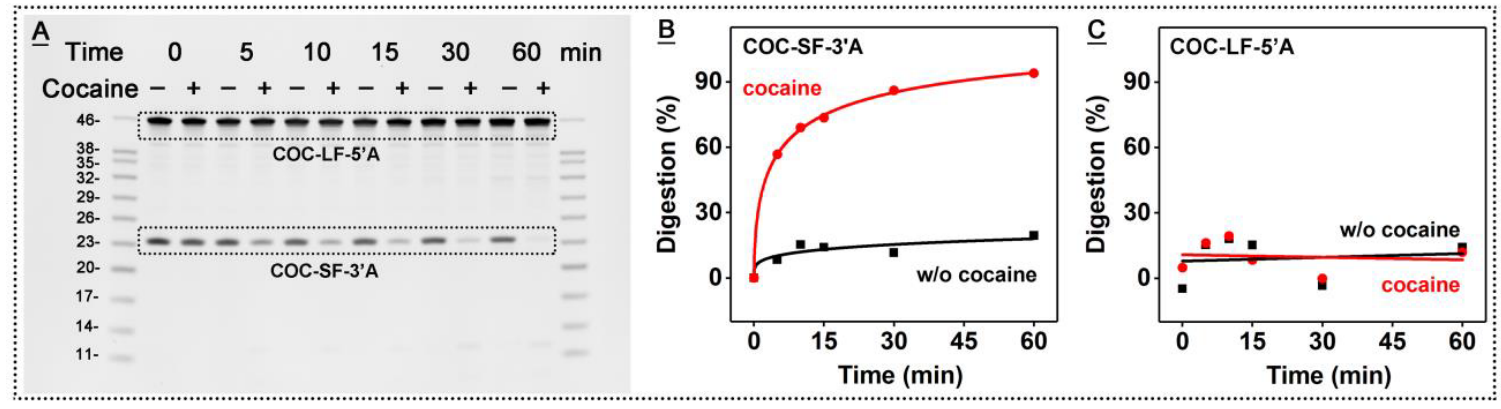

Figure 4-13. Time-course of CBSA digestion with COC-SF-3'A. (A) PAGE analysis of digestion products of CBSA consisting of $1 \mu \mathrm{M}$ COC-SF-3'A and $1 \mu \mathrm{M}$ poly(A) $)_{5}$ protected COC-LF after treatment with $0.01 \mathrm{U} / \mu \mathrm{L}$ Exo III in the presence or absence of $250 \mu \mathrm{M}$ cocaine for $5,10,15,30$ or $60 \mathrm{~min}$ of digestion. Digestion of (B) COC-SF-3'A and (C) COC-LF-5'A was quantified by $\left(\right.$ Int $_{0}$-Int $) /$ Int $_{0} \times 100 \%$, where Int is the band intensity after addition of Exo III and Int $_{0}$ is the band intensity before addition of Exo III.
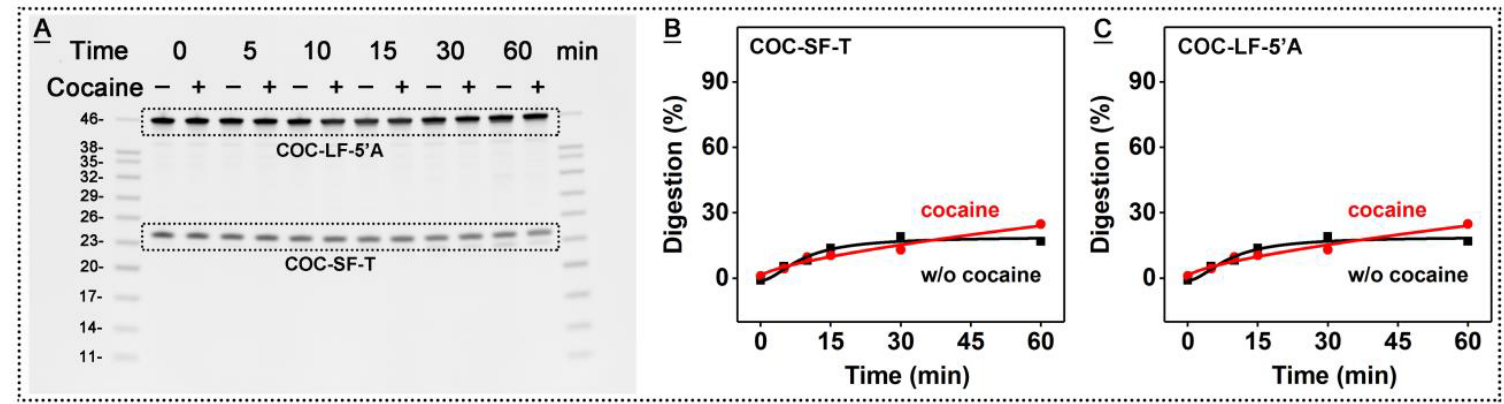

Figure 4-14. Time-course of CBSA digestion with COC-SF-T. (A) PAGE analysis of digestion products of CBSA consisting of $1 \mu \mathrm{M}$ COC-SF-T and $1 \mu \mathrm{M}$ poly(A) $)_{5}$ protected COC-LF after treatment with $0.01 \mathrm{U} / \mu \mathrm{L}$ Exo III in the presence or absence of $250 \mu \mathrm{M}$ cocaine for $5,10,15,30$ or $60 \mathrm{~min}$ of digestion. Digestion of (B) COC-SF-T and (C) COC-LF-5'A was quantified by $\left(\right.$ Int $_{0}-$ Int $_{\text {) }} /$ Int $_{0} \times 100 \%$, where Int is the band intensity after addition of Exo III and Int $_{0}$ is the band intensity before addition of Exo III.
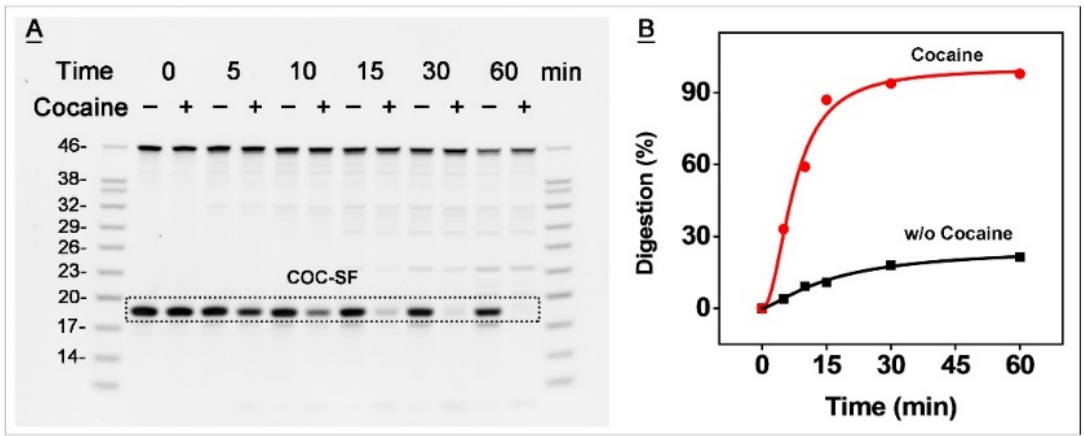

Figure 4-15. Time-course of Exo III digestion of cocaine-binding CBSA. (A) Digestion of a mixture of $1 \mu \mathrm{M}$ COC-LF and $8 \mu \mathrm{M}$ COC-SF by $0.1 \mathrm{U} / \mu \mathrm{L}$ Exo III in the presence or absence of $250 \mu \mathrm{M}$ cocaine after $5,10,15,30$, or 60 minutes of digestion. (B) Digestion was quantified by $\left(\right.$ Int $_{0}-$ Int $) /$ Int $_{0} \times 100 \%$, where Int is the band intensity after addition of Exo III and Int $_{0}$ is the band intensity before addition of Exo III. 
We then demonstrated that we could perform EATR with our cocaine-binding CBSA in solution. We performed a time-course of Exo III digestion using poly(A) 5 protected COC-LF and unprotected COC-SF, with a 1:8 ratio of COC-LF:COC-SF, either with or without $250 \mu \mathrm{M}$ cocaine. PAGE analysis of the digestion products showed rapid and specific Exo III digestion, with more than $60 \%$ of the short fragment cleaved in the presence of cocaine after only 10 minutes (Figure 4-15). In contrast, less than $5 \%$ of the short fragment was cleaved in the absence of cocaine. After a 30-minute reaction, COCSF was almost completely digested (92\%) in the presence of cocaine, while only $14 \%$ was digested in the absence of cocaine, demonstrating the specificity of EATR amplification in solution.

\subsubsection{DNA surface coverage and the length of COC-SF affect EATR-mediated}

\section{AuNP aggregation}

We employed our CBSA-based EATR-amplified assay to achieve naked-eye detection of cocaine. We conjugated a thiolated version of COC-SF (Table 4-1, SH-COC-SF-1X) onto AuNPs as described previously. ${ }^{143}$ Since DNA surface coverage has a critical influence on EATR-mediated AuNP aggregation, we developed a simple strategy for generating AuNPs displaying different quantities of short fragment. We did this by incubating short fragment-saturated AuNPs with various micromolar concentrations of DTT to displace different quantities of immobilized short fragment from the particle surface. We then measured the surface coverage resulting from treatment with various DTT concentrations by mixing each of these batches of AuNPs with an equal volume of 1.0 $\mathrm{M} \mathrm{DTT}^{128}$ and incubating overnight to remove all remaining DNA strands from the particle surface. We subsequently quantified these by centrifuging the samples and 
measured the DNA concentration in the collected supernatant with OliGreen, a DNA binding dye. ${ }^{141}$ A calibration curve (Figure 4-16A) determined that treatment with DTT concentrations of $0,100,200,300$, and $400 \mu \mathrm{M}$ produced AuNPs that respectively displayed $102 \pm 1,41 \pm 1,34 \pm 1,25 \pm 1$, and $22 \pm 1$ strands/particle (Figure 4-16B). This demonstrates that DTT treatment can effectively regulate DNA surface coverage on AuNPs in a concentration-dependent manner. AuNPs treated with $500 \mu \mathrm{M}$ DTT yielded a surface coverage of $20 \pm 1$ strands/particle; the resulting particles were unstable, and spontaneously aggregated in the reaction buffer (data not shown). Thus, these AuNPs were omitted from following experiments.
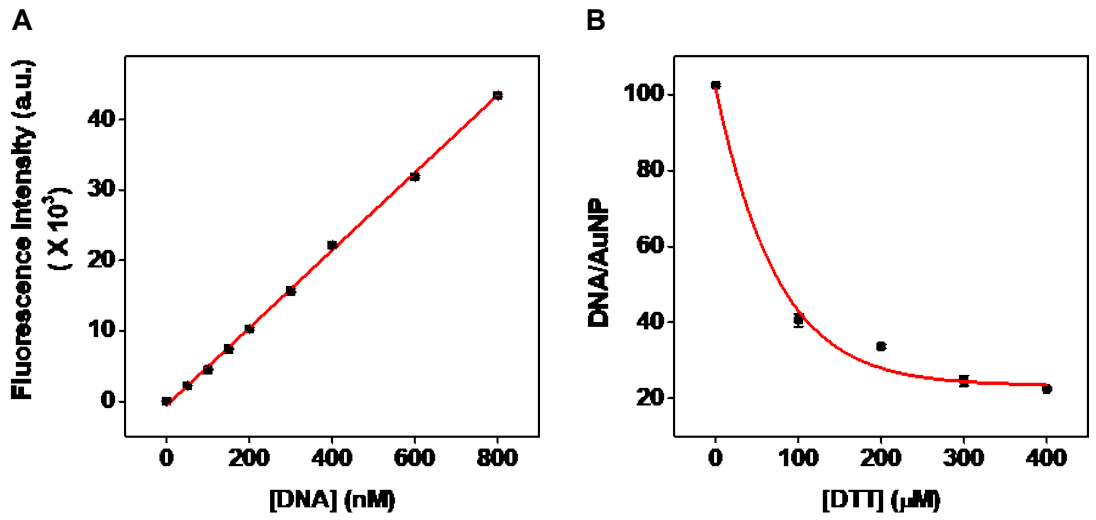

Figure 4-16. Characterizing surface coverage of SH-COC-SF-1X-modified AuNPs treated with different concentrations of DTT. (A) A standard calibration curve established with known concentrations of SH-COC-SF-1X was used to calculate the DNA surface coverage of the DTTtreated AuNPs. (B) Surface coverage decreased with increasing amounts of DTT employed during the treatment procedure. Error bars show standard deviations obtained from three measurements. 


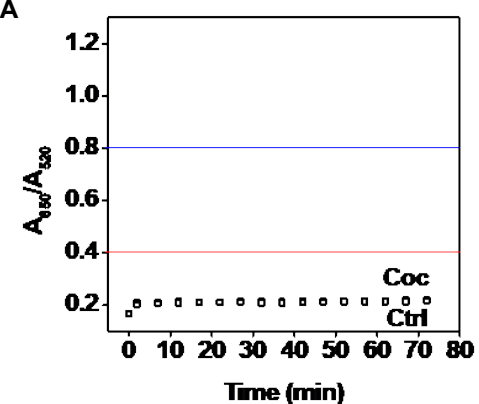

D

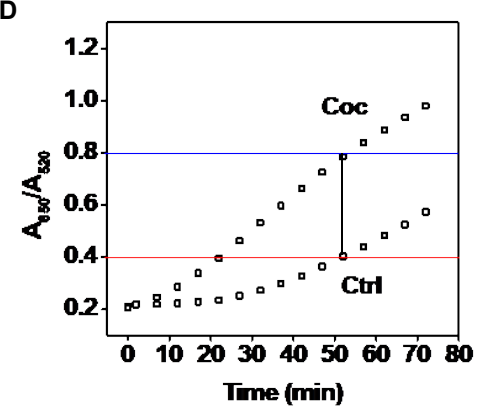

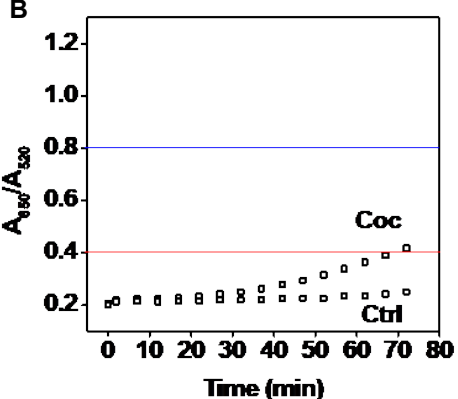

E

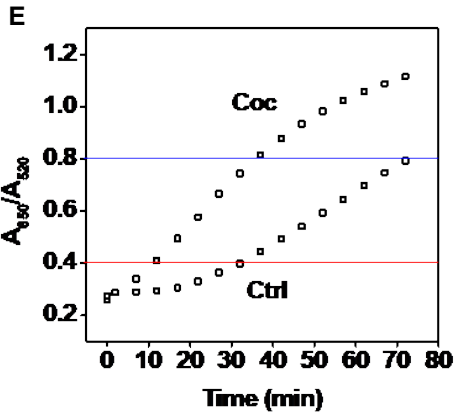

C

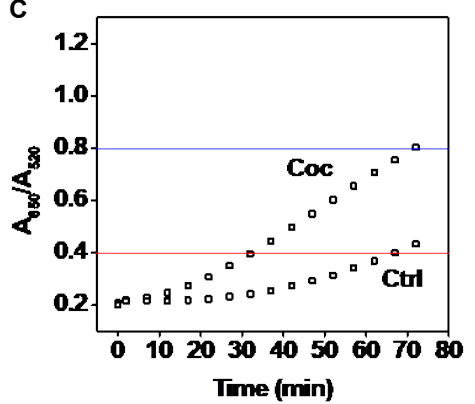

$\mathbf{F}$

Surface coverage Detection Detection (strand/particle) time window $102 \pm 1 \quad$ NA NA $41 \pm 1 \quad$ NA NA $34 \pm 1 \quad 70 \mathrm{~min} \quad \mathrm{NA}$ $25 \pm 1 \quad 52 \mathrm{~min} \quad 0.5 \mathrm{~min}$ $22 \pm 1 \quad 35 \mathrm{~min} \quad \mathrm{NA}$

Figure 4-17. Effect of SH-COC-SF-1X surface coverage. Time-dependence of Exo III digestion of AuNPs displaying SH-COC-SF-1X at (A) 102 \pm 1 , (B) 41 1 , (C) $34 \pm 1$, (D) $25 \pm 1$, or (E) $22 \pm 1$ strands/particle in the absence or presence of $250 \mu \mathrm{M}$ cocaine are shown. The detection window, during which the drug-containing sample is blue while the drug-free sample remains red, is marked as a vertical line. (F) Summary of detection time and detection window obtained using AuNPs with different surface coverages. 25 strands/particle was identified as the optimal surface coverage.

We then assessed how differing levels of surface coverage affect CBSA-cocaine assembly, exonuclease cleavage, and AuNP aggregation. Since the CBSA will be assembled on the particle, Exo III will have minimal accessibility to the 3' end of the long fragment. We incubated our various batches of $5 \mathrm{nM} \mathrm{SH-COC-SF-1X-modified}$ AuNPs with $100 \mathrm{nM}$ unprotected COC-LF in the presence or absence of $250 \mu \mathrm{M}$ cocaine for 30 minutes, and then added $0.2 \mathrm{U} / \mu \mathrm{L}$ Exo III. We tracked the signal response by performing time-dependent measurements of UV-vis absorbance. Well-dispersed AuNPs have an absorbance peak at $520 \mathrm{~nm}$; as AuNPs start to aggregate, this peak decreases and a new peak appears at $650 \mathrm{~nm} .{ }^{217}$ Therefore, we used the ratio of $\mathrm{A}_{650} / \mathrm{A}_{520}$ to evaluate EATR-mediated AuNP aggregation. When the value of $\mathrm{A}_{650} / \mathrm{A}_{520}$ is below 0.4, the 
AuNPs are well separated and the solution appears red. When the value of $A_{650} / A_{520}$ is 0.6 , the AuNPs begin to aggregate and the solution appears purple. A value of $A_{650} / A_{520} \geq$ 0.8 indicates complete aggregation, and the solution appears dark blue. We found that SH-COC-SF-1X-modified AuNPs with relatively low surface coverage (25 strands/particle) demonstrated much faster aggregation than AuNPs with high surface coverage (102 strands/particle) (Figure 4-17). We observed no aggregation for these 102 strands/particle AuNPs, even 70 minutes after the addition of Exo III (Figure 4-17A). Likewise, we did not observe a visible color change at a surface density of 41 strands/particle (Figure 4-17B). However, further reductions in surface coverage produced a clear increase in the rate of AuNP aggregation, and AuNPs with surface coverages of 34, 25 and 22 strands/particle produced a dark blue color $\left(\mathrm{A}_{650} / \mathrm{A}_{520} \geq 0.8\right)$ within 72,52 , and 38 minutes of Exo III digestion, respectively. This indicates both specific CBSA-cocaine assembly and successful EATR amplification. In the meantime, the AuNPs remained well-dispersed at all levels of surface coverage and all target-free samples remained red $\left(\mathrm{A}_{650} / \mathrm{A}_{520}=0.4\right)$ (Figure 4-17, C-E). Notably, both target- and non-target-mediated Exo III digestion and AuNP aggregation are more pronounced for AuNPs with lower surface coverages. We identified 25 strands/particle as the optimal level of surface coverage for our assay due to the existence of detection window during which the drug-containing sample is blue while the drug-free sample remains red (Figure 4-17F). Although the ratio between COC-LF and AuNP-conjugated SH-COC-SF-1X under this optimal surface coverage was close to 1:1, we believe that Exo III-mediated recycling of LF and target was taking place on the particle surface since DNA hybridization efficiency on AuNPs is usually below $20 \% .{ }^{129,136}$ We believe that our DTT- 
based strategy for regulating DNA surface coverage plays multiple roles in facilitating exonuclease digestion of AuNP-conjugated short fragments for rapid cocaine detection. First, it prevents non-specific binding of DNA bases ${ }^{218}$ by occupying vacant areas on the particle surface with DTT, lifting the covalently-bound aptamer fragments into an upright orientation that increases CBSA-target assembly and enzyme accessibility. ${ }^{219}$ Second, it adjusts the surface density by replacing some of the chemically-bound aptamer fragments. This significantly reduces the steric and electrostatic repulsion caused by neighboring DNA strands, enabling optimized CBSA-cocaine complex assembly. ${ }^{220}$ Finally, by reducing DNA surface coverage, the treatment decreases local salt concentration at the surface, ${ }^{133}$ which prevents the salt-induced inhibition of Exo III. ${ }^{216}$

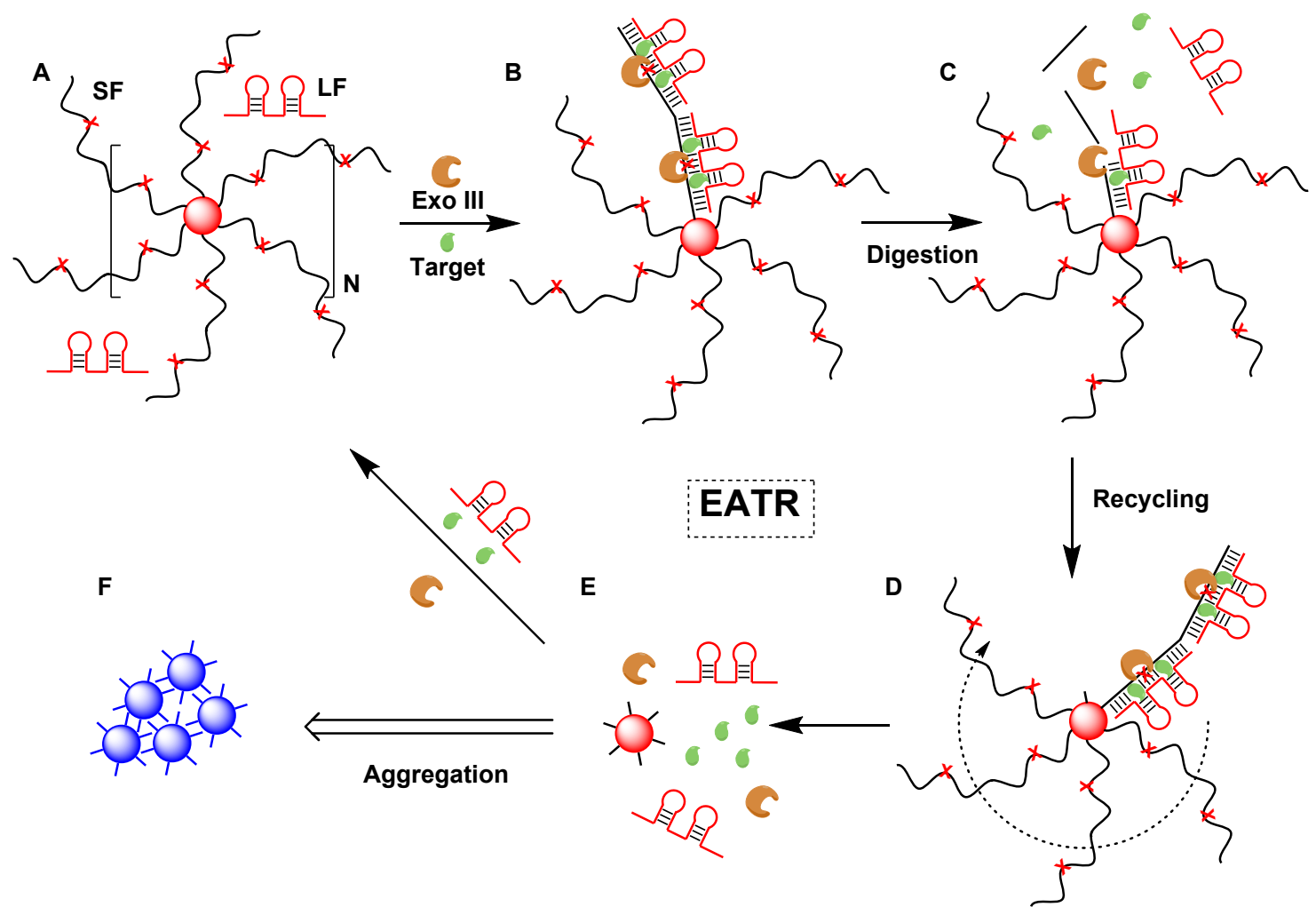

Figure 4-18. Alternative version of our colorimetric cocaine sensor using SH-COC-SF-2Xmodified AuNPs. 
A

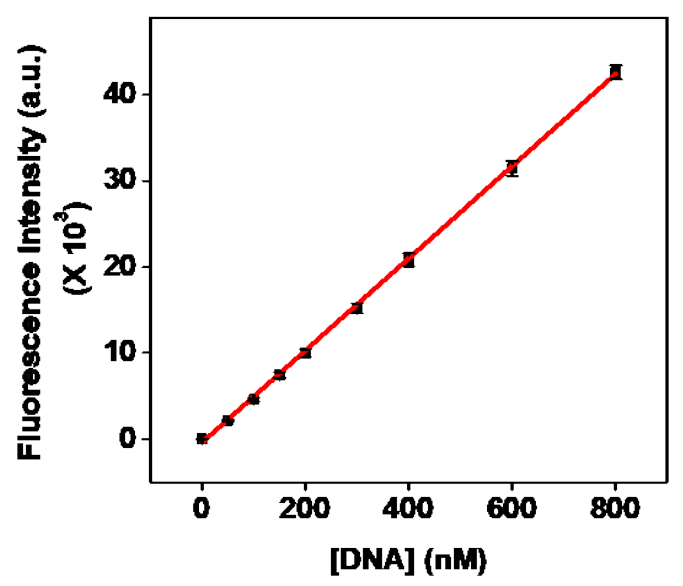

B

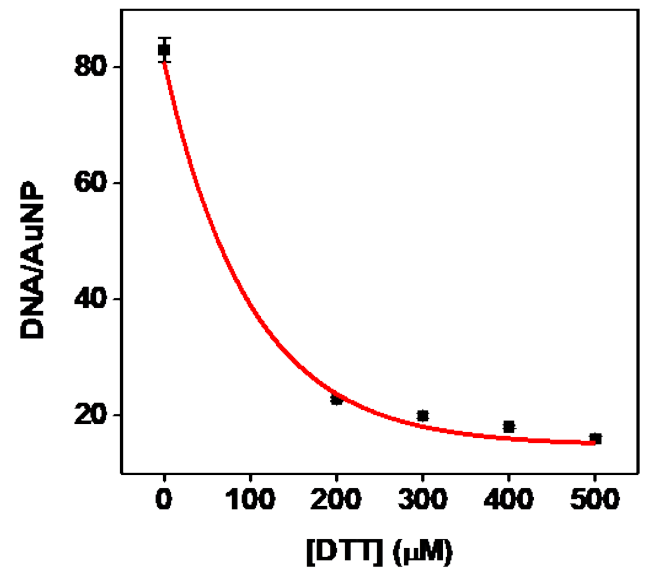

Figure 4-19. Surface coverage of SH-COC-SF-2X-modified AuNPs treated with different concentrations of DTT. (A) A calibration curve established with known concentrations of SH-COC-SF-2X was used to calculate the DNA surface coverage of the DTT-treated AuNPs. (B) The surface coverage decreased with increasing amounts of DTT employed during the treatment procedure. Error bars show standard deviations obtained from three measurements.

A

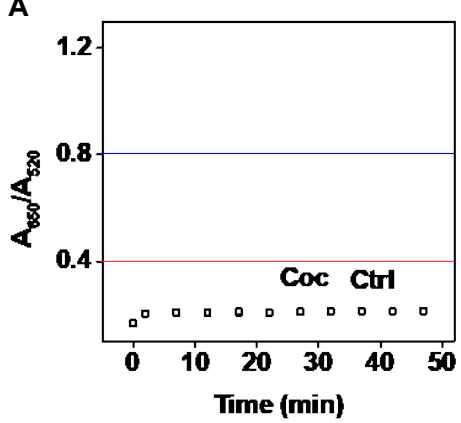

D

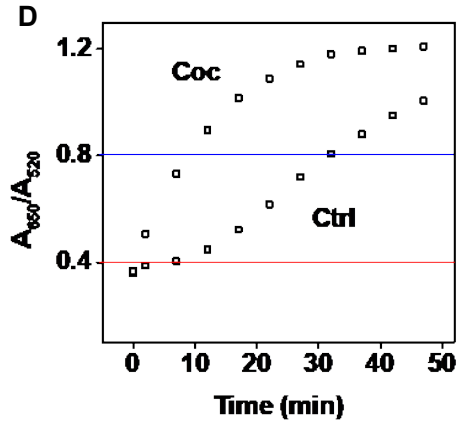

B

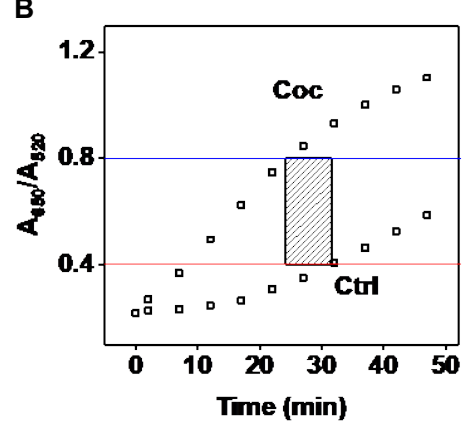

E

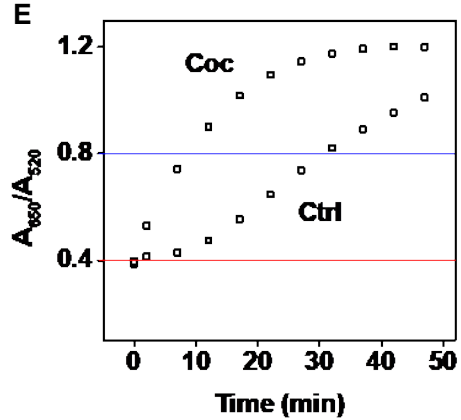

C

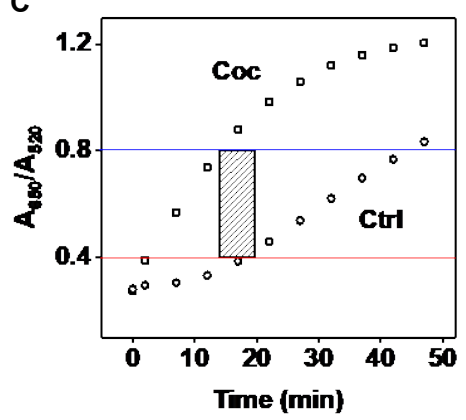

$\mathbf{F}$

\begin{tabular}{ccc}
\hline $\begin{array}{c}\text { Surface coverage } \\
\text { (strand/particle) }\end{array}$ & $\begin{array}{c}\text { Detection } \\
\text { time }\end{array}$ & $\begin{array}{c}\text { Detection } \\
\text { window }\end{array}$ \\
\hline $83 \pm 2$ & $\mathrm{NA}$ & $\mathrm{NA}$ \\
$23 \pm 1$ & $24 \mathrm{~min}$ & $8 \mathrm{~min}$ \\
$20 \pm 1$ & $14 \mathrm{~min}$ & $6 \mathrm{~min}$ \\
$18 \pm 1$ & $9 \mathrm{~min}$ & $\mathrm{NA}$ \\
$16 \pm 1$ & $9 \mathrm{~min}$ & $\mathrm{NA}$ \\
\hline
\end{tabular}

Figure 4-20. Effect of SH-COC-SF-2X surface coverage. Time-dependence of Exo III digestion of AuNPs displaying SH-COC-SF-2X at (A) $83 \pm 2$, (B) $23 \pm 1$, (C) $20 \pm 1$, (D) $18 \pm 1$, or (E) $16 \pm 1$ strands/particle in the absence or presence of $250 \mu \mathrm{M}$ cocaine are shown. The detection window, during which the drug-containing sample is blue while the drug-free sample remains red, is marked as a shaded box. (F) Summary of detection time and detection window obtained using 
AuNPs with different surface coverages. 20 strands/particle was identified as the optimal surface coverage.

We predicted that a longer version of COC-SF should further increase the stability of our modified AuNPs by suppressing nonspecific digestion-mediated aggregation. Thus, we designed a new construct (Table 4-1, SH-COC-SF-2X) comprising of two tandem abasic-site-incorporated short fragment repeats with a $(\mathrm{T})_{6}$ linker. Our reasoning was that the assay's specificity should be enhanced by this change, since Exo III will have to cleave both abasic sites in order to trigger cocaine recycling and AuNP aggregation (Figure 4-18). We modified 13-nm AuNPs with SH-COC-SF-2X, used our DTT regulation strategy to adjust the surface coverage of AuNPs, and tested the effects of DNA surface coverage on EATR performance. A calibration curve (Figure 4-19A) determined that treatment with DTT concentrations of $0,200,300,400$, and $500 \mu \mathrm{M}$ produced AuNPs that respectively displayed $83 \pm 2,23 \pm 1,20 \pm 1,18 \pm 1$, and $16 \pm 1$ strands per particle (Figure 4-19B). As expected, increasing concentrations of DTT produced AuNPs with lower levels of surface coverage (Figure 4-19). Compared to SH-COC-SF1X modified-AuNPs, SH-COC-SF-2X modified-AuNPs are stable even with surface coverages as low as 16 strands/particle. This is most likely due to the increased number of negatively charged DNA-phosphate groups located on the AuNP surface, providing more repulsion among particles. Lower-coverage AuNPs underwent EATR-mediated AuNP aggregation at a much faster rate than those with saturating coverage (Figure 4-20, A-E). The surface coverage of $20 \pm 1$ strands/particle was selected for a combination of short reaction time and long detection window (Figure 4-20F). 


\subsubsection{Visual detection of cocaine using CBSA-based EATR-amplified colorimetric assay}

Finally, we compared the assay's performance with SH-COC-SF-1X versus SH-COCSF-2X at optimized surface coverage. SH-COC-SF-2X-modified AuNPs aggregated more rapidly than those modified with SH-COC-SF-1X (Figure 4-21). We then used SHCOC-SF-2X-modified AuNPs to test the sensitivity of our assay. The particles remained stable in the reaction buffer, and the color of the sample was not affected by cocaine concentrations of up to $500 \mu \mathrm{M}$ in the absence of Exo III (Figure 4-22A, 0 min). Upon addition of Exo III, samples containing cocaine demonstrated different levels of particle aggregration, resulting in a distinctive color readout that shifted from light purple, dark purple, to dark blue at increasing drug concentrations over the course of 20 minutes (Figure 4-22A, $20 \mathrm{~min})$, with $\mathrm{A}_{650} / \mathrm{A}_{520}$ varying from $0.39(0.2-1 \mu \mathrm{M})$ to $0.90(500 \mu \mathrm{M})$. The color of the cocaine-free sample remained unchanged. We could observe a clear blue color in samples containg as little as $10 \mu \mathrm{M}$ cocaine with the naked eye after 20 minutes (Figure 4-22A). Using UV-vis spectroscopy measurements, we obtained a measurable limit of detection of $2 \mu \mathrm{M}$ (Figure 4-22B). We determined the target-specificty of the CBSA-based EATR-amplified colorimetric assay by challenging it with cocaine along with common cutting agents observed in street samples ${ }^{221}$ such as lidocaine, caffeine, levamisole, and benzocaine at a concentration of $50 \mu \mathrm{M}$. We observed a red-to-blue color change only in the cocaine sample within 20 minutes (Figure 4-23). These results show that our EATR colormietric assay can be used for the specific and sensitive naked-eye detection of cocaine in seized samples. 


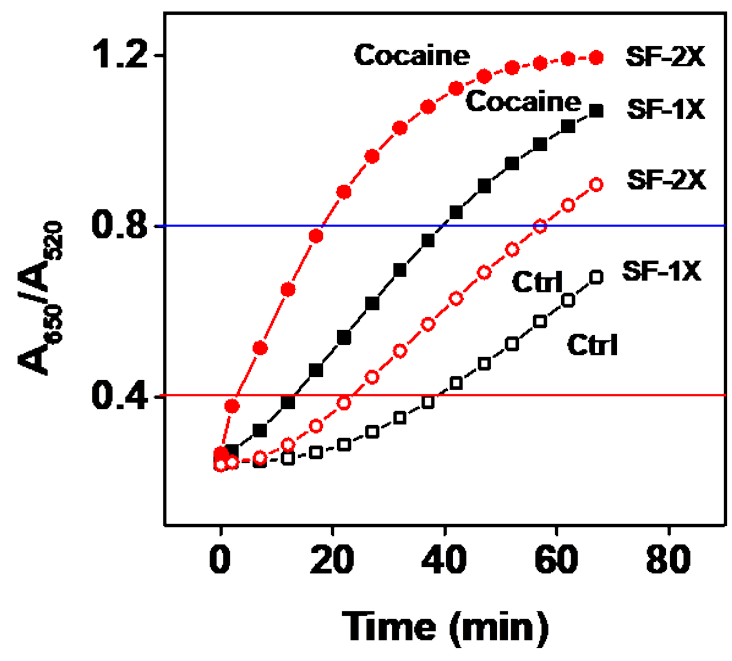

Figure 4-21. Comparison of different SF in the CBSA-based EATR-amplified colorimetric assay. Time-dependence of Exo III digestion of AuNPs modified with SH-COC-SF-1X (25 strands/particle, black) or SH-COC-SF-2X (20 strands/particle, red) in the absence (open circles and squares) or presence (solid circles and squares) of $250 \mu \mathrm{M}$ cocaine are shown.
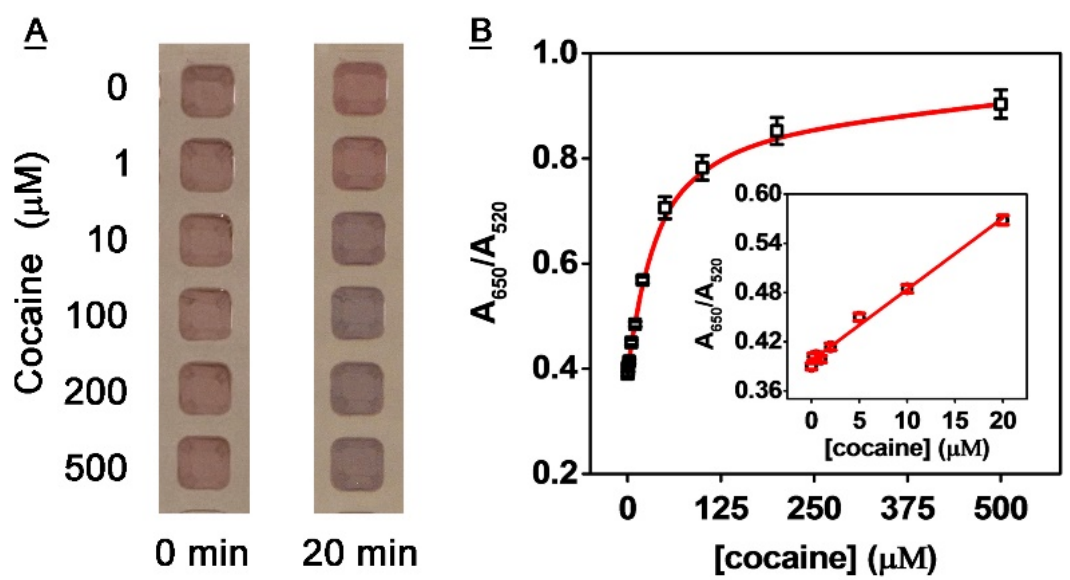

Figure 4-22. CBSA assay for cocaine detection with SH-COC-SF-2X-conjugated AuNPs. (A) After 20 minutes, samples containing cocaine at concentrations $\geq 10 \mu \mathrm{M}$ produced a clearly visible red to blue color change. Cocaine-free samples without cocaine remained red. (B) Calibration curve of our colorimetic CBSA-based cocaine assay; inset shows the calibration curve at low cocaine concentrations. 


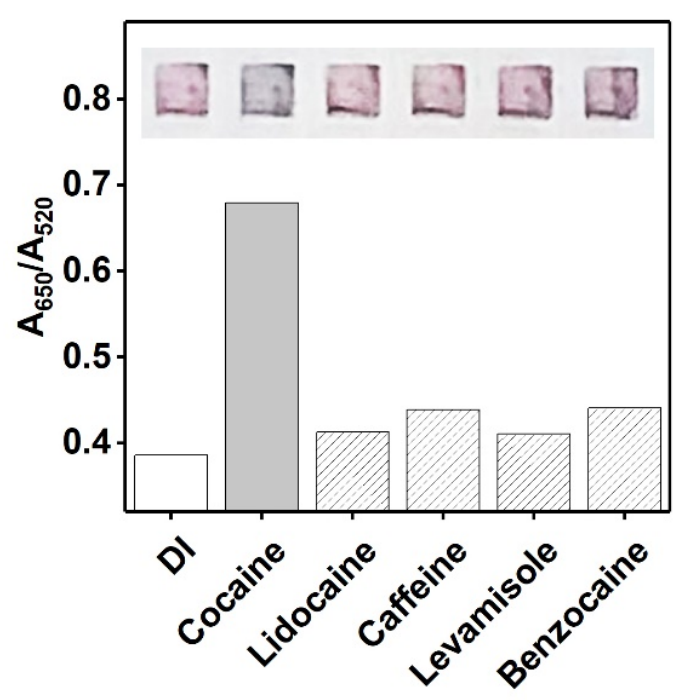

Figure 4-23. Specificity of the EATR-amplified CBSA-based colorimetric assay for $50 \mu \mathrm{M}$ cocaine versus various interferents. A red-to-blue color change was observed in the cocaine sample, whereas lidocaine, caffeine, levamisole, and benzocaine samples remained red after 20 minutes of digestion.

\subsection{Conclusions}

We have demonstrated a general and simple approach for developing a variety of CBSA-based EATR-amplified assays for the sensitive detection of small-molecule targets. The 3'-5' exonuclease activity of Exo III has been used extensively in DNAbased EATR assays, ${ }^{159}$ but our results demonstrated that Exo III's 3'-5' exonuclease activity is greatly inhibited by the tertiary structure of target-CBSA complexes. To achieve rapid CBSA-based EATR, we therefore incorporated a C3 spacer abasic site between the two target-binding domains on our CBSA short fragment, creating a recognition site for Exo III's apurinic endonuclease activity. We have previously demonstrated that the apurinic endonuclease activity of Exo III can effectively enable EATR-mediated AuNP aggregation for rapid and sensitive detection of single-base mismatched DNA. ${ }^{154}$ Here, we showed that Exo III's apurinic endonuclease activity could mediate CBSA-based EATR in solution as well as on particle surface. We thus 
believe that the highly active and robust apurinic endonuclease activity of Exo III should allow many DNA-based EATR sensor platforms to be applied to our CBSA-based EATR assays for detection of various small-molecule targets.

As an initial demonstration, we engineered a DIS-binding CBSA with a duplexed C3 spacer abasic site incorporated between the two target-binding domains. This allowed us to exploit EATR signal amplification, which enhanced the sensitivity of our CBSA-based fluorescence assay by 100-fold relative to an unamplified assay. The detection limit of our CBSA-based EATR-amplified fluorescence assay is 100 and 50 times lower than previously reported DIS assays based on a split aptamer ${ }^{92}$ and a structure-switching aptamer, ${ }^{222}$ respectively. This assay also allowed us to detect DIS at concentrations as low as $1 \mu \mathrm{M}$ in $50 \%$ urine within 30 minutes. We then demonstrated that this strategy could be adopted for rapid colorimetric detection of cocaine, based on EATR-mediated aggregation of AuNPs - a useful strategy for instrument-free detection. This assay enabled us to achieve rapid, naked-eye detection of cocaine at low micromolar concentrations within 20 minutes and is more sensitive, robust, or simple compared to previously reported methods for the visual detection of cocaine (Table 4-2).

Recent work has described SELEX approaches for isolating small molecule-binding aptamers with a three-way junction structure using a partially-structured library. ${ }^{33}$ These aptamers can be readily engineered into CBSAs using the strategy demonstrated in this work and, as also shown here, these CBSAs can then be readily adapted for use in EATRamplified assays. Based on the generality of our CBSA engineering procedure and excellent performance of EATR assays in complex samples, we believe that the strategy described here will accelerate the development of sensitive and accurate sensors for 
detecting small-molecule targets in fields including environmental monitoring, food safety, law enforcement, medical diagnostics, and public health.

Table 4-2. Comparison of assays for the visual detection of cocaine.

\begin{tabular}{|c|c|c|c|c|}
\hline Detection strategy & $\begin{array}{l}\text { Visual } \\
\text { Detection } \\
\text { limit }\end{array}$ & $\begin{array}{l}\text { Detection } \\
\text { Time }\end{array}$ & Existing problems & Ref \\
\hline $\begin{array}{l}\text { CBSA-based EATR-amplified } \\
\text { assay }\end{array}$ & $10 \mu \mathrm{M}$ & $20 \mathrm{~min}$ & Requires enzyme reaction & $\begin{array}{l}\text { This } \\
\text { work }\end{array}$ \\
\hline $\begin{array}{l}\text { Dissociation of aptamer- } \\
\text { hybridized DNA-conjugated } \\
\text { AuNP-assemblies }\end{array}$ & $500 \mu \mathrm{M}$ & $1 \mathrm{~min}$ & $\begin{array}{l}\text { Preparation of AuNP } \\
\text { assemblies is laborious }\end{array}$ & 144 \\
\hline Dye-displacement assay & $500 \mu \mathrm{M}$ & Overnight & Long reaction time & 101 \\
\hline $\begin{array}{l}\text { Aggregation of split aptamer- } \\
\text { protected unmodified AuNPs }\end{array}$ & $50 \mu \mathrm{M}$ & $3 \mathrm{~min}$ & $\begin{array}{l}\text { Unmodified AuNPs are } \\
\text { unstable }\end{array}$ & 145 \\
\hline $\begin{array}{l}\text { AuNP and conjugated } \\
\text { polyelectrolyte }\end{array}$ & $50 \mu \mathrm{M}$ & $2 \mathrm{~min}$ & $\begin{array}{l}\text { Unmodified AuNPs are } \\
\text { unstable }\end{array}$ & 146 \\
\hline $\begin{array}{l}\text { Target-induced dissociation of } \\
\text { aptamer-linked hydrogels }\end{array}$ & $2 \mu \mathrm{M}$ & $10 \mathrm{~min}$ & $\begin{array}{l}\text { Enzyme-embedded hydrogel } \\
\text { is hard to preserve }\end{array}$ & 207 \\
\hline
\end{tabular}


CHAPTER 5: In Vitro Isolation of Small-Molecule-Binding Aptamers with Intrinsic

Dye-Displacement Functionality

\subsection{Introduction}

Aptamers are nucleic acid-based molecules that can be isolated in vitro through SELEX techniques to bind various targets with high specificity and affinity. ${ }^{1,5}$ They are increasingly being used as recognition elements in biosensing platforms due to their low cost of production, ease of modification, chemical stability, and long shelf life. ${ }^{5,76,77}$ The generation of a sensor readout typically requires an aptamer to undergo some manner of binding-induced change, and most aptamer-based sensors utilize structure-switching aptamers that undergo a major conformational rearrangement upon target binding. However, most aptamers do not innately undergo a measurable binding-induced conformational change, and the development of a structure-switching aptamer typically entails a multi-stage process of sequence analysis and chemical modification. ${ }^{75-77}$

SELEX methods have been developed that make it possible to isolate aptamers with inherent structure-switching functionality. For example, Ellington ${ }^{98}$ and $\mathrm{Li}^{32}$ have utilized a 'strand-displacement' strategy to directly isolate structure-switching aptamers. Their approach begins with the hybridization of library molecules with an immobilized complementary strand. Library strands that bind to the target undergo a conformational change, dissociate from the complementary strand, and are collected in the supernatant. After several rounds of isolation and enrichment, these aptamers are sequenced, chemically modified with a fluorophore, and can then be directly employed in stranddisplacement assays with a quencher-modified complementary strand. This approach eliminates the need for sequence engineering, but there is an inherent conflict between 
the requirements for aptamer isolation and sensor development. This is because the isolation of high-affinity aptamers requires strong hybridization of the complementary strand with the library molecules in order to create an energetic barrier for stringent selection. Nevertheless, since competitive strands have high binding affinity for the aptamer, they inadvertently inhibit target binding and consequently yielding low assay sensitivity. ${ }^{32,33,97,99,100}$ Moreover, strand-displacement assays typically require a lengthy heating-and-cooling process to hybridize the complementary strand with the aptamer, which greatly hinders the use of these assays for rapid on-site detection. Nonetheless, the strand-displacement assay is presently the only generally applicable method for developing aptamer-based small-molecule sensors directly from isolated aptamers without further engineering.

Dye-displacement strategies offer an appealing alternative in this regard. ${ }^{101-104}$ In such assays, a small-molecule dye competes with the target to bind with the aptamer. Displacement of the dye from the aptamer's target-binding domain results in a change of the dye absorbance or fluorescence. Such assays not only require no chemical modification of the aptamer but also yield high sensitivity, since displacement of a smallmolecule dye is more thermodynamically feasible compare to a tightly-bound complementary strand. However, this assay strategy has not been generalized, as there is currently no technology to generate aptamers with dye-displacement functionality.

Herein, we for the first time have developed a new library-immobilized SELEX strategy for the isolation of small-molecule-binding aptamers with inherent dyedisplacement functionality. The isolated aptamers can rapidly report binding events through target-induced dye-displacement, which can then be employed in a generalized 
sensor platform for the detection of small-molecule targets. Previous research has demonstrated that diethylthiatricarbocyanine (Cy7) can bind to a cocaine-binding aptamer via its three-way junction (TWJ) binding domain, ${ }^{101}$ and we have now demonstrated that such $\mathrm{Cy} 7$ binding is a general and sequence-independent feature of DNA TWJ structures. This interaction produces a distinctive change in absorbance at 670 $\mathrm{nm}$ and $760 \mathrm{~nm}$, which allowed us to isolate aptamers that undergo target bindinginduced dye-displacement from a TWJ-containing structured library. These aptamers can then be directly employed to optically report the presence of their respective target through displacement of Cy7. As a demonstration, we isolated an aptamer for 3,4methylenedioxypyrovalerone (MDPV), a synthetic cathinone designer drug. The isolated aptamer retains a TWJ-structured binding domain and has a $\mathrm{K}_{\mathrm{D}}$ of $6.1 \mu \mathrm{M}$. The addition of MDPV displaced Cy7 from the TWJ domain, resulting in a change in the absorbance of the dye. Using this label-free assay, we were able to detect MDPV at a concentration as low as $300 \mathrm{nM}-20$-fold lower than the $\mathrm{K}_{\mathrm{D}}$ of the aptamer - within seconds. Our Cy7displacement assay was more sensitive and rapid than an equivalent strand-displacement fluorescence assay, which used a fluorophore-modified version of the same aptamer along with a quencher-modified complementary strand. Importantly, our isolated aptamer is also cross-reactive to other synthetic cathinones, which represent a diverse emerging class of drugs of abuse, while remaining non-responsive to other structurally-similar noncathinone molecules and commonly-used cutting agents. Our method should be generally applicable as a means to enable the rapid and sensitive detection of a variety of smallmolecule targets that bind to three-way junction-derived receptors. 


\subsection{Experimental section}

\subsubsection{Materials}

Table 5-1. The sequences of DNA oligonucleotides employed in Chapter 5.

\begin{tabular}{ll}
\hline Sequence ID & Sequence $\left(5^{\prime}-3^{\prime}\right)$ \\
\hline DISS.1 & CTCGGGACGTGGATTTCCGCATACGAAGTTGTCCCGAG \\
DOGS.1 & TCGGGACGTGGATTTTCCACAAACCAGAATGGTGTCCCGA \\
TWJ pool & CTTACGACNNNGGCATTTTGCCNNNNAACGAAGTTNNNGTCGTAAG \\
Library & CGAGCATAGGCAGAACTTACGACNNNGGNATTTTNCCNNNNANCG \\
S1 stem-loop & CATAATNNNGTCGTAAGAGCGAGTCATTC \\
S2 stem-loop & GGCATTTTGCC \\
S3 stem-loop & AACGAAGTT \\
cDNA-bio & TTTTTGTCGTAAGTTCTGCCATTTT/Bio/ \\
FP & GCAGCATAGGCAGAACTTAC \\
RP & GAATGACTCGCTCTTACGAC \\
RP-bio & /Bio/GAATGACTCGCTCTTACGAC \\
MA & CTTACGACTCAGGCATTTTGCCGGGTAACGAAGTTACTGTCGTAAG \\
MA-L & CAGAACTTACGACTCAGGCATTTTGCCGGGTAACGAAGTTACTGTC \\
MA-F & GTAAG \\
cDNA & ACTG/GGCAGAACTTACGACTCAGGCATTTTGCCGGGTAACGAAGTT \\
cDNA-Q & GTCGTAAGTTCTGCC \\
\hline \hline
\end{tabular}

d. $N$ represents random base

e. /Bio/ represents biotin modification

f. $/ 5 \mathrm{Cy} 5 /$ represents $\mathrm{Cy} 5$ fluorophore modification

g. /3IAbRQsp/ represents Iowa Black RQ quencher modification

All oligonucleotides were ordered from Integrated DNA Technologies (IDT), purified with HPLC, and dissolved in PCR quality water (Invitrogen). DNA concentrations were measured using a NanoDrop 2000 spectrophotometer (Thermo Scientific). Cy7, dehydroisoandrosterone-3-sulfate (DIS) sodium salt dihydrate, deoxycorticosterone-21 glucoside (DOG) and all other chemicals were purchased from Sigma-Aldrich unless otherwise noted. Drug standards, including MDPV, mephedrone, methylone, naphyrone, 
and pentylone were purchased from Cayman Chemicals. Tween 20 and formamide were purchased from Fisher Scientific. Streptavidin-coated agarose resin (1-3 mg biotinylated $\mathrm{BSA} / \mathrm{ml}$ resin), One Shot Chemically Competent $E$. coli, and SYBR Gold were purchased from Thermo Scientific. $500 \mu \mathrm{L}$ micro-gravity columns were purchased from BioRad.

\subsubsection{SELEX procedure}

The isolation of MDPV-binding aptamers follows a previously-reported SELEX method $^{33}$ with some modifications. Briefly, during each round of SELEX, 100 pmole of either the TWJ-containing library or the enriched pool from a given round was mixed with 500 pmole of biotinylated complementary strand (cDNA-bio) in $250 \mu \mathrm{L}$ selection buffer (10 mM Tris-HCl, $0.5 \mathrm{mM} \mathrm{MgCl} 2,20 \mathrm{mM} \mathrm{NaCl}, \mathrm{pH} 7.4)$. The mixture was heated at $95{ }^{\circ} \mathrm{C}$ for $5 \mathrm{~min}$, slowly cooled to room temperature over $30 \mathrm{~min}$, and then loaded into a micro-gravity column containing $250 \mu \mathrm{L}$ of streptavidin-coated agarose resin for library immobilization. Unconjugated library and complementary strands were removed by flowing $250 \mu \mathrm{L}$ of selection buffer through the library-immobilized column at least 10 times. Target-bound aptamers were then eluted at room temperature with $250 \mu \mathrm{L}$ of selection buffer containing either $100 \mu \mathrm{M}$ (Rounds 1-5) or $50 \mu \mathrm{M}$ (Rounds 6-10) MDPV. Each elution was completed in two minutes. The elution was repeated two more times to increase the yield. The combined eluted aptamers were concentrated using a $3 \mathrm{~K}$ molecular weight cut-off spin filter (Millipore) and mixed with $1 \mu \mathrm{M}$ forward primer (Table 5-1, FP) and $1 \mu \mathrm{M}$ biotinylated reverse primer (Table 5-1, RP-bio) in $1 \mathrm{~mL}$ GoTaq Hot Start Master Mix (Promega). Polymerase chain reaction (PCR) was then performed using a BioRad C1000 thermal cycler. Amplification conditions were as follows: 1 cycle of $95{ }^{\circ} \mathrm{C}$ for $2 \mathrm{~min} ; 13$ cycles of $95^{\circ} \mathrm{C}$ for $15 \mathrm{~s}, 58^{\circ} \mathrm{C}$ for $30 \mathrm{~s}$, and $72{ }^{\circ} \mathrm{C}$ for $45 \mathrm{~s}$; and 1 
cycle of $72{ }^{\circ} \mathrm{C}$ for $2 \mathrm{~min}$. The double-stranded PCR product was immobilized on a microgravity column containing $200 \mu \mathrm{L}$ of streptavidin-coated agarose resin. The column was washed five times with $200 \mu \mathrm{L}$ of $10 \mathrm{mM}$ Tris- $\mathrm{HCl}$ buffer (pH 7.4) containing $20 \mathrm{mM}$ $\mathrm{NaCl}$ to remove unconjugated strands, and then incubated with $300 \mu \mathrm{L}$ of $0.2 \mathrm{M} \mathrm{NaOH}$ for $10 \mathrm{~min}$. Finally, the eluent was collected and neutralized with $0.2 \mathrm{M} \mathrm{HCl}$ in a $1.5 \mathrm{~mL}$ microcentrifugation tube and concentrated using a $3 \mathrm{~K}$ molecular weight cut-off spin filter. The enriched pool was used for the next round of SELEX after measuring its concentration with a NanoDrop 2000.

Counter-SELEX was performed against methamphetamine (Rounds 4-5), amphetamine (Rounds 6-7) and dopamine (Rounds 8-10). Specifically, the libraryimmobilized column was washed 10 times with $250 \mu \mathrm{L}$ of $100 \mu \mathrm{M}$ counter-SELEX target in selection buffer, followed by 20 times with $250 \mu \mathrm{L}$ of selection buffer prior to target elution.

\subsubsection{Cloning and sequencing}

After 10 rounds of SELEX, the final enriched pool was PCR amplified with unlabeled forward and reverse primers (Table 5-1, FP and RP) under the conditions described above, with a prolonged 30 -min extension step at $72{ }^{\circ} \mathrm{C}$ to add an A-tail. The PCR product was cloned into E. coli using the TOPO TA cloning kit (Invitrogen). 50 colonies were randomly picked and sequenced at the Florida International University DNA Core Facility. Multiple sequence alignments were carried out using BioEdit software, and the sequence logo was generated using WebLogo. ${ }^{223}$ 


\subsubsection{Characterization of target displacement of $\mathrm{Cy} 7$ from TWJ-structured aptamers}

$5 \mu \mathrm{L}$ of DISS. 1 aptamer or DOGS.1 aptamer (1) (final concentration: $7 \mu \mathrm{M}$ ), $5 \mu \mathrm{L}$ of Cy7 (final concentration: $3 \mu \mathrm{M}$ ), $5 \mu \mathrm{L}$ of DIS or DOG (final concentration: 0 or $250 \mu \mathrm{M}$ ), and $35 \mu \mathrm{L}$ selection buffer (1) $(20 \mathrm{mM}$ Tris- $\mathrm{HCl}, 10 \mathrm{mM} \mathrm{MgCl} 2,1 \mathrm{M} \mathrm{NaCl}, 0.01 \%$ Tween 20, 1\% DMSO, pH 7.4) were mixed in wells of a 384-well plate. UV-vis spectra were immediately recorded from 450-900 nm using a Tecan Infinite M1000 PRO at room temperature.

\subsubsection{Characterization of binding of Cy7 to TWJ pool}

$5 \mu \mathrm{L}$ of different concentrations of TWJ pool, $5 \mu \mathrm{L}$ of $20 \mu \mathrm{M} \mathrm{Cy}$, and $40 \mu \mathrm{L}$ of reaction buffer (final concentration $10 \mathrm{mM}$ Tris- $\mathrm{HCl}$, $0.5 \mathrm{mM} \mathrm{MgCl}, 20 \mathrm{mM} \mathrm{NaCl}$, $0.01 \%$ Tween 20 , and 1\% DMSO, pH 7.4) were mixed in wells of a 384-well plate. UVvis spectra were immediately recorded from $450 \mathrm{~nm}$ to $900 \mathrm{~nm}$ at room temperature. The absorbance value at $760 \mathrm{~nm}$ was plotted against the concentration of added TWJ pool. The $\mathrm{K}_{\mathrm{D}}$ was estimated by non-linear fitting using the Langmuir equation.

\subsubsection{Continuous variation experiment (Job plot)}

$10 \mu \mathrm{M}$ Cy7 and $10 \mu \mathrm{M}$ TWJ pool were separately prepared in reaction buffer. Different ratios of Cy7 and TWJ pool were then mixed, with the concentration of both species totaling $10 \mu \mathrm{M} .50 \mu \mathrm{L}$ of each mixture was loaded into a 384-well plate, and UVvis spectra were immediately recorded from $450-900 \mathrm{~nm}$ at room temperature. The absorbance at $760 \mathrm{~nm}$ was plotted versus the mole fraction of $\mathrm{Cy} 7$. 


\subsubsection{Development of a target elution assay to assess pool affinity and target}

specificity

After each round of SELEX, 50 pmole of the enriched pool was mixed with 250 pmole of biotinylated complementary strand (Table 5-1, cDNA-bio) in $125 \mu \mathrm{L}$ binding buffer (10 mM Tris-HCl, $0.5 \mathrm{mM} \mathrm{MgCl} 2,20 \mathrm{mM} \mathrm{NaCl}, 0.01 \%$ Tween 20\%, $\mathrm{pH} 7.4$ ). The mixture was heated to $95{ }^{\circ} \mathrm{C}$ for $5 \mathrm{~min}$, slowly cooled down to room temperature over 30 min, and loaded into a micro-gravity column containing $125 \mu \mathrm{L}$ of streptavidin-coated agarose resin for library immobilization. The library-immobilized resin was collected into a microcentrifugation tube and washed five times with $625 \mu \mathrm{L}$ binding buffer on an endto-end rotator to remove unconjugated library and complementary stands. After washing, the library-conjugated resin was re-suspended in $150 \mu \mathrm{L}$ binding buffer and aliquoted into six PCR tubes (20 $\mu \mathrm{L}$ each). $50 \mu \mathrm{L}$ binding buffer containing different amounts of MDPV was added to these tubes to reach final target concentrations of $0,10,50,100$, 250 , and $500 \mu \mathrm{M}$. After a 60 -min incubation on an end-over-end rotator, the agarose resin was precipitated by centrifugation. $3 \mu \mathrm{L}$ of supernatant was collected and mixed with 6 $\mu \mathrm{L}$ of gel loading buffer (75\% formamide, 10\% glycerol, $0.125 \%$ SDS, 10 mM EDTA, and $0.15 \%(\mathrm{w} / \mathrm{v})$ xylene cyanol). $5 \mu \mathrm{L}$ of each collected sample was then loaded into the wells of a $15 \%$ denaturing polyacrylamide gel. A control sample (40 nM initial random library) was also loaded into the gel to evaluate the concentration of target-eluted library in each sample. Separation was carried out at $20 \mathrm{~V} / \mathrm{cm}$ for 1 hour in $0.5 \times \mathrm{TBE}$ running buffer. The gel was stained with $1 \times$ SYBR Gold solution for $25 \mathrm{~min}$ and imaged using a ChemiDoc MP Image System (Bio-Rad). The percentages of elution were calculated from the concentration of target-eluted library and the initial amount of library used in 
immobilization, which were then plotted versus the concentration of MDPV. $K_{D}$ of the library pool was estimated with non-linear curve fitting using the Langmuir equation. The target specificity for the enriched pool was tested using the method described above, by eluting library-immobilized agarose resin with binding buffer, $500 \mu \mathrm{M}$ MDPV, $500 \mu \mathrm{M}$ methamphetamine, $500 \mu \mathrm{M}$ amphetamine and $500 \mu \mathrm{M}$ dopamine.

\subsubsection{Isothermal titration calorimetry (ITC)}

All ITC experiments were performed at $23{ }^{\circ} \mathrm{C}$ in reaction buffer with a MicroCal iTC200 instrument (Malvern). The sample cell contained $20 \mu \mathrm{M}$ MA, $20 \mu \mathrm{M}$ MA-L, 20 $\mu \mathrm{M}$ Cy7-MA complex (1:1), $20 \mu \mathrm{M}$ Cy7:MA-L complex (1:1), or $20 \mu \mathrm{M}$ cDNA:MA-L complex (1:1). The syringe contained $1 \mathrm{mM}$ MDPV in reaction buffer. Each experiment consisted of 19 successive $2 \mu \mathrm{L}$ injections after a $0.4 \mu \mathrm{L}$ purge injection with spacing of 180 seconds to a final molar ratio of 11:1 (MDPV:Aptamer). The raw data were first corrected based on the heat of dilution of target, and then analyzed with the MicroCal analysis kit integrated into Origin 7 software with a single-site binding model.

\subsubsection{Strand-displacement fluorescence assay for detection of MDPV}

First, the molar ratio between the fluorophore-labeled aptamer strand (Table 5-1, MAF) and the quencher-labeled complementary strand (Table 5-1, cDNA-Q) was optimized. Specifically, $8 \mu \mathrm{L}$ of MA-F (final concentration $50 \mathrm{nM}$ ) and $8 \mu \mathrm{L}$ of different concentrations of cDNA-Q were mixed with reaction buffer to a total volume of $80 \mu \mathrm{L}$. The samples were then heated at $95{ }^{\circ} \mathrm{C}$ for 5 min and slowly cooled down to room temperature over $30 \mathrm{~min} .75 \mu \mathrm{L}$ of samples were loaded into a 384 -well plate, and the

fluorescence spectra were recorded from $655-850 \mathrm{~nm}$ with excitation at $648 \mathrm{~nm}$ at room temperature. To perform the strand-displacement fluorescence assay, reaction buffer 
containing $50 \mathrm{nM}$ MA-F and $50 \mathrm{nM}$ cDNA-Q (final concentration) was heated at $95{ }^{\circ} \mathrm{C}$ for $5 \mathrm{~min}$ and slowly cooled down to room temperature over $30 \mathrm{~min} .8 \mu \mathrm{L}$ of different concentrations of MDPV were then mixed with $72 \mu \mathrm{L}$ of the cDNA-Q:MA-F complex in the wells of a 384-well plate. After 10 min of incubation, fluorescence spectra were recorded from $655-850 \mathrm{~nm}$ with excitation at $648 \mathrm{~nm}$ at room temperature. The signal gain was calculated by $\left(\mathrm{F}-\mathrm{F}_{0}\right) / \mathrm{F}_{0}$, where $\mathrm{F}_{0}$ and $\mathrm{F}$ are the fluorescence intensity at $668 \mathrm{~nm}$ without and with MDPV, respectively.

\subsubsection{Cy7 displacement assay for detection of MDPV}

$5 \mu \mathrm{L}$ of MDPV-binding aptamer (Table 5-1, MA) (final concentration: $7 \mu \mathrm{M}$ ), $5 \mu \mathrm{L}$ of Cy7 (final concentration $=3 \mu \mathrm{M}$ ), $5 \mu \mathrm{L}$ of varying concentrations of MDPV, and $35 \mu \mathrm{L}$ of reaction buffer (10 mM Tris- $\mathrm{HCl}, 0.5 \mathrm{mM} \mathrm{MgCl}_{2}, 20 \mathrm{mM} \mathrm{NaCl}, 0.01 \%$ Tween 20, $1 \%$ DMSO, pH 7.4) were mixed in wells of a 384-well plate. UV-vis spectra were immediately recorded from 450-900 nm using a Tecan Infinite M1000 PRO at room temperature. Signal gain was calculated by $\left(\mathrm{R}-\mathrm{R}_{0}\right) / \mathrm{R}_{0}$, where $\mathrm{R}_{0}$ and $\mathrm{R}$ are the $670 / 760$ $\mathrm{nm}$ absorbance ratio without and with MDPV, respectively. The same assay was performed with $50 \mu \mathrm{M}$ and $250 \mu \mathrm{M}$ of naphyrone, methylone, pentylone and mephedrone to evaluate the cross-reactivity of the assay. Additionally, we tested the specificity of the assay using $50 \mu \mathrm{M}$ and $250 \mu \mathrm{M}$ of interfering agents such as amphetamine, methamphetamine, dopamine, lidocaine, benzocaine, and caffeine.

\subsection{Results and discussion}

\subsubsection{Binding mechanism of Cy7 to TWJs}

Stojanovic et al. have previously reported that Cy7 (Figure 5-1A) specifically binds to the TWJ-structured binding domain of a cocaine-binding aptamer via hydrophobic 
interactions. ${ }^{101}$ Cocaine displaces $\mathrm{Cy} 7$ from the TWJ, resulting in a significant reduction in the absorbance of the dye at $760 \mathrm{~nm}$. We demonstrated the generality of this Cy7displacement mechanism using another two reported TWJ-structured aptamers which bind to DIS and DOG. ${ }^{33}$ Cy7 was immediately displaced from both aptamers upon the addition of their respective targets, yielding a significant decrease in the absorbance of the dye at $760 \mathrm{~nm}$ (Figure 5-2). Based on these results, we hypothesized that $\mathrm{Cy} 7$ binds to our TWJs in a sequence-independent manner, a principle that could guide the SELEXbased isolation of new small-molecule binding aptamers with intrinsic Cy7-displacement functionality.

To confirm our hypothesis, we chemically synthesized a 46-nt TWJ-structured DNA library, in which each strand contains three fully complementary stems (S1, S2, and S3) and 10 randomized nucleotides within the junction itself (Figure 5-1A and Table 5-1, TWJ pool). After mixing $2 \mu \mathrm{M} \mathrm{Cy} 7$ with $10 \mu \mathrm{M}$ TWJ pool, we observed an increase in the absorbance of the dye at $760 \mathrm{~nm}$ and $670 \mathrm{~nm}$, which respectively corresponds to binding of the Cy7 monomer and dimer (Figure 5-1B). ${ }^{224,225}$ To assess the binding of Cy7 to various domains within the TWJ pool, we synthesized the isolated S1 stem with an added terminal TTT loop (termed the S1 stem-loop), the S2 stem-loop, and the S3 stemloop (Table 5-1 and Figure 5-3A) and characterized the binding of $\mathrm{Cy} 7$ to each of them. We observed that none of the stem-loops bound to the monomer (Figure 5-3B), and the dimer only bound to the $\mathrm{S} 1 \mathrm{stem}-\mathrm{loop}$ - most likely within its minor groove. ${ }^{225}$ When we incubated $\mathrm{Cy} 7$ with a 1:1:1 mixture of the three isolated stem-loops, only dimer binding was observed (Figure 5-1B). These results clearly indicated that the Cy7 monomer can only bind to the fully-assembled TWJ domain of the aptamer. 
A

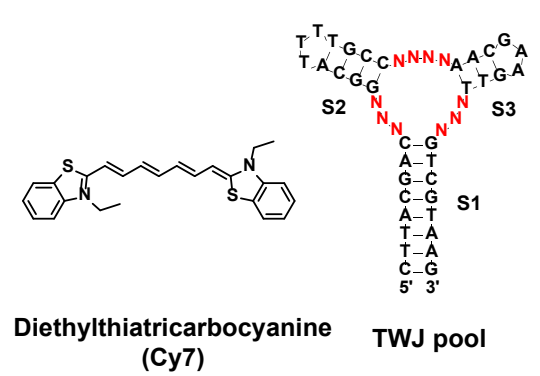

B

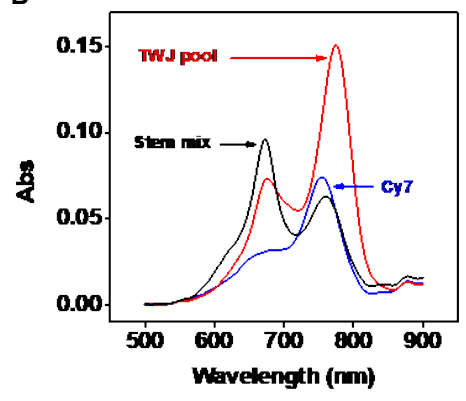

C

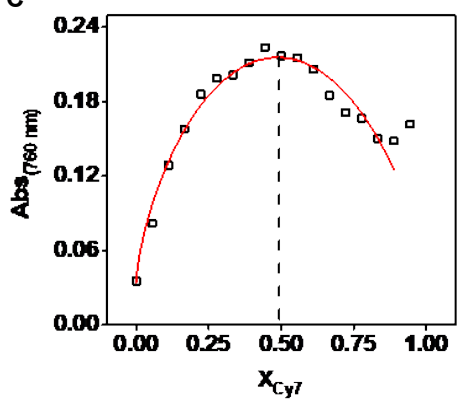

Figure 5-1. Cy7 binding to a TWJ-containing structured DNA pool in a sequence-independent manner. (A) Chemical structure of Cy7 and the sequence of the randomized TWJ pool. (B) When combined with the TWJ pool, the absorbance of Cy7 monomer $(760 \mathrm{~nm})$ and dimer $(670 \mathrm{~nm})$ is enhanced. In contrast, when combined with a 1:1:1 mixture of isolated S1, S2, and S3 stem-loops, only the absorbance of the dimer is enhanced. (C) Binding stoichiometry of Cy7 monomer to the TWJ pool as characterized by a Job plot.
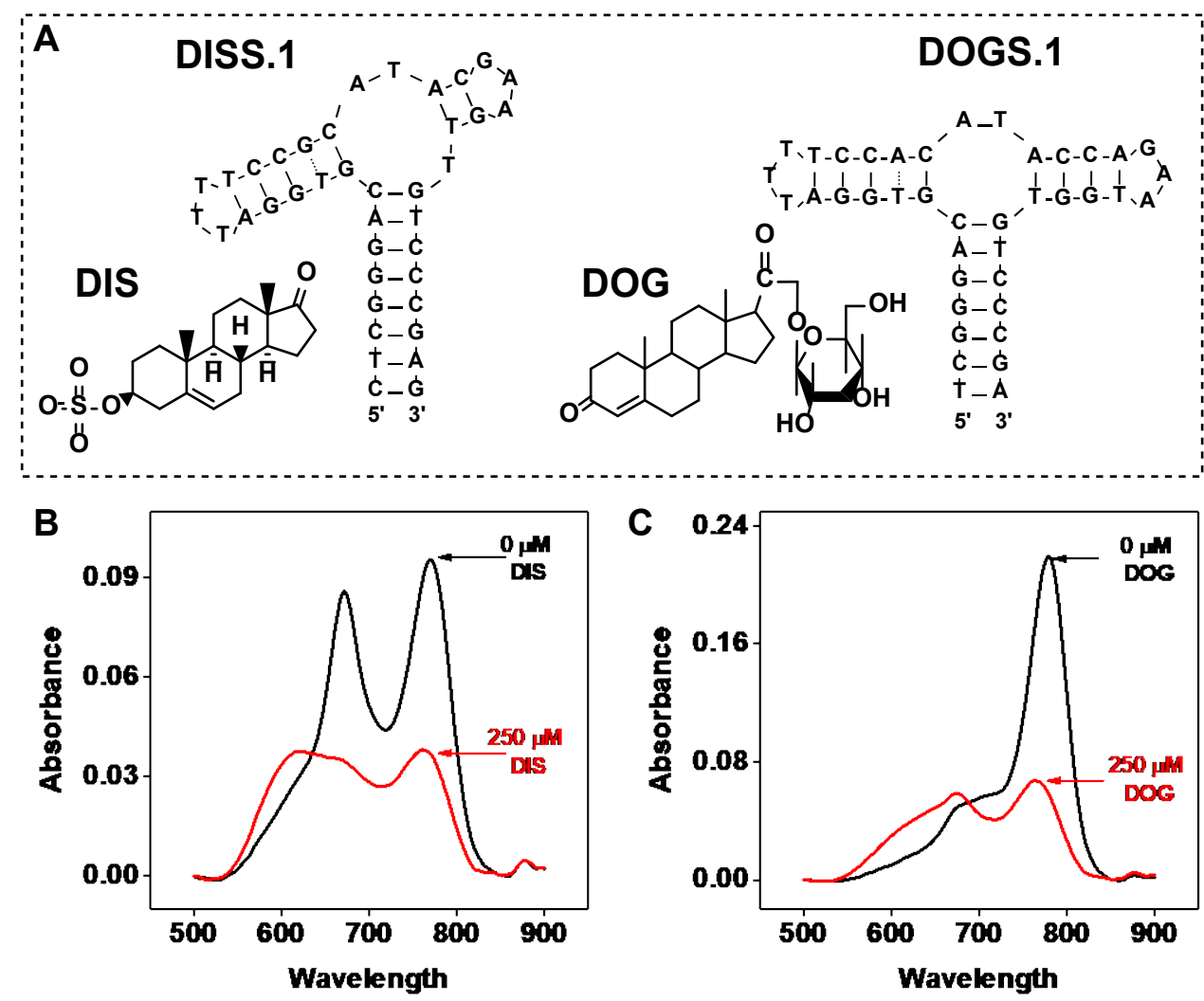

Figure 5-2. Generality of target-induced displacement of Cy7 from aptamers with a TWJstructured binding domain. (A) Structure of DIS and DOG and their respective aptamers, DISS.1 and DOGS.1. Both aptamers contain a TWJ-structured binding domain. Absorbance spectra for a mixture of $3 \mu \mathrm{M}$ Cy7 and $7 \mu \mathrm{M}$ (B) DISS.1 or (C) DOGS.1 in the absence (black line) or presence (red line) of $250 \mu \mathrm{M}$ DIS or DOGS, respectively. The greatly reduced Cy7 absorbance at $760 \mathrm{~nm}$ indicates successful displacement of $\mathrm{Cy} 7$ from the aptamer TWJ domain. 

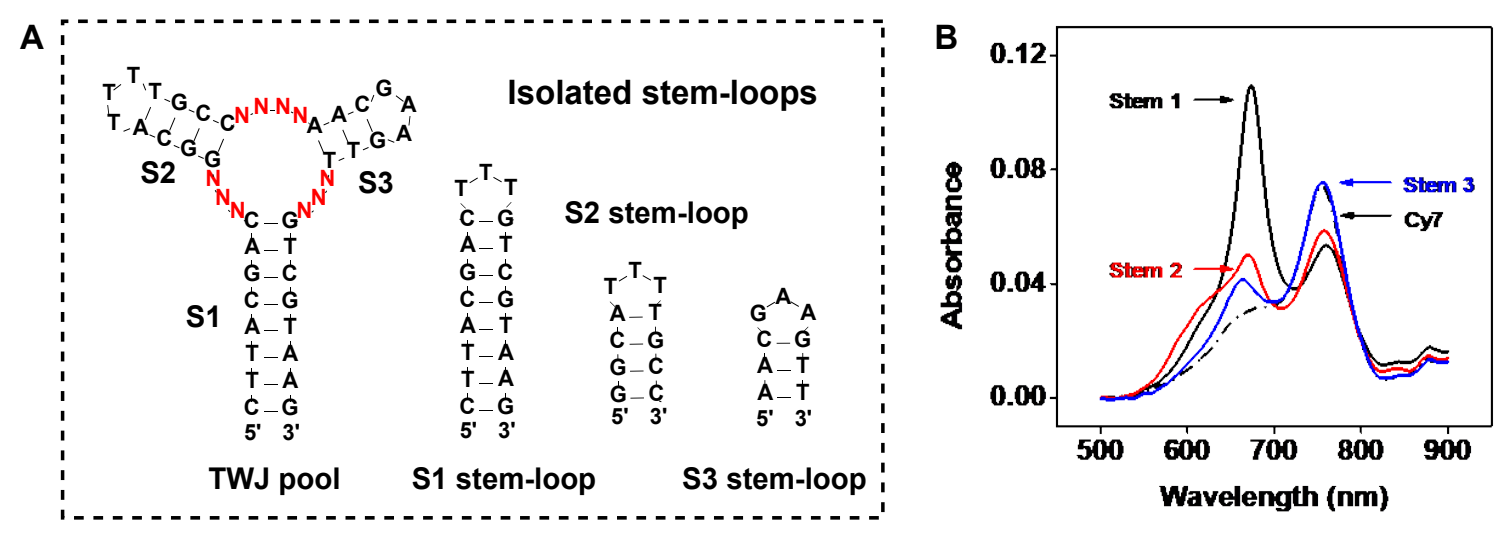

Figure 5-3. Characterization of $\mathrm{Cy} 7$ binding to the isolated stems from the TWJ pool. (A) Sequences of the TWJ pool and isolated S1, S2, and S3 stem-loops. (B) Binding of Cy7 to each isolated stem-loop. When $2 \mu \mathrm{M}$ Cy7 was combined with $10 \mu \mathrm{M}$ S1 stem-loop, only the absorbance of the dimer $(670 \mathrm{~nm})$ increased. Combination with either $10 \mu \mathrm{M}$ S2 stem-loop or S3 stem-loop did not significantly enhance the absorbance of either the monomer or the dimer. The dashed line represents the spectrum of $\mathrm{Cy} 7$ alone.
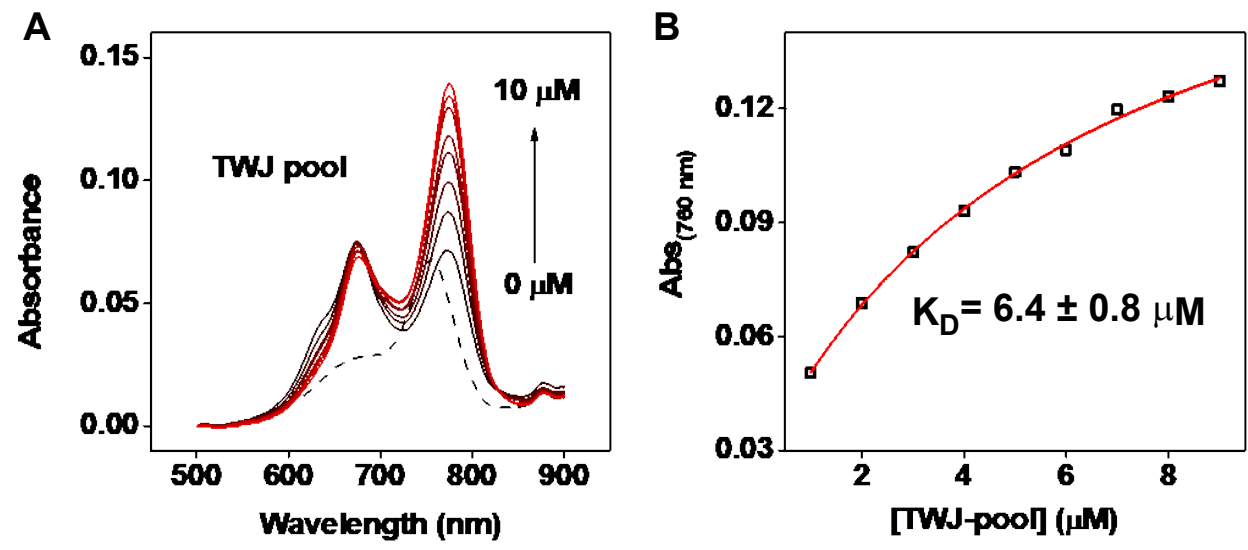

Figure 5-4. Characterization of Cy7 binding to the TWJ pool. (A) Titration of increasing amounts of the TWJ pool $(2-10 \mu \mathrm{M})$ into $2 \mu \mathrm{M}$ Cy 7 resulted in enhanced absorbance of Cy7 at $760 \mathrm{~nm}$, indicating interaction between the TWJ pool and the $\mathrm{Cy} 7$ monomer. The dashed line represents the spectrum of $\mathrm{Cy} 7$ alone. (B) Cy7 binding affinity for the TWJ pool was estimated from absorbance at $760 \mathrm{~nm}$ in the absence and presence of different concentrations of TWJ pool.

We then obtained the binding affinity of $\mathrm{Cy} 7$ monomer for the TWJ pool by titrating different concentrations of the pool into $2 \mu \mathrm{M}$ Cy7. As the concentration of the TWJ pool increased, we observed an increase in absorbance at $760 \mathrm{~nm}$ and a slight decrease in absorbance at $670 \mathrm{~nm}$ (Figure 5-4A). This provided further evidence that the TWJ domain preferentially binds to the $\mathrm{Cy} 7$ monomer. The decreased absorbance of the $\mathrm{Cy} 7$ 
dimer at higher DNA concentrations implies an equilibrium shift from dimer to monomer that favors Cy7 binding to the TWJ pool. Based on the absorbance at $760 \mathrm{~nm}$, we obtained a $\mathrm{K}_{\mathrm{D}}$ of $6.4 \pm 0.8 \mu \mathrm{M}$ (Figure $5-4 \mathrm{~B}$ ). We further characterized the binding stoichiometry of the Cy7 monomer to the TWJ pool using a Job plot ${ }^{226}$. We measured the absorbance at $760 \mathrm{~nm}$ at various $\mathrm{Cy} 7$ :DNA ratios, with a constant total concentration of $5 \mu \mathrm{M}$. The maximum absorbance was observed at a mole fraction $\left(\mathrm{X}_{\mathrm{Cy} 7}\right)$ of 0.5 , indicating that the TWJ pool binds to $\mathrm{Cy} 7$ in a 1:1 ratio. Since the Cy7 monomer exclusively binds to the TWJ domain, each randomized TWJ in the pool should bind to a single Cy7 monomer (Figure 5-1C). These results confirmed our initial hypothesis that the binding of $\mathrm{Cy} 7$ to TWJs is sequence-independent and indicated that it should therefore be feasible to isolate new small-molecule-binding aptamers with intrinsic dyedisplacement functionality from TWJ-structured libraries.

\subsubsection{Library design and isolation process}

We designed a structured library in which we extended both termini of the TWJ pool by 12 nucleotides, creating partial PCR primer binding sites (Figure 5-5 and Table 5-1, library). We have previously demonstrated that changes in the sequence adjacent to the TWJ binding domain greatly affected the affinity of a cocaine-binding aptamer ${ }^{86,102}$. With this in mind, we randomized one base-pair in stems 2 and 3 to further enhance the potential target-binding affinity of our aptamer. The resulting library is designed to primarily form TWJ-structured target-binding domains. Although there is a small possibility of isolating non-TWJ-structured aptamers in the final enriched pool, these sequences can be identified and removed after sequencing. It should be noted that the

randomness of this library ( $4^{14}$ sequences) is considerably lower than many reported 
SELEX libraries. ${ }^{31,32,100}$ However, this should not be of concern, as small-molecule targets can only interact with a limited number of nucleotides within an aptamer's binding domain. In fact, several small-molecule-binding aptamers have been successfully isolated using a TWJ-structured library representing no more than $4^{8}$ different sequences ${ }^{33}$. Increasing the number of random nucleotides in the TWJ binding domain could also lead to the isolation of aptamers with non-TWJ structures, which lack dye-displacement functionality.

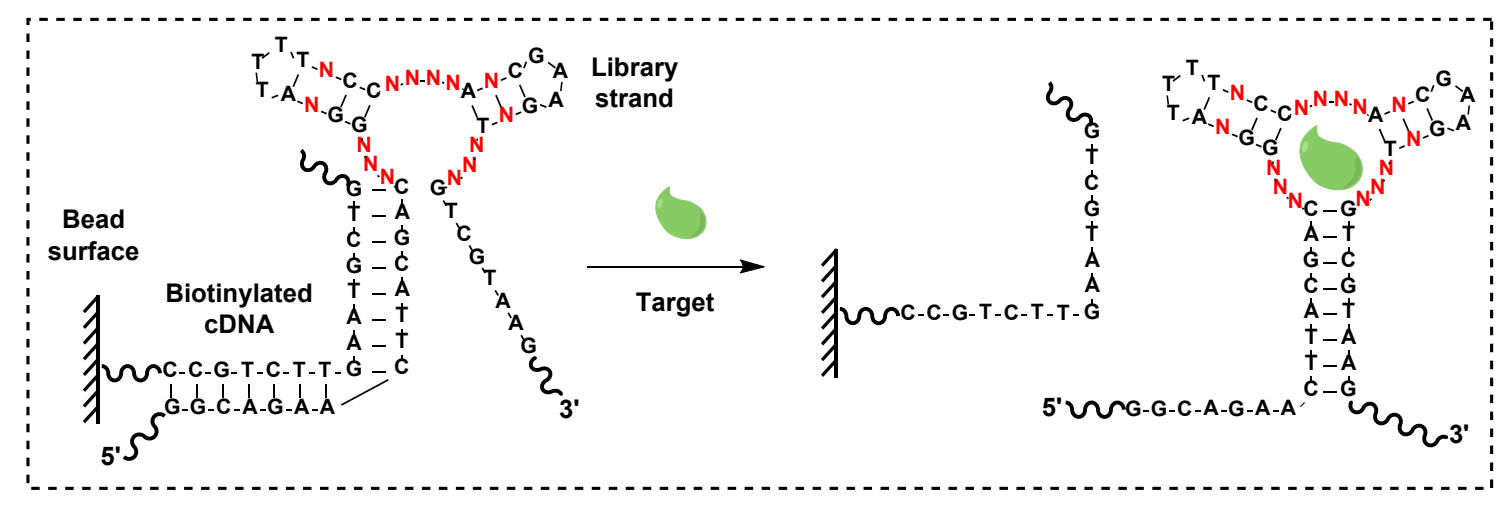

Figure 5-5. Isolation of TWJ-structured aptamers for a Cy7-displacement small-molecule detection assay. A TWJ-containing structured DNA library is immobilized onto a streptavidincoated bead surface using a biotinylated cDNA strand (left). Target-binding strands are eluted due to a conformational change in the aptamer (right).

We then used this TWJ-containing library along with a previously reported libraryimmobilized SELEX strategy ${ }^{33}$ to isolate aptamers for MDPV, an emerging drug of abuse in the synthetic cathinone family. Since there is currently no reliable presumptive test for any synthetic cathinone, the development of such an assay would be highly valuable. We performed SELEX in a low ionic strength buffer $(10 \mathrm{mM}$ Tris-HCl, $20 \mathrm{mM} \mathrm{NaCl}, 0.5$ $\mathrm{mM} \mathrm{MgCl}_{2}, \mathrm{pH}$ 7.4), as an excessive amount of ions can induce non-specific hydrophobic interactions ${ }^{227}$ between the target and the TWJ binding domain, which disfavors the isolation of highly target-specific aptamers. We immobilized our library 
onto streptavidin-coated agarose beads through hybridization to a biotinylated complementary DNA (cDNA) strand (Table 5-1, cDNA-bio) that disrupts the TWJ structure (Figure 5-5A, left). In the presence of MDPV, library molecules that recognized this target underwent a conformational change and refolded into a TWJ structure, thereby detaching themselves from the biotinylated cDNA (Figure 5-5A, right). These aptamers were collected, PCR amplified, and used for the next round of selection. To further improve aptamer specificity, we sequentially performed counter-SELEX at various rounds against three molecules that are structurally similar to MDPV: amphetamine, methamphetamine, and dopamine.
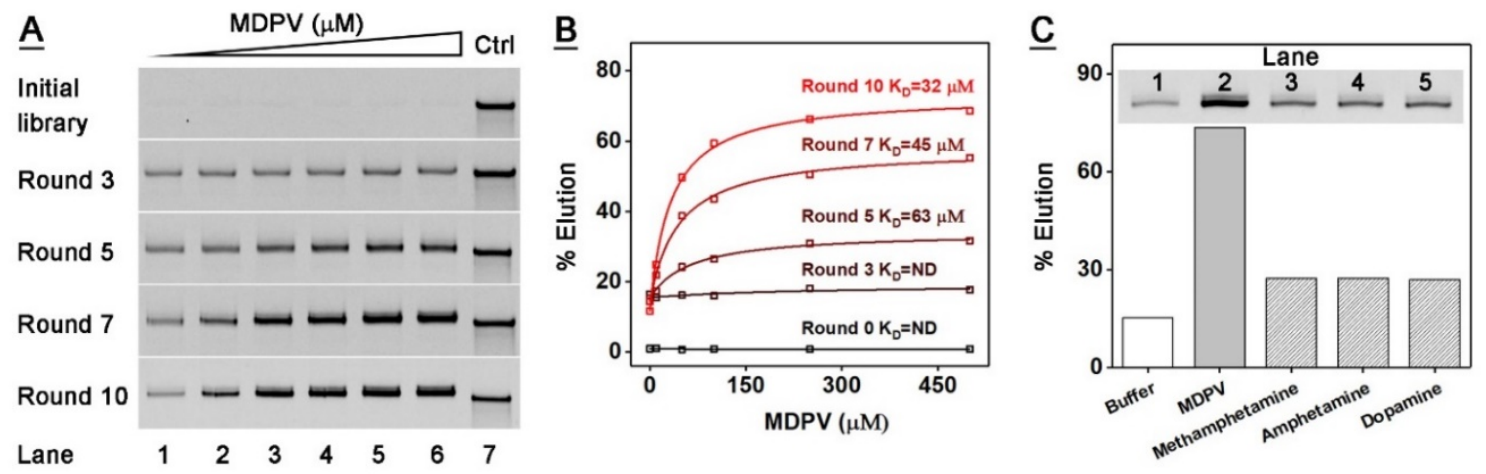

Figure 5-6. Estimation of target affinity and specificity of the enriched pools during SELEX using a target elution assay. (A) Target elution profile of the initial library and enriched pools after selection rounds 3, 5, 7, and 10 was analyzed by PAGE. Lanes 1-6 represent samples of the initial library and enriched pools eluted with $0,10,50,100,250$, and $500 \mu \mathrm{M}$ MDPV. Control samples (lane 7) containing $40 \mathrm{nM}$ synthesized library were used to measure the concentration of eluted strands and percent elution of each sample. (B) The MDPV binding affinity of the library and enriched pools were calculated based on the percent elution of each sample. (C) The target specificity of the enriched pool after 10 rounds of SELEX was also assessed by the target elution assay. Inset shows the enriched pool eluted with binding buffer (lane 1), $500 \mu \mathrm{M}$ MDPV (lane 2), $500 \mu \mathrm{M}$ methamphetamine (lane 3), $500 \mu \mathrm{M}$ amphetamine (lane 4), and $500 \mu \mathrm{M}$ dopamine (lane5).

After each round of SELEX, we estimated the target affinity and specificity of the enriched pool with a target elution assay. After 10 rounds of selection, the enriched pool achieved saturated target affinity and low cross-reactivity to the counter-SELEX targets 
(Figure 5-6). The isolated aptamers were then cloned and sequenced. We observed that all sequences retained the TWJ structure present in the original library. The 10 random nucleotides (Figure 5-7, positions 9-11, 23-26, 36-38) in the TWJ binding domain exhibited a high level of consensus, with 41 out of 50 clones sharing an identical sequence at these sites. The four random nucleotide positions within the stems were more varied (Figure 5-7, positions 14, 20, 28 and 34), but predominantly retained standard Watson-Crick base-pairing. We selected the most highly-represented sequence and truncated the 12-nucleotide partial primer-binding sites at both termini to generate the MDPV-binding aptamer MA (Table 5-1).

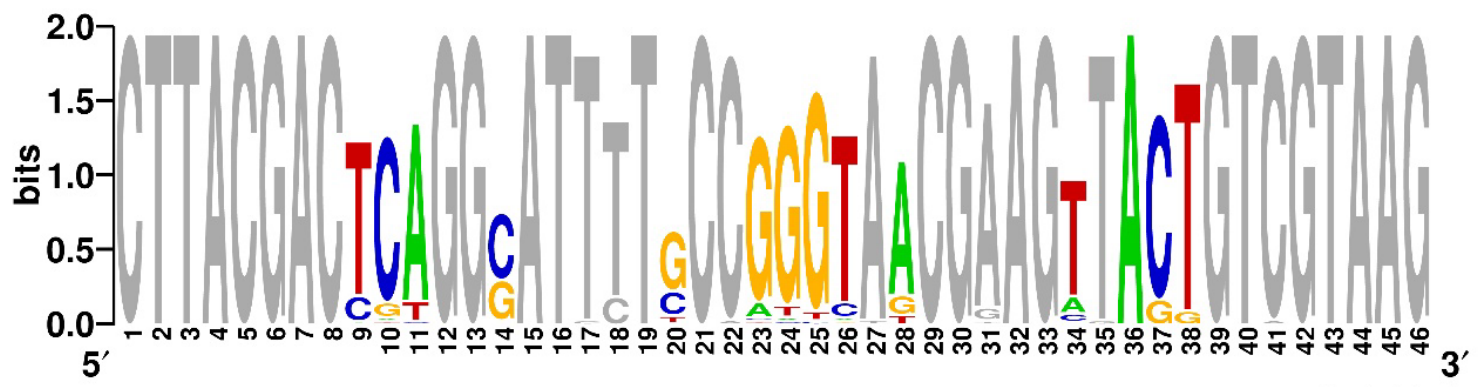

Figure 5-7. Sequence logo for 50 clones isolated after 10 rounds of SELEX, showing the relative frequency of every nucleotide at each position. Nucleotides at pre-determined positions are marked grey, while positions with randomized nucleotides are color-coded to show the distribution of different nucleotides. Higher frequency nucleotides appear in a larger font-size.

\subsubsection{Aptamer characterization and development of the label-free Cy7-}

\section{displacement assay}

We determined the binding affinity of MA using ITC, titrating $1 \mathrm{mM}$ MDPV into a 20 $\mu \mathrm{M}$ solution of the aptamer. We found that MA binds MDPV with a $\mathrm{K}_{\mathrm{D}}$ of $6.1 \pm 0.2 \mu \mathrm{M}$ (Figure 5-8A). Binding is driven by a negative enthalpy change $(\Delta \mathrm{H}=-11.0 \pm 0.2 \mathrm{kcal}$ $\left.\mathrm{mol}^{-1}\right)$ but with a moderate entropy cost $\left(\Delta \mathrm{S}=-13.1 \pm 0.2 \mathrm{cal} \mathrm{mol}^{-1} \mathrm{~K}^{-1}\right)$ due to the fact that MDPV is constrained within the TWJ binding domain. Based on our prior findings, 
we anticipated that $\mathrm{Cy} 7$ would also bind to the TWJ domain of MA but could be displaced by MDPV. This was confirmed by performing an ITC experiment in which we titrated $1 \mathrm{mM}$ MDPV into $20 \mu \mathrm{M}$ Cy7-MA complex (1:1). The presence of Cy7 did not significantly reduce the MDPV-binding affinity of MA $\left(\mathrm{K}_{\mathrm{D}}=7.0 \pm 0.7 \mu \mathrm{M}\right)$ (Figure $5-8 \mathrm{~B})$. However, the binding heat $(\Delta \mathrm{H}=-6.3 \pm 0.2 \mathrm{kcal} / \mathrm{mol})$ was notably lower than in the absence of $\mathrm{Cy} 7\left(\Delta \mathrm{H}=-11.0 \pm 0.2 \mathrm{kcal} \mathrm{mol}^{-1}\right)$, indicating that a certain amount of energy was required for target-induced displacement of the dye. Additionally, the higher binding entropy obtained with $\mathrm{Cy} 7\left(\Delta \mathrm{S}=0.5 \pm 0.7 \mathrm{cal} \mathrm{mol}^{-1} \mathrm{~K}^{-1}\right)$ suggested positive entropy associated with the MDPV-mediated release of the dye from the aptamer. These results indicate that both $\mathrm{Cy} 7$ and MDPV can bind to the TWJ-structured binding domain, and that MDPV can efficiently displace Cy7.
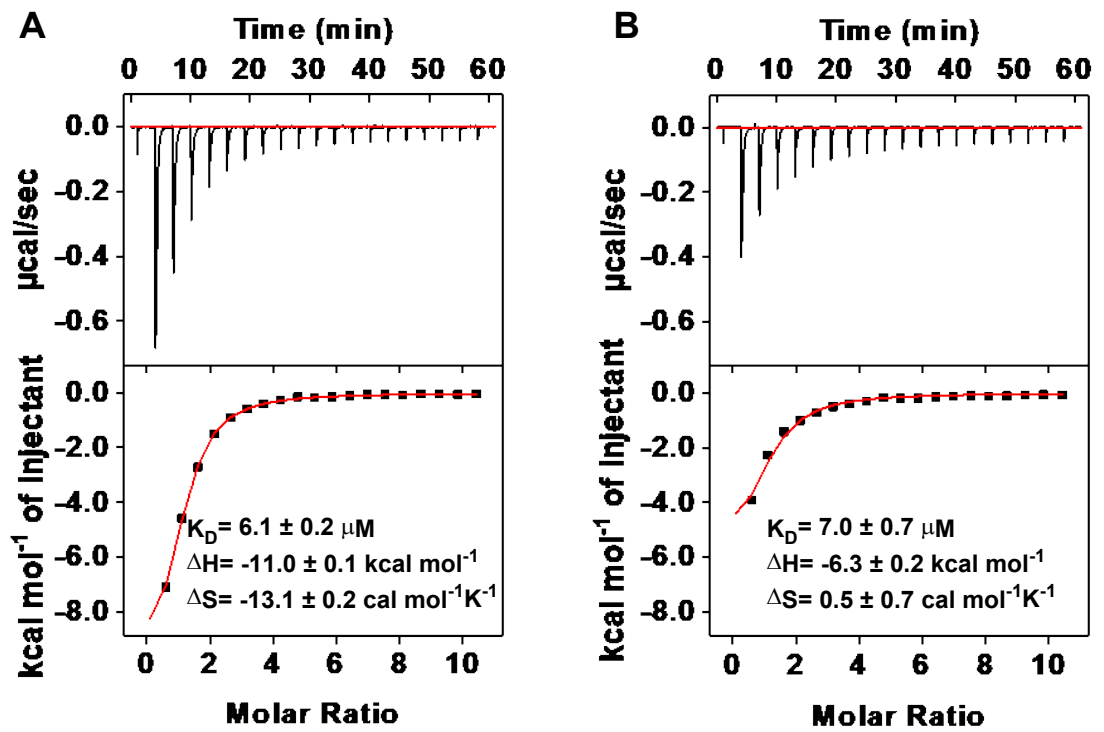

Figure 5-8. Binding affinity of MDPV to MA and MA-Cy7 complex. ITC characterization of MA binding to MDPV in the (A) absence or (B) presence of Cy7 are shown. The top panels display raw data, showing the heat generated from each titration of MDPV. The bottom panels show the integrated heat of each titration after correcting for dilution heat of the titrant.

We then employed MA in a label-free colorimetric assay for the detection of MDPV, where target-induced displacement of $\mathrm{Cy} 7$ from the aptamer TWJ binding domain 
produces a change in dye absorbance (Figure 5-9A). Specifically, we added different concentrations of MDPV $(0.1-640 \mu \mathrm{M})$ to a solution containing Cy7-MA complexes, and then immediately began recording the absorption spectra. Within seconds, we observed a progressive reduction of absorbance at $760 \mathrm{~nm}$ and enhancement of absorbance at 670 $\mathrm{nm}$ (Figure 5-9B). Using the absorbance ratio at $670 / 760 \mathrm{~nm}$ as an indicator, we obtained a linear range from $0-40 \mu \mathrm{M}$, with a low detection limit of $300 \mathrm{nM}$ (Figure 5-9C).
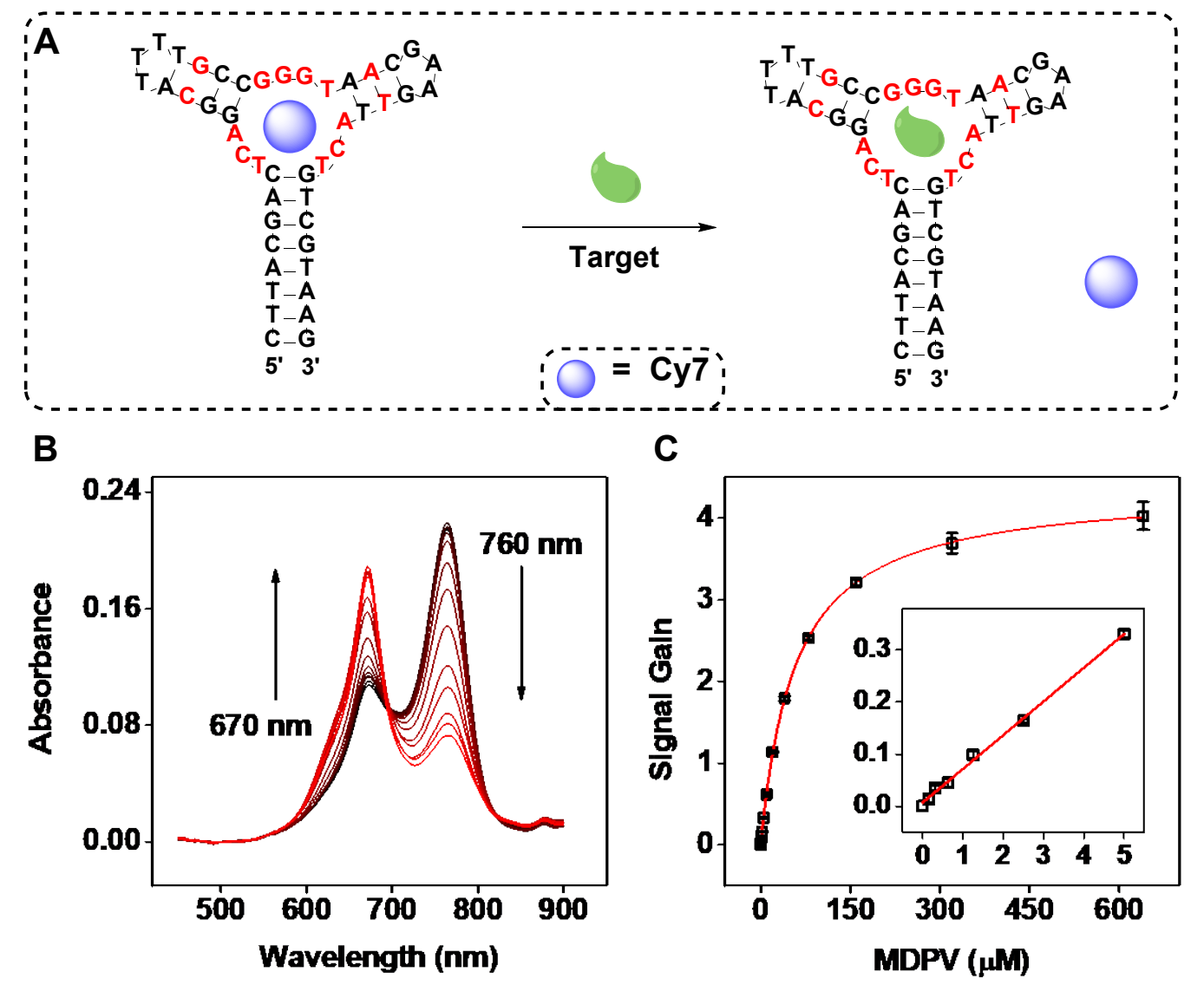

Figure 5-9. Detection of MDPV with a label-free Cy7-displacement colorimetric assay. (A) Cy7 binds within the TWJ domain of MA (left). MDPV displaces Cy7 from the binding domain (right) to generate a change in the absorbance spectra of Cy7. (B) UV-vis spectra of $3 \mu \mathrm{M}$ Cy7 premixed with $7 \mu \mathrm{M}$ MA in the presence of varying concentrations of $\operatorname{MDPV}(0,0.16,0.31,0.63$, $1.25,2.5,5,10,20,40,80,160,320,640 \mu \mathrm{M}$ ). The increasing concentrations (shown as black $\rightarrow$ red curves) resulted in decreased absorbance at $760 \mathrm{~nm}$ and increased absorbance at 670 $\mathrm{nm}$. (C) A calibration curve based on the absorbance ratio at $670 / 760 \mathrm{~nm}$. Inset shows assay performance at a low concentration range. Error bars show standard deviation from three measurements at each concentration. 
A

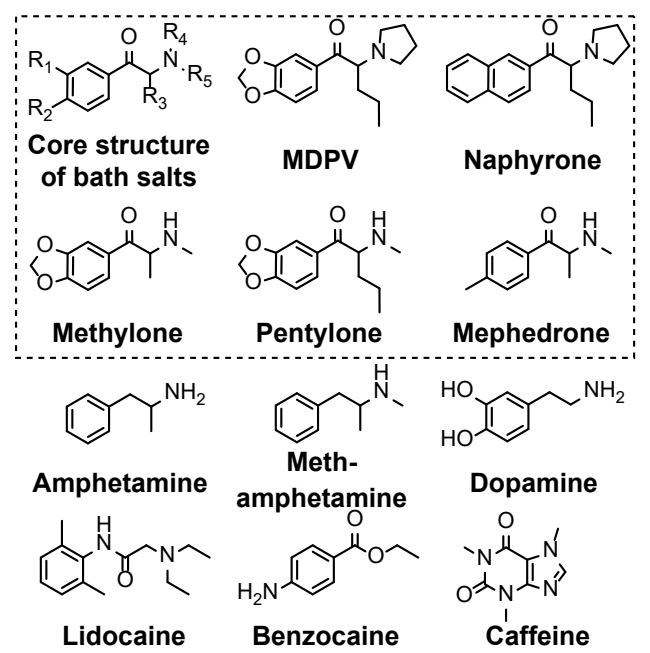

B

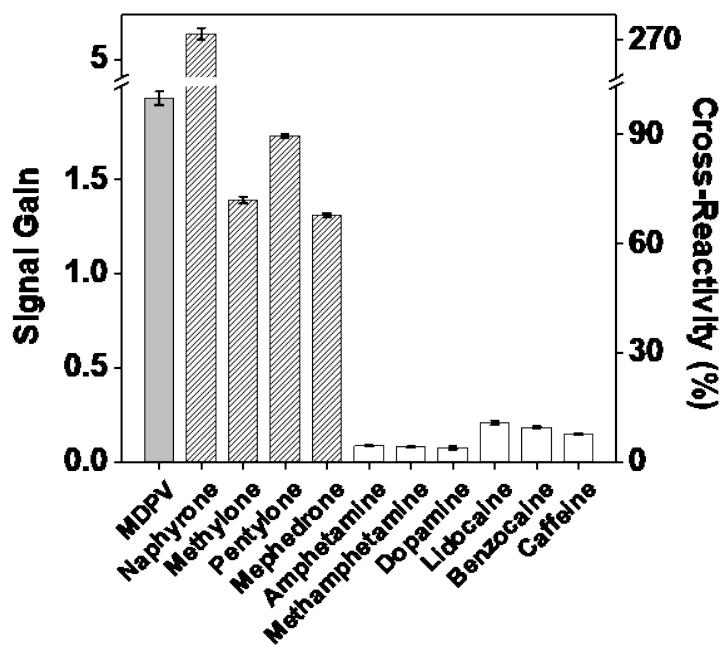

Figure 5-10. High cross-reactivity of MA to synthetic cathinone drugs and high specificity against other structurally-similar or -dissimilar interfering agents. (A) Chemical core structure and structures of synthetic cathinones. (B) Signal gain measurements from the Cy7-displacement assay with $50 \mu \mathrm{M}$ MDPV, other synthetic cathinones, or interfering agents (structures shown in panel C). Error bars show standard deviations from three measurements of each compound.

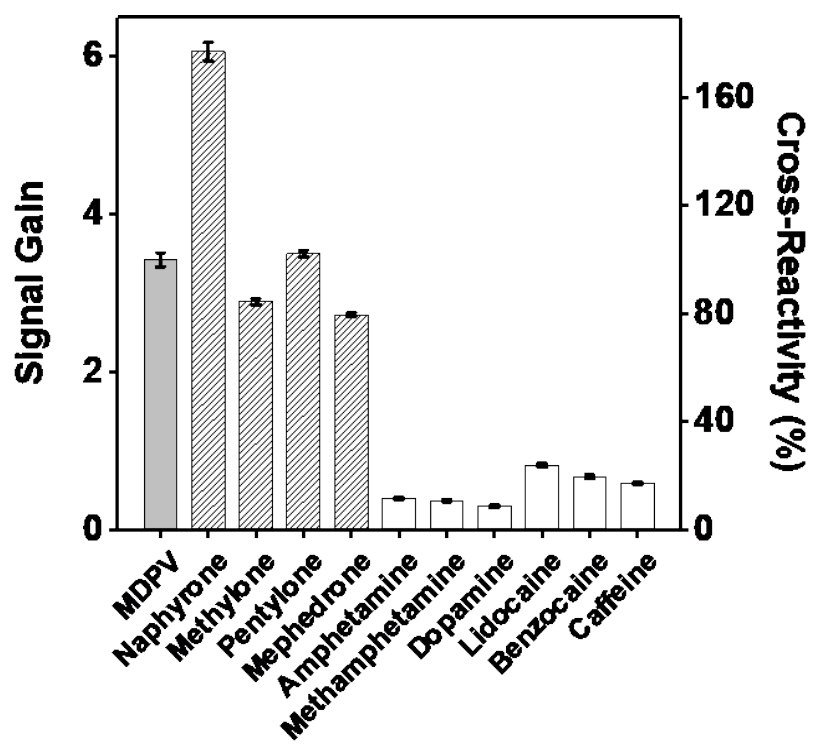

Figure 5-11. Cross-reactivity and specificity of our Cy7-displacement assay with $250 \mu \mathrm{M}$ MDPV, other synthetic cathinones, or structurally-similar non-cathinone compounds and common cutting agents. Signal gain was calculated by $\left(\mathrm{R}-\mathrm{R}_{0}\right) / \mathrm{R}_{0}$, where $\mathrm{R}$ and $\mathrm{R}_{0}$ are the absorbance ratio at $670 / 760 \mathrm{~nm}$ with and without target/interfering agent, respectively. Error bars show standard deviation from three measurements of each compound tested. 
Notably, MA was highly cross-reactive to other synthetic cathinones (methylone, pentylone, naphyrone and mephedrone) that share the same beta-keto phenethylamine core structure as MDPV but with different side-chains (Figure 5-10A). We tested MA cross-reactivity to these four synthetic cathinones at concentrations of $50 \mu \mathrm{M}$ and $250 \mu \mathrm{M}$ in the Cy7-displacement assay. We observed cross-reactivity greater than $65 \%$ and $80 \%$ for all four molecules at both concentrations (Figure 5-10B and Figure 5-11). This suggests that MA specifically recognizes the core structure of synthetic cathinones rather than their side chain substituents. Interestingly, naphyrone showed exceptionally high cross-reactivity compared to other synthetic cathinones, possibly due to the hydrophobicity of its naphthalene moiety. The high cross-reactivity of MA is desirable for onsite detection of the synthetic cathinone family, a class of designer drugs for which new derivatives are continually being developed. In contrast, MA was not responsive to non-synthetic cathinone interfering agents. No specific signal was observed from any of our three counter-SELEX targets (Figure 5-10C) in our Cy7-displacement assay at concentrations of $50 \mu \mathrm{M}$ (Figure 5-10B) or $250 \mu \mathrm{M}$ (Figure 5-11), despite their structural similarity to MDPV. We further tested the specificity of the assay against lidocaine, benzocaine, and caffeine, which are cutting agents commonly found in seized substances (Figure 5-10C), and likewise observed little cross-reactivity at either concentration (Figure 5-10B and Figure 5-11). It should be noted that the counter-SELEX targets and cutting agents either do not contain all the moieties present in MDPV (a phenyl ring, a ketone, and an amine group), or have all of these moieties but arranged in different positions. These results indicate that MA recognizes targets based on multiple specific interactions in a certain arrangement. We found that MA was somewhat cross-reactive to 
the structurally dissimilar compounds cocaine, DIS, and DOG at a concentration of 50 $\mu \mathrm{M}$, with cross-reactivities of $50 \%, 18 \%$, and $40 \%$ respectively relative to MDPV (Figure 5-12) in the Cy7-displacement assay. We believe this cross-reactivity occurs because counter-SELEX was not performed against these particular compounds, but it should be feasible to eliminate this unintended cross-reactivity with well-designed counter-SELEX procedures against additional structurally dissimilar compounds.

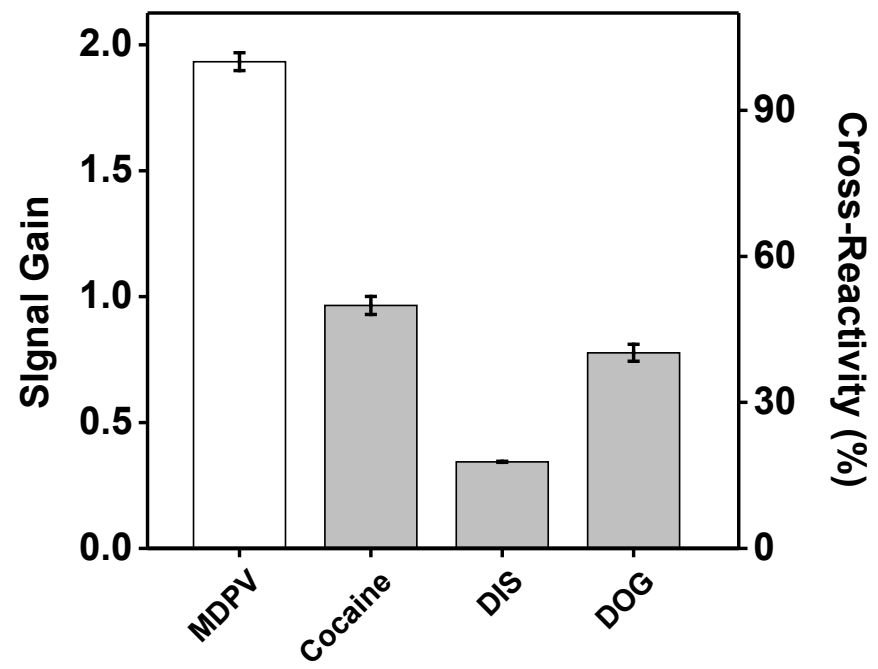

Figure 5-12. Cross-reactivity of our Cy7-displacement assay with $50 \mu \mathrm{M}$ MDPV, cocaine, DIS and DOG. Error bars show standard deviation from three measurements of each compound.

\subsubsection{Comparison of $\mathrm{Cy} 7$-displacement and strand-displacement assays}

Finally, we compared the sensitivity of our Cy7-displacement colorimetric assay with a strand-displacement fluorescence assay (Figure 5-13) based on the same MDPV-binding aptamer. We generated a fluorophore-modified version of MA (Table 5-1, MA-F) and a quencher-modified complementary strand (Table 5-1, cDNA-Q). In the absence of MDPV, MA-F forms a 15-bp duplex with cDNA-Q that situates the fluorophore in close proximity to the quencher (Figure 5-13A). The fluorescence of $50 \mathrm{nM}$ MA-F was almost completely quenched in the presence of $50 \mathrm{nM}$ cDNA-Q (Figure 5-14A). We 
subsequently titrated different concentrations of cDNA-Q into $50 \mathrm{nM}$ MA-F and obtained a $\mathrm{K}_{\mathrm{D}}$ of $0.5 \pm 0.2 \mathrm{nM}$ (Figure 5-14A, inset). In the presence of MDPV, the target induced a conformational change in MA-F, forming a TWJ structure that resulted in dissociation of cDNA-Q, generating a measurable fluorescence signal (Figure 5-13B). We observed less than $50 \%$ fluorescence recovery upon adding various concentrations of MDPV up to $640 \mu \mathrm{M}$ (Figure 5-14B). We obtained a linear range of $0-20 \mu \mathrm{M}$, with a detection limit of $2.5 \mu \mathrm{M}$ (Figure 5-14C), eight-fold poorer than the Cy7-displacement assay. This is primarily because cDNA-Q has a much higher affinity for the aptamer than $\mathrm{Cy} 7$, which makes target-induced displacement of the complementary strand less energetically favorable. To further illustrate this point, we synthesized unmodified versions of MA-F and cDNA-Q (Table 5-1, MA-L and cDNA) and performed ITC experiments in which we titrated $1 \mathrm{mM}$ MDPV into $20 \mu \mathrm{M}$ of MA-L either with or without $20 \mu \mathrm{M}$ cDNA. We found that MA-L has an affinity for MDPV almost identical to that of MA (Figure 5-15A, $\left.\mathrm{K}_{\mathrm{D}}=6.2 \mu \mathrm{M}\right)$ in the absence of cDNA. However, in the presence of $20 \mu \mathrm{M}$ cDNA, no MDPV binding was observed (Figure 5-15B), confirming that MDPV could not efficiently displace the cDNA from the aptamer. It should be noted that stranddisplacement efficiency was lower in the ITC experiment due to the higher concentration of aptamer-cDNA complex employed $(20 \mu \mathrm{M})$, which greatly reduces the level of dissociation of this complex. As a control, we titrated $1 \mathrm{mM}$ MDPV into $20 \mu \mathrm{M}$ Cy7MA-L (1:1), and found that the presence of Cy7 does not attenuate MDPV binding by MA-L (Figure $5-15 \mathrm{C}, \mathrm{K}_{\mathrm{D}}=7.0 \mu \mathrm{M}$ ). These results clearly demonstrate that the Cy7displacement assay is more sensitive than an equivalent strand-displacement assay for the detection of small-molecule targets. 

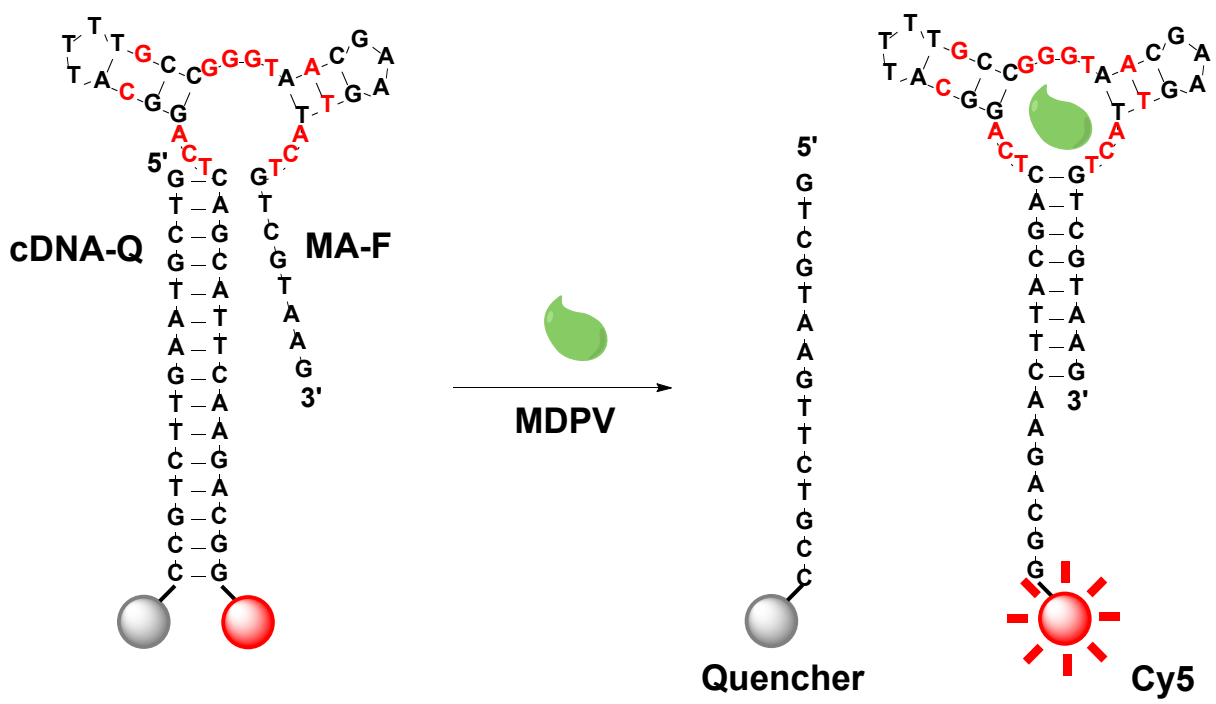

Figure 5-13. Working principle of a conventional strand-displacement fluorescence assay. (A) In the absence of MDPV, MA-F forms a 15-bp duplex with cDNA-Q, which situates the fluorophore in close proximity to the quencher, thus quenching fluorescence. (B) MDPV induces a targetinduced conformational change in MA-F, forming a TWJ structure that results in dissociation of cDNA-Q, generating a measurable fluorescence signal.
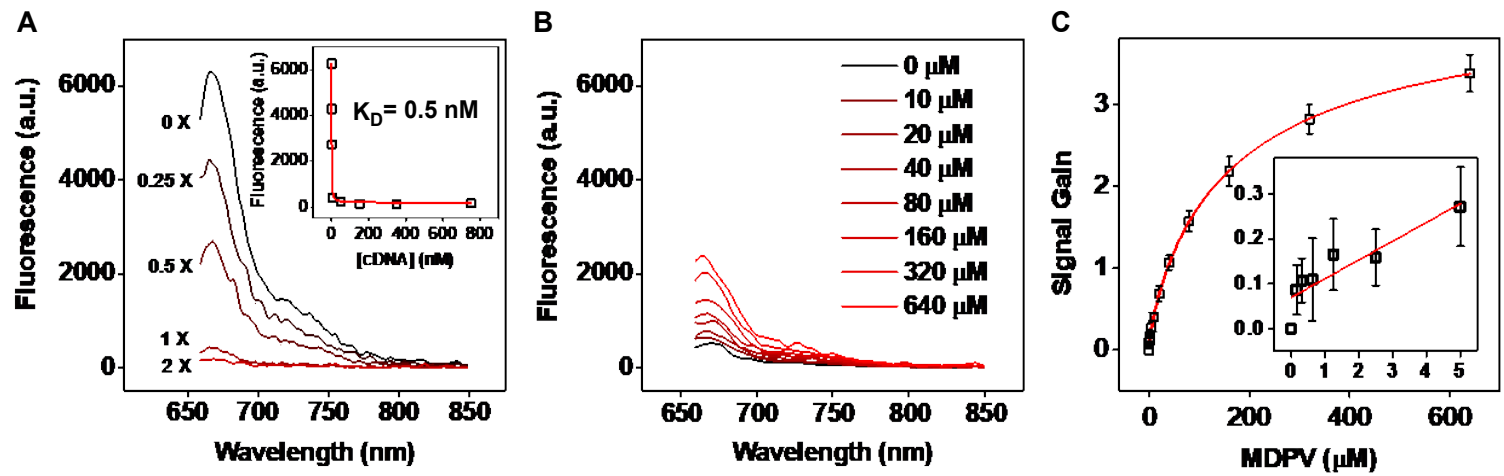

Figure 5-14. Strand-displacement fluorescence assay based on the MA aptamer. (A) Fluorescence spectra obtained from titration of cDNA-Q into a solution of MA-F at different molar ratios $(0-2 \times)$. Inset shows binding affinity of cDNA-Q to MA-F as estimated from the fluorescence intensity at $668 \mathrm{~nm}$ in the absence and presence of different concentrations of cDNA-Q. (B) Fluorescence spectra obtained from titration of different concentrations of MDPV into $50 \mathrm{nM}$ MA-F and $50 \mathrm{nM}$ cDNA-Q. (C) Calibration curves for the strand-displacement fluorescence assay. Inset shows assay performance at a low concentration range. Error bars show standard deviation from three measurements at each concentration. 

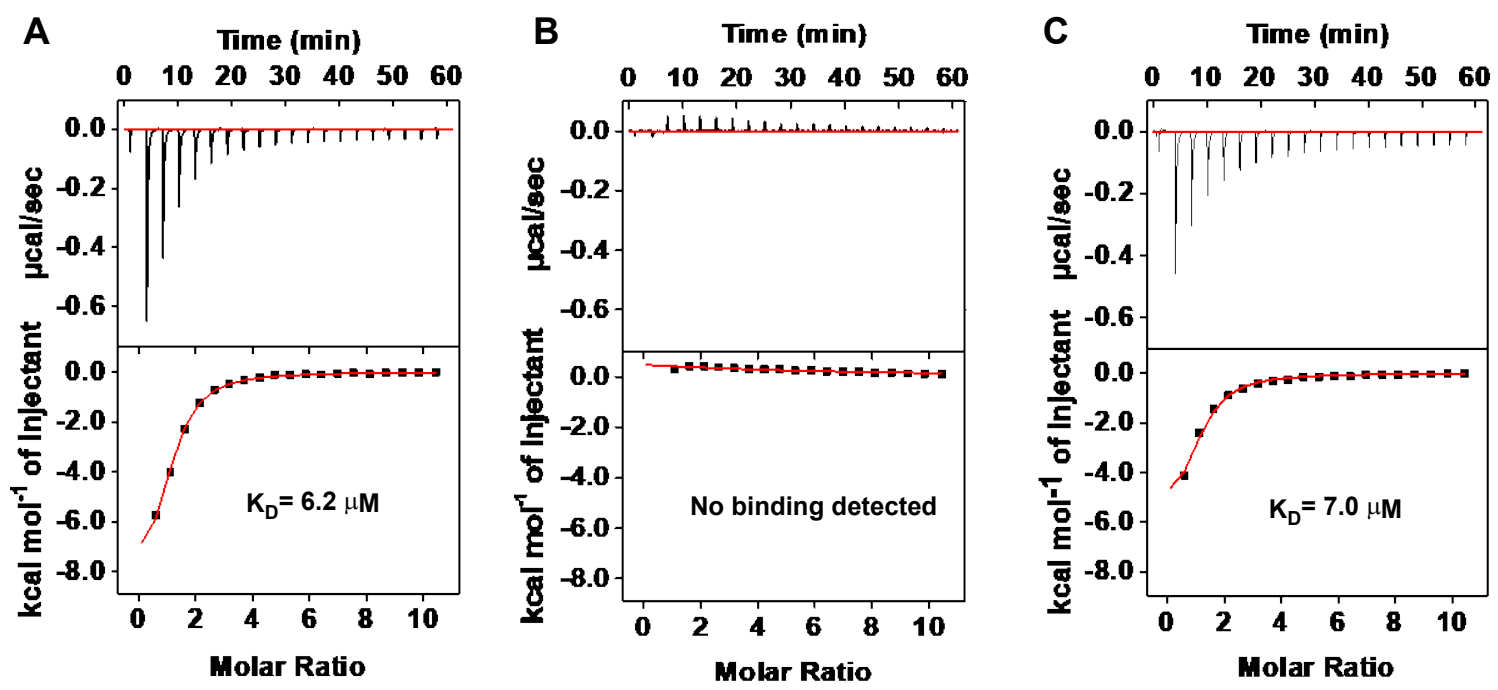

Figure 5-15. Affinity of MDPV to MA-L, cDNA-MA-L complex, and Cy7-MA-L complex. MDPV binding affinity to (A) MA-L alone, (B) cDNA-MA-L complex (1:1), and (C) Cy7-MA-L complex $(1: 1)$ are characterized by ITC. The top panels display raw data showing the heat generated from each titration of MDPV. The bottom panels show the integrated heat of each titration after correcting for dilution heat of the titrant.

\subsection{Conclusions}

In this report, we describe a general approach for the isolation of small-moleculebinding aptamers with intrinsic dye-displacement functionality that can be directly implemented in a label-free colorimetric assay. We first determined that Cy7 can bind to a randomized TWJ-structured DNA library pool in a sequence-independent manner. We then used a TWJ-structured library to isolate a DNA aptamer against MDPV, an emerging designer drug, and directly employed the isolated aptamer in a Cy7displacement colorimetric assay without any further engineering. The assay showed high target sensitivity, detecting MDPV at concentrations as low as $300 \mathrm{nM}, 20$-fold lower than the $\mathrm{K}_{\mathrm{D}}$ of the aptamer, within seconds at room temperature. Our aptamer showed high specificity in terms of discriminating against structurally-similar non-synthetic cathinone compounds such as amphetamine, methamphetamine, and dopamine as well as common cutting agents such as lidocaine, benzocaine, and caffeine. At the same time, the 
isolated aptamer was highly cross-reactive to other synthetic cathinones such as naphyrone, methylone, pentylone, and mephedrone, indicating that it could offer a broadly applicable yet specific assay for this particular class of designer drugs, based on recognition of shared core structural features.

Our determination that Cy7 can generally bind our DNA TWJs in a sequenceindependent manner means that such dye-displacement assays can be generalized for the detection of other small-molecule targets using existing or future isolated TWJ-structured aptamers. Since we have previously demonstrated that the fluorescent dye ATMND can bind to the TWJ domain of a cocaine-binding aptamer, ${ }^{102}$ we anticipate that other hydrophobic small-molecule dyes may also serve as signal reporters in such dyedisplacement assays. Our assay is simple, rapid, sensitive, and cost efficient, as it can be performed by simply mixing the sample, Cy7, and unmodified aptamer. The Cy7 absorbance read-out can be quantified in seconds by a microplate-reader or portable photometer, allowing for high-throughput or on-site detection, respectively. Importantly, the absorbance of Cy7 at $670 \mathrm{~nm}$ and $760 \mathrm{~nm}$ exhibits minimal overlap with other small molecules ${ }^{228}$ or sample matrices, ${ }^{229}$ potentially allowing for interference-free detection in complex specimens. Finally, our assay is eight-fold more sensitive than an equivalent strand-displacement fluorescence assay, because $\mathrm{Cy} 7$ is more efficiently displaced by small-molecule targets than a hybridized cDNA strand. Therefore, we believe that our SELEX strategy using our TWJ-structured library could offer a broad foundation for the development of sensitive and simple Cy7-displacement colorimetric assays for the detection of a wide variety of small-molecule targets. 
CHAPTER 6: In Vitro Isolation of Class-Specific Oligonucleotide-Based Small-

\author{
Molecule Receptors
}

\title{
6.1. Introduction
}

It is highly advantageous to be able to sensitively detect multiple different members of a particular molecular family or class in many analytical contexts-for example, detecting illicit drugs and their metabolites for forensic investigations, antibiotics for food safety, or pesticides for environmental monitoring. ${ }^{67-69}$ Cross-reactive assays that can broadly detect small molecules based on a shared molecular framework offer a more efficient and cost-effective solution to this problem than the tandem use of multiple highly specific assays that each detects an individual analyte. Antibody-based immunoassays have dominated the field of small-molecule detection, ${ }^{230}$ and while assays have been developed for a wide variety of individual targets, the development of classspecific immunoassays has proven difficult. ${ }^{231,232}$ This is in part because the process of antibody generation, which is performed in vivo, provides no control over the targetbinding affinity and spectrum of the resulting antibody. Nucleic-acid-based affinity reagents known as aptamers hold much promise in circumventing many of the shortcomings associated with antibodies. ${ }^{76}$ Aptamers are isolated through SELEX to bind targets of interest with high affinity and specificity. Unlike antibodies, aptamers can be isolated relatively quickly and chemically synthesized in an inexpensive manner with no batch-to-batch variation. Moreover, aptamers are thermostable and have shelf lives of a few years at room temperature. ${ }^{15}$

Theoretically, since SELEX is an in vitro process, the selection strategy and conditions can be precisely controlled to isolate class-specific aptamers that can broadly 
bind to small molecules sharing the same core structure. However, little work has been done to demonstrate the capability of SELEX to achieve such a goal. Aptamers isolated for a given small-molecule target often have innate cross-reactivity to analogs of that molecule, but the target-binding spectra of these aptamers are often either insufficient and/or unpredictable. For example, one heavily studied cocaine-binding aptamer ${ }^{21}$ can also bind to metabolites such as norcocaine and cocaethylene, but does not respond to the major metabolite benzoylecgonine, which only differs from cocaine by a single methyl group. ${ }^{88,233}$ Toggle-SELEX was developed as a solution to isolate cross-reactive aptamers. ${ }^{26}$ In this strategy, a library pool is challenged with two different targets sharing the same core structure, which are alternated every round to select for an aptamer that can cross-react to both targets - and ideally, target analogs sharing the same core structure. This method has led to the isolation of cross-reactive aptamers for a few structurallyrelated small molecules. ${ }^{36,39,73}$ However, these aptamers typically exhibit only limited cross-reactivity, ${ }^{39,73}$ and the overall success rate of such approaches has been low. ${ }^{36}$ For example, Derbyshire et al. successfully isolated an aptamer that can bind to eight aminoglycoside antibiotics using Toggle-SELEX. Out of four independent selections with four different target pairs, and only one yielded the final aptamer. ${ }^{36}$ The limitations of Toggle-SELEX could be attributed to two reasons. First, in previously reported approaches, only a fraction of possible substituent positions was varied between the two targets, yielding aptamers with narrow target-binding spectra. Second, since only a small portion of the aptamers in the initial pool are cross-reactive compared to those that bind to at least one target, those cross-reactive aptamers could be lost during Toggle-SELEX. 
To overcome these problems, we have developed a new 'parallel-and-serial' selection strategy for SELEX to isolate class-specific aptamers for small molecule families. Our strategy has three steps that are crucial for its success. First, a set of structurally-related targets are selected to define the core structure that is to be recognized by the isolated aptamer. The use of these targets creates selection pressure for aptamers that recognize generic molecular features common to all targets while remaining insensitive to peripheral substituents. Accordingly, it is important to choose as many targets as needed to represent variations at all desired substituent sites while preserving the core molecular framework of the target family. Next, these various targets are employed in a parallel selection process, in which multiple aptamer pools are enriched using each individual target. As a result, cross-reactive aptamers recognizing the shared core structure are enriched in all of the resulting pools after a few rounds of selection. When all these parallel pools are combined, the population of such aptamers should be relatively high. Finally, this combined pool is subjected to serial selection with each target sequentially, a process that ultimately retains only those aptamers that bind to the core structure shared by these targets. Importantly, this selection strategy is supplemented with a well-designed counter-SELEX procedure ${ }^{25}$ to further define the targeted core structure and to prevent the aptamer from binding to structurally-similar non-target molecules.

As a demonstration of this strategy, we isolated a class-specific aptamer for synthetic cathinones, a large family of dangerous designer drugs ${ }^{173}$ that are associated with many severe psychological and physiological health consequences. ${ }^{174,175}$ The isolated aptamer demonstrated low nanomolar binding affinity to 12 synthetic cathinones while having no response to 11 structurally-similar non-cathinone molecules. Analysis of the aptamer 
isolation process via high throughput sequencing revealed that cross-reactive sequences were enriched during parallel selection, and that exponential enrichment of such aptamers occurred during serial selection. The target-binding spectrum of the aptamer was further evaluated via a dye-displacement sensing platform. Impressively, the aptamer enabled instantaneous colorimetric detection of several synthetic cathinones at nanomolar concentrations in biological samples, rivaling the performance of any currently-available immunoassays which can only detect a few members of this family. To our knowledge, this is the first demonstration of using a rationally-designed strategy to isolate highaffinity aptamers that are truly class-specific, having both broad target-binding spectrum as well as excellent specificity. Such success shows that the customizable nature of SELEX makes it well-suited for sourcing receptors with specific, yet broad molecular recognition capabilities, and that the approach described herein can be generally employed to directly isolate class-specific aptamers for any small-molecule family.

\subsection{Experimental section}

\subsubsection{Materials}

All DNA oligonucleotides were purchased from Integrated DNA Technologies (HPLC-purified) and dissolved in PCR water. The names and sequences of the DNA oligonucleotides are listed in

Table 6-1. The concentrations of dissolved DNA were measured using a NanoDrop 2000 spectrophotometer (Thermo Fisher Scientific). Synthetic cathinone standands (all hydrochloride salts and racemic unless specified), including 3,4-methylenedioxy- $\alpha$ pyrrolidinobutiophenone (MDPBP), 3,4-methylenedioxypyrovalerone (MDPV), 3- 
fluoromethcathinone (3-FMC), 4-fluoromethcathinone (4-FMC), 4-methylapyrrolidinobutiophenone (MePBP), 4'-methyl- $\alpha$-pyrrolidinohexiophenone (MPHP), 4methylmethcathinone (4-MMC), alpha-pyrrolidinopentiophenone ( $\alpha$-PVP), butylone, cathinone, ethylone, methcathinone, methedrone, methylone, naphyrone, pentylone, pyrovalerone, and pure enantiomers (-)-MDPV and (+)-MDPV were purchased from Cayman Chemicals. Acetaminophen, $( \pm)$-amphetamine hemisulfate, benzocaine, caffeine, cocaine $\mathrm{HCl}$, diethylthiatricarbocyanine iodide (Cy7), (-)-ephedrine $\mathrm{HCl}$, lidocaine $\mathrm{HCl}$, $(+)$-methamphetamine $\mathrm{HCl}$, procaine $\mathrm{HCl}$, promazine $\mathrm{HCl},(+)$-pseudoephedrine $\mathrm{HCl}$, sucrose and all other chemicals were purchased from Sigma-Aldrich unless otherwise noted. Tween 20, formamide, SYBR Gold, streptavidin-coated agarose resin (capacity: 1-3 mg biotinylated BSA $/ \mathrm{ml}$ resin), One Shot Chemically Competent E. coli, TOPO TA cloning kit, PureLink Quick Plasmid Miniprep Kit, and ExoSAP-IT Express PCR Purification Kit were purchased from ThermoFisher Scientific. $500 \mu \mathrm{L}$ micro-gravity columns were purchased from Bio-Rad. GoTaq Hot Start Colorless Master Mix was purchased from Promega. $3 \mathrm{kDa}$ cut-off spin filters were purchased from Millipore.

Table 6-1. Sequences of DNA oligonucleotides employed in Chapter 6.

\begin{tabular}{ll}
\hline Sequence ID & Sequence $\left(5^{\prime}-3^{\prime}\right)$ \\
\hline DNA Library & CGAGCATAGGCAGAACTTACGAC(N30) \\
GTCGTAAGAGCGAGTCATTC \\
FPN-bio & TTTTTGTCGTAAGTTCTGCCATTTT/Bio/ \\
RP-bio & CGAGCATAGGCAGAACTTAC \\
RP & /Bio/GAATGACTCGCTCTTACGAC \\
SCA2.1 & GAATGACTCGCTCTTACGAC \\
\hline \hline
\end{tabular}

$\mathrm{N}$ represents random base; /Bio/ represents biotin modification 


\subsubsection{SELEX strategy}

The isolation of aptamers was carried out via a parallel-and-serial selection strategy. The whole aptamer isolation process consisted of five (ethylone and butylone) or nine ( $\alpha$ PVP) rounds of parallel selection and two cycles of serial selection. Detailed information regarding the conditions for each round of selection are listed in Table 6-2. Since each sequence has a low copy number at the beginning of SELEX, high target concentrations were used during the first few rounds to ensure retention of all possible target binders. Moreover, counter-target concentrations were initially kept low to remove high-affinity interferent binders while avoiding loss of target binders. In later rounds of SELEX, selection stringency was increased by decreasing the target concentration and increasing the counter-target concentration; this would yield aptamers with high target affinity and specificity. The counter-SELEX protocol was rationally designed to encompass known and commonly-observed interferents found in seized drug samples, including cutting agents (e.g., caffeine and acetaminophen), adulterants (e.g., procaine, lidocaine, and promazine), other illicit drugs (e.g., cocaine and methamphetamine), or structurallyrelated non-cathinone molecules (e.g., amphetamine, pseudoephedrine and ephedrine) to ensure that the isolated aptamer did not bind to them.

\subsubsection{Parallel selection}

Parallel selection began with three initial pools consisting of 1 nmole DNA library (Table 6-1) for each of the three different selection targets ( $\alpha$-PVP, ethylone, butylone). From Round P2 to P9, 300 pmole of enriched library pool for each target from the previous round were employed in the subsequent round. Positive selection was performed with progressively decreasing target concentrations (Round P1: $1 \mathrm{mM}$, Rounds P2-P3: 
$500 \mu \mathrm{M}$, Rounds P4-P5: $250 \mu \mathrm{M}$ for $\alpha$-PVP, ethylone and butylone; Rounds P6-P9: 100 $\mu \mathrm{M}$ for $\alpha$-PVP only) to increase selection stringency for the enrichment of strong binders. For every round after the first, counter-selection was performed prior to positive selection in order to eliminate non-specific binders. The number of counter-targets and their concentrations were progressively increased during the selection process to increase selection stringency. Specifically, from Rounds P2-P5 for the ethylone and butylone pools and Rounds P2-P8 for the $\alpha$-PVP pool, we used the following counter-selection strategy: $100 \mu \mathrm{M}$ cocaine for Round $\mathrm{P} 2$, a mixture of $100 \mu \mathrm{M}$ cocaine and $100 \mu \mathrm{M}$ procaine for Round $\mathrm{P} 3$, and a mixture of $100 \mu \mathrm{M}$ cocaine, $100 \mu \mathrm{M}$ procaine, and $100 \mu \mathrm{M}$ lidocaine for Rounds P4-P8. For Round P9 for the $\alpha$-PVP pool, $300 \mu \mathrm{M}$ each of cocaine, procaine, and lidocaine was employed consecutively for counter selection (Table 6-2).

\subsubsection{Serial selection}

We combined 100 pmole each from the P5 parallel pools for ethylone and butylone and the P9 parallel pool for $\alpha$-PVP to generate the initial pool for serial selection. Two cycles of serial selection (Cycle 1 comprising Rounds S1-S3, Cycle 2 comprising Rounds S4-S6) were performed to specifically isolate cross-reactive aptamers. For each cycle, positive selection was performed by alternating the selection target (Rounds S1 \& S4: butylone, Rounds S2 \& S5: ethylone, and Rounds S3 \& S6: $\alpha$-PVP). Target concentration was maintained at $100 \mu \mathrm{M}$ for each round of serial selection for maximum stringency. In the first cycle of serial selection, counter-SELEX was performed prior to each round of positive selection by consecutively challenging the pool with $500 \mu \mathrm{M}$ each of cocaine, procaine, and lidocaine, while in the second cycle of serial selection, counterSELEX was performed by sequentially challenging with a mixture of $500 \mu \mathrm{M}$ each of 
ephedrine, pseudoephedrine, acetaminophen, methamphetamine, amphetamine; then with a mixture of $1 \mathrm{mM}$ each of cocaine, procaine, and lidocaine; and finally with $500 \mu \mathrm{M}$ promazine (Table 6-2).

Table 6-2. Detailed information regarding the conditions for each round of SELEX.

\begin{tabular}{|c|c|c|c|c|c|c|}
\hline & $\begin{array}{c}\text { Round } \\
\#\end{array}$ & $\begin{array}{l}\text { Pool size } \\
\text { (pmole) }\end{array}$ & & Counter-targets & & $\begin{array}{c}\text { Targets } \\
(\mu \mathrm{M})\end{array}$ \\
\hline \multirow{19}{*}{ 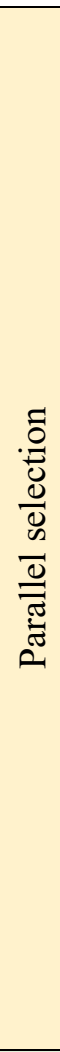 } & \multirow{3}{*}{$\mathrm{P} 1$} & 1000 & \multirow{3}{*}{\multicolumn{3}{|c|}{$\mathrm{N} / \mathrm{A}$}} & Butylone (1000) \\
\hline & & 1000 & & & & Ethylone (1000) \\
\hline & & 1000 & & & & $\alpha$-PVP $\quad(1000)$ \\
\hline & \multirow{3}{*}{$\mathrm{P} 2$} & 358 & \multirow{3}{*}{\multicolumn{3}{|c|}{$\operatorname{COC}(100 \mu \mathrm{M})$}} & Butylone (500) \\
\hline & & 346 & & & & Ethylone (500) \\
\hline & & 368 & & & & $\alpha$-PVP $\quad(500)$ \\
\hline & \multirow{3}{*}{$\mathrm{P} 3$} & 321 & \multirow{3}{*}{\multicolumn{3}{|c|}{ Mixture of COC \& PRC (100 $\mu \mathrm{M}$ each $)$}} & Butylone (500) \\
\hline & & 362 & & & & Ethylone (500) \\
\hline & & 345 & & & & $\alpha-P V P \quad(500)$ \\
\hline & \multirow{3}{*}{$\mathrm{P} 4$} & 319 & \multirow{3}{*}{\multicolumn{3}{|c|}{ Mixture of COC, PRC, \& LDC (100 $\mu \mathrm{M}$ each $)$}} & Butylone (250) \\
\hline & & 367 & & & & Ethylone (250) \\
\hline & & 292 & & & & $\alpha$-PVP $\quad(250)$ \\
\hline & \multirow{3}{*}{ P5 } & 300 & \multirow{3}{*}{\multicolumn{3}{|c|}{ Mixture of COC, PRC, \& LDC (100 $\mu \mathrm{M}$ each $)$}} & Butylone (250) \\
\hline & & 300 & & & & Ethylone (250) \\
\hline & & 300 & & & & $\alpha$-PVP $\quad(250)$ \\
\hline & P6 & 300 & Mixture of $\mathrm{COC}$ & PRC, \& LDC (100 & $\mu \mathrm{M}$ each) & $\alpha$-PVP $\quad(100)$ \\
\hline & $\mathrm{P} 7$ & 300 & Mixture of $\mathrm{COC}$ & , PRC, \& LDC (100 & $\mu \mathrm{M}$ each) & $\alpha$-PVP $\quad(100)$ \\
\hline & P8 & 300 & Mixture of $\mathrm{COC}$ & PRC, \& LDC (100 & $\mu \mathrm{M}$ each) & $\alpha-P V P \quad(100)$ \\
\hline & P9 & 300 & $\begin{array}{c}\text { COC } \\
(300 \mu \mathrm{M})\end{array}$ & $\begin{array}{c}\text { PRC } \\
(300 \mu \mathrm{M})\end{array}$ & $\begin{array}{c}\text { LDC } \\
(300 \mu \mathrm{M})\end{array}$ & $\alpha-P V P \quad(100)$ \\
\hline \multirow{6}{*}{ 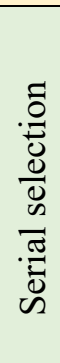 } & S1 & $300 *$ & \multirow{3}{*}{$\begin{array}{c}\mathrm{COC} \\
(500 \mu \mathrm{M})\end{array}$} & \multirow{3}{*}{$\begin{array}{c}\text { PRC } \\
(500 \mu \mathrm{M})\end{array}$} & \multirow{3}{*}{$\begin{array}{c}\text { LDC } \\
(500 \mu \mathrm{M})\end{array}$} & Butylone (100) \\
\hline & S2 & 300 & & & & Ethylone (100) \\
\hline & $\mathrm{S} 3$ & 300 & & & & $\alpha$-PVP $\quad(100)$ \\
\hline & S4 & 300 & \multirow{3}{*}{$\begin{array}{l}\text { Mixture of EPH, } \\
\text { PSE, ACM, } \\
\text { METH, \& AMP } \\
(500 \mu \mathrm{M} \text { each })\end{array}$} & \multirow{3}{*}{$\begin{array}{l}\text { Mixture of COC, } \\
\text { PRC, \& LDC } \\
(1000 \mu \mathrm{M} \text { each })\end{array}$} & \multirow{3}{*}{$\begin{array}{l}\text { Promazine } \\
(500 \mu \mathrm{M})\end{array}$} & Butylone (100) \\
\hline & $\mathrm{S} 5$ & 300 & & & & Ethylone (100) \\
\hline & S6 & 300 & & & & $\alpha$-PVP $\quad(100)$ \\
\hline
\end{tabular}

Consists of 100 pmole each of pool enriched with butylone (Round P5), ethylone (Round P5) and $\alpha$-PVP (Round P9).

Acetaminophen (ACM), amphetamine (AMP), cocaine (COC), ephedrine (EPH), lidocaine (LDC), methamphetamine (METH), procaine (PRC), pseudoephedrine (PSE). 


\subsubsection{SELEX procedure}

The isolation of aptamers was carried out following a previously-reported libraryimmobilized SELEX protocol. ${ }^{61}$ The initial single-stranded DNA library used for SELEX consisted of approximately $6 \times 10^{14}$ oligonucleotides. Each library strand is stem-loop structured and 73 nucleotides in length, with a randomized 30 nucleotide loop flanked in turn by a pair of 8-nt stem-forming sequences and two primer-binding regions (Table 6-1, DNA library). For each round of SELEX, the library/pool was mixed with biotinylated capture strands (Table 6-1, cDNA-bio) at a molar ratio of 1:5 in selection buffer (10 mM Tris- $\mathrm{HCl}, 0.5 \mathrm{mM} \mathrm{MgCl} 2,20 \mathrm{mM} \mathrm{NaCl}, \mathrm{pH} 7.4)$, heated at $95{ }^{\circ} \mathrm{C}$ for 10 min, and cooled at room temperature for over $30 \mathrm{~min}$ to ensure hybridization between library and capture strands. A micro-gravity column was prepared by adding $250 \mu \mathrm{L}$ of streptavidin-coated agarose beads followed by three washes with $250 \mu \mathrm{L}$ of selection buffer. $250 \mu \mathrm{L}$ of library solution was then flowed through the micro-gravity column three times in order to conjugate the library to the agarose beads (Figure 6-1A). The column was subsequently washed 10 times with selection buffer. Then, $250 \mu \mathrm{L}$ of target ( $\alpha$-PVP, ethylone or butylone) dissolved in selection buffer was added to the column. Library molecules that bound to the target underwent a conformational change, which caused them to detach themselves from the biotinylated cDNA into solution (Figure 6-1B). The eluent containing these strands was collected. This process was repeated twice, and all eluents were combined $(750 \mu \mathrm{L}$ total). The resulting pool was concentrated via centrifugation using a $3 \mathrm{kDa}$ cut-off spin filter. The concentrated pool $(100 \mu \mathrm{L})$ was then mixed with $1 \mathrm{~mL}$ of GoTaq Hot Start Colorless Master Mix with $1 \mu \mathrm{M}$ forward primer (Table 6-1, FP) and $1 \mu \mathrm{M}$ biotinylated reverse primer (Table 6-1, RP-bio) to 
amplify the pool via PCR. Amplification was performed using a BioRad C1000 thermal cycler with conditions as follows: 2 min at $95{ }^{\circ} \mathrm{C} ; 13$ cycles of $95{ }^{\circ} \mathrm{C}$ for $15 \mathrm{~s}, 58{ }^{\circ} \mathrm{C}$ for $30 \mathrm{~s}$, and $72{ }^{\circ} \mathrm{C}$ for $45 \mathrm{~s}$; and 5 min at $72{ }^{\circ} \mathrm{C}$. The optimal number of amplification cycles was determined by performing pilot PCR to ensure sufficient amplification of enriched sequences without generating PCR-related artifacts. Amplification of the enriched pool and the absence of byproducts was confirmed using 3\% agarose gel electrophoresis. If byproducts with differing lengths from the original library strands were observed, the pool was purified with a $4 \%$ agarose gel and the 73 -nt products were recovered by silica column as reported previously. ${ }^{61}$ To generate single-stranded DNA from the resulting double-stranded PCR products, a fresh micro-gravity column was prepared containing $250 \mu \mathrm{L}$ streptavidin-coated agarose beads. The amplified pool was then flowed through the column three times to conjugate the pool to the beads. Afterwards, the column was washed six times with $250 \mu \mathrm{L}$ of separation buffer $(10 \mathrm{mM}$ Tris- $\mathrm{HCl}, 20 \mathrm{mM} \mathrm{NaCl}, \mathrm{pH}$ 7.4). The column was then capped, and $300 \mu \mathrm{L}$ of a $0.2 \mathrm{M} \mathrm{NaOH}$ solution was added to the column and incubated for $10 \mathrm{~min}$ to generate single-stranded DNA, after which the eluent was collected. An additional $100 \mu \mathrm{L}$ of $0.2 \mathrm{M} \mathrm{NaOH}$ was added to elute residual library strands from the column. Both eluents were combined and neutralized with $0.2 \mathrm{M}$ $\mathrm{HCl}$, and the pool was then concentrated via centrifugation with a $3 \mathrm{kDa}$ cut-off spin filter. For every round after the first, counter-SELEX was performed before the positive selection step. Specifically, the library-immobilized column was washed with $250 \mu \mathrm{L}$ of counter-target(s) in selection buffer to remove non-specific DNA strands. This process was repeated three times for Rounds P1-P3 and S1-S6, and ten times for Rounds P4-P5 and, in the case of $\alpha$-PVP, Rounds P6-P9. Afterwards, the column was washed 30 times 
with selection buffer to wash away non-specific binders in preparation for positive selection.
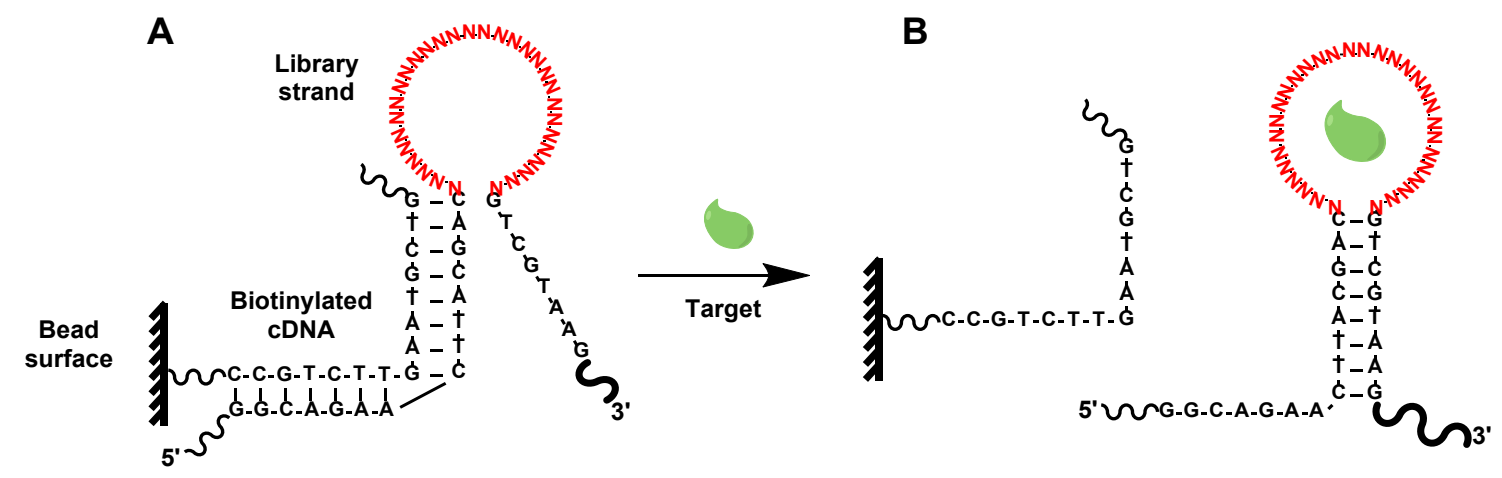

Figure 6-1. Scheme for library-immobilized SELEX. (A) Library molecules are immobilized on streptavidin-coated agarose beads via a biotinylated complementary DNA (cDNA) strand. (B) Strands that bind to the target are released into solution via target-induced strand displacement.

\subsubsection{Gel elution assay}

The enrichment, target affinity, specificity, and cross-reactivity of the pools collected after Rounds P5 (for $\alpha$-PVP, ethylone, and butylone), P9 (for $\alpha$-PVP only), S3, and S6 were evaluated using a modified version of a previously reported gel elution assay (Figure 6-2). ${ }^{34}$ Specifically, 50 pmole of enriched library (Figure 6-2A) was incubated with 250 pmole of biotinylated cDNA in $125 \mu \mathrm{L}$ of selection buffer, heated at $95^{\circ} \mathrm{C}$ for $10 \mathrm{~min}$, and cooled at room temperature over $30 \mathrm{~min}$ to anneal both strands and form cDNA-library complex (Figure 6-2B). Afterwards, a microcentrifugation column was prepared by adding $125 \mu \mathrm{L}$ of streptavidin-coated agarose beads. The cDNA-library complex was then added to the column and immobilized on the beads (Figure 6-2C), and the eluent was collected and recycled through the column twice more. The libraryimmobilized agarose beads were transferred into a microcentrifugation tube and washed five times by adding $625 \mu \mathrm{L}$ of selection buffer, incubating on an end-over-end rotator for $5 \mathrm{~min}$, followed by centrifugation and removal of the supernatant. The volume of the 
library-immobilized bead solution was adjusted to $150 \mu \mathrm{L}$ with selection buffer and aliquoted into 7 tubes $(20 \mu \mathrm{L} /$ tube). Afterwards, $50 \mu \mathrm{L}$ of target at a variety of final concentrations $(0,10,50,100,250,500$, or $1,000 \mu \mathrm{M})$ was added into each tube (Figure 6-2D). After rotating for $60 \mathrm{~min}$ on an end-over-end rotator at room temperature, the beads were settled by centrifugation and $40 \mu \mathrm{L}$ of the supernatant, which contained the target-eluted strands, was collected and set aside (Figure 6-2E). Meanwhile the leftover solution $(30 \mu \mathrm{L})$ (Figure 6-2F) was mixed with $50 \mu \mathrm{L}$ of a $95 \%$ formamide solution containing $10 \mathrm{mM}$ EDTA and incubated at $90{ }^{\circ} \mathrm{C}$ for $10 \mathrm{~min}$ to completely release all DNA strands from the beads (Figure 6-2G). The resulting solution contained both leftover target-eluted strands and non-target-eluted strands. We analyzed the target-eluted aptamer solution and formamide-treated library solution via $15 \%$ denaturing polyacrylamide gel electrophoresis (PAGE) and determined the concentrations of the strands based on standardized concentrations of ladder loaded in the gel. The elution percentage was calculated using the equation: $\theta=\frac{V_{1} \times c_{s}}{V_{2} \times c_{s}+V_{3} \times c_{b}} \times 100 \%$

where $\theta$ is the fraction of target-eluted strands, $c_{s}$ is the concentration of target-eluted strands, $c_{b}$ is the concentration of strands in the formamide solution, $V_{1}$ is the volume of solution before supernatant collection (estimated as $62 \mu \mathrm{L}$, with $\sim 8 \mu \mathrm{L}$ occupied by agarose beads), $\mathrm{V}_{2}$ is the volume of the collected supernatant containing target-eluted strands ( $40 \mu \mathrm{L}$ ), and $\mathrm{V}_{3}$ is the volume of solution after addition of formamide $(80 \mu \mathrm{L})$. A calibration curve was created by plotting the fraction of eluted strands against the employed target concentration. The resulting curve was fitted with the Langmuir equation to determine the dissociation constant $\left(\mathrm{K}_{\mathrm{D}}\right)$ of the enriched pool. The same protocol was 
used to determine the target cross-reactivity and specificity of the enriched pool for other synthetic cathinones (4-MMC, 4-FMC, MDPBP, MDPV, MePBP, methedrone, methylone, MPHP, naphyrone, pentylone, and pyrovalerone) or interferents (acetaminophen, amphetamine, caffeine, cocaine, ephedrine, lidocaine, methamphetamine, procaine, promazine, pseudoephedrine, and sucrose).

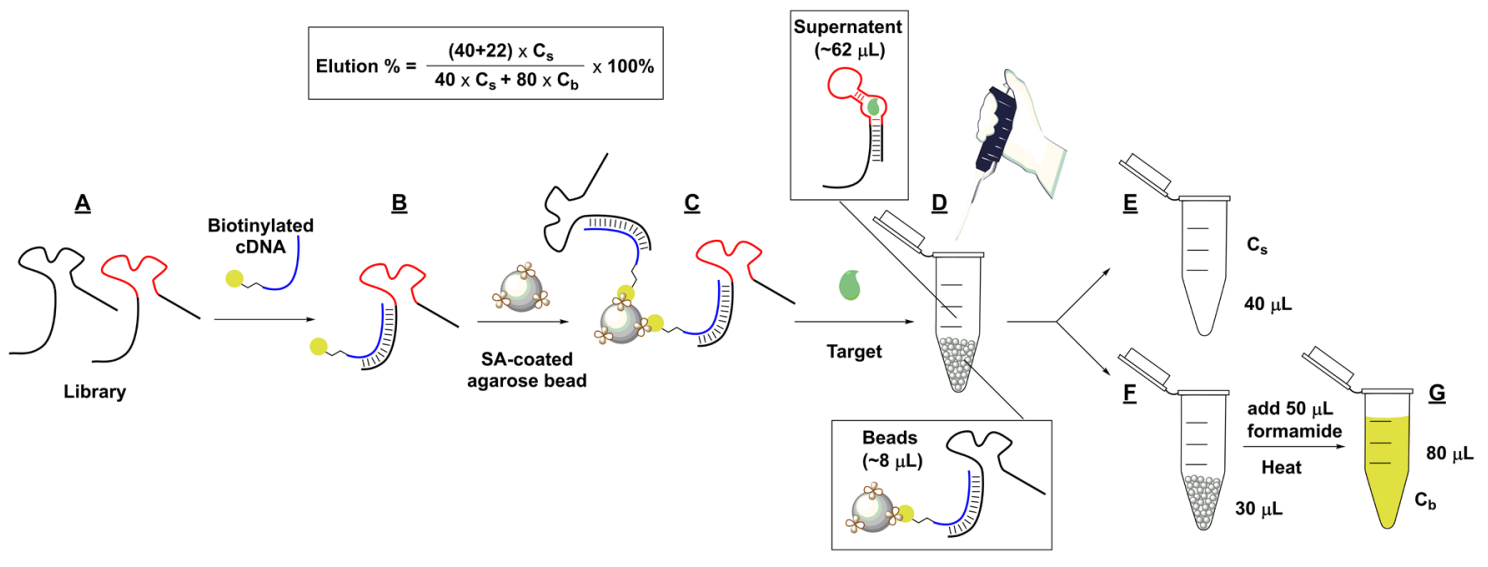

Figure 6-2. Scheme of the gel elution assay. (A) Library strands are hybridized with biotinylated cDNA to form (B) library-cDNA complex. (C) The resulting complex is immobilized on streptavidin-coated agarose beads. (D) The target is added the solution and (E) the supernatant containing the target-bound strands is collected and added to a separate tube. (F) The leftover beads are treated with formamide-EDTA and heat to $(\mathbf{G})$ release remaining non-binding strands from the beads. The quantity of DNA in the solutions obtained from $E$ and $G$ is determined via PAGE; the number of eluted strands is used to calculate the pool binding affinity and specificity using the boxed equation.

\subsubsection{High throughput sequencing (HTS) analysis of the aptamer isolation process}

HTS of the three final parallel pools and two serial pools was performed using Ion Torrent Sequencing. To prepare samples for sequencing, $1 \mu \mathrm{M}$ pool was mixed with GoTaq ${ }^{\circledR}$ Hot Start Colorless Master Mix, $1 \mu \mathrm{M}$ forward primer, and $1 \mu \mathrm{M}$ reverse primer with a final volume of $100 \mu \mathrm{L}$. PCR was then performed with the same PCR conditions as described in 'SELEX Procedure'. Then $40 \mu \mathrm{L}$ of PCR product was added into $16 \mu \mathrm{L}$ of ExoSAP-IT ${ }^{\mathrm{TM}}$ reagent in an ice bath. The mixture was then incubated at $37^{\circ} \mathrm{C}$ for 15 min to degrade remaining primers and nucleotides, followed by incubation at $80^{\circ} \mathrm{C}$ for 15 
min to inactivate ExoSAP-IT reagent. HTS was performed at FIU DNA Core Facility using an Ion Personal Genome Machine System with an Ion 318 v2 chip (ThermoFisher Scientific). Upon obtaining the sequencing data, the primer sequences were trimmed by cutadapt $^{234}$ and the population of sequences from each pool were calculated using FASTAptamer. ${ }^{235}$ Members of the SCA2.1 family are defined as sequences containing "AGTGGGGTTCGGGTGGAGTT" in any position in the 30-nt random region.

\subsubsection{Cloning and sequencing}

Cloning and sequencing of the final enriched pool was performed using a previously reported protocol. ${ }^{61}$ Briefly, the enriched sequences from the final pool (Round S6) were amplified by PCR with unlabeled forward and reverse primers (Table 6-1, FP and RP) using the same program as described above in 'SELEX procedure'. At the end of the amplification protocol, an additional 30 min extension step was performed at $72{ }^{\circ} \mathrm{C}$ to add a poly-A tail. Amplicons were cloned into a plasmid vector and transformed into $E$. coli cells using the TOPO TA cloning kit (Invitrogen) according to the supplier recommendations. The plasmids from 50 randomly picked colonies were extracted using a PureLink Quick Plasmid Miniprep Kit (Invitrogen) and sequenced via Sanger Sequencing at the Florida International University DNA Core Facility. After removing the sequences of the plasmid and primers, the aptamer sequences were aligned with BioEdit software to determine the consensus sequence.

\subsubsection{Isothermal titration calorimetry (ITC)}

ITC was performed using a MicroCal ITC200 instrument (Malvern). All ITC experiments were carried out using the following protocol at $23{ }^{\circ} \mathrm{C}$. A solution containing the aptamer (final concentration $20 \mu \mathrm{M}$ ) was prepared in selection buffer $(10 \mathrm{mM}$ Tris- 
$\mathrm{HCl}, 0.5 \mathrm{mM} \mathrm{MgCl}_{2}, 20 \mathrm{mM} \mathrm{NaCl}, \mathrm{pH}$ 7.4) and loaded into the ITC sample cell. We then loaded different concentrations of synthetic cathinone $(350 \mu \mathrm{M}( \pm)$ - $\alpha$-PVP, $300 \mu \mathrm{M}( \pm)$ ethylone, $400 \mu \mathrm{M}( \pm)$-butylone, $300 \mu \mathrm{M}( \pm)$-MDPV, $225 \mu \mathrm{M}(-)-\mathrm{MDPV}$, or $400 \mu \mathrm{M}(+)$ MDPV) or interferent (1 $\mathrm{mM} \mathrm{( \pm )-amphetamine,} \mathrm{(+)-methamphetamine,} \mathrm{cocaine,}$ procaine, or (+)-ephedrine) in the same buffer into the syringe and titrated it into the cell, with an initial $0.4 \mu \mathrm{L}$ purge injection followed by 19 successive $2 \mu \mathrm{L}$ injections. A spacing of 180 seconds was used between each titration. The heat generated from each titration was recorded, and the binding stoichiometry $(\mathrm{N})$, enthalpy $(\Delta \mathrm{H})$, and $\mathrm{K}_{\mathrm{D}}$ were obtained by fitting the resulting titration curve with a single-site binding model (wherein the aptamer and synthetic cathinone are considered to be the receptor and ligand, respectively) using the MicroCal analysis kit integrated into Origin 7 software. All titrations involving racemic synthetic cathinone mixtures were also fitted with a modified two-site binding model to determine the binding parameters for each enantiomer. This model assumes that a receptor with two ligand-binding sites independently interacts with a ligand. Unlike the single-site binding model, the aptamer in the sample cell is considered as the ligand and the pair of synthetic cathinone enantiomers is considered as a receptor with two discrete binding sites. For example, titration of $300 \mu \mathrm{M}( \pm)-\mathrm{MDPV}-$ containing $150 \mu \mathrm{M}$ each of (-)-MDPV and (+)-MDPV - into $20 \mu \mathrm{M}$ SCA2.1 is treated as titration of $150 \mu \mathrm{M}$ of a receptor with two independent 'binding sites' into $20 \mu \mathrm{M}$ of ligand. The binding parameters of the first site (i.e. $\mathrm{N}_{1}, \mathrm{~K}_{\mathrm{Dl}}, \Delta \mathrm{H}_{1}$ ) reflect the binding of one of the enantiomers to the aptamer, while those for the second site (i.e. $\mathrm{N}_{2}, \mathrm{~K}_{\mathrm{D} 2}, \Delta \mathrm{H}_{2}$ ) reflect the binding of the other. 


\subsubsection{Characterization of $\mathrm{Cy} 7$ binding to SCA2.1}

$8 \mu \mathrm{L}$ of different concentrations of SCA2.1, $8 \mu \mathrm{L}$ of $20 \mu \mathrm{M} \mathrm{Cy} 7$, and $64 \mu \mathrm{L}$ of reaction buffer (final concentration $10 \mathrm{mM}$ Tris- $\mathrm{HCl}, 0.5 \mathrm{mM} \mathrm{MgCl}, 20 \mathrm{mM} \mathrm{NaCl}$, $0.01 \%$ Tween 20 , and $1 \%$ DMSO, $\mathrm{pH} 7.4$ ) were mixed in the wells of a transparent flatbottomed 384-well plate. UV-vis spectra were immediately recorded from 450-900 nm using a Tecan Infinite M1000 PRO microplate reader at room temperature. The absorbance value at $775 \mathrm{~nm}$ ( $\lambda_{\max }$ of $\mathrm{Cy} 7$ monomer) was plotted against the concentration of added aptamer. $\mathrm{K}_{\mathrm{D}}$ was estimated by non-linear fitting using the Langmuir equation.

\subsubsection{Cy7-displacement assay for colorimetric detection of synthetic cathinones}

$8 \mu \mathrm{L}$ of SCA2.1 (final concentration: $3 \mu \mathrm{M}$ ), $8 \mu \mathrm{L}$ of Cy7 (final concentration $2 \mu \mathrm{M}$ ), $8 \mu \mathrm{L}$ of varying concentrations of target ( $\alpha-\mathrm{PVP}$, butylone, or ethylone), and $56 \mu \mathrm{L}$ of reaction buffer (final concentration $10 \mathrm{mM}$ Tris- $\mathrm{HCl}, 0.5 \mathrm{mM} \mathrm{MgCl}_{2}, 20 \mathrm{mM} \mathrm{NaCl}$, $0.01 \%$ Tween $20,1 \%$ DMSO, $\mathrm{pH} 7.4$ ) were mixed in the wells of a transparent flatbottomed 384-well plate. UV-vis spectra were immediately recorded from 450-900 nm using a Tecan microplate reader at room temperature. The absorbance ratio between 670 $\mathrm{nm}$ and $775 \mathrm{~nm}\left(\mathrm{~A}_{670} / \mathrm{A}_{775}\right)$ was calculated for each sample, and signal gain was calculated by $\left(\mathrm{R}-\mathrm{R}_{0}\right) / \mathrm{R}_{0}$, where $\mathrm{R}_{0}$ and $\mathrm{R}$ represent $\mathrm{A}_{670} / \mathrm{A}_{775}$ without and with target, respectively. Using the same protocol, the cross-reactivity and specificity of the assay were tested with other synthetic cathinones (naphyrone, MDPV, pentylone, methylone, 4MMC, 4-FMC, 3-FMC, methcathinone and cathinone) and interferents (amphetamine, methamphetamine, cocaine, pseudoephedrine, ephedrine, procaine, lidocaine, benzocaine, caffeine, acetaminophen, and sucrose) at a concentration of $50 \mu \mathrm{M}$. Crossreactivity was calculated using the signal gain of $50 \mu \mathrm{M}$ ethylone as $100 \%$. For visual 
synthetic cathinone detection, the assay was performed using the same protocol but with higher concentrations of SCA2.1 $(5 \mu \mathrm{M})$ and $\mathrm{Cy} 7(3.5 \mu \mathrm{M})$ with $50 \mu \mathrm{M}$ of the aformentioned synthetic cathinones or interferents. Samples were loaded into a white flat-bottomed 384-well plate. Photographs of the samples were taken using a digital camera immediately after mixing all reaction components.

\subsection{Results and discussion}

\subsubsection{Choosing targets for selection}

Synthetic cathinones share the same $\beta$-keto phenethylamine chemical core structure and typically differ at four substituent sites (Figure 6-3A, core structure). Performing selection directly against this 'bare core' (i.e. 2-amino-propiophenone, also known as cathinone) may yield aptamers with high affinity towards cathinone, but there is no guarantee that such aptamers will bind other synthetic cathinones that have different substituents on the core structure. This is supported by previous studies which showed that small-molecule-binding aptamers isolated for a single target often have lower or no affinity for compounds with additional/differing substituents. ${ }^{25,110}$ We rationalized that performing SELEX with a set of structurally-similar, yet sufficiently diverse, targets would create selection pressure for isolating aptamers that tolerate variations at all such sites, greatly increasing the likelihood that the isolated aptamer will have high crossreactivity to this family as a whole. Thus, to isolate class-specific synthetic cathinonebinding aptamers, we selected three targets that share the same core structure but have variations at all of the substitution sites that are typically modified in this family: $\alpha$ pyrrolidinovalerophenone ( $\alpha$-PVP), ethylone, and butylone (Figure 6-3A). 


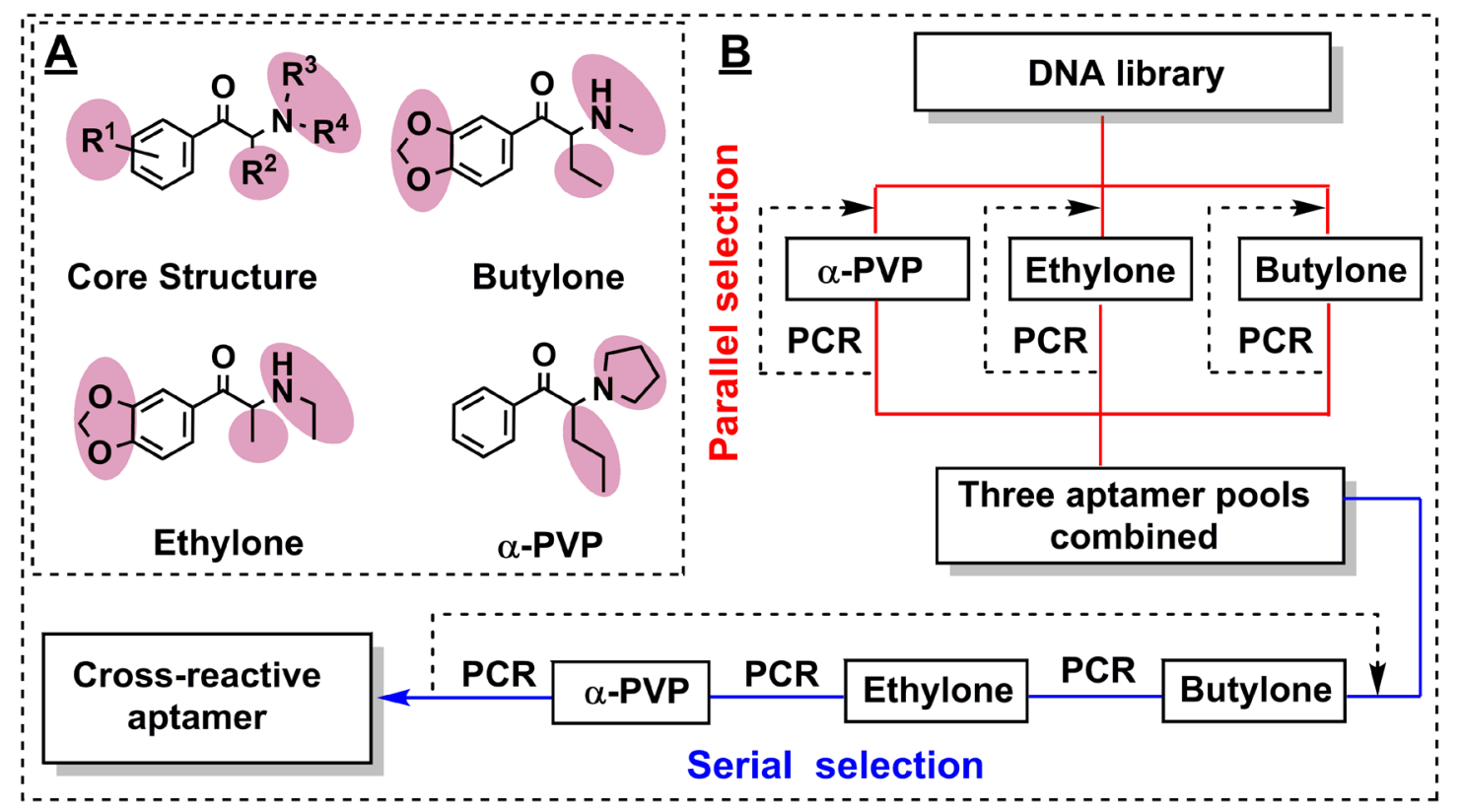

Figure 6-3. Isolation of a class-specific aptamer using a parallel-and-serial SELEX strategy. (A) The core structure of synthetic cathinones and the three targets chosen for parallel-and-serial SELEX, with substituent moieties shaded in red. (B) Schematic diagram of parallel-and-serial SELEX. Each of the three targets was subjected to parallel SELEX to enrich for broadly synthetic cathinone-specific aptamers (top), after which the resulting pools were combined and subjected to serial SELEX screening (bottom) to eliminate target-specific binders while retaining those aptamers with broad cross-reactivity for this drug class.

\subsubsection{Parallel selection}

Parallel selection was performed using three different initial library pools, with one pool being challenged with $\alpha$-PVP, one with ethylone, and one with butylone (Figure 6-3B, top). Presumably, challenging the parallel pools with individual targets enriches all sequences binding to the target, including those that are also cross-reactive to other synthetic cathinones. In contrast, challenging a single pool with a mixture of targets may lead to loss cross-reactive aptamers. This is evidenced by a previous study which demonstrated that performing SELEX with a mixture of four targets yielded aptamers that specifically bind to just one of the four. ${ }^{37}$ This may be attributable to competitive binding among these targets. 

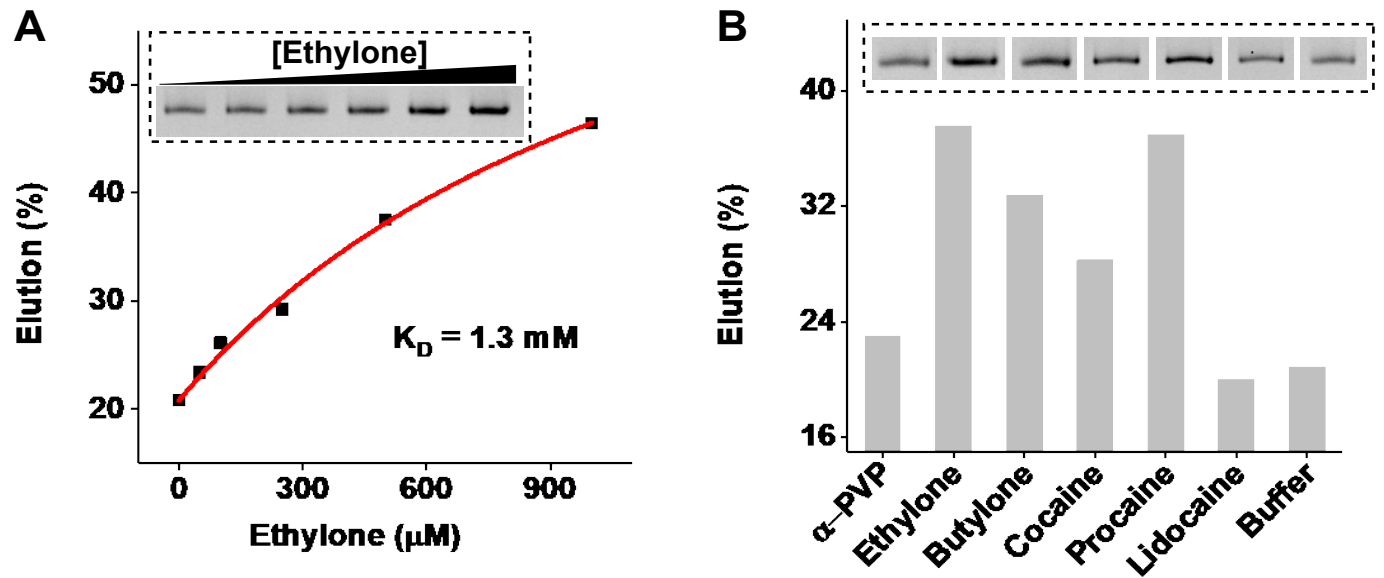

Figure 6-4. Determination of the target-binding affinity, cross-reactivity, and specificity of the round P5 ethylone pool via gel-elution assay. (A) Polyacrylamide gel electrophoresis (PAGE) results depict the target elution profile, with lanes representing samples of the pool eluted with 0 , $50,100,250,500$, or $1,000 \mu \mathrm{M}$ ethylone (left to right). The percent of target-eluted pool was plotted against the concentration of ethylone employed for elution to determine the binding affinity of the enriched pool. (B) PAGE analysis (top) of elution with $500 \mu \mathrm{M}$ synthetic cathinones ( $\alpha$-PVP, ethylone, butylone) or interferents (cocaine, procaine, lidocaine) was used to measure cross-reactivity and specificity of the enriched P5 pool.
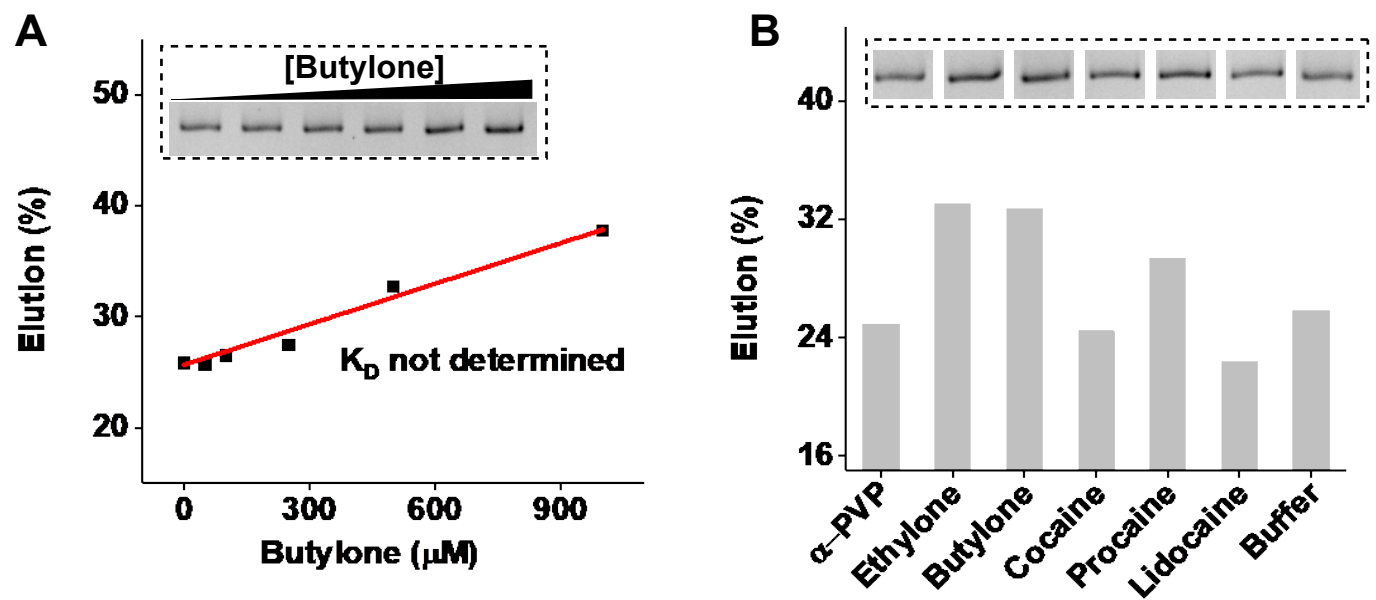

Figure 6-5. Determination of the target-binding affinity, cross-reactivity, and specificity of the round P5 butylone pool via gel-elution assay. (A) PAGE results depict the target elution profile, with lanes representing samples of the pool eluted with $0,50,100,250,500$, or $1,000 \mu \mathrm{M}$ butylone (left to right). The percent of target-eluted pool was plotted against the concentration of butylone employed for elution to determine the binding affinity of the enriched pool. (B) PAGE analysis (top) of elution with $500 \mu \mathrm{M}$ synthetic cathinones ( $\alpha$-PVP, ethylone, butylone) or interferents (cocaine, procaine, lidocaine) was used to measure cross-reactivity and specificity of the enriched P5 pool. 

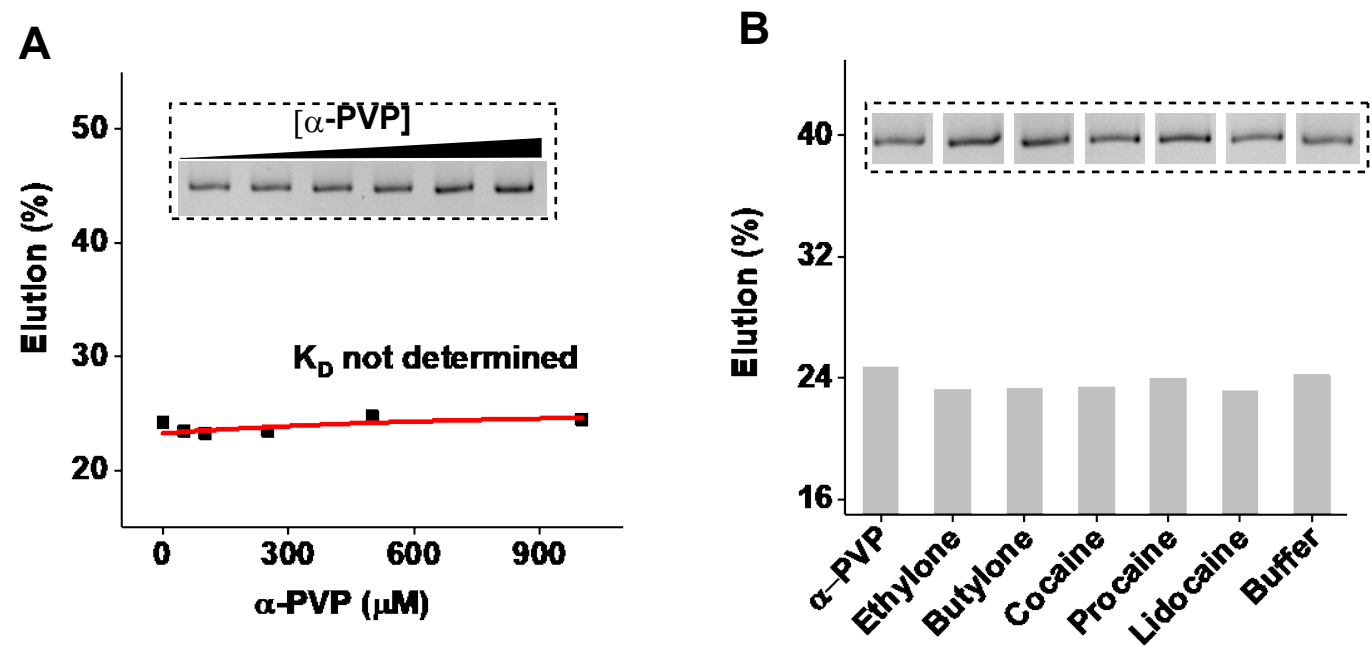

Figure 6-6. Determination of the target-binding affinity, cross-reactivity, and specificity of the round P5 $\alpha$-PVP pool via gel-elution assay. (A) PAGE results depict the target elution profile, with lanes representing samples of the pool eluted with $0,50,100,250,500$, or 1,000 $\mu \mathrm{M} \alpha-\mathrm{PVP}$ (left to right). The percent of target-eluted pool was plotted against the concentration of $\alpha-P V P$ employed for elution to determine the binding affinity of the enriched pool. (B) PAGE analysis (top) of elution with $500 \mu \mathrm{M}$ synthetic cathinones ( $\alpha$-PVP, ethylone, butylone) or interferents (cocaine, procaine, lidocaine) was used to measure target-cross-reactivity and specificity of the enriched P5 pool.
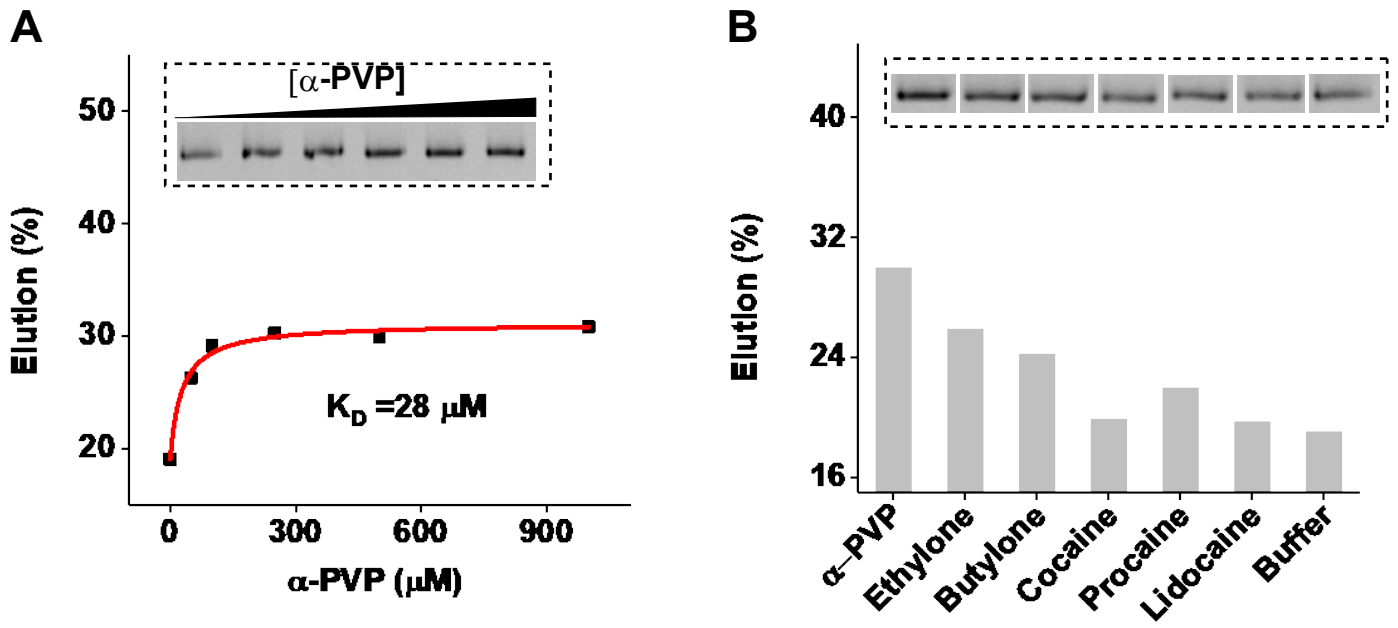

Figure 6-7. Determination of the target-binding affinity, cross-reactivity, and specificity of the round P9 $\alpha$-PVP pool via gel-elution assay. (A) PAGE results depict the target elution profile, with lanes representing samples of the pool eluted with $0,50,100,250,500$, or 1,000 $\mu \mathrm{M} \alpha$-PVP (left to right). The percent of target-eluted pool was plotted against the concentration of $\alpha$-PVP employed for elution to determine the binding affinity of the enriched pool. (B) PAGE analysis (top) of elution with $500 \mu \mathrm{M}$ synthetic cathinones ( $\alpha$-PVP, ethylone, butylone) or interferents (cocaine, procaine, lidocaine) was used to measure target-cross-reactivity and specificity of the enriched P9 pool. 
To perform parallel selection, we employed a library pool consisting of $\sim 6 \times 10^{14}$ unique oligonucleotide sequences, which form an 8-bp stem and a 30-nt random loop as the putative target-binding domain (Figure 6-1A). During the first round, each initial library pool was challenged with $1 \mathrm{mM}$ target, and eluted strands were collected and amplified for the next round of selection. To further establish class-specificity, from the second round onward we performed counter-SELEX prior to the positive selection step to remove aptamers binding to structurally-similar interferents (e.g. cocaine, procaine, lidocaine) that have the same functional groups or partial structural features as our targets. In round two, counter-SELEX was first performed for each pool against $100 \mu \mathrm{M}$ cocaine, followed by positive selection with $500 \mu \mathrm{M}$ target, as reducing target concentration increases selection stringency ${ }^{62-64}$ In the third round, a mixture of $100 \mu \mathrm{M}$ cocaine and $100 \mu \mathrm{M}$ procaine was used for counter-SELEX, with the same target concentration for positive selection. In rounds four and five, counter-SELEX was performed against cocaine, procaine, and lidocaine (each at a concentration of $100 \mu \mathrm{M}$ ) in a mixture, with $250 \mu \mathrm{M}$ target used for the positive selection step.

After the fifth round, a gel-elution assay (see methods) was performed to determine the target-binding affinity of each pool for its respective target. We observed that the fraction of eluted library increased with increasing target concentrations for the ethylone and butylone pools (Figure 6-4A and Figure 6-5A), showing that aptamers binding to these targets had been enriched through parallel selection. In contrast, target elution remained low for the $\alpha$-PVP pool (Figure 6-6A) regardless of the employed concentration of target, which indicated that the pool was not yet enriched. We further determined the cross-reactivity and specificity of the three pools via the gel-elution assay. The enriched 
ethylone and butylone pools were able to bind to both ethylone and butylone, but not to $\alpha-\mathrm{PVP}$, which indicated that the population of cross-reactive aptamers was relatively low. These pools also showed some affinity to procaine, with the ethylone pool also binding to cocaine. Neither pool displayed any affinity for lidocaine (Figure 6-4B and Figure 6-5B). In contrast, the $\alpha$-PVP pool showed no affinity for any of the targets or counter-targets (Figure 6-6B).

Given that the $\alpha$-PVP pool was not yet enriched, we performed additional rounds of selection. From rounds six to eight, we used the same counter-target mixture of $100 \mu \mathrm{M}$ cocaine, procaine, and lidocaine from round 5 with a further-reduced $\alpha$-PVP concentration of $100 \mu \mathrm{M}$ for positive selection. For the ninth round, we used the same $\alpha$ PVP concentration but with counter-selection performed with $300 \mu \mathrm{M}$ of each of the three counter-targets in a consecutive manner. After the ninth round, we performed the gel-elution assay with the enriched pool and observed a clear target concentrationdependent elution profile for $\alpha-\mathrm{PVP}$, with an estimated dissociation constant $\left(\mathrm{K}_{\mathrm{D}}\right)$ of 28 $\mu \mathrm{M}$ (Figure 6-7A). Notably, only $30 \%$ of the library was eluted, even in the presence of 1 $\mathrm{mM} \alpha$-PVP, which implied that there was just a small population of binders in the pool. We also determined that this pool displayed affinity to ethylone and butylone but was less responsive towards the various interferents (Figure 6-7B), which can be attributed to the fact that more rounds of counter-SELEX were performed. Given that the pools enriched with individual targets also cross-reacted to other targets, it seemed likely that those pools contained cross-reactive aptamers. We believed that if parallel selection was continued, highly target-specific aptamers would have begun to dominate the pool, therefore we terminated parallel selection before this could occur. 

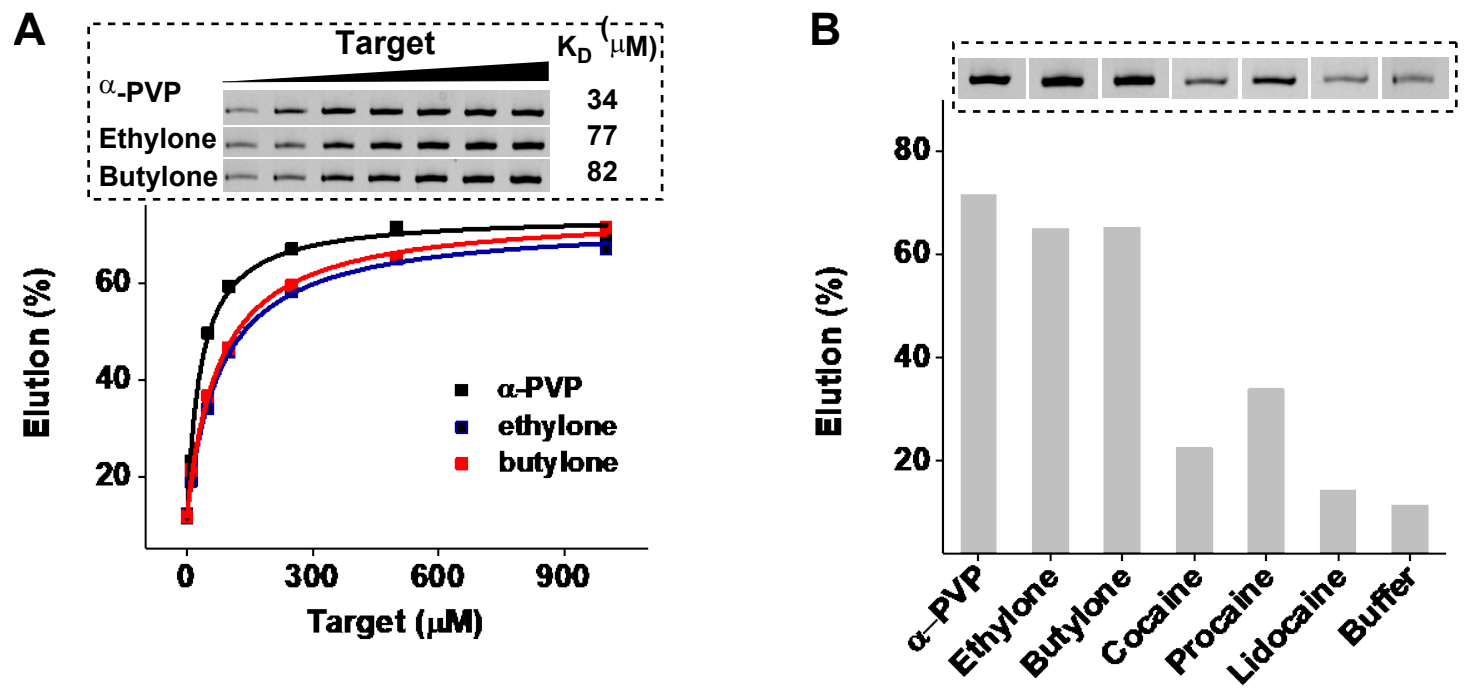

Figure 6-8. Determination of the target-binding affinity, cross-reactivity and specificity of the round S3 pool via a gel elution assay. (A) PAGE results depict the target elution profile with lanes representing samples of the pool eluted with $0,1050,100,250,500$, or $1,000 \mu \mathrm{M}$ (left to right) of $\alpha$-PVP, ethylone, or butylone. The percent of target-eluted pool was plotted against the concentration of target used for elution to determine the binding affinity of the enriched pool. (B) PAGE analysis of enriched pool eluted with $500 \mu \mathrm{M}$ synthetic cathinones ( $\alpha$-PVP, ethylone, butylone) or interferents (cocaine, procaine, lidocaine) was used to measure target-crossreactivity and specificity of the enriched S3 pool.
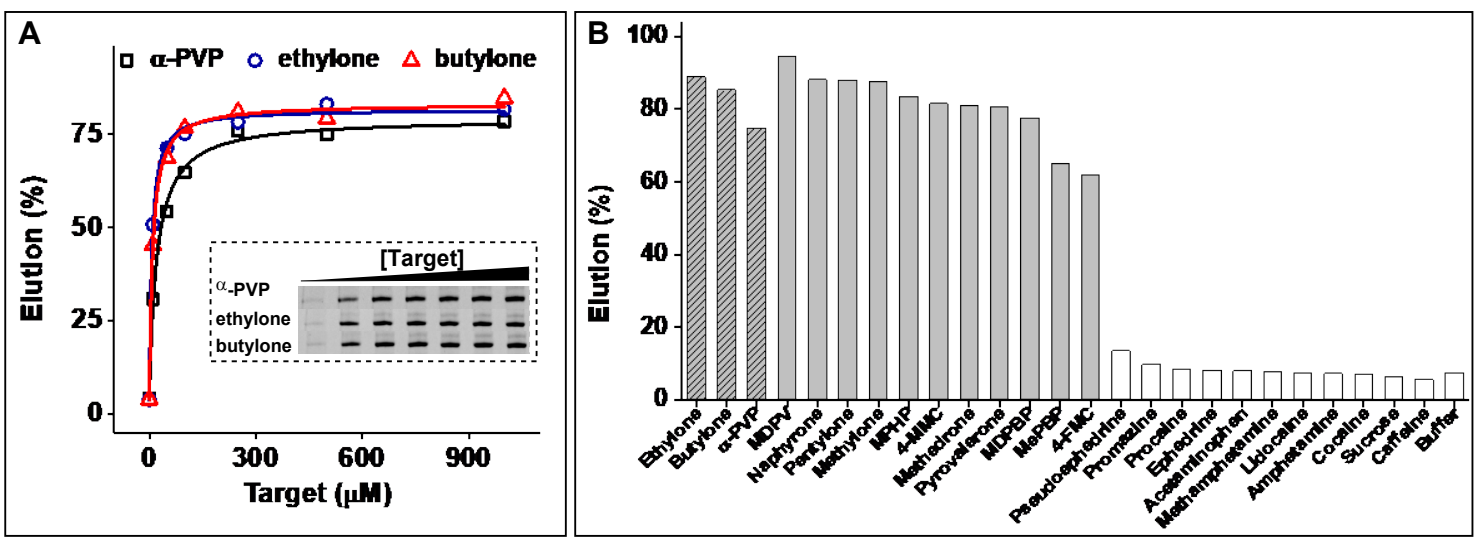

Figure 6-9. Characterization of the affinity and specificity of the final enriched pool via a gelelution assay. (A) PAGE results depict the target elution profile, with lanes representing samples of the pool eluted with $0,10,50,100,250,500$, or $1,000 \mu \mathrm{M}$ (from left to right) $\alpha$-PVP, ethylone, or butylone. The percent of target-eluted pool was plotted against the concentration of target to determine the binding affinity of the enriched pool. (B) Percent elution values for 14 synthetic cathinones and 11 interferents at a concentration of $50 \mu \mathrm{M}$ and buffer alone. 


\subsubsection{Serial selection}

We then performed serial selection to enrich cross-reactive aptamers and exclude those specific to individual targets (Figure 6-3B, bottom). We combined all three enriched parallel pool as a starting library. For each cycle of serial selection, we challenged the combined pool with each target sequentially for a total of three rounds of selection using butylone (first round), ethylone (second round), and $\alpha$-PVP (third round). In each round, we first performed counter-SELEX with $500 \mu \mathrm{M}$ cocaine, procaine, and lidocaine sequentially followed by positive selection with $100 \mu \mathrm{M}$ target. After the first cycle of serial selection against all three targets, we performed the gel-elution assay to determine the cross-reactivity and specificity of the resulting pool. We observed that the cross-reactivity towards ethylone and butylone had substantially increased $\left(\mathrm{K}_{\mathrm{D}}=82 \mu \mathrm{M}\right.$ and $77 \mu \mathrm{M}$, respectively) relative to the individual pools obtained for these targets at the end of parallel selection, while affinity towards $\alpha$-PVP was essentially unchanged $\left(\mathrm{K}_{\mathrm{D}}=\right.$ $34 \mu \mathrm{M}$ ) (Figure 6-8A). Importantly, this pool exhibited greatly improved specificity, with minimal affinity for cocaine and lidocaine and only a moderate response to procaine (Figure 6-8B). We then performed a second cycle of serial selection with an identical selection procedure but with a three-stage counter-SELEX process entailing sequential screening against a mixture of $500 \mu \mathrm{M}$ each of ephedrine, pseudoephedrine, acetaminophen, methamphetamine, amphetamine, then a mixture of $1 \mathrm{mM}$ each of cocaine, procaine, and lidocaine, and finally with $500 \mu \mathrm{M}$ promazine. We believe that the inclusion of these additional counter-targets, which are similar in structure to synthetic cathinones and commonly encountered in seized substances, further enhance the specificity of the enriched pool. After this cycle, we again evaluated the pool binding 
affinity via the gel-elution assay (Figure 6-9A). Each individual target (at a concentration of $500 \mu \mathrm{M}$ ) eluted more than $70 \%$ of the pool. The pool affinity towards ethylone and butylone had increased by $\sim 10$-fold ( $\mathrm{K}_{\mathrm{D}}=6.9 \mu \mathrm{M}$ and $9.5 \mu \mathrm{M}$, respectively), whereas the affinity towards $\alpha-P V P$ only marginally increased $\left(K_{D}=21 \mu \mathrm{M}\right)$.

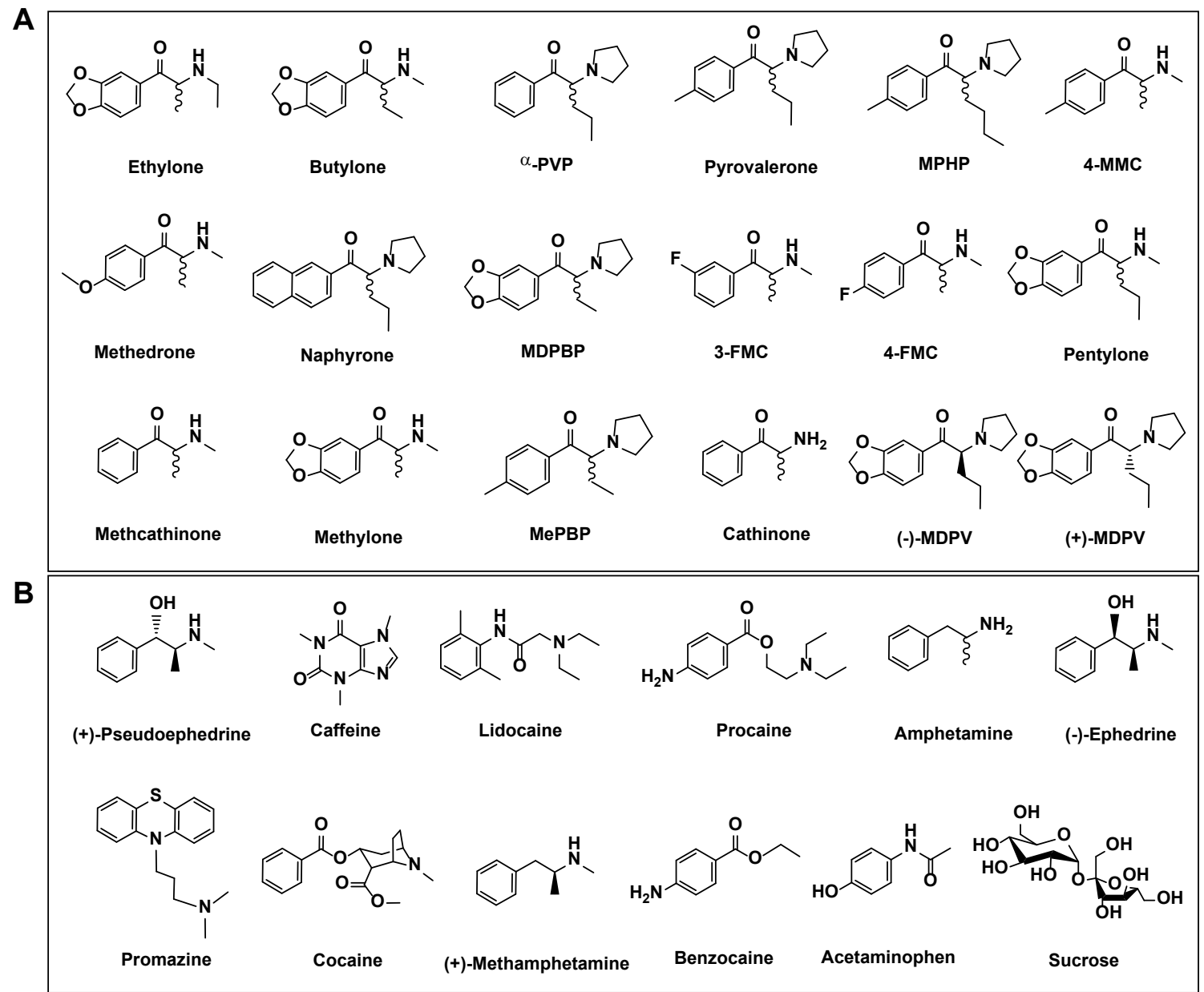

Figure 6-10. Chemical structures. The chemical structures of the (A) synthetic cathinones and (B) interferent compounds used in this work are shown. Note that racemic MDPV was also used in this work.

\subsubsection{Characterization and sequencing of the final serial pool}

We concluded that at this stage, the enriched pool largely comprised of cross-reactive synthetic-cathinone-binding aptamers. To confirm this, we used the gel-elution assay to 
test the cross-reactivity of this pool by challenging it with the three targets as well as 11 other synthetic cathinones (Figure $6-10 \mathrm{~A}$ ). All of them demonstrated $>60 \%$ target elution at a concentration of $50 \mu \mathrm{M}$ (Figure 6-9B). This shows that the aptamer could recognize the core structure of synthetic cathinones, while being tolerant even to side-chain substituents that were not encountered during SELEX. To evaluate the specificity of the enriched pool, we challenged the pool with $50 \mu \mathrm{M}$ of the counter targets and other potential interferents (Figure 6-10B) and found that none of them showed increased elution compared with buffer alone (Figure 6-9B). We therefore cloned and sequenced this final enriched pool. The pool was revealed to have low diversity, with 30 of the 50 clones having an identical sequence, which we named SCA2.1 (Table 6-3). Mfold ${ }^{82}$ predicts that SCA2.1 has a stem-loop structure with a 9-bp stem and a 28-nt loop in our selection buffer at room temperature (Figure 6-11).

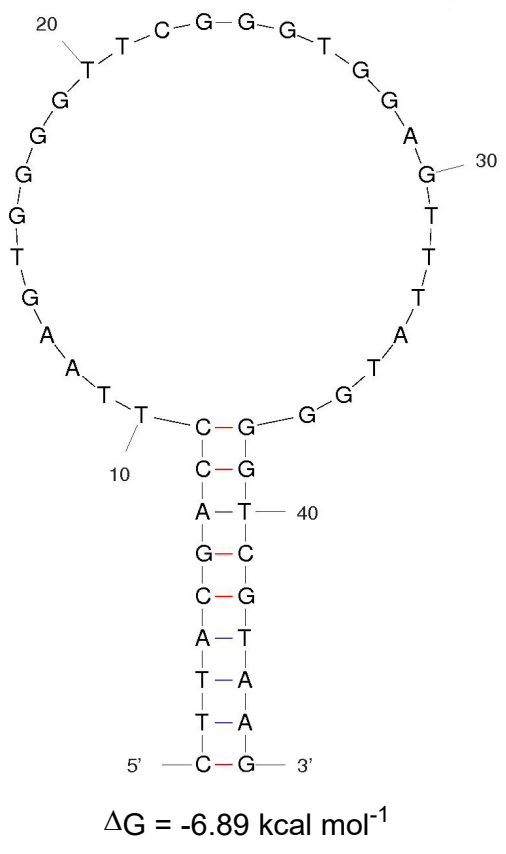

Figure 6-11. Secondary structure of SCA2.1 as predicted by Mfold at selection buffer ion concentrations $\left(20 \mathrm{mM} \mathrm{Na}^{+}\right.$and $\left.0.5 \mathrm{mM} \mathrm{Mg}^{2+}\right)$ at $23^{\circ} \mathrm{C}$. Estimated free energy of formation $(\Delta \mathrm{G})$ is also shown. 
Table 6-3. The sequences of the N30 random domain of the oligonucleotides from the final enriched pool. The prevalence of each oligonucleotide is ranked from highest to lowest based on the number of counts.

\begin{tabular}{ccc}
\hline No. & Sequences (5' - 3') & Counts (total 50) \\
\hline 1 (SCA2.1) & CTTAAGTGGGGTTCGGGTGGAGTTTATGGG & 30 \\
2 & TGAGAAGTGTGATTCAGTATGTTTTCCGAA & 7 \\
3 & CGAGAAGTGTGTTCAGTGAGTTTTCCGAGG & 4 \\
4 & CGCGGGGGTGGCTGGGGGTGTCTAGCAGAG & 2 \\
5 & ATTAAGTGGGGTTCGGGTGGAGTTTATGGG & 1 \\
6 & CCTTGGGTAGGTCAGTGTGGGGTTAGGGA & 1 \\
7 & CTTAAGTGGGGTTCGGGCGGAGTTTATGGG & 1 \\
8 & GGGAAGTGGGGTTCGGGTGGTGTTTTCCCA & 1 \\
9 & GGGATGGGGTGCTCGGTCGGGGGTTGTGAG & 1 \\
10 & GGTAAGAGTGGTTCCAGTTGAGTTTATGCC & 1 \\
11 & GGTCAGCACCTGTCGTGGTGGAGGGGTACT & 1 \\
\hline \hline
\end{tabular}

\subsubsection{HTS analysis of the selection process}

We performed a thorough investigation of the parallel-and-serial selection process using HTS. Specifically, we sequenced our three final parallel pools (butylone P5, ethylone P5, and $\alpha$-PVP P9) and two serial pools (S3 and S6) (Figure 6-12). Analysis of the parallel pools showed that the most prevalent sequence represented $0.0079 \%$ (butylone P5), 0.0012\% (ethylone P5), and 4.6\% ( $\alpha$-PVP P9) of their respective pool. The greater enrichment observed in the $\alpha$-PVP P9 pool is probably due to the additional four rounds of parallel selection. Nevertheless, no particular sequence dominated any of these parallel pools, which indicated that they were not yet highly enriched. Meanwhile, the SCA2.1 family (see methods) comprised $0.011 \%$ of the butylone P5 pool and $0.00095 \%$ of the $\alpha$-PVP P9 pool, which is notably higher than the median sequence abundance in each pool (0.00019\% for butylone P5 and $0.00032 \%$ for $\alpha$-PVP P9), showing that parallel selection enriches cross-reactive aptamers. We noted that ethylone P5 pool did not show any signs of enrichment. To determine if cross-reactive aptamers were present or lost in 
the ethylone pool, we performed an additional three rounds of parallel selection for this pool and then performed high-throughput sequencing with the resulting ethylone P8 pool. We found that the SCA2.1 family represented $0.1 \%$ of this pool, which implies that these sequences were originally present in the ethylone P5 pool, but at an amount below the detection capability of the HTS method we employed.

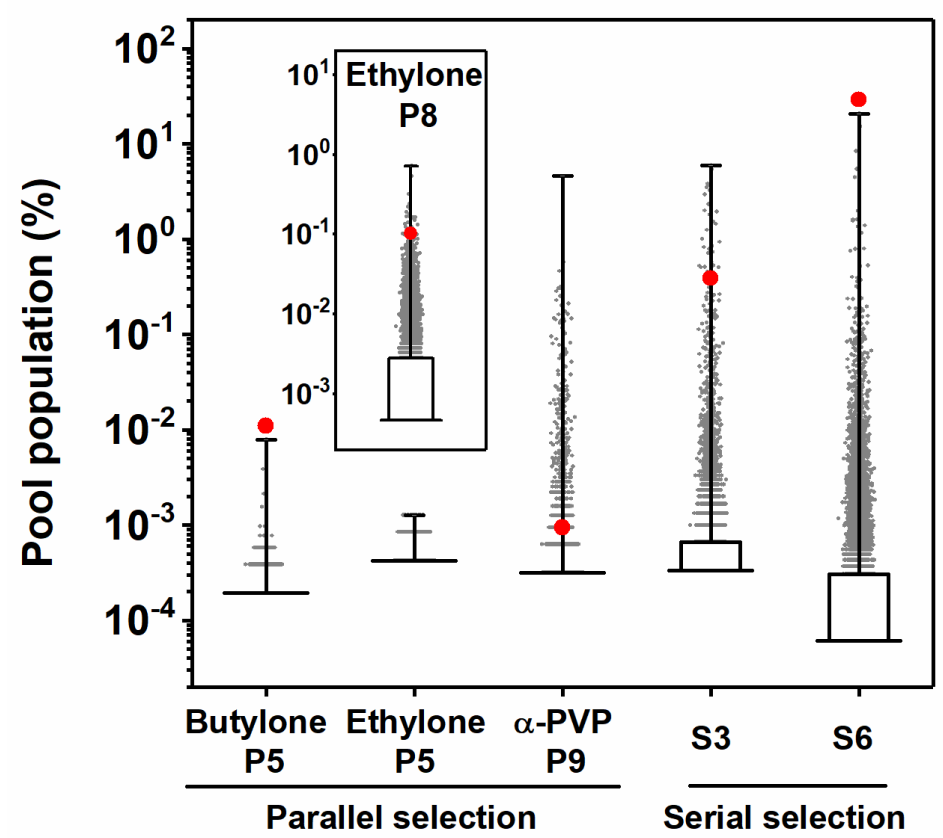

Figure 6-12. Box and whisker plots of the population distribution of sequences. Population distribution of sequences after parallel selection (butylone P5, ethylone P5, ethylone P8, and $\alpha$ PVP P9) and each round of serial selection (S3 and S6) are shown. The longest horizontal line indicates the 50th percentile, with the boundaries of the box indicating the $5^{\text {th }}$ - and $95^{\text {th }}$ percentile, and the whiskers indicate the highest and lowest values of the results. Sequences with population above $95^{\text {th }}$ percentile are plotted as grey dots. The SCA2.1 family is plotted as a red dot in each pool, except for the ethylone P5 pool where no such sequences were detected. Inset shows the pool population distribution after eight rounds of parallel selection using ethylone as target. Given the high diversity of the parallel pools, the lowest value, $5^{\text {th }}, 50^{\text {th }}$, and $95^{\text {th }}$ percentile all overlap, thus the box and lowest whisker cannot be seen. For the serial pools, the lowest value, $5^{\text {th }}$ and $50^{\text {th }}$ percentile overlap, thus the bottom portion of the box and the lowest whisker is likewise not apparent.

Upon combining the three parallel pools, the SCA2.1 family consisted of $0.0041 \%$ of the combined pool (median population: $0.000086 \%$ ) based on our sequencing data. Such enrichment allowed for exponential enrichment of cross-reactive aptamers during serial 
selection, wherein the population of the SCA2.1 family increased to $0.39 \%$ of the S3 pool and $29 \%$ of the S6 pool. Indeed, parallel selection made serial selection highly efficient. It is likely that performing serial selection without parallel selection would result in loss of cross-reactive aptamers, given the generally low copy number of such sequences in initial rounds.
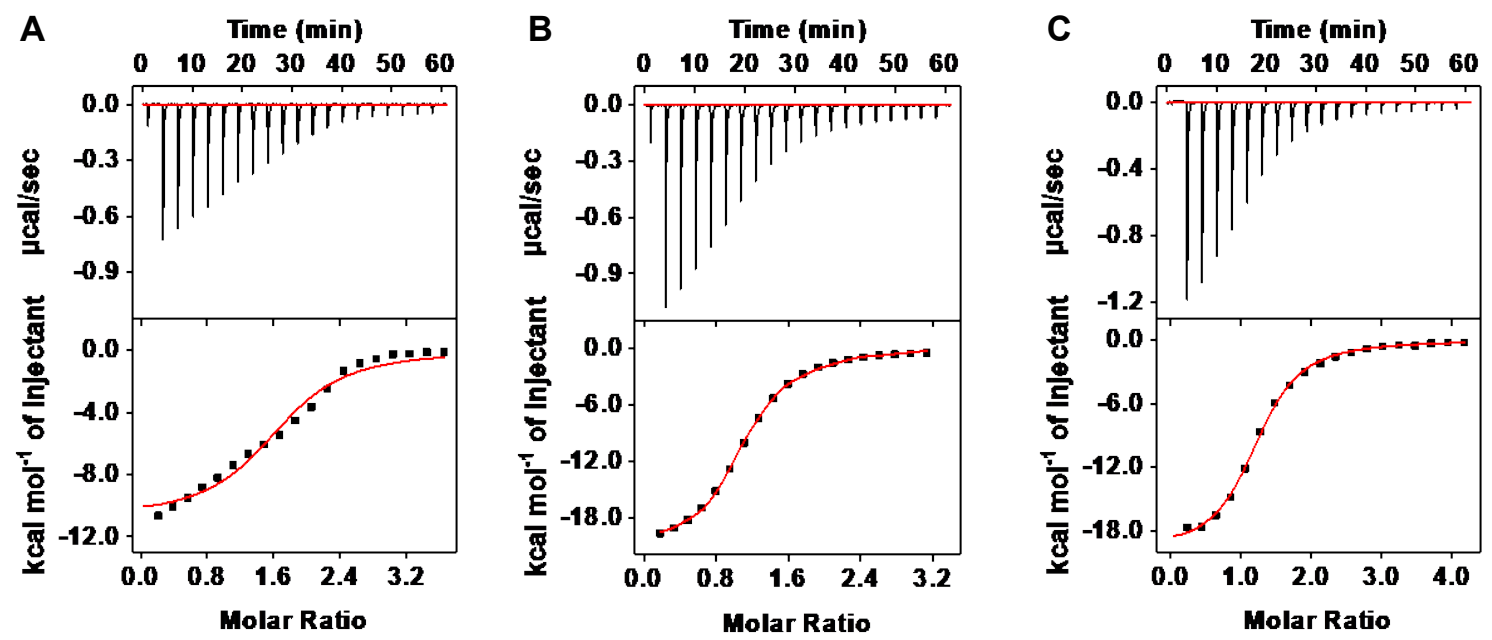

Figure 6-13. Characterization of the target-binding affinity of SCA2.1 using ITC. Top panels present raw data showing the heat generated from each titration of (A) $\alpha$-PVP, (B) ethylone and (C) butylone to SCA2.1, while bottom panels show the integrated heat of each titration after correcting for dilution heat of the titrant. ITC data were fitted using a single-site model and the binding parameters are shown in Table 6-4.

Table 6-4. Binding parameters of SCA2.1 to $\alpha$-PVP, ethylone and butylone, as characterized by fitting ITC data with a one-site or modified two-site binding model.

\begin{tabular}{ccccccc}
\hline Targets & \multicolumn{2}{c}{$\boldsymbol{\alpha}$-PVP } & \multicolumn{2}{c}{ Ethylone } & \multicolumn{2}{c}{ Butylone } \\
Binding model & One-site & Two-site & One-site & Two-site & One-site & Two-site \\
\hline $\mathrm{N}_{1}$ (target/aptamer) & $1.66 \pm 0.05$ & $1.10 \pm 0.01$ & $1.09 \pm 0.01$ & $1.06 \pm 0.06$ & $1.20 \pm 0.01$ & $1.06 \pm 0.09$ \\
$\mathbf{K}_{\mathbf{D} 1} / \boldsymbol{\mu M}$ & $2.36 \pm 0.55$ & $0.09 \pm 0.02$ & $1.62 \pm 0.06$ & $0.10 \pm 0.03$ & $1.81 \pm 0.13$ & $0.16 \pm 0.05$ \\
$\Delta \mathrm{H}_{1} / \mathrm{kcal} \mathrm{mol}^{-1}$ & $-10.8 \pm 0.5$ & $-16.5 \pm 0.1$ & $-21.5 \pm 0.1$ & $-23.1 \pm 0.2$ & $-19.9 \pm 0.3$ & $-25.9 \pm 0.6$ \\
$\mathrm{~N}_{2}$ (target/aptamer) & $0.91 \pm 0.16$ & & $0.90 \pm 0.03$ & & $0.98 \pm 0.04$ \\
$\mathbf{K}_{\mathbf{D} 2} / \boldsymbol{\mu M}$ & $18.5 \pm 7.7$ & & $2.22 \pm 0.09$ & & $1.59 \pm 0.14$ \\
$\Delta \mathrm{H}_{2} / \mathrm{kcal} \mathrm{mol}^{-1}$ & & $-12.2 \pm 4.4$ & & $-19.2 \pm 0.6$ & & $-12.9 \pm 2.7$ \\
\hline \hline
\end{tabular}




\subsubsection{Characterization of the affinity and specificity of the isolated aptamer}

We then characterized the affinity of SCA2.1 for the selection targets and specificity against interferents using isothermal titration calorimetry (ITC). We titrated a 300-400 $\mu \mathrm{M}$ solution of target into a $20 \mu \mathrm{M}$ solution of the aptamer, recorded the heat released by each titration, and integrated these data to generate a binding curve. Curve fitting with a one-site binding model resulted in atypical binding stoichiometries $(\mathrm{N})$ and less-thanoptimal fitting, especially for $\alpha$-PVP which has a non-sigmoidal curve (Figure 6-13 and

Table 6-4). Given that synthetic cathinones are chiral molecules and a racemic mixture of the targets was employed for SELEX, we hypothesized that the aptamer may have differential binding affinity for each enantiomer. The pure enantiomers of the three selection targets were not commercially available, but the high cross-reactivity of the aptamer allowed us to use enantiomers for another synthetic cathinone, (-)- and (+)MDPV, to confirm our hypothesis. ITC data indicated that the aptamer binds to one target molecule, with $\mathrm{N}=0.92$ and 0.95 for (-)- and (+)-MDPV, respectively, and exhibits 100 fold greater affinity for the (-) enantiomer $\left(\mathrm{K}_{\mathrm{D}}=46.5 \pm 7.5 \mathrm{nM}\right)$ relative to the $(+)$ enantiomer $\left(\mathrm{K}_{\mathrm{D}}=3.61 \pm 0.12 \mu \mathrm{M}\right)$ (Figure 6-14, $\mathrm{A}$ and $\mathrm{B}$, and Table 6-5). We also performed ITC by titrating racemic MDPV into a solution of SCA2.1 and observed a binding curve similar in appearance to that of $\alpha$-PVP. Using a modified two-set-of-sites model (see methods for detail) to fit the result (Figure 6-14C), we obtained similar binding parameters to those produced by the titration of each enantiomer alone (Table $6-5,( \pm)$-MDPV). This confirmed that the modified model is appropriate to describe such binding phenomenon. 

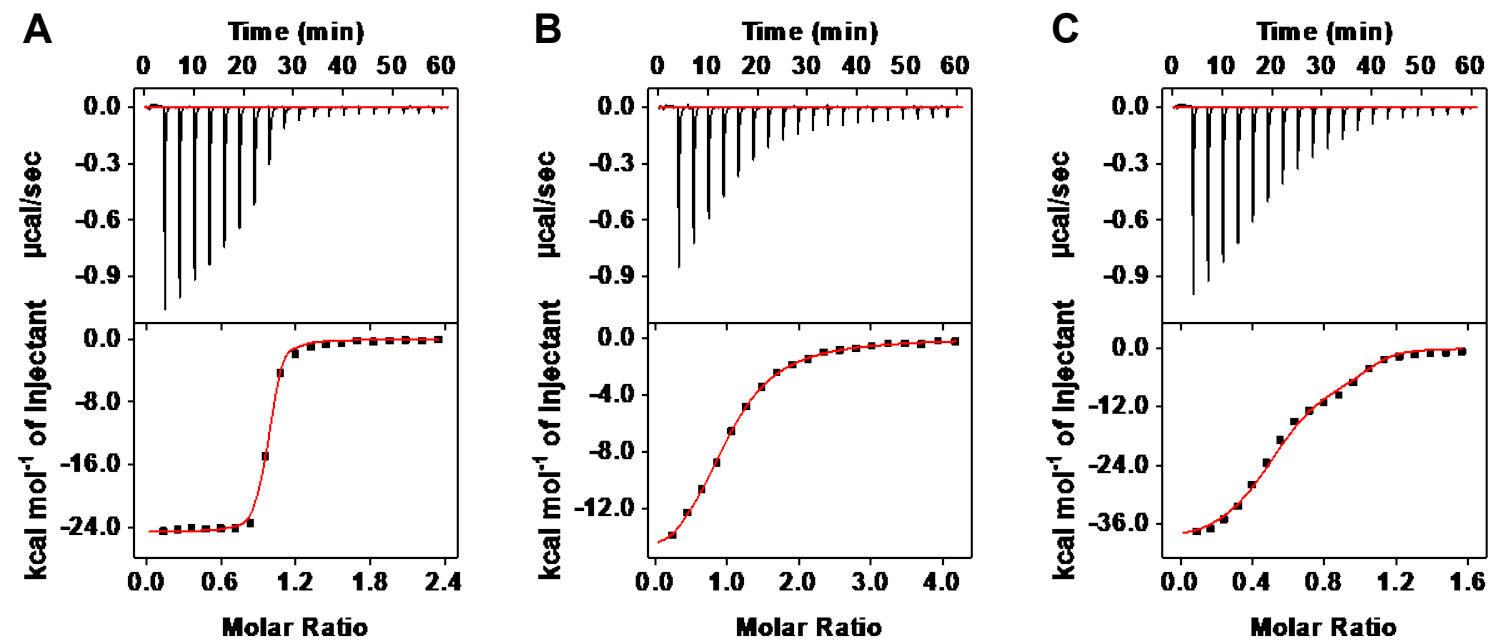

Figure 6-14. Characterization of the synthetic-cathinone-binding affinity of SCA2.1 using ITC. Top panels present raw data showing the heat generated from each titration of (A) (-)-MDPV, (B) $(+)$-MDPV and (C) ( \pm )-MDPV to SCA2.1, while bottom panels show the integrated heat of each titration after correcting for dilution heat of the titrant. ITC data obtained with (-)-MDPV and (+)MDPV were fitted using a single-site model, and ITC data obtained with $( \pm)$-MDPV were fitted with a modified two-site binding model. All binding parameters are shown in Table 6-4.

A

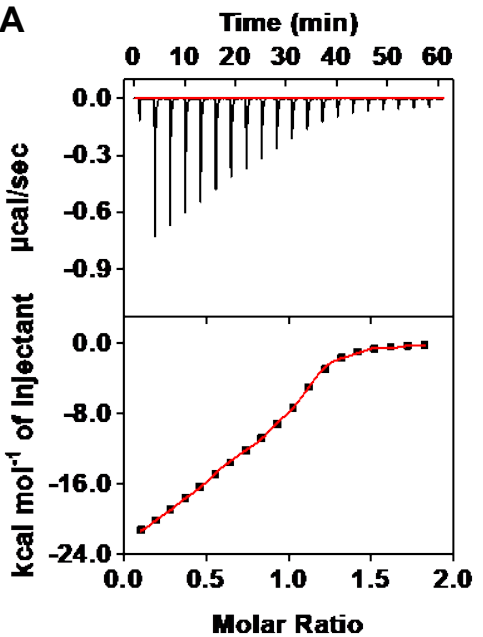

B

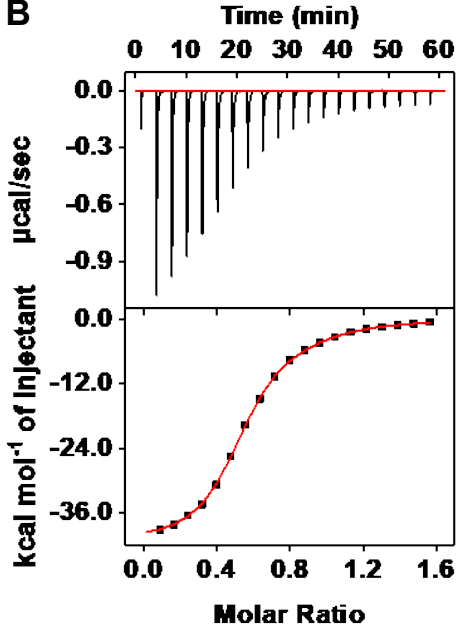

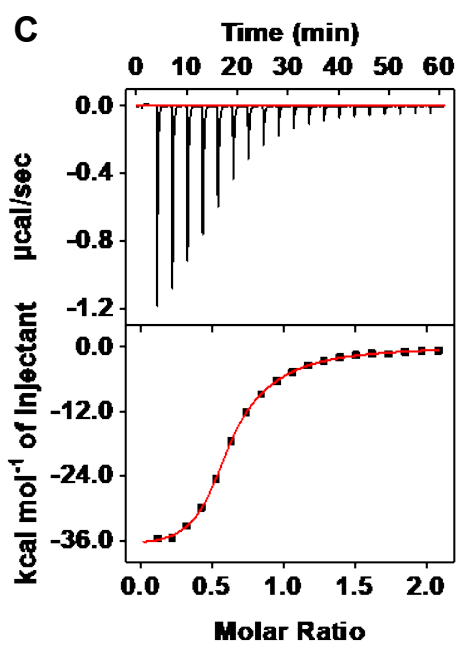

Figure 6-15. Characterization of the target-binding affinity of SCA2.1 using ITC. Top panels present raw data showing the heat generated from each titration of (A) $\alpha-P V P,(B)$ ethylone and (C) butylone to SCA2.1, while bottom panels show the integrated heat of each titration after correcting for dilution heat of the titrant. ITC data were fitted with a modified two-site binding model and the binding parameters are shown in Table 6-4. 
Table 6-5. Binding parameters of SCA2.1 to (-)-MDPV, (+)-MDPV, $( \pm)-\mathrm{MDPV}$, as characterized by fitting ITC data with a one-site (for (-)- or (+)-MDPV) or modified two-site binding model (for $( \pm)-M D P V)$.

\begin{tabular}{cccc}
\hline Binding parameters & (-)-MDPV & (+)-MDPV & ( \pm )-MDPV \\
\hline $\mathrm{N}_{1}$ (target/aptamer) & $0.92 \pm 0.01$ & & $1.04 \pm 0.06$ \\
$\mathbf{K}_{\mathbf{D} 1} / \mathbf{n M}$ & $46.5 \pm 7.5$ & & $17.5 \pm 16.4$ \\
$\Delta \mathrm{H}_{1} / \mathrm{kcal} \mathrm{mol}^{-1}$ & $-24.7 \pm 0.2$ & & $-22.3 \pm 0.4$ \\
$\mathrm{~N}_{2}$ (target $/$ aptamer) & & $0.95 \pm 0.01$ & $0.91 \pm 0.08$ \\
$\mathbf{K}_{\mathbf{D} 2} / \boldsymbol{\mu M}$ & & $3.61 \pm 0.12$ & $3.77 \pm 1.34$ \\
$\Delta \mathrm{H}_{2} / \mathrm{kcal} \mathrm{mol}^{-1}$ & & $-17.2 \pm 0.2$ & $-17.8 \pm 3.5$ \\
\hline \hline
\end{tabular}
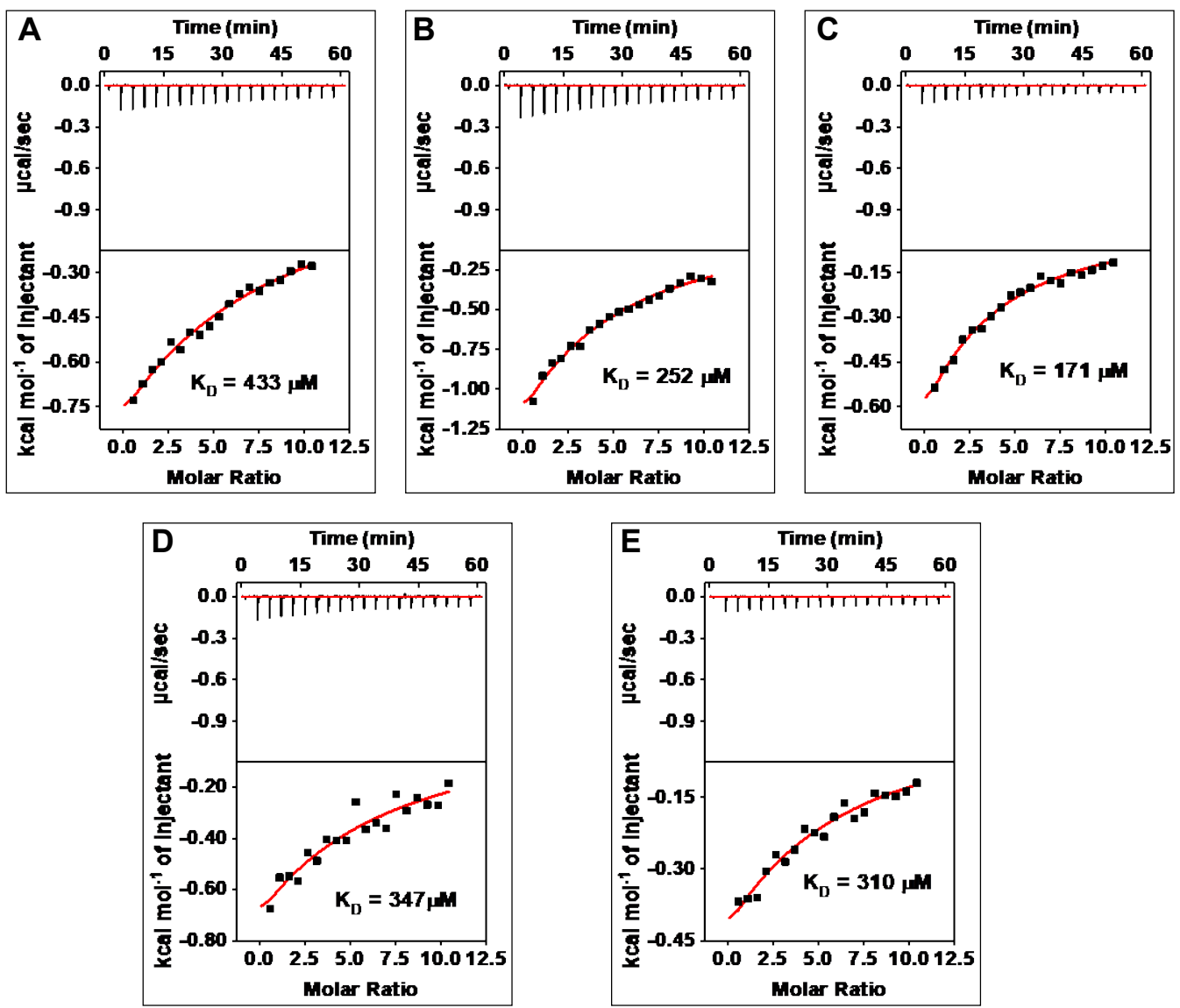

Figure 6-16. Characterization of the interferent-binding affinity of SCA 2.1 using ITC. Top panels present raw data showing the heat generated from each titration of (A) amphetamine, (B) methamphetamine, $(\mathbf{C})$ ephedrine, $(\mathbf{D})$ cocaine, and $(\mathbf{E})$ procaine to SCA2.1, while bottom panels show the integrated heat of each titration after correcting for dilution heat of the titrant. ITC data were fitted with a one-site binding model and $\mathrm{K}_{\mathrm{D}} \mathrm{S}$ are shown in the figures. 
We then used this modified two-set-of-sites model to fit the ethylone, butylone, and $\alpha$ PVP binding curves and obtained improved fitting and normal $\mathrm{N}$ values (0.9-1.1), with nanomolar affinity towards one enantiomer and micromolar affinity for the other (Figure 6-15 and Table 6-4). Opposing intuition, these results suggest that SCA2.1 can achieve not only high target-cross-reactivity, but also superior binding affinity. To determine the specificity of SCA2.1, we performed ITC with interferents that are most structurallysimilar to synthetic cathinones including amphetamine, methamphetamine, ephedrine, cocaine, and procaine. All such compounds had very low binding affinity for SCA2.1 (Figure 6-16).

\subsubsection{Evaluating the target-binding spectrum and specificity of the isolated aptamer}

We then demonstrated the analytical utility of SCA2.1 in a colorimetric dyedisplacement assay. Cy7 is a small-molecule dye that exists in equilibrium between monomer and dimer forms, which have absorbance peaks at 760 and $670 \mathrm{~nm}$, respectively. Previous studies have shown that $\mathrm{Cy} 7$ monomers can bind to hydrophobic target-binding domains of aptamers, which results in strong enhancement of absorbance at $760 \mathrm{~nm} \cdot{ }^{34,101}$ However, the binding of target to the aptamer can displace Cy7 monomer from the binding domain within seconds, which causes the dye to dimerize in aqueous solution, resulting in the reduction of absorbance at $760 \mathrm{~nm}$ and enhancement of absorbance at $670 \mathrm{~nm}$ (Figure 6-17). This approach can thus be used as a colorimetric indicator for small molecule detection. We first examined if such an assay can be employed to detect synthetic cathinones using SCA2.1. We determined the binding affinity of Cy7 to SCA2.1 by titrating different concentrations of the aptamer into a 
solution of $2 \mu \mathrm{M} \mathrm{Cy}$ (Figure 6-18A). Increasing the amount of aptamer progressively enhanced the absorbance of $\mathrm{Cy} 7$ monomer at $\sim 760 \mathrm{~nm}$, indicating binding to the aptamer. A gradual peak shift from 760 to $775 \mathrm{~nm}$ was also observed, which is consistent with previous studies ${ }^{34,101}$ showing that absorbance of the monomer can change in different microenvironments, such as when the dye binds to the aptamer. Based on Cy7 absorbance at $775 \mathrm{~nm}$, we obtained a $\mathrm{K}_{\mathrm{D}}$ of $1.6 \mu \mathrm{M}$ (Figure 6-18B). We then investigated whether the synthetic cathinone targets can efficiently displace $\mathrm{Cy} 7$ from SCA2.1. We first titrated different concentrations of butylone into a mixture of $2 \mu \mathrm{M} \mathrm{Cy} 7$ and $3 \mu \mathrm{M}$ SCA2.1, and found that increasing concentrations of butylone progressively reduced the absorbance of $\mathrm{Cy} 7$ at $775 \mathrm{~nm}$ while enhancing absorbance at $670 \mathrm{~nm}$ (Figure 6-19A). This change can be attributed to dimerization of the $\mathrm{Cy} 7$ monomer when displaced from the aptamer into solution. ${ }^{34,101}$ We used the absorbance ratio between $670 \mathrm{~nm}$ and $775 \mathrm{~nm}$ $\left(\mathrm{A}_{670} / \mathrm{A}_{775}\right)$ to calculate the signal gain and generate a calibration curve, which displayed a linear range of 0-10 $\mu \mathrm{M}$ and a measurable detection limit of $250 \mathrm{nM}$ (Figure 6-20). We obtained equivalent results with both ethylone and $\alpha$-PVP (Figure 6-19, B and C, and Figure 6-20), again confirming the high cross-reactivity of SCA2.1. Our Cy7displacement assay is also compatible with biosamples such as urine and saliva, since the absorbance range of $\mathrm{Cy} 7$ is well outside the background absorbance exhibited by these matrices. ${ }^{34}$ We obtained calibration curves with ethylone spiked into 50\% urine (Figure 6-21) and 50\% saliva (Figure 6-22) with a linear range of $0-10 \mu \mathrm{M}$ and a measurable detection limit of 80 and $120 \mathrm{nM}$, respectively. The enhanced sensitivity of the assay in these biomatrices can be possibly attributed to the higher ionic strength of the media, which may enhance target-binding to the aptamer or $\mathrm{Cy} 7$ dimerization. 


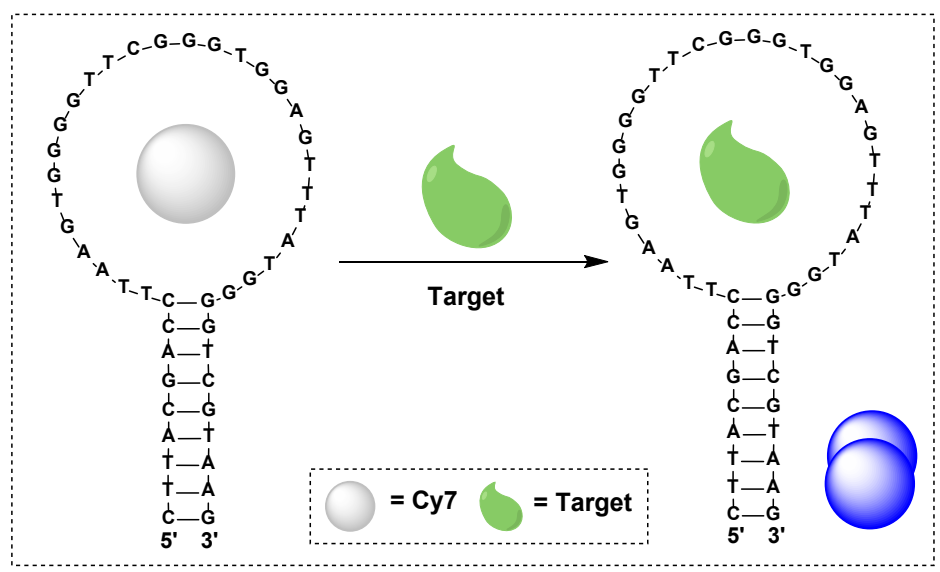

Figure 6-17. Colorimetric detection of synthetic cathinones using a Cy7-displacement assay. Schematic of the Cy7-displacement assay, wherein binding of a synthetic cathinone molecule displaces the $\mathrm{Cy} 7$ monomer from the binding domain of SCA2.1, inducing formation of $\mathrm{Cy} 7$ dimers that produce a change in the absorbance of the dye.

A

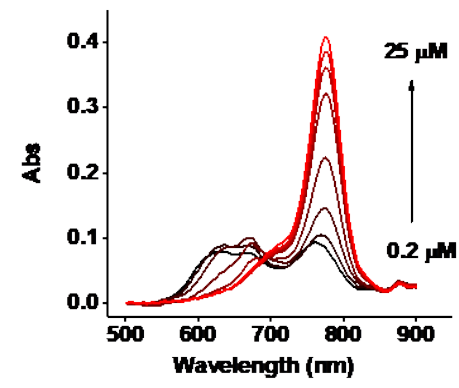

B

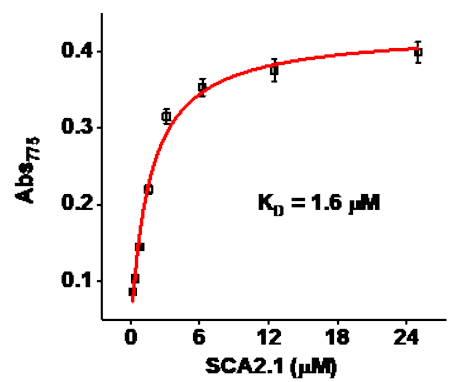

Figure 6-18. Determination of the binding affinity of $\mathrm{Cy} 7$ to SCA2.1 via a colorimetric assay. (A) Absorbance spectra of $2 \mu \mathrm{M} \mathrm{Cy} 7$ in the presence of $0.2,0.4,0.8,1.6,3.1,6.3,12.5$, or $25 \mu \mathrm{M}$ SCA2.1, with the black-to-red color gradient representing increasing concentrations. (B) Plot of the absorbance of Cy7 monomer at its peak wavelength $(775 \mathrm{~nm})$ versus concentration of SCA2.1, fitted with the Langmuir equation to determine the binding affinity of Cy7 to SCA2.1.

A

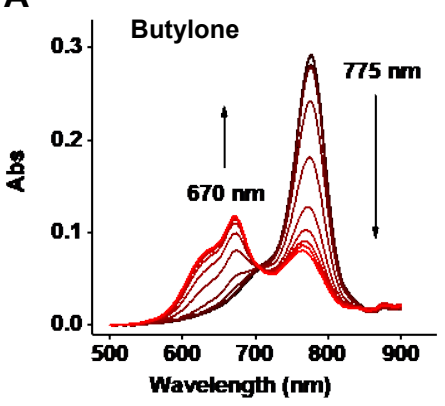

B

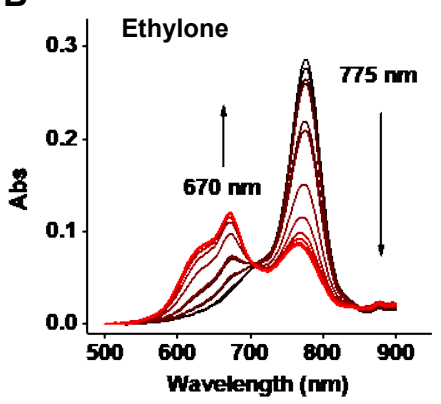

C

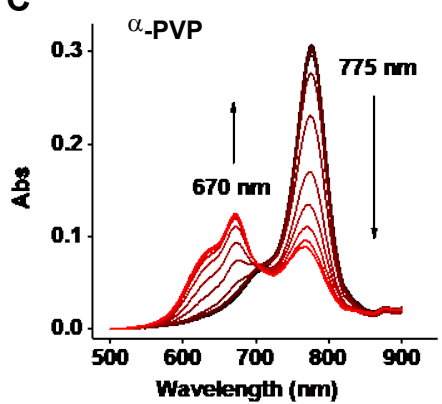

Figure 6-19. Cy7-displacement colorimetric assay for the detection of synthetic cathinones using SCA2.1. Absorbance spectra of Cy7 $(2 \mu \mathrm{M})$ in the presence of $0,0.1,0.3,0.5,1.1,2.3,4.9,10.3$, 21.6, 45.4, 95.2, or $200 \mu \mathrm{M}$ (A) butylone, (B) ethylone, (C) $\alpha$-PVP, with the black-to-red color gradient representing increasing concentrations of target. [SCA2.1] $=3 \mu \mathrm{M}$. 


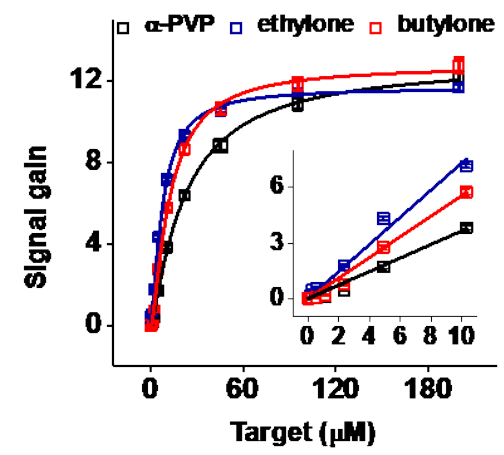

Figure 6-20. Calibration curves based on the absorbance ratio at $670 / 775 \mathrm{~nm}$ in the presence of different concentrations of $\alpha$-PVP, ethylone or butylone in buffer. The inset represents the linear range at 0 to $10 \mu \mathrm{M}$ target. Error bars show standard deviation from three measurements at each concentration. [SCA2.1] $=3 \mu \mathrm{M},[\mathrm{Cy} 7]=2 \mu \mathrm{M}$.
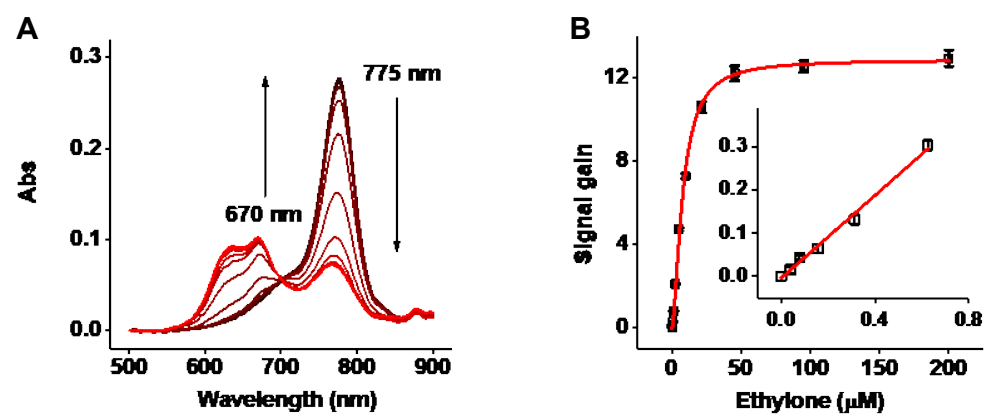

Figure 6-21. Detection of ethylone in 50\% urine using an SCA2.1-based Cy7-displacement colorimetric assay. (A) Absorbance spectra of Cy7 $(2 \mu \mathrm{M})$ in the presence of $0,0.03,0.06,0.12$, $0.25,0.53,1.11,2.33,4.90,10.28,21.60,45.35,95.24$, or $200 \mu \mathrm{M}$ ethylone, with the black-to-red color gradient representing increasing concentrations of target. (B) Assay calibration curve generated using 0-200 $\mu \mathrm{M}$ ethylone, with the inset representing the range of target concentrations from 0 to $1.1 \mu \mathrm{M}$. [SCA2.1] $=3 \mu \mathrm{M}$.
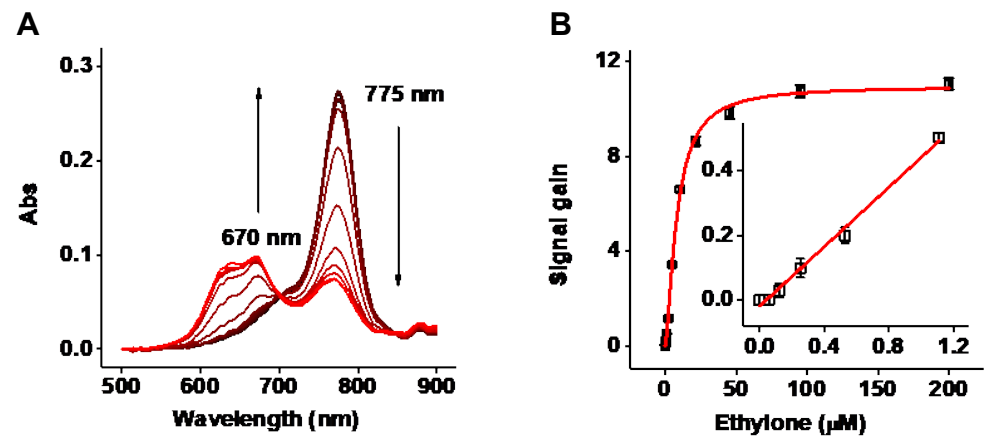

Figure 6-22. Detection of ethylone in 50\% saliva using the SCA2.1-based Cy7-displacement colorimetric assay. (A) Absorbance spectra of Cy7 $(2 \mu \mathrm{M})$ in the presence of 0, 0.03, 0.06, 0.12, $0.25,0.53,1.11,2.33,4.90,10.28,21.60,45.35,95.24$, or $200 \mu \mathrm{M}$ ethylone, with the black-to-red color gradient representing increasing concentrations of target. (B) Assay calibration curve generated using 0-200 $\mu \mathrm{M}$ ethylone, with the inset representing the range of target concentrations from 0 to $1.1 \mu \mathrm{M}$. [SCA2.1] $=3 \mu \mathrm{M}$. 
We tested the cross-reactivity of this assay for nine other synthetic cathinones, including naphyrone, MDPV, pentylone, methylone, 4-MMC, 4-FMC, 3-FMC, methcathinone and cathinone at a concentration of $50 \mu \mathrm{M}$. As expected, despite the diversity of the side chains substituents, all synthetic cathinones induced a significant change in $\mathrm{A}_{670} / \mathrm{A}_{775}$, producing a signal gain ranging from $45 \%$ to $130 \%$ relative to ethylone (Figure 6-23A). This implies that SCA2.1 mainly recognizes the $\beta$-keto phenethylamine core structure, and variations in the side chains do not significantly affect target-binding affinity. Notably, the aptamer is more cross-reactive to a broad range of synthetic cathinones than antibodies used in existing immunoassays, which achieve $>20 \%$ cross-reactivity for only five synthetic cathinones. ${ }^{236}$ SCA2.1 shows a moderate bias towards bulkier synthetic cathinones such as MDPV, naphyrone, and pentylone. Such ligands may fit better in the binding pocket compared to smaller synthetic cathinones like methcathinone or 4-FMC, achieving high binding affinity through greater interaction with the aptamer. This supports the higher signal gain observed in the assay. Importantly, our assay has excellent specificity, as the aptamer does not cross-react to non-synthetic cathinone interferents. We tested our assay with 12 different interferent compounds, including common illicit drugs (amphetamine, methamphetamine and cocaine) and cutting agents found in street samples (pseudoephedrine, ephedrine, procaine, lidocaine, benzocaine, caffeine, acetaminophen and sucrose) at a concentration of $50 \mu \mathrm{M}$. The assay yielded no response to any of these interferents (Figure 6-23A), even though many contained a partial $\beta$-keto phenethylamine structure, demonstrating that aptamer specificity can be precisely controlled through a well-designed selection approach. 
We finally determined the limit of detection for our Cy7-displacement assay for 12 synthetic cathinones and observed detection limits that varied from $40-80 \mathrm{nM}$ in $50 \%$ urine (Figure 6-24). Clearly, the high affinity of this aptamer as well as its unresponsiveness towards endogenous compounds enables sensitive screening of synthetic cathinones in biological samples. Given that the concentration of these drugs in urine typically ranges between high nanomolar to $<100 \mu \mathrm{M}$ within a few hours after consumption, ${ }^{237}$ we believe that our assay will be useful for label-free detection of synthetic cathinones in these matrices.

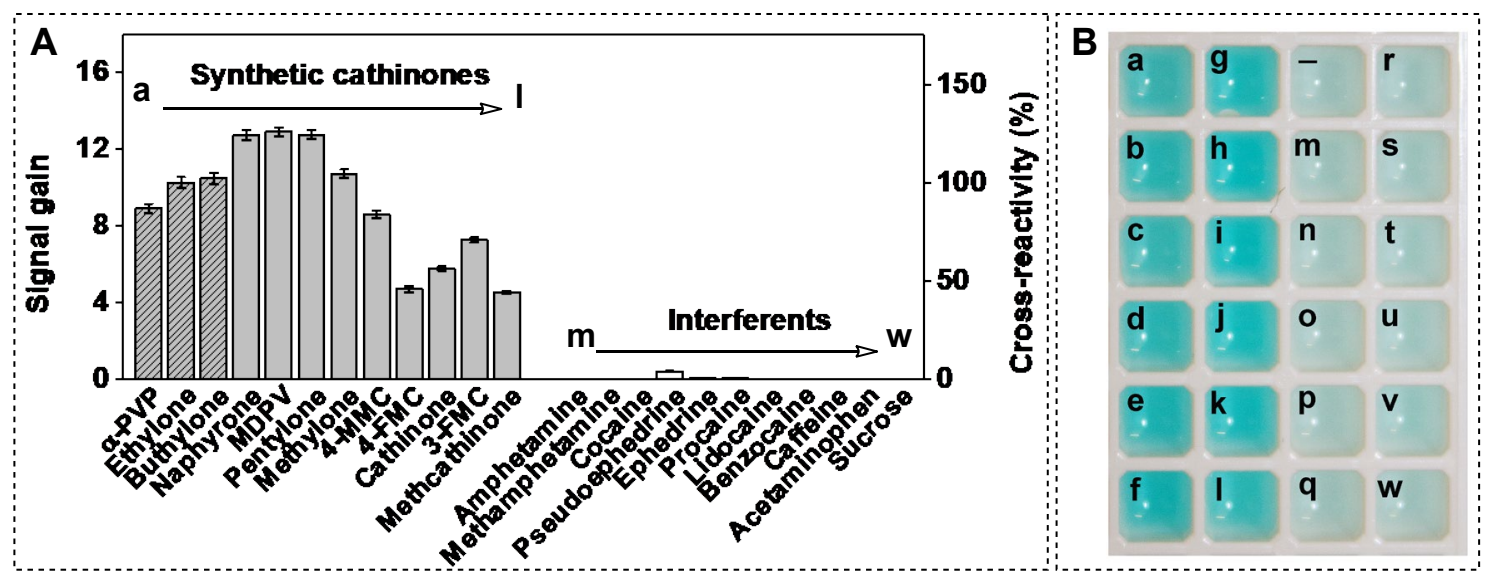

Figure 6-23. Colorimetric detection of synthetic cathinones using a Cy7-displacement assay. (A) Signal gain measured via a plate reader from the Cy7-displacement assay with 12 synthetic cathinones (gray, with the three selection targets shaded) and 11 interferents (white) at a concentration of $50 \mu \mathrm{M}$ with $3 \mu \mathrm{M}$ SCA2.1 and $2 \mu \mathrm{M}$ Cy7. Cross-reactivity is defined as the ratio of signal gain between the reference target ethylone and another synthetic cathinone or interferent multiplied by $100 \%$. Error bars show standard deviations from three measurements. (B) Nakedeye detection of synthetic cathinones using a mixture of $5 \mu \mathrm{M}$ SCA2.1 and $3.5 \mu \mathrm{M}$ Cy7. The color of the solution changes to bright blue within seconds upon addition of $50 \mu \mathrm{M}$ synthetic cathinones (a-1). However, the color appears as a faint blue color in the absence of target (-) or 50 $\mu \mathrm{M}$ of a wide range of interferents ( $\mathrm{m}-\mathrm{w})$. 

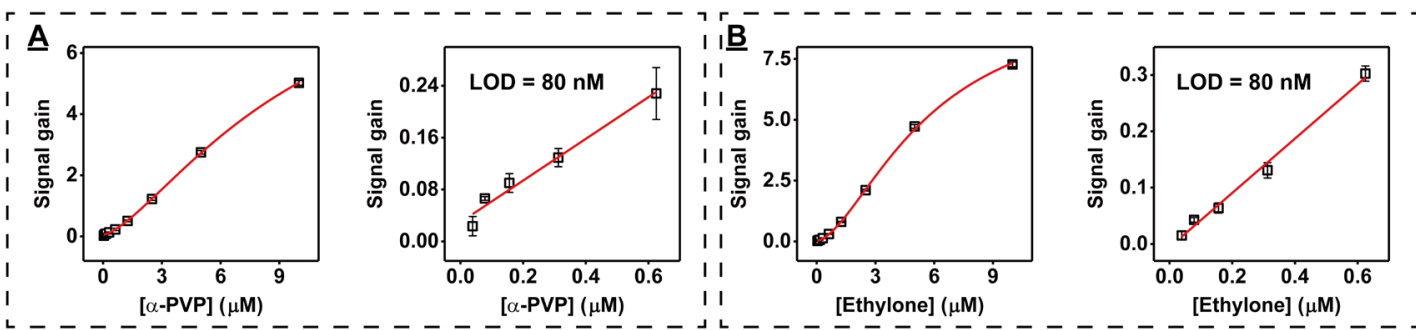

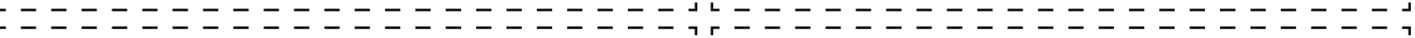
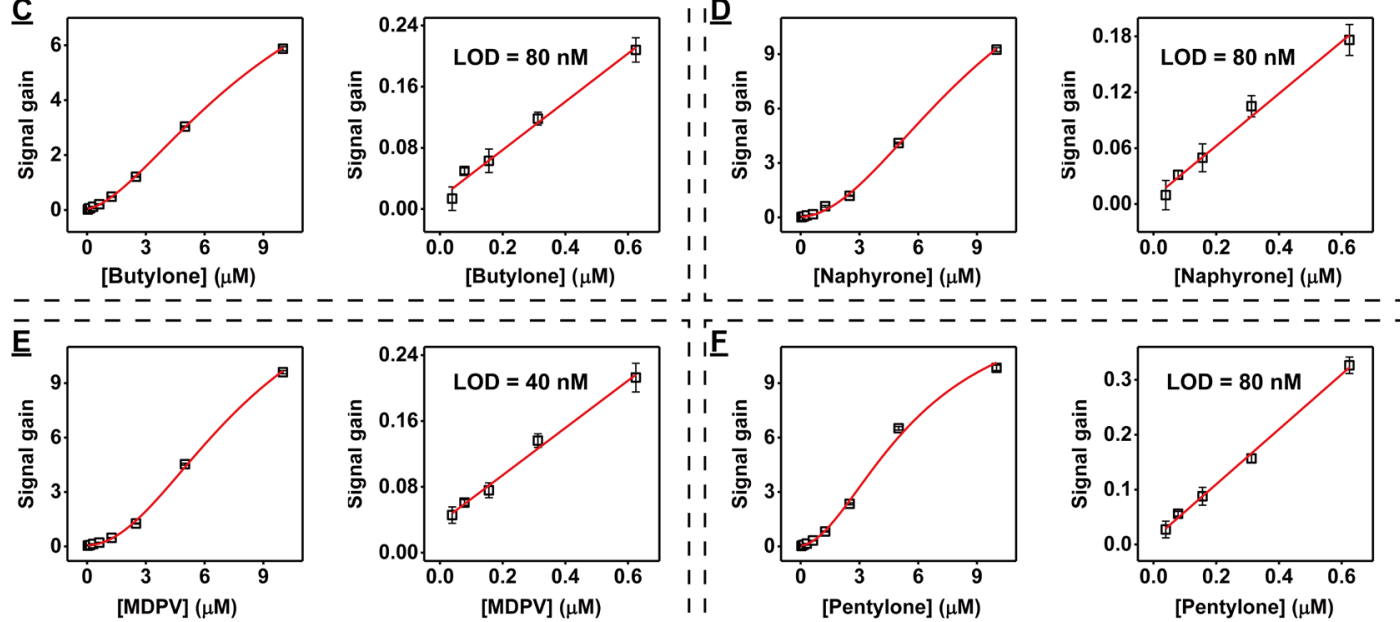

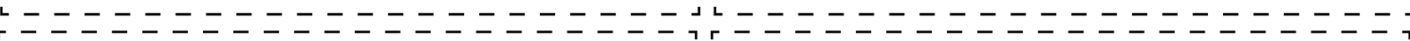
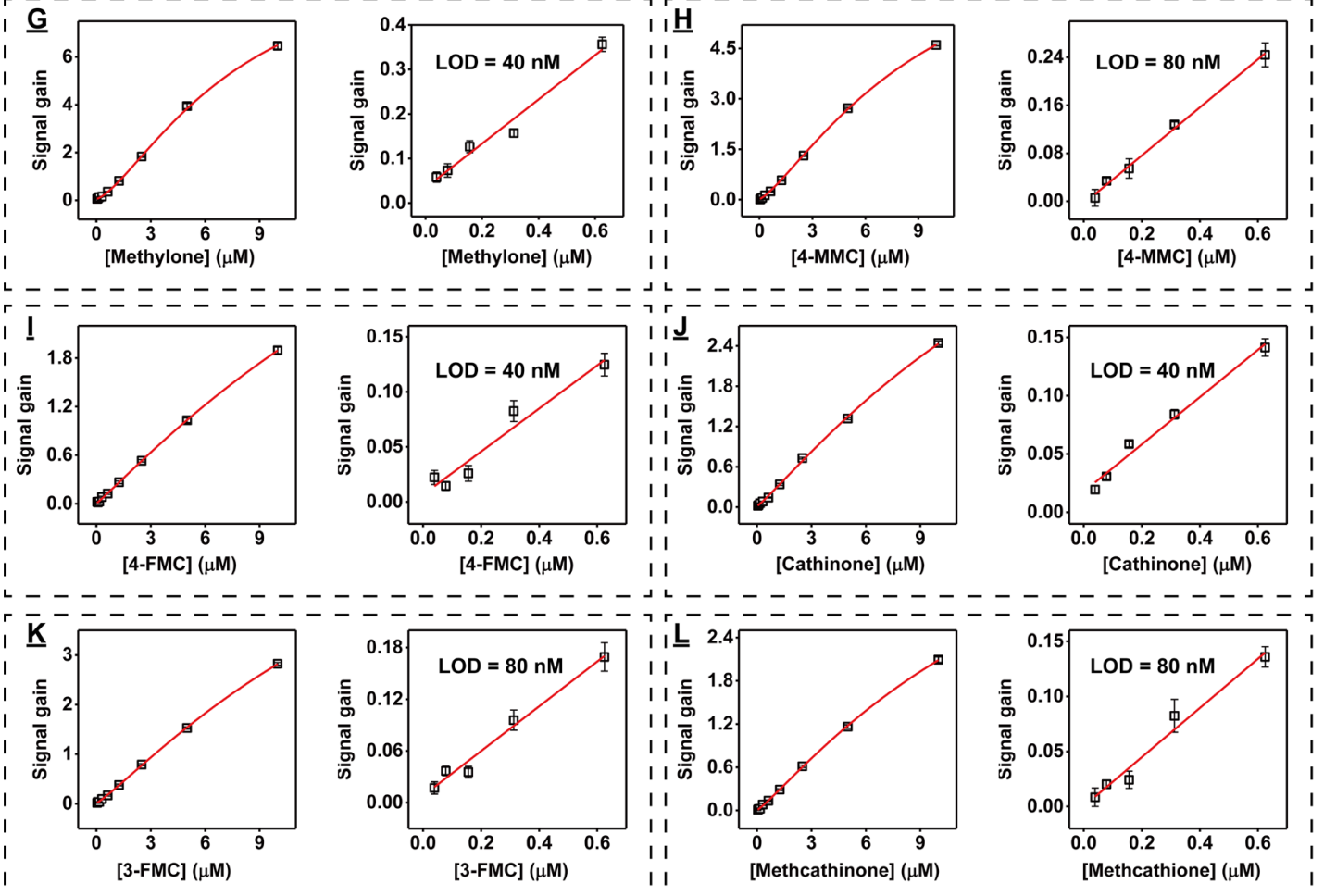

Figure 6-24. Calibration curves and linear ranges for the SCA2.1-based Cy7-displcaement assay in $50 \%$ urine based on the absorbance ratio at $670 / 775 \mathrm{~nm}$ in the presence of different concentrations of various synthetic cathinones. Error bars show standard deviation from three measurements at each concentration. [SCA2.1] $=3 \mu \mathrm{M},[\mathrm{Cy} 7]=2 \mu \mathrm{M}$. 

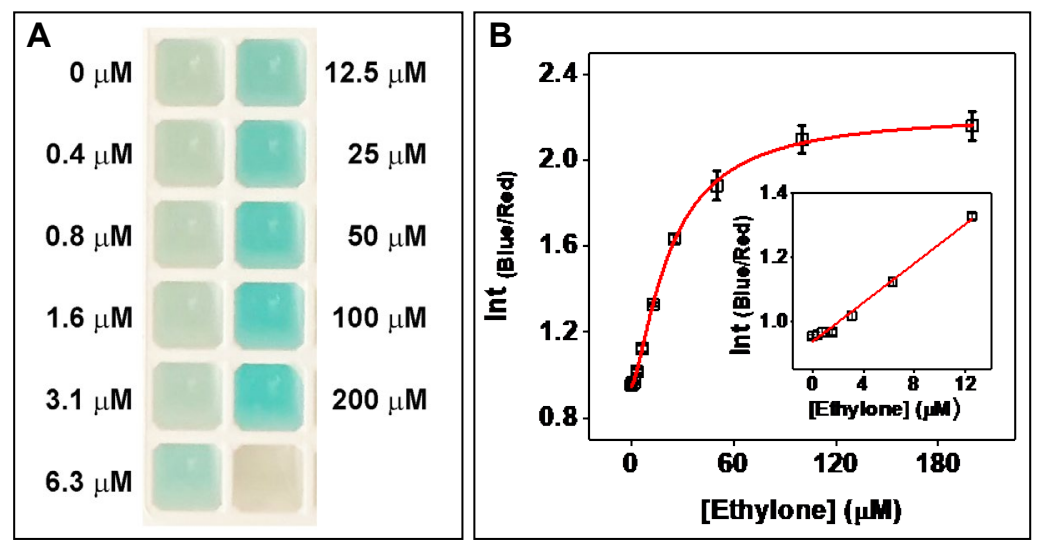

Figure 6-25. Naked-eye detection of ethylone in the concentration range of $0.4 \mu \mathrm{M}$ to $200 \mu \mathrm{M}$. (A) Photograph of the assay. The blue color change can be clearly observed at concentrations $\geq 6.3 \mu \mathrm{M}$ target. (B) A calibration curve based on the photograph was made using Image-J software. $[\mathrm{Cy} 7]=3.5 \mu \mathrm{M}$. [SCA2.1] $=5 \mu \mathrm{M}$.

We further fine-tuned our Cy7-displacement assay by using a higher concentration of the dye and aptamer in order to intensify the target-induced color change and thereby enable naked-eye detection. We challenged this assay with the aforementioned 12 synthetic cathinones and 11 interferent compounds at a concentration of $50 \mu \mathrm{M}$ with 3.5 $\mu \mathrm{M}$ Cy7 and $5 \mu \mathrm{M}$ SCA2.1. In the absence of target, the aptamer-bound Cy7 monomer has an absorption peak at $775 \mathrm{~nm}$, which is not in the visible range, and thus the sample is practically colorless. However, when $\mathrm{Cy} 7$ is displaced by the target, the resulting dimerization-associated absorption peak at $670 \mathrm{~nm}$ causes the solution to produce a bright, clearly visible blue color. We observed that all 12 synthetic cathinones immediately induced a clear-to-blue color change in the solution, while no color change was identified upon addition of any of the interferent compounds (Figure 6-23B). Using ethylone as a target, we determined that $6.3 \mu \mathrm{M}$ is the lowest concentration that can develop a color distinguishable from the blank with the naked eye (Figure 6-25). These results demonstrate the feasibility of the Cy7-displacement assay for instrument-free onsite drug screening applications. 


\subsection{Conclusions}

Class-specificity implies a degree of both receptor promiscuity towards targets within a designated molecular family and specificity against molecules outside of the family. The development of class-specific antibodies and aptamers has proven challenging due to the lack of viable methods to precisely control receptor binding profiles and specificity. In this work, we sought to develop a new aptamer isolation strategy, parallel-and-serial selection, as an effective way to select for class-specific aptamers recognizing small molecules based on a familial molecular core structure. The parallel-and-serial selection strategy increases the likelihood of isolating broadly-cross reactive aptamers by enriching oligonucleotide pools in parallel against diversely-structured members of the designated target family and then combining and challenging the pools serially with these targets to fine tune specificity towards a particular class of targets. Here, we chose three synthetic cathinones that vary at all major substituent sites of the targeted family to ensure that broadly cross-reactive aptamers are isolated. Other target triplets could yield equally cross-reactive aptamers if they are sufficiently diverse. More targets can be employed to create broader cross-reactivity, although this will increase labor and cost requirements. By supplementing our strategy with counter-SELEX, the binding spectrum can be narrowed down to the target family, thereby avoiding unwanted cross-reactivity to structurally-similar non-target molecules.

As a demonstration, we isolated a single class-specific DNA aptamer that can bind to 12 diverse synthetic cathinones. This aptamer is insensitive to variations at all substituent sites on the core structure, and even tolerates many substituents that do not appear in our selection targets. Importantly, our aptamer does not respond to 11 structurally-similar 
compounds, some of which only differ from our targets by a single atom. We subsequently demonstrated the superior class-specificity and affinity of our aptamer in a single-step, colorimetric Cy7-displacement assay, which can detect clinically-relevant concentrations of synthetic cathinones in biomatrices ${ }^{237-239}$ and presents greater targetcross-reactivity than existing antibodies. Advantageously, this assay can also achieve naked-eye detection of synthetic cathinones at concentrations at low micromolar concentrations, which is valuable for on-site screening of seized substances.

The aptamer isolated herein displays binding characteristics that confound intuition. This aptamer has great molecular promiscuity, binding to several molecules sharing a common, defined core structure. All the while, the aptamer retains the ability to discriminate against non-target molecules that are closely related in structure to members of the designated target family, even by a single atom difference in certain instances. Impressively, the aptamer also can bind to its targets with nanomolar affinity. This was not as expected, as broad cross-reactivity and high affinity is, on the surface, counterintuitive.

Our findings significantly expand the capability of aptamers as class-specific biorecognition elements and demonstrate an unprecedented level of control over aptamer binding profiles through this new parallel-and-serial selection strategy. We believe that our approach can be used to isolate class-specific aptamers for other families of small molecules for applications relevant to medical diagnostics, environmental monitoring, food safety, and forensic science. 


\section{CHAPTER 7: Summary and Future Work}

\subsection{Summary}

Aptamer-based sensors have recently gained considerable attention for diverse sensing applications such as drug detection, medical diagnostics, and environmental monitor. However, the detection of small molecules using aptamers has been challenging. This dissertation aims to remedy this problem by generating functionalized signal-reporting aptamers via several generally applicable aptamer engineering and isolation strategies.

Split aptamers that reassemble in the presence of a target ligand have excellent targetspecificity and are compatible with various sensing platforms. However, the splitting of an aptamer is typically accompanied with reduction in target-binding affinity, which mitigates any overall gains in sensitivity. In Chapter 3, we for the first time present a simple approach to greatly enhance the target-response of split aptamers utilizing a cooperative binding mechanism. We experimentally demonstrate that the resulting CBSAs exhibit higher target binding affinity and are far more responsive in terms of target-induced aptamer assembly compared to parent split aptamers containing a single binding domain. Using a fluorescence assay developed from a cocaine-binding CBSA, we were able to detect cocaine at concentrations as low as $50 \mathrm{nM}$ within 15 minutes in $10 \%$ saliva without signal amplification. This limit of detection is 200 -fold better than a similar previously reported fluorescence assay utilizing a split aptamer with a single binding domain. Given the simplicity of engineering a CBSA from a given aptamer, we were able to develop CBSA-based assays for other small molecules such as DIS and MDPV from aptamers with three-way-junction (TWJ) structured binding domains. 
In Chapter 4, we further describe a general and simple approach for the development of rapid and sensitive CBSA-based EATR-amplified sensor platforms for the detection of small-molecule targets. As a demonstration, we developed a CBSA-based EATRamplified fluorescence assay to detect DIS, a potential cancer biomarker, in urine samples. This assay had 100 -fold greater sensitivity relative to the same CBSA-based assay without EATR amplification, with a detection limit of $1 \mu \mathrm{M}$ in $50 \%$ urine and a turnaround time of 30 minutes. To further demonstrate the generality of our assay, we utilized a cocaine-binding CBSA to develop rapid instrument-free colorimetric assays that report target-binding events through EATR-mediated aggregation of CBSA-modified gold nanoparticles. We achieved naked-eye detection of low micromolar concentrations of cocaine within 20 minutes.

Most aptamer-based sensors, including CBSA-based assays, utilize structureswitching functionalized aptamers that undergo a major conformational change upon target binding. However, aptamers usually do not innately undergo target-bindinginduced conformational changes, and the development of these so-called 'structureswitching aptamers' typically entails a multi-stage process of sequence engineering and chemical modification. To simplify the process of aptamer-based sensor development, in Chapter 5, we for the first time report a simple SELEX strategy for directly isolating small-molecule-binding aptamers with intrinsic dye-displacement signal-reporting functionality. These aptamers can transduce target-binding events into a change of dye absorbance. Specifically, we first demonstrated that the small-molecule dye diethylthiatricarbocyanine (Cy7), can bind within the central cavities of DNA TWJs in a sequence-independent manner, and this interaction significantly changes the dye's 
absorbance spectra. We then designed a TWJ-structured DNA library and employed it to isolate an aptamer against MDPV, a synthetic cathinone that is an emerging drug of abuse. This aptamer intrinsically binds Cy7 within its TWJ-structured target-binding domain, but the presence of MDPV efficiently displaces the dye into solution, resulting in a significant change in absorption spectra of the dye within seconds. The Cy7displacement assay is label-free, rapid, sensitive, simple, and does not require sequence engineering or modification of the aptamer. The assay detects MDPV at concentrations as low as $300 \mathrm{nM}$ with strong cross-reactivity to other synthetic cathinone analogs, while remaining minimally cross-reactive to structurally-similar non-cathinone compounds and common cutting agents. Importantly, such dye-displacement assay can be generalized for any small-molecule target isolated from the same TWJ-structured library.

To further control the target-binding spectra of isolated aptamers, in Chapter 6, we describe a novel parallel-and-serial SELEX strategy employing multiple structurallyrelated targets for isolating class-specific DNA aptamers. Such aptamers display both familial promiscuity and specificity towards a designated molecular family and are highly desirable for various applications, in particular, for the recognition and sensing of structurally-similar small molecules. As a demonstration, we isolated an aptamer crossreactive to the synthetic cathinone family. The aptamer displays binding characteristics that confound intuition, having high molecular promiscuity and nanomolar affinity for 12 synthetic cathinones, while retaining the ability to discriminate against 11 non-target compounds that are closely related in structure, even by a single atom difference in certain instances. Leveraging the qualities of this aptamer, instantaneous colorimetric detection of synthetic cathinones at nanomolar concentrations in biological samples is 
achieved. This assay exemplifies the true potential of class-specific aptamers and generally represents a highly efficient means of screening specific small-molecule families. As a whole, this work shows that nucleic-acid-based aptamers can exhibit a combination of broad target-cross-reactivity, high affinity, and remarkable specificity. Such feat has yet to be achieved by any existing antibodies or other bioreceptors in general for small molecules.

\subsection{Future work}

This dissertation demonstrates that CBSAs are highly target-responsive and adaptable with different sensing platforms for sensitive detection of small-molecule targets. Since many other optical and electrochemical sensing strategies have been developed that employ target-induced split aptamer assembly, it should be feasible to integrate our CBSA into these platforms. For example, split G-quadruplex-structured DNAzymes with peroxidase activity have been employed as label-free, colorimetric reporters for DNA detection. Such split DNAzymes can be grafted onto CBSAs for label-free visual detection of small-molecule targets within minutes. Alternatively, CBSAs can be incorporated into the well-established electrochemical aptamer-based sensor for rapid detection of small molecules in complex matrices such as blood. In this work, we have developed a straightforward strategy to engineer CBSAs from aptamers with TWJ structures. In the future, new methods can be developed to engineer CBSAs from aptamers with other secondary structures, for example, stem-loop structured aptamers.

The dye-displacement aptamer-based assay has been demonstrated to be a rapid, sensitive and label-free method for small molecule detection. In this work, we have generalized this method to the aptamers with TWJ-structured target-binding domains. 
Inspired by this success, small-molecule dyes binding to other secondary structures such as stem-loop and G-quadruplex can be screened and employed to develop generally applicable dye-displacement assays for aptamers with such secondary structures.

Finally, our works have shown that the binding spectra of aptamers can be precisely manipulated using rationally designed aptamer isolation strategies. In the future, such selection strategies can be further refined to isolate aptamers with even higher specificity and affinity. Moreover, the multi-step washing/elution processes can be performed using an automatic syringe pump, significantly reducing the labor of SELEX and expediting aptamer isolation. 


\section{REFERENCES}

(1) Tuerk, C.; Gold, L. Systematic Evolution of Ligands by Exponential Enrichment: RNA Ligands to Bacteriophage T4 DNA Polymerase. Science 1990, 249, 505510 .

(2) Ellington, A. D.; Szostak, J. W. In Vitro Selection of RNA Molecules That Bind Specific Ligands. Nature 1990, 346, 818-822.

(3) Lee, J. F.; Hesselberth, J. R.; Meyers, L. A.; Ellington, A. D. Aptamer Database. Nucleic Acids Res. 2004, 32, D95-100.

(4) Hermann, T.; Patel, D. J. Adaptive Recognition by Nucleic Acid Aptamers. Science 2000, 287, 820-825.

(5) Dunn, M. R.; Jimenez, R. M.; Chaput, J. C. Analysis of Aptamer Discovery and Technology. Nat. Rev. Chem. 2017, 1, 76.

(6) Zhu, Q.; Liu, G.; Kai, M. DNA Aptamers in the Diagnosis and Treatment of Human Diseases. Molecules 2015, 20, 20979-20997.

(7) Adler, A.; Forster, N.; Homann, M.; Göringer, H. U. Post-SELEX Chemical Optimization of a Trypanosome-Specific RNA Aptamer. Comb. Chem. High Throughput Screen. 2008, 11, 16-23.

(8) Bock, L. C.; Griffin, L. C.; Latham, J. A.; Vermaas, E. H.; Toole, J. J. Selection of Single-Stranded DNA Molecules That Bind and Inhibit Human Thrombin. Nature 1992, 355, 564-566.

(9) Mairal, T.; Ozalp, V. C.; Lozano Sánchez, P.; Mir, M.; Katakis, I.; O’Sullivan, C. K. Aptamers: Molecular Tools for Analytical Applications. Anal. Bioanal. Chem. 2008, 390, 989-1007.

(10) Lee, J. H.; Yigit, M. V; Mazumdar, D.; Lu, Y. Molecular Diagnostic and Drug Delivery Agents Based on Aptamer-Nanomaterial Conjugates. Adv. Drug Deliv. Rev. 2010, 62, 592-605.

(11) Cho, E. J.; Lee, J. W.; Ellington, A. D. Applications of Aptamers as Sensors. Annu. Rev. Anal. Chem. 2009, 2, 241-264.

(12) Nováková, L.; Vlčková, H. A Review of Current Trends and Advances in Modern Bio-Analytical Methods: Chromatography and Sample Preparation. Anal. Chim. Acta 2009, 656, 8-35.

(13) Harper, L.; Powell, J.; Pijl, E. M. An Overview of Forensic Drug Testing Methods and Their Suitability for Harm Reduction Point-of-Care Services. Harm Reduct. J. 2017, 14, 52. 
(14) Posthuma-Trumpie, G. A.; Korf, J.; van Amerongen, A. Lateral Flow (Immuno)Assay: Its Strengths, Weaknesses, Opportunities and Threats. A Literature Survey. Anal. Bioanal. Chem. 2009, 393, 569-582.

(15) Pendergrast, P. S.; Marsh, H. N.; Grate, D.; Healy, J. M.; Stanton, M. Nucleic Acid Aptamers for Target Validation and Therapeutic Applications. J. Biomol. Tech. 2005, 16, 224-234.

(16) Toh, S. Y.; Citartan, M.; Gopinath, S. C. B.; Tang, T.-H. Aptamers as a Replacement for Antibodies in Enzyme-Linked Immunosorbent Assay. Biosens. Bioelectron. 2015, 64, 392-403.

(17) Antibodies a Laboratory Manual, 2nd ed.; Greenfield, E. A., Ed.; CSH Press: New York, 2014.

(18) Zhuo, Z.; Yu, Y.; Wang, M.; Li, J.; Zhang, Z.; Liu, J.; Wu, X.; Lu, A.; Zhang, G.; Zhang, B. Recent Advances in SELEX Technology and Aptamer Applications in Biomedicine. Int. J. Mol. Sci. 2017, 18, 2142.

(19) Rosenbaum, C. D.; Carreiro, S. P.; Babu, K. M. Here Today, Gone Tomorrow... and Back Again? A Review of Herbal Marijuana Alternatives (K2, Spice), Synthetic Cathinones (Bath Salts), Kratom, Salvia Divinorum, Methoxetamine, and Piperazines. J. Med. Toxicol. 2012, 8, 15-32.

(20) Szabó, Á.; Szendi-Szatmári, T.; Ujlaky-Nagy, L.; Rádi, I.; Vereb, G.; Szöllősi, J.; Nagy, P. The Effect of Fluorophore Conjugation on Antibody Affinity and the Photophysical Properties of Dyes. Biophys. J. 2018, 114, 688-700.

(21) Stojanovic, M. N.; de Prada, P.; Landry, D. W. Fluorescent Sensors Based on Aptamer Self-Assembly. J. Am. Chem. Soc. 2000, 122, 11547-11548.

(22) Xiao, Y.; Lubin, A. A.; Heeger, A. J.; Plaxco, K. W. Label-Free Electronic Detection of Thrombin in Blood Serum by Using an Aptamer-Based Sensor. Angew. Chem., Int. Ed. 2005, 44, 5456-5459.

(23) Nie, J.; Deng, Y.; Deng, Q. P.; Zhang, D. W.; Zhou, Y. L.; Zhang, X. X. A SelfAssemble Aptamer Fragment/Target Complex Based High-Throughput Colorimetric Aptasensor Using Enzyme Linked Aptamer Assay. Talanta 2013, 106, 309-314.

(24) Peterson, E. C.; Gunnell, M.; Che, Y.; Goforth, R. L.; Carroll, F. I.; Henry, R.; Liu, H.; Owens, S. M. Using Hapten Design to Discover Therapeutic Monoclonal Antibodies for Treating Methamphetamine Abuse. J. Pharmacol. Exp. Ther. 2007, $322,30-39$.

(25) Jenison, R. D.; Gill, S. C.; Pardi, A.; Polisky, B. High-Resolution Molecular Discrimination by RNA. Science 1994, 263, 1425-1429. 
(26) White, R.; Rusconi, C.; Scardino, E.; Wolberg, A.; Lawson, J.; Hoffman, M.; Sullenger, B. Generation of Species Cross-Reactive Aptamers Using "Toggle" SELEX. Mol. Ther. 2001, 4, 567-573.

(27) McKeague, M.; McConnell, E. M.; Cruz-Toledo, J.; Bernard, E. D.; Pach, A.; Mastronardi, E.; Zhang, X.; Beking, M.; Francis, T.; Giamberardino, A.; et al. Analysis of In Vitro Aptamer Selection Parameters. J. Mol. Evol. 2015, 81, 150161.

(28) Pobanz, K.; Lupták, A. Improving the Odds: Influence of Starting Pools on in Vitro Selection Outcomes. Methods 2016, 106, 14-20.

(29) Huizenga, D. E.; Szostak, J. W. A DNA Aptamer That Binds Adenosine and ATP. Biochemistry 1995, 34, 656-665.

(30) Wang, Y.; Rando, R. R. Specific Binding of Aminoglycoside Antibiotics to RNA. Chem. Biol. 1995, 2, 281-290.

(31) Oh, S. S.; Plakos, K.; Xiao, Y.; Eisenstein, M.; Soh, H. T. In Vitro Selection of Shape-Changing DNA Nanostructures Capable of Binding-Induced Cargo Release. ACS Nano 2013, 7, 9675-9683.

(32) Nutiu, R.; Li, Y. In Vitro Selection of Structure-Switching Signaling Aptamers. Angew. Chem., Int. Ed. 2005, 44, 1061-1065.

(33) Yang, K.-A.; Pei, R.; Stefanovic, D.; Stojanovic, M. N. Optimizing CrossReactivity with Evolutionary Search for Sensors. J. Am. Chem. Soc. 2012, 134, 1642-1647.

(34) Yu, H.; Yang, W.; Alkhamis, O.; Canoura, J.; Yang, K.-A.; Xiao, Y. In Vitro Isolation of Small-Molecule-Binding Aptamers with Intrinsic Dye-Displacement Functionality. Nucleic Acids Res. 2018, 46, e43.

(35) Kiga, D.; Futamura, Y.; Sakamoto, K.; Yokoyama, S. An RNA Aptamer to the Xanthine/Guanine Base with a Distinctive Mode of Purine Recognition. Nucleic Acids Res. 1998, 26, 1755-1760.

(36) Derbyshire, N.; White, S. J.; Bunka, D. H. J.; Song, L.; Stead, S.; Tarbin, J.; Sharman, M.; Zhou, D.; Stockley, P. G. Toggled RNA Aptamers Against Aminoglycosides Allowing Facile Detection of Antibiotics Using Gold Nanoparticle Assays. Anal. Chem. 2012, 84, 6595-6602.

(37) Stoltenburg, R.; Nikolaus, N.; Strehlitz, B. Capture-SELEX: Selection of DNA Aptamers for Aminoglycoside Antibiotics. J. Anal. Methods Chem. 2012, 415697. 
(38) Schürer, H.; Stembera, K.; Knoll, D.; Mayer, G.; Blind, M.; Förster, H. H.; Famulok, M.; Welzel, P.; Hahn, U. Aptamers That Bind to the Antibiotic Moenomycin A. Bioorg. Med. Chem. 2001, 9, 2557-2563.

(39) Niazi, J. H.; Lee, S. J.; Gu, M. B. Single-Stranded DNA Aptamers Specific for Antibiotics Tetracyclines. Bioorg. Med. Chem. 2008, 16, 7245-7253.

(40) McKeague, M.; Derosa, M. C. Challenges and Opportunities for Small Molecule Aptamer Development. J. Nucleic Acids 2012, 2012, 748913.

(41) Lou, X.; Qian, J.; Xiao, Y.; Viel, L.; Gerdon, A. E.; Lagally, E. T.; Atzberger, P.; Tarasow, T. M.; Heeger, A. J.; Soh, H. T. Micromagnetic Selection of Aptamers in Microfluidic Channels. Proc. Natl. Acad. Sci. U. S. A. 2009, 106, 2989-2994.

(42) Yang, Y.; Yang, D.; Schluesener, H. J.; Zhang, Z. Advances in SELEX and Application of Aptamers in the Central Nervous System. Biomol. Eng. 2007, 24, 583-592.

(43) Ozer, A.; Pagano, J. M.; Lis, J. T. New Technologies Provide Quantum Changes in the Scale, Speed, and Success of SELEX Methods and Aptamer Characterization. Mol. Ther. Nucleic Acids 2014, 3, e183.

(44) Spill, F.; Weinstein, Z. B.; Irani Shemirani, A.; Ho, N.; Desai, D.; Zaman, M. H. Controlling Uncertainty in Aptamer Selection. Proc. Natl. Acad. Sci. U. S. A. 2016, 113, 12076-12081.

(45) Mendonsa, S. D.; Bowser, M. T. In Vitro Evolution of Functional DNA Using Capillary Electrophoresis. J. Am. Chem. Soc. 2004, 126, $20-21$.

(46) Mendonsa, S. D.; Bowser, M. T. In Vitro Selection of High-Affinity DNA Ligands for Human IgE Using Capillary Electrophoresis. Anal. Chem. 2004, 76, 53875392.

(47) Yang, J.; Bowser, M. T. Capillary Electrophoresis-SELEX Selection of Catalytic DNA Aptamers for a Small-Molecule Porphyrin Target. Anal. Chem. 2013, 85, $1525-1530$.

(48) Wu, M.; Kempaiah, R.; Huang, P. J. J.; Maheshwari, V.; Liu, J. Adsorption and Desorption of DNA on Graphene Oxide Studied by Fluorescently Labeled Oligonucleotides. Langmuir 2011, 27, 2731-2738.

(49) Gu, H.; Duan, N.; Wu, S.; Hao, L.; Xia, Y.; Ma, X.; Wang, Z. Graphene OxideAssisted Non-Immobilized SELEX of Okdaic Acid Aptamer and the Analytical Application of Aptasensor. Sci. Rep. 2016, 6, 21665. 
(50) Nguyen, V.-T.; Kwon, Y. S.; Kim, J. H.; Gu, M. B. Multiple GO-SELEX for Efficient Screening of Flexible Aptamers. Chem. Commun. 2014, 50, 1051310516.

(51) Chen, X.; Huang, Y.; Duan, N.; Wu, S.; Xia, Y.; Ma, X.; Zhu, C.; Jiang, Y.; Wang, Z. Screening and Identification of DNA Aptamers against T-2 Toxin Assisted by Graphene Oxide. J. Agric. Food Chem. 2014, 62, 10368-10374.

(52) Yang, X.; Zhang, X.; Liu, Z.; Ma, Y.; Huang, Y.; Chen, Y. High-Efficiency Loading and Controlled Release of Doxorubicin Hydrochloride on Graphene Oxide. J. Phys. Chem. C 2008, 112, 17554-17558.

(53) Chung, S.; Moon, J.-M.; Ban, C.; Shim, Y.-B. A Simple and Fast SELEX Using an Alternating Current Potential Modulated Microfluidic Channel and an Evaluation of Sensing Ability of Aptamers. In ECS Meet. Abstr.; The Electrochemical Society, 2016; Vol. MA2016-02, p 3793.

(54) Arnold, S.; Pampalakis, G.; Kantiotou, K.; Silva, D.; Cortez, C.; Missailidis, S.; Sotiropoulou, G. One Round of SELEX for the Generation of DNA Aptamers Directed against KLK6. Biol. Chem. 2012, 393, 343-353.

(55) Berezovski, M. V; Musheev, M. U.; Drabovich, A. P.; Jitkova, J. V; Krylov, S. N. Non-SELEX: Selection of Aptamers without Intermediate Amplification of Candidate Oligonucleotides. Nat. Protoc. 2006, 1, 1359-1369.

(56) Espelund, M.; Stacy, R. A.; Jakobsen, K. S. A Simple Method for Generating Single-Stranded DNA Probes Labeled to High Activities. Nucleic Acids Res. 1990, $18,6157-6158$.

(57) Nikiforov, T. T.; Rendle, R. B.; Kotewicz, M. L.; Rogers, Y. H. The Use of Phosphorothioate Primers and Exonuclease Hydrolysis for the Preparation of Single-Stranded PCR Products and Their Detection by Solid-Phase Hybridization. PCR Methods Appl. 1994, 3, 285-291.

(58) Higuchi, R. G.; Ochman, H. Production of Single-Stranded DNA Templates by Exonuclease Digestion Following the Polymerase Chain Reaction. Nucleic Acids Res. 1989, 17, 5865.

(59) Gyllensten, U. B.; Erlich, H. A. Generation of Single-Stranded DNA by the Polymerase Chain Reaction and Its Application to Direct Sequencing of the HLADQA Locus. Proc. Natl. Acad. Sci. U. S. A. 2006, 85, 7652-7656.

(60) Svobodová, M.; Pinto, A.; Nadal, P.; O’ Sullivan, C. K. Comparison of Different Methods for Generation of Single-Stranded DNA for SELEX Processes. Anal. Bioanal. Chem. 2012, 404, 835-842. 
(61) Yang, K.-A.; Pei, R.; Stojanovic, M. N. In Vitro Selection and Amplification Protocols for Isolation of Aptameric Sensors for Small Molecules. Methods 2016, $106,58-65$.

(62) Levine, H. A.; Nilsen-Hamilton, M. A Mathematical Analysis of SELEX. Comput. Biol. Chem. 2007, 31, 11-35.

(63) Irvine, D.; Tuerk, C.; Gold, L. Selexion. J. Mol. Biol. 1991, 222, 739-761.

(64) Wang, J.; Rudzinski, J. F.; Gong, Q.; Soh, H. T.; Atzberger, P. J. Influence of Target Concentration and Background Binding on In Vitro Selection of Affinity Reagents. PLoS One 2012, 7, e43940.

(65) Ruscito, A.; DeRosa, M. C. Small-Molecule Binding Aptamers: Selection Strategies, Characterization, and Applications. Front. Chem. 2016, 4, 14.

(66) Ellington, A. D.; Szostak, J. W. Selection in Vitro of Single-Stranded DNA Molecules That Fold into Specific Ligand-Binding Structures. Nature 1992, 355, $850-852$.

(67) Verma, N.; Bhardwaj, A. Biosensor Technology for Pesticides-A Review. Appl. Biochem. Biotechnol. 2015, 175, 3093-3119.

(68) Huet, A. C.; Delahaut, P.; Fodey, T.; Haughey, S. A.; Elliott, C.; Weigel, S. Advances in Biosensor-Based Analysis for Antimicrobial Residues in Foods. TrAC, Trends Anal. Chem. 2010, 29, 1281-1294.

(69) Gandhi, S.; Suman, P.; Kumar, A.; Sharma, P.; Capalash, N.; Suri, C. R. Recent Advances in Immunosensor for Narcotic Drug Detection. BioImpacts 2015, 5, 207-213.

(70) Bianchini, M.; Radrizzani, M.; Brocardo, M. G.; Reyes, G. B.; Gonzalez Solveyra, C.; Santa-Coloma, T. A. Specific Oligobodies against ERK-2 That Recognize Both the Native and the Denatured State of the Protein. J. Immunol. Methods 2001, 252, 191-197.

(71) Song, M. Y.; Nguyen, D.; Hong, S. W.; Kim, B. C. Broadly Reactive Aptamers Targeting Bacteria Belonging to Different Genera Using a Sequential Toggle CellSELEX. Sci. Rep. 2017, 7, 43641.

(72) Powell Gray, B.; Kelly, L.; Ahrens, D. P.; Barry, A. P.; Kratschmer, C.; Levy, M.; Sullenger, B. A. Tunable Cytotoxic Aptamer-Drug Conjugates for the Treatment of Prostate Cancer. Proc. Natl. Acad. Sci. U. S. A. 2018.

(73) Reinemann, C.; Freiin von Fritsch, U.; Rudolph, S.; Strehlitz, B. Generation and Characterization of Quinolone-Specific DNA Aptamers Suitable for Water Monitoring. Biosens. Bioelectron. 2016, 77, 1039-1047. 
(74) Yang, W.; Yu, H.; Alkhamis, O.; Liu, Y.; Canoura, J.; Fu, F.; Xiao, Y. In Vitro Isolation of Class-Specific Oligonucleotide-Based Small-Molecule Receptors. Nucleic Acids Res. 2019, In Press.

(75) Pfeiffer, F.; Mayer, G. Selection and Biosensor Application of Aptamers for Small Molecules. Front. Chem. 2016, 4, 25.

(76) Song, S.; Wang, L.; Li, J.; Fan, C.; Zhao, J. Aptamer-Based Biosensors. TrAC, Trends Anal. Chem. 2008, 27, 108-117.

(77) Iliuk, A. B.; Hu, L. H.; Tao, W. A. Aptamer in Bioanalytical Applications. Anal. Chem. 2011, 83, 4440-4452.

(78) Stojanovic, M. N.; de Prada, P.; Landry, D. W. Aptamer-Based Folding Fluorescent Sensor for Cocaine. J. Am. Chem. Soc. 2001, 123, 4928-4931.

(79) Arroyo-Currás, N.; Somerson, J.; Vieira, P. A.; Ploense, K. L.; Kippin, T. E.; Plaxco, K. W. Real-Time Measurement of Small Molecules Directly in Awake, Ambulatory Animals. Proc. Natl. Acad. Sci. U. S. A. 2017, 114, 645-650.

(80) Han, K.; Liang, Z.; Zhou, N. Design Strategies for Aptamer-Based Biosensors. Sensors 2010, 10, 4541-4557.

(81) Zadeh, J. N.; Steenberg, C. D.; Bois, J. S.; Wolfe, B. R.; Pierce, M. B.; Khan, A. R.; Dirks, R. M.; Pierce, N. A. NUPACK: Analysis and Design of Nucleic Acid Systems. J. Comput. Chem. 2011, 32, 170-173.

(82) Zuker, M. Mfold Web Server for Nucleic Acid Folding and Hybridization Prediction. Nucleic Acids Res. 2003, 31, 3406-3415.

(83) Gao, S.; Zheng, X.; Jiao, B.; Wang, L. Post-SELEX Optimization of Aptamers. Anal. Bioanal. Chem. 2016, 408, 4567-4573.

(84) Fischer, N. O.; Tok, J. B.; Tarasow, T. M. Massively Parallel Interrogation of Aptamer Sequence, Structure and Function. PLoS One 2008, 3, e2720.

(85) Shangguan, D.; Tang, Z.; Mallikaratchy, P.; Xiao, Z.; Tan, W. Optimization and Modifications of Aptamers Selected from Live Cancer Cell Lines. ChemBioChem 2007, 8, 603-606.

(86) Neves, M. A.; Reinstein, O.; Saad, M.; Johnson, P. E. Defining the Secondary Structural Requirements of a Cocaine-Binding Aptamer by a Thermodynamic and Mutation Study. Biophys. Chem. 2010, 153, 9-16.

(87) Neves, M. A. D.; Reinstein, O.; Johnson, P. E. Defining a Stem Length-Dependent Binding Mechanism for the Cocaine-Binding Aptamer. A Combined NMR and Calorimetry Study. Biochemistry 2010, 49, 8478-8487. 
(88) Wang, Z.; Yu, H.; Canoura, J.; Liu, Y.; Alkhamis, O.; Fu, F.; Xiao, Y. Introducing Structure-Switching Functionality into Small-Molecule-Binding Aptamers via Nuclease-Directed Truncation. Nucleic Acids Res. 2018, 46, e81.

(89) Baker, B. R.; Lai, R. Y.; Wood, M. S.; Doctor, E. H.; Heeger, A. J.; Plaxco, K. W. An Electronic, Aptamer-Based Small-Molecule Sensor for the Rapid, Label-Free Detection of Cocaine in Adulterated Samples and Biological Fluids. J. Am. Chem. Soc. 2006, 128, 3138-3139.

(90) Zou, R. X.; Lou, X. H.; Ou, H. C.; Zhang, Y.; Wang, W. J.; Yuan, M.; Guan, M.; Luo, Z. F.; Liu, Y. Highly Specific Triple-Fragment Aptamer for Optical Detection of Cocaine. $R S C A d v$. 2012, 2, 4636-4638.

(91) Chen, A.; Yan, M.; Yang, S. Split Aptamers and Their Applications in Sandwich Aptasensors. TrAC, Trends Anal. Chem. 2016, 80, 581-593.

(92) Kent, A. D.; Spiropulos, N. G.; Heemstra, J. M. General Approach for Engineering Small-Molecule-Binding DNA Split Aptamers. Anal. Chem. 2013, 85, 9916-9923.

(93) Sharma, A. K.; Heemstra, J. M. Small-Molecule-Dependent Split Aptamer Ligation. J. Am. Chem. Soc. 2011, 133, 12426-12429.

(94) Sharma, A. K.; Kent, A. D.; Heemstra, J. M. Enzyme-Linked Small-Molecule Detection Using Split Aptamer Ligation. Anal. Chem. 2012, 84, 6104-6109.

(95) Yu, H.; Canoura, J.; Guntupalli, B.; Lou, X.; Xiao, Y. A Cooperative-Binding Split Aptamer Assay for Rapid, Specific and Ultra-Sensitive Fluorescence Detection of Cocaine in Saliva. Chem. Sci. 2017, 8, 131-141.

(96) Yu, H.; Canoura, J.; Guntupalli, B.; Alkhamis, O.; Xiao, Y. Sensitive Detection of Small-Molecule Targets Using Cooperative Binding Split Aptamers and EnzymeAssisted Target Recycling. Anal. Chem. 2018, 90, 1748-1758.

(97) Nutiu, R.; Li, Y. F. Structure-Switching Signaling Aptamers. J. Am. Chem. Soc. 2003, 125, 4771-4778.

(98) Rajendran, M.; Ellington, A. D. In Vitro Selection of Molecular Beacons. Nucleic Acids Res. 2003, 31, 5700-5713.

(99) Li, N.; Ho, C. Aptamer-Based Optical Probes with Separated Molecular Recognition and Signal Transduction Modules. J. Am. Chem. Soc. 2008, 130, 2380-2381.

(100) Yang, K. A.; Barbu, M.; Halim, M.; Pallavi, P.; Kim, B.; Kolpashchikov, D. M.; Pecic, S.; Taylor, S.; Worgall, T. S.; Stojanovic, M. N. Recognition and Sensing of Low-Epitope Targets via Ternary Complexes with Oligonucleotides and Synthetic Receptors. Nat. Chem. 2014, 6, 1003-1008. 
(101) Stojanovic, M. N.; Landry, D. W. Aptamer-Based Colorimetric Probe for Cocaine. J. Am. Chem. Soc. 2002, 124, 9678-9679.

(102) Roncancio, D.; Yu, H. X.; Xu, X. W.; Wu, S.; Liu, R.; Debord, J.; Lou, X. H.; Xiao, Y. Y. A Label-Free Aptamer-Fluorophore Assembly for Rapid and Specific Detection of Cocaine in Biofluids. Anal. Chem. 2014, 86, 11100-11106.

(103) Ji, D. Y.; Wang, H. Q.; Ge, J.; Zhang, L.; Li, J. J.; Bai, D. M.; Chen, J.; Li, Z. H. Label-Free and Rapid Detection of ATP Based on Structure Switching of Aptamers. Anal. Biochem. 2017, 526, 22-28.

(104) Pei, R.; Stojanovic, M. N. Study of Thiazole Orange in Aptamer-Based DyeDisplacement Assays. Anal. Bioanal. Chem. 2008, 390, 1093-1099.

(105) Syu, Y.-C.; Hsu, W.-E.; Lin, C.-T. Review-Field-Effect Transistor Biosensing: Devices and Clinical Applications. ECS J. Solid State Sci. Technol. 2018, 7, Q3196-3207.

(106) Shoorideh, K.; Chui, C. O. On the Origin of Enhanced Sensitivity in Nanoscale FET-Based Biosensors. Proc. Natl. Acad. Sci. U. S. A. 2014, 111, 5111-5116.

(107) Kim, J.; Rim, Y. S.; Chen, H.; Cao, H. H.; Nakatsuka, N.; Hinton, H. L.; Zhao, C.; Andrews, A. M.; Yang, Y.; Weiss, P. S. Fabrication of High-Performance Ultrathin In2O3 Film Field-Effect Transistors and Biosensors Using Chemical Lift-off Lithography. ACS Nano 2015, 9, 4572-4582.

(108) Weiss, P. S.; Trevor, P. L.; Cardillo, M. J. Gas-Surface Interactions on InP Monitored by Changes in Substrate Electronic Properties. J. Chem. Phys. 1989, 90, $5146-5153$.

(109) Vacic, A.; Criscione, J. M.; Rajan, N. K.; Stern, E.; Fahmy, T. M.; Reed, M. A. Determination of Molecular Configuration by Debye Length Modulation. J. Am. Chem. Soc. 2011, 133, 13886-13889.

(110) Nakatsuka, N.; Yang, K.-A.; Abendroth, J. M.; Cheung, K. M.; Xu, X.; Yang, H.; Zhao, C.; Zhu, B.; Rim, Y. S.; Yang, Y.; et al. Aptamer-Field-Effect Transistors Overcome Debye Length Limitations for Small-Molecule Sensing. Science 2018, 362, 319-324.

(111) Canoura, J.; Wang, Z.; Yu, H.; Alkhamis, O.; Fu, F.; Xiao, Y. No StructureSwitching Required: A Generalizable Exonuclease-Mediated Aptamer-Based Assay for Small-Molecule Detection. J. Am. Chem. Soc. 2018, 140, 9961-9971.

(112) Liu, J.; Cao, Z.; Lu, Y. Functional Nucleic Acid Sensors. Chem. Rev. 2009, 109, 1948-1998. 
(113) Turkevich, J.; Stevenson, P. C.; Hillier, J. A Study of the Nucleation and Growth Processes in the Synthesis of Colloidal Gold. Discuss. Faraday Soc. 1951, 11, 5575.

(114) Brust, M.; Walker, M.; Bethell, D.; Schiffrin, D. J.; Whyman, R. Synthesis of Thiol-Derivatised Gold Nanoparticles in a Two-Phase Liquid-Liquid System. $J$. Chem. Soc. Chem. Commun. 1994, No. 7, 801-802.

(115) Busbee, B. D.; Obare, S. O.; Murphy, C. J. An Improved Synthesis of HighAspect-Ratio Gold Nanorods. Adv. Mater. 2003, 15, 414-416.

(116) Senthil Kumar, P.; Pastoriza-Santos, I.; Rodríguez-González, B.; Javier García de Abajo, F.; Liz-Marzán, L. M. High-Yield Synthesis and Optical Response of Gold Nanostars. Nanotechnology 2008, 19, 015606.

(117) Millstone, J. E.; Park, S.; Shuford, K. L.; Qin, L.; Schatz, G. C.; Mirkin, C. A. Observation of a Quadrupole Plasmon Mode for a Colloidal Solution of Gold Nanoprisms. J. Am. Chem. Soc. 2005, 127, 5312-5313.

(118) Khlebtsov, N. G. Determination of Size and Concentration of Gold Nanoparticles from Extinction Spectra. Anal. Chem. 2008, 80, 6620-6625.

(119) Amendola, V.; Meneghetti, M. Size Evaluation of Gold Nanoparticles by UV-Vis Spectroscopy. J. Phys. Chem. C 2009, 113, 4277-4285.

(120) Jans, H.; Liu, X.; Austin, L.; Maes, G.; Huo, Q. Dynamic Light Scattering as a Powerful Tool for Gold Nanoparticle Bioconjugation and Biomolecular Binding Studies. Anal. Chem. 2009, 81, 9425-9432.

(121) Cutler, J. I.; Auyeung, E.; Mirkin, C. A. Spherical Nucleic Acids. J. Am. Chem. Soc. 2012, 134, 1376-1391.

(122) Rosi, N. L.; Mirkin, C. A. Nanostructures in Biodiagnostics. Chem. Rev. 2005, $105,1547-1562$.

(123) Yao, G.; Pei, H.; Li, J.; Zhao, Y.; Zhu, D.; Zhang, Y.; Lin, Y.; Huang, Q.; Fan, C. Clicking DNA to Gold Nanoparticles: Poly-Adenine-Mediated Formation of Monovalent DNA-Gold Nanoparticle Conjugates with Nearly Quantitative Yield. NPG Asia Mater. 2015, 7, e159.

(124) Wang, Z.; Zhang, J.; Ekman, J. M.; Kenis, P. J. A.; Lu, Y. DNA-Mediated Control of Metal Nanoparticle Shape: One-Pot Synthesis and Cellular Uptake of Highly Stable and Functional Gold Nanoflowers. Nano Lett. 2010, 10, 1886-1891.

(125) Mirkin, C. A.; Letsinger, R. L.; Mucic, R. C.; Storhoff, J. J. A DNA-Based Method for Rationally Assembling Nanoparticles into Macroscopic Materials. Nature 1996, 382, 607-609. 
(126) Li, Z.; Jin, R.; Mirkin, C. A.; Letsinger, R. L. Multiple Thiol-Anchor Capped DNA-Gold Nanoparticle Conjugates. Nucleic Acids Res. 2002, 30, 1558-1562.

(127) Alivisatos, A. P.; Johnsson, K. P.; Peng, X.; Wilson, T. E.; Loweth, C. J.; Bruchez, M. P.; Schultz, P. G. Organization of "nanocrystal Molecules" Using DNA. Nature 1996, 382, 609-611.

(128) Hurst, S. J.; Lytton-Jean, A. K. R.; Mirkin, C. A. Maximizing DNA Loading on a Range of Gold Nanoparticle Sizes. Anal. Chem. 2006, 78, 8313-8318.

(129) Nicewarner Peña, S. R.; Raina, S.; Goodrich, G. P.; Fedoroff, N. V.; Keating, C. D. Hybridization and Enzymatic Extension of $\mathrm{Au}$ Nanoparticle-Bound Oligonucleotides. J. Am. Chem. Soc. 2002, 124, 7314-7323.

(130) Herne, T. M.; Tarlov, M. J. Characterization of DNA Probes Immobilized on Gold Surfaces. J. Am. Chem. Soc. 1997, 119, 8916-8920.

(131) Lytton-Jean, A. K. R.; Mirkin, C. A. A Thermodynamic Investigation into the Binding Properties of DNA Functionalized Gold Nanoparticle Probes and Molecular Fluorophore Probes. J. Am. Chem. Soc. 2005, 127, 12754-12755.

(132) Wang, Z.; Kanaras, A. G.; Bates, A. D.; Cosstick, R.; Brust, M. Enzymatic DNA Processing on Gold Nanoparticles. J. Mater. Chem. 2004, 14, 578-580.

(133) Seferos, D. S.; Prigodich, A. E.; Giljohann, D. A.; Patel, P. C.; Mirkin, C. A. Polyvalent DNA Nanoparticle Conjugates Stabilize Nucleic Acids. Nano Lett. 2009, 9, 308-311.

(134) Qin, W. J.; Yung, L. Y. L. Efficient Manipulation of Nanoparticle-Bound DNA via Restriction Endonuclease. Biomacromolecules 2006, 7, 3047-3051.

(135) Prigodich, A. E.; Alhasan, A. H.; Mirkin, C. A. Selective Enhancement of Nucleases by Polyvalent DNA-Functionalized Gold Nanoparticles. J. Am. Chem. Soc. 2011, 133, 2120-2123.

(136) Demers, L. M.; Mirkin, C. A.; Mucic, R. C.; Reynolds, R. A.; Letsinger, R. L.; Elghanian, R.; Viswanadham, G. A Fluorescence-Based Method for Determining the Surface Coverage and Hybridization Efficiency of Thiol-Capped Oligonucleotides Bound to Gold Thin Films and Nanoparticles. Anal. Chem. 2000, $72,5535-5541$.

(137) Henry, M. R.; Wilkins Stevens, P.; Sun, J.; Kelso, D. M. Real-Time Measurements of DNA Hybridization on Microparticles with Fluorescence Resonance Energy Transfer. Anal. Biochem. 1999, 276, 204-214. 
(138) Thaxton, C. S.; Hill, H. D.; Georganopoulou, D. G.; Stoeva, S. I.; Mirkin, C. A. A Bio-Bar-Code Assay Based upon Dithiothreitol-Induced Oligonucleotide Release. Anal. Chem. 2005, 77, 8174-8178.

(139) Cleland, W. W. Dithiothreitol, a New Protective Reagent for SH Groups. Biochemistry 1964, 3, 480-482.

(140) Kim, E.-Y.; Stanton, J.; Vega, R. A.; Kunstman, K. J.; Mirkin, C. A.; Wolinsky, S. M. A Real-Time PCR-Based Method for Determining the Surface Coverage of Thiol-Capped Oligonucleotides Bound onto Gold Nanoparticles. Nucleic Acids Res. 2006, 34, e54.

(141) Liu, C.-W.; Huang, C.-C.; Chang, H.-T. Control over Surface DNA Density on Gold Nanoparticles Allows Selective and Sensitive Detection of Mercury(II). Langmuir 2008, 24, 8346-8350.

(142) Paliwoda, R. E.; Li, F.; Reid, M. S.; Lin, Y.; Le, X. C. Sequential Strand Displacement Beacon for Detection of DNA Coverage on Functionalized Gold Nanoparticles. Anal. Chem. 2014, 86, 6138-6143.

(143) Yu, H.; Xu, X.; Liang, P.; Loh, K. Y.; Guntupalli, B.; Roncancio, D.; Xiao, Y. A Broadly Applicable Assay for Rapidly and Accurately Quantifying DNA Surface Coverage on Diverse Particles. Bioconjug. Chem. 2017, 28, 933-943.

(144) Liu, J.; Lu, Y. Fast Colorimetric Sensing of Adenosine and Cocaine Based on a General Sensor Design Involving Aptamers and Nanoparticles. Angew. Chem., Int. Ed. 2006, 45, 90-94.

(145) Zhang, J.; Wang, L.; Pan, D.; Song, S.; Boey, F. Y. C.; Zhang, H.; Fan, C. Visual Cocaine Detection with Gold Nanoparticles and Rationally Engineered Aptamer Structures. Small 2008, 4, 1196-1200.

(146) Xia, F.; Zuo, X.; Yang, R.; Xiao, Y.; Kang, D.; Vallée-Bélisle, A.; Gong, X.; Yuen, J. D.; Hsu, B. B. Y.; Heeger, A. J.; et al. Colorimetric Detection of DNA, Small Molecules, Proteins, and Ions Using Unmodified Gold Nanoparticles and Conjugated Polyelectrolytes. Proc. Natl. Acad. Sci. U. S. A. 2010, 107, 1083710841.

(147) Li, H.; Rothberg, L. Colorimetric Detection of DNA Sequences Based on Electrostatic Interactions with Unmodified Gold Nanoparticles. Proc. Natl. Acad. Sci. U. S. A. 2004, 101, 14036-14039.

(148) Li, H.; Rothberg, L. J. Label-Free Colorimetric Detection of Specific Sequences in Genomic DNA Amplified by the Polymerase Chain Reaction. J. Am. Chem. Soc. 2004, 126, 10958-10961. 
(149) Mirau, P. A.; Smith, J. E.; Chávez, J. L.; Hagen, J. A.; Kelley-Loughnane, N.; Naik, R. Structured DNA Aptamer Interactions with Gold Nanoparticles. Langmuir 2018, 34, 2139-2146.

(150) Zhao, W.; Chiuman, W.; Lam, J. C. F.; McManus, S. A.; Chen, W.; Cui, Y.; Pelton, R.; Brook, M. A.; Li, Y. DNA Aptamer Folding on Gold Nanoparticles: From Colloid Chemistry to Biosensors. J. Am. Chem. Soc. 2008, 130, 3610-3618.

(151) Elghanian, R.; Storhoff, J. J.; Mucic, R. C.; Letsinger, R. L.; Mirkin, C. A. Selective Colorimetric Detection of Polynucleotides Based on the DistanceDependent Optical Properties of Gold Nanoparticles. Science 1997, 277, 10781081.

(152) Zhao, W.; Lam, J. C. F.; Chiuman, W.; Brook, M. A.; Li, Y. Enzymatic Cleavage of Nucleic Acids on Gold Nanoparticles: A Generic Platform for Facile Colorimetric Biosensors. Small 2008, 4, 810-816.

(153) Liu, T.; Zhao, J.; Zhang, D.; Li, G. Novel Method to Detect DNA Methylation Using Gold Nanoparticles Coupled with Enzyme-Linkage Reactions. Anal. Chem. 2009, 82, 229-233.

(154) Wu, S.; Liang, P. P.; Yu, H. X.; Xu, X. W.; Liu, Y.; Lou, X. H.; Xiao, Y. Amplified Single Base-Pair Mismatch Detection via Aggregation of ExonucleaseSheared Gold Nanoparticles. Anal. Chem. 2014, 86, 3461-3467.

(155) Liang, P.; Canoura, J.; Yu, H.; Alkhamis, O.; Xiao, Y. Dithiothreitol-Regulated Coverage of Oligonucleotide-Modified Gold Nanoparticles To Achieve Optimized Biosensor Performance. ACS Appl. Mater. Interfaces 2018, 10, 4233-4242.

(156) Rogers, K. R. Principles of Affinity-Based Biosensors. Mol. Biotechnol. 2000, 14, 109-129.

(157) Duan, R. X.; Lou, X. D.; Xia, F. The Development of Nanostructure Assisted Isothermal Amplification in Biosensors. Chem. Soc. Rev. 2016, 45, 1738-1749.

(158) Li, J. J.; Chu, Y.; Lee, B. Y.-H.; Xie, X. S. Enzymatic Signal Amplification of Molecular Beacons for Sensitive DNA Detection. Nucleic Acids Res. 2008, 36, e36.

(159) Gerasimova, Y. V.; Kolpashchikov, D. M. Enzyme-Assisted Target Recycling (EATR) for Nucleic Acid Detection. Chem. Soc. Rev. 2014, 43, 6405-6438.

(160) Liu, X. Q.; Freeman, R.; Willner, I. Amplified Fluorescence Aptamer-Based Sensors Using Exonuclease III for the Regeneration of the Analyte. Chem. - A Eur. J. 2012, 18, 2207-2211. 
(161) Gerasimova, Y. V.; Peck, S.; Kolpashchikov, D. M. Enzyme-Assisted Binary Probe for Sensitive Detection of RNA and DNA. Chem. Commun. 2010, 46, 87618763.

(162) Wu, D.; Yin, B. C.; Ye, B. C. A Label-Free Electrochemical DNA Sensor Based on Exonuclease III-Aided Target Recycling Strategy for Sequence-Specific Detection of Femtomolar DNA. Biosens. Bioelectron. 2011, 28, 232-238.

(163) Lyamichev, V.; Mast, A. L.; Hall, J. G.; Prudent, J. R.; Kaiser, M. W.; Takova, T.; Kwiatkowski, R. W.; Sander, T. J.; de Arruda, M.; Arco, D. A.; et al. Polymorphism Identification and Quantitative Detection of Genomic DNA by Invasive Cleavage of Oligonucleotide Probes. Nat. Biotechnol. 1999, 17, 292-296.

(164) Zuo, X. L.; Xia, F.; Xiao, Y.; Plaxco, K. W. Sensitive and Selective Amplified Fluorescence DNA Detection Based on Exonuclease III-Aided Target Recycling. J. Am. Chem. Soc. 2010, 132, 1816-1818.

(165) Riezzo, I.; Fiore, C.; De Carlo, D.; Pascale, N.; Neri, M.; Turillazzi, E.; Fineschi, V. Side Effects of Cocaine Abuse: Multiorgan Toxicity and Pathological Consequences. Curr. Med. Chem. 2012, 19, 5624-5646.

(166) Drug Enforcement Administration. NFLIS-Drug 2017 Annual Report; 2018.

(167) Center for Behavioral Health Statistics and Quality. Key Substance Use and Mental Health Indicators in the United States: Results from the 2015 National Survey on Drug Use and Health; 2016.

(168) Longcope, C. Dehydroepiandrosterone Metabolism. J. Endocrinol. 1996, 150, S125-127.

(169) Orentreich, N.; Brind, J. L.; Rizer, R. L.; Vogelman, J. H. Age Changes and Sex Differences in Serum Dehydroepiandrosterone Sulfate Concentrations throughout Adulthood. J. Clin. Endocrinol. Metab. 1984, 59, 551-555.

(170) Straub, R. H.; Weidler, C.; Demmel, B.; Herrmann, M.; Kees, F.; Schmidt, M.; Schölmerich, J.; Schedel, J. Renal Clearance and Daily Excretion of Cortisol and Adrenal Androgens in Patients with Rheumatoid Arthritis and Systemic Lupus Erythematosus. Ann. Rheum. Dis. 2004, 63, 961-968.

(171) Strahm, E.; Kohler, I.; Rudaz, S.; Martel, S.; Carrupt, P. A.; Veuthey, J. L.; Saugy, M.; Saudan, C. Isolation and Quantification by High-Performance Liquid Chromatography-Ion-Trap Mass Spectrometry of Androgen Sulfoconjugates in Human Urine. J. Chromatogr. A 2008, 1196-1197, 153-160.

(172) MASON, H. L.; KEPIER, E. J. Isolation of Steroids from the Urine of Patients with Adrenal Cortical Tumors and Adrenal Cortical Hyperplasia; a New 17- 
Ketosteroid, Androstane-3 (Alpha) 11-Diol-17-One. J. Biol. Chem. 1945, 161, 235-257.

(173) Banks, M. L.; Worst, T. J.; Rusyniak, D. E.; Sprague, J. E. Synthetic Cathinones ("Bath Salts"). J. Emerg. Med. 2014, 46, 632-642.

(174) Spiller, H. A.; Ryan, M. L.; Weston, R. G.; Jansen, J. Clinical Experience with and Analytical Confirmation of "Bath Salts" and "Legal Highs" (Synthetic Cathinones) in the United States. Clin. Toxicol. 2011, 49, 499-505.

(175) Prosser, J. M.; Nelson, L. S. The Toxicology of Bath Salts: A Review of Synthetic Cathinones. J. Med. Toxicol. 2012, 8, 33-42.

(176) Weinstein, A. M.; Rosca, P.; Fattore, L.; London, E. D. Synthetic Cathinone and Cannabinoid Designer Drugs Pose a Major Risk for Public Health. Front. Psychiatry 2017, 8, 156.

(177) USDOJ: Drug Enforcement Administration. Part 1308 -- Schedules of Controlled Substances. In CFR - Code of Federal Regulations Title 21; 2017.

(178) Perutz, M. F.; Wilkinson, A. J.; Paoli, M.; Dodson, G. G. The Stereochemical Mechanism of the Cooperative Effects in Hemoglobin Revisited. Annu. Rev. Biophys. Biomol. Struct. 1998, 27, 1-34.

(179) Meyer, T.; Holowka, D.; Stryer, L. Highly Cooperative Opening of Calcium Channels by Inositol 1,4,5-Trisphosphate. Science 1988, 240, 653-656.

(180) Krell, T.; Terán, W.; Mayorga, O. L.; Rivas, G.; Jiménez, M.; Daniels, C.; MolinaHenares, A. J.; Martínez-Bueno, M.; Gallegos, M. T.; Ramos, J. L. Optimization of the Palindromic Order of the TtgR Operator Enhances Binding Cooperativity. $J$. Mol. Biol. 2007, 369, 1188-1199.

(181) Bray, D. Protein Molecules as Computational Elements in Living Cells. Nature 1995, 376, 307-312.

(182) Mandal, M.; Lee, M.; Barrick, J. E.; Weinberg, Z.; Emilsson, G. M.; Ruzzo, W. L.; Breaker, R. R. A Glycine-Dependent Riboswitch That Uses Cooperative Binding to Control Gene Expression. Science 2004, 306, 275-279.

(183) Sudarsan, N.; Hammond, M. C.; Block, K. F.; Welz, R.; Barrick, J. E.; Roth, A.; Breaker, R. R. Tandem Riboswitch Architectures Exhibit Complex Gene Control Functions. Science 2006, 314, 300-304.

(184) Jose, A. M.; Soukup, G. A.; Breaker, R. R. Cooperative Binding of Effectors by an Allosteric Ribozyme. Nucleic Acids Res. 2001, 29, 1631-1637. 
(185) Simon, A. J.; Vallée-Bélisle, A.; Ricci, F.; Watkins, H. M.; Plaxco, K. W. Using the Population-Shift Mechanism to Rationally Introduce "Hill-Type" Cooperativity into a Normally Non-Cooperative Receptor. Angew. Chemie Int. Ed. 2014, 53, 9471-9475.

(186) Simon, A. J.; Vallée-Bélisle, A.; Ricci, F.; Plaxco, K. W. Intrinsic Disorder as a Generalizable Strategy for the Rational Design of Highly Responsive, Allosterically Cooperative Receptors. Proc. Natl. Acad. Sci. U. S. A. 2014, 111, 15048-15053.

(187) Scientific Working Group for Forensic Toxicology. Scientific Working Group for Forensic Toxicology (SWGTOX) Standard Practices for Method Validation in Forensic Toxicology. J. Anal. Toxicol. 2013, 37, 452-474.

(188) Sato, Y.; Kageyama, T.; Nishizawa, S.; Teramae, N. Competitive Binding of Abasic Site-Binding Ligands and Masking Ligands to DNA Duplexes for the Analysis of Single-Base Mutation. Anal. Sci. 2013, 29, 15-19.

(189) Sato, Y.; Nishizawa, S.; Yoshimoto, K.; Seino, T.; Ichihashi, T.; Morita, K.; Teramae, N. Influence of Substituent Modifications on the Binding of 2-Amino1,8-Naphthyridines to Cytosine Opposite an AP Site in DNA Duplexes: Thermodynamic Characterization. Nucleic Acids Res. 2009, 37, 1411-1422.

(190) Reinstein, O.; Yoo, M.; Han, C.; Palmo, T.; Beckham, S. A.; Wilce, M. C. J.; Johnson, P. E. Quinine Binding by the Cocaine-Binding Aptamer. Thermodynamic and Hydrodynamic Analysis of High-Affinity Binding of an Off-Target Ligand. Biochemistry 2013, 52, 8652-8662.

(191) Owczarzy, R.; Moreira, B. G.; You, Y.; Behlke, M. A.; Walder, J. A. Predicting Stability of DNA Duplexes in Solutions Containing Magnesium and Monovalent Cations. Biochemistry 2008, 47, 5336-5353.

(192) Hill, A. V. The Possible Effects of the Aggregation of the Molecules of Haemoglobin on Its Dissociation Curves. J. Physiol. 1910, 40, iv-vii.

(193) Daranas, W. B. T. and A. H. On the Value of C: Can Low Affinity Systems Be Studied by Isothermal Titration Calorimetry. J. Am. Chem. Soc. 2003, 125, 1485914866.

(194) Freyer, M. W.; Lewis, E. A. Isothermal Titration Calorimetry: Experimental Design, Data Analysis, and Probing Macromolecule/Ligand Binding and Kinetic Interactions. Methods Cell Biol. 2008, 84, 79-113.

(195) Dahlquist, F. W. The Meaning of Scatchard and Hill Plots. Methods Enzymol. 1978, 48, 270-299. 
(196) Johansson, M. K. Choosing Reporter-Quencher Pairs for Efficient Quenching through Formation of Intramolecular Dimers. Methods Mol. Biol. 2006, 335, 1729.

(197) Gjerde, H.; Langel, K.; Favretto, D.; Verstraete, A. G. Estimation of Equivalent Cutoff Thresholds in Blood and Oral Fluid for Drug Prevalence Studies. J. Anal. Toxicol. 2014, 38, 92-98.

(198) Schramm, W.; Craig, P. A.; Smith, R. H.; Berger, G. E. Cocaine and Benzoylecgonine in Saliva, Serum, and Urine. Clin. Chem. 1993, 39, 481-487.

(199) Ma, D.-L.; Wang, M.; He, B.; Yang, C.; Wang, W.; Leung, C.-H. A Luminescent Cocaine Detection Platform Using a Split G-Quadruplex-Selective Iridium(III) Complex and a Three-Way DNA Junction Architecture. ACS Appl. Mater. Interfaces 2015, 7, 19060-19067.

(200) Shi, Y.; Dai, H. C.; Sun, Y. J.; Hu, J. T.; Ni, P. J.; Li, Z. Fluorescent Sensing of Cocaine Based on a Structure Switching Aptamer, Gold Nanoparticles and Graphene Oxide. Analyst 2013, 138, 7152-7156.

(201) Du, Y.; Chen, C. G.; Yin, J. Y.; Li, B. L.; Zhou, M.; Dong, S. J.; Wang, E. K. Solid-State Probe Based Electrochemical Aptasensor for Cocaine: A Potentially Convenient, Sensitive, Repeatable, and Integrated Sensing Platform for Drugs. Anal. Chem. 2010, 82, 1556-1563.

(202) Cone, E. J.; Hillsgrove, M.; Darwin, W. D. Simultaneous Measurement of Cocaine, Cocaethylene, Their Metabolites, and "Crack" Pyrolysis Products by Gas Chromatography-Mass Spectrometry. Clin. Chem. 1994, 40, 1299-1305.

(203) Freeman, R.; Sharon, E.; Tel-Vered, R.; Willner, I. Supramolecular CocaineAptamer Complexes Activate Biocatalytic Cascades. J. Am. Chem. Soc. 2009, 131, 5028-5029.

(204) Lu, C.-H.; Wang, F.; Willner, I. Amplified Optical Aptasensors through the Endonuclease-Stimulated Regeneration of the Analyte. Chem. Sci. 2012, 3, 26162622.

(205) Li, Q.; Wang, Y. D.; Shen, G. L.; Tang, H.; Yu, R. Q.; Jiang, J. H. Split Aptamer Mediated Endonuclease Amplification for Small-Molecule Detection. Chem. Commun. 2015, 51, 4196-4199.

(206) Niu, S. Y.; Lou, X. F.; Jiang, Y.; Lin, J. H. A Novel Fluorescence Sensor for Cocaine with Signal Amplification through Cycling Exo-Cleaving with a Hairpin Probe. Anal. Lett. 2012, 45, 1919-1927. 
(207) Zhu, Z.; Wu, C.; Liu, H.; Zou, Y.; Zhang, X.; Kang, H.; Yang, C. J.; Tan, W. An Aptamer Cross-Linked Hydrogel as a Colorimetric Platform for Visual Detection. Angew. Chem., Int. Ed. 2010, 49, 1052-1056.

(208) Citartan, M.; Gopinath, S. C. B.; Tominaga, J.; Tan, S.-C.; Tang, T.-H. Assays for Aptamer-Based Platforms. Biosens. Bioelectron. 2012, 34, 1-11.

(209) Zheng, D. M.; Zou, R. X.; Lou, X. H. Label-Free Fluorescent Detection of Ions, Proteins, and Small Molecules Using Structure-Switching Aptamers, SYBR Gold, and Exonuclease I. Anal. Chem. 2012, 84, 3554-3560.

(210) Biebricher, A. S.; Heller, I.; Roijmans, R. F. H.; Hoekstra, T. P.; Peterman, E. J. G.; Wuite, G. J. L. The Impact of DNA Intercalators on DNA and DNAProcessing Enzymes Elucidated through Force-Dependent Binding Kinetics. Nat. Commun. 2015, 6, 7304.

(211) Kang, S.; Ohshima, K.; Shimizu, M.; Amirhaeri, S.; Wells, R. D. Pausing of DNA Synthesis in Vitro at Specific Loci in CTG and CGG Triplet Repeats from Human Hereditary Disease Genes. J. Biol. Chem. 1995, 270, 27014-27021.

(212) Tian, L. G.; Sayer, J. M.; Kroth, H.; Kalena, G.; Jerina, D. M.; Shuman, S. Benzo[a]Pyrene-DG Adduct Interference Illuminates the Interface of Vaccinia Topoisomerase with the DNA Minor Groove. J. Biol. Chem. 2003, 278, 99059911.

(213) Pierce, J. R.; Nazimiec, M.; Tang, M. S. Comparison of Sequence Preference of Tomaymycin- and Anthramycin-DNA Bonding by Exonuclease III and .Lambda. Exonuclease Digestion and UvrABC Nuclease Incision Analysis. Biochemistry 1993, 32, 7069-7078.

(214) Takeuchi, M.; Lillis, R.; Demple, B.; Takeshita, M. Interactions of Escherichia Coli Endonuclease IV and Exonuclease III with Abasic Sites in DNA. J. Biol. Chem. 1994, 269, 21907-21914.

(215) Shida, T.; Noda, M.; Sekiguchi, J. Cleavage of Single- and Double-Stranded DNAs Containing an Abasic Residue by Escherichia Coli Exonuclease III (AP Endonuclease VI). Nucleic Acids Res. 1996, 24, 4572-4576.

(216) Hoheisel, J. D. On the Activities of Escherichia Coli Exonuclease III. Anal. Biochem. 1993, 209, 238-246.

(217) Link, S.; El-Sayed, M. A. Size and Temperature Dependence of the Plasmon Absorption of Colloidal Gold Nanoparticles. J. Phys. Chem. B 1999, 103, 42124217. 
(218) Parak, W. J.; Pellegrino, T.; Micheel, C. M.; Gerion, D.; Williams, S. C.; Alivisatos, A. P. Conformation of Oligonucleotides Attached to Gold Nanocrystals Probed by Gel Electrophoresis. Nano Lett. 2003, 3, 33-36.

(219) Wu, J.; Campuzano, S.; Halford, C.; Haake, D. A.; Wang, J. Ternary Surface Monolayers for Ultrasensitive (Zeptomole) Amperometric Detection of Nucleic Acid Hybridization without Signal Amplification. Anal. Chem. 2010, 82, 88308837.

(220) Cederquist, K. B.; Keating, C. D. Hybridization Efficiency of Molecular Beacons Bound to Gold Nanowires: Effect of Surface Coverage and Target Length. Langmuir 2010, 26, 18273-18280.

(221) de Souza, L. M.; Rodrigues, R. R. T.; Santos, H.; Costa, H. B.; Merlo, B. B.; Filgueiras, P. R.; Poppi, R. J.; Vaz, B. G.; Romão, W. A Survey of Adulterants Used to Cut Cocaine in Samples Seized in the Espírito Santo State by GC-MS Allied to Chemometric Tools. Sci. Justice 2016, 56, 73-79.

(222) Peterson, A. M.; Jahnke, F. M.; Heemstra, J. M. Modulating the Substrate Selectivity of DNA Aptamers Using Surfactants. Langmuir 2015, 31, 1176911773.

(223) Crooks, G. E.; Hon, G.; Chandonia, J.-M. M.; Brenner, S. E. WebLogo: A Sequence Logo Generator. Genome Res. 2004, 14, 1188-1190.

(224) Choi, J. K.; D’Urso, A.; Trauernicht, M.; Shabbir-Hussain, M.; Holmes, A. E.; Balaz, M. 3,3'-Diethylthiatricarbocyanine Iodide: A Highly Sensitive Chiroptical Reporter of DNA Helicity and Sequence. Int. J. Mol. Sci. 2011, 12, 8052-8062.

(225) Garoff, R. A.; Litzinger, E. A.; Connor, R. E.; Fishman, I.; Armitage, B. A. Helical Aggregation of Cyanine Dyes on DNA Templates: Effect of Dye Structure on Formation of Homo- and Heteroaggregates. Langmuir 2002, 18, 6330-6337.

(226) Seifert, J. L.; Connor, R. E.; Kushon, S. A.; Wang, M.; Armitage, B. A. Spontaneous Assembly of Helical Cyanine Dye Aggregates on DNA Nanotemplates. J. Am. Chem. Soc. 1999, 121, 2987-2995.

(227) Zangi, R.; Hagen, M.; Berne, B. J. Effect of Ions on the Hydrophobic Interaction between Two Plates. J. Am. Chem. Soc. 2007, 129, 4678-4686.

(228) Schirmer, R. E. UV and Visible Absorption Techniques. In Modern Methods of Pharmaceutical Analysis; Schirmer, R. E., Ed.; CRC Press: Boca Raton, 1982; pp 31-124.

(229) Guminetsky, S. G.; Gayka, O. R.; Kokoschuk, G. I.; Grigorishin, P. M.; Kirsh, N. L. Absorption Spectra of the Main Organic Components of Human Urine in the 
Absence of Proteins; Angelsky, O. V., Ed.; International Society for Optics and Photonics, 1999; Vol. 3904, p 579.

(230) St John, A.; Price, C. P. Existing and Emerging Technologies for Point-of-Care Testing. Clin. Biochem. Rev. 2014, 35, 155-167.

(231) Zhang, H.; Wang, S. Review on Enzyme-Linked Immunosorbent Assays for Sulfonamide Residues in Edible Animal Products. J. Immunol. Methods 2009, 350, $1-13$.

(232) Spinks, C. A. Broad-Specificity Immunoassay of Low Molecular Weight Food Contaminants: New Paths to Utopia! Trends Food Sci. Technol. 2000, 11, 210217.

(233) Slavkovic, S.; Altunisik, M.; Reinstein, O.; Johnson, P. E. Structure-Affinity Relationship of the Cocaine-Binding Aptamer with Quinine Derivatives. Bioorg. Med. Chem. 2015, 23, 2593-2597.

(234) Martin, M. Cutadapt Removes Adapter Sequences from High-Throughput Sequencing Reads. EMBnet.journal 2011, 17, 10.

(235) Alam, K. K.; Chang, J. L.; Burke, D. H. FASTAptamer: A Bioinformatic Toolkit for High-Throughput Sequence Analysis of Combinatorial Selections. Mol. Ther. Nucleic Acids 2015, 4, e230.

(236) Ellefsen, K. N.; Anizan, S.; Castaneto, M. S.; Desrosiers, N. A.; Martin, T. M.; Klette, K. L.; Huestis, M. A. Validation of the Only Commercially Available Immunoassay for Synthetic Cathinones in Urine: Randox Drugs of Abuse V Biochip Array Technology. Drug Test. Anal. 2014, 6, 728-738.

(237) Ellefsen, K. N.; Concheiro, M.; Huestis, M. A. Synthetic Cathinone Pharmacokinetics, Analytical Methods, and Toxicological Findings from Human Performance and Postmortem Cases. Drug Metab. Rev. 2016, 48, 237-265.

(238) Amaratunga, P.; Lemberg, B. L.; Lemberg, D. Quantitative Measurement of Synthetic Cathinones in Oral Fluid. J. Anal. Toxicol. 2013, 37, 622-628.

(239) Mohamed, K. M.; Al-Hazmi, A. H.; Alasiri, A. M.; Ali, M. E.-S. A GC-MS Method for Detection and Quantification of Cathine, Cathinone, Methcathinone and Ephedrine in Oral Fluid. J. Chromatogr. Sci. 2016, 54, 1271-1276. 
VITA

\section{HAIXIANG YU}

\section{EDUCATION}

Born, Nanjing, China

2009-2013

B.S., Biotechnology

China Pharmaceutical University

Nanjing University

Nanjing, China

2013-2014

Teaching Assistant

Florida International University

Miami, FL

2014-2015

Research Assistant

Florida International University

Miami, FL

2015-2018

NIJ graduate research fellow, Florida International University

Miami, FL

2018-2019

Dissertation Year Fellowship Award, Florida International University

Miami, FL

\section{SELECTED PUBLICATIONS}

Yang W.J., Yu H.X., Alkhamis O., Liu Y.Z., Canoura, J., Fu F.F. \& Xiao Y. (2019) In vitro isolation of class-specific oligonucleotide-based small-molecule receptors. Nucleic Acids Res., gkz224.

Canoura J., Wang Z.W., Yu H.X., Alkhamis O., Fu F.F. \& Xiao Y. (2018) No structureswitching required: a generalizable exonuclease-mediated aptamer-based assay for smallmolecule detection. J. Am. Chem. Soc., 140, 9961 - 9971.

Wang Z.W., Yu H.X., Canoura J., Liu Y.Z., Alkhamis O., Fu F.F. \& Xiao Y. (2018) Introducing structure-switching functionality into small-molecule-binding aptamers via nuclease-directed truncation. Nucleic Acids Res., 46, e81. 
Yu H.X., Yang W.J., Alkhamis O., Canoura J., Yang K. \& Xiao Y. (2018) In vitro isolation of small-molecule-binding aptamers with intrinsic dye-displacement functionality. Nucleic Acids Res., 46, e43.

Liang P.P., Canoura J.; Yu H.X., Alkhamis O. \& Xiao Y. (2018) Dithiothreitol-regulated coverage of oligonucleotide-modified gold nanoparticles to achieve optimized biosensor performance. ACS Appl. Mater. Interfaces., 10, 4233 - 4242.

Yu H.X., Canoura J., Guntupalli B., Alkhamis O. \& Xiao Y. (2018) Sensitive detection of small-molecule targets using cooperative binding split aptamers and enzyme-assisted target recycling. Anal. Chem., 90, $1748-1758$.

Yu H.X., Xu X.W., Liang P.P., Loh K.Y., Guntupalli B., Roncancio D. \& Xiao Y. (2017) A broadly-applicable assay for rapidly and accurately quantifying DNA surface coverage on diverse particles. Bioconjugate Chem., 28, 933 - 943.

Yu H.X., Canoura J., Guntupalli B., Lou X.H. \& Xiao Y. (2017) A cooperative-binding split aptamer assay for rapid, specific and ultra-sensitive fluorescence detection of cocaine in saliva. Chem. Sci., 8, 131-141.

Xu Q., Lou X.H., Wang L., Ding X.F., Yu H.X. \& Xiao Y. (2016) Rapid, surfactant-free, and quantitative functionalization of gold nanoparticles with thiolated DNA under physiological $\mathrm{pH}$ and its application in molecular beacon-based biosensor. ACS Appl. Mater. Interfaces. 8, $27298-27304,2016$.

Zhao T., Liu R., Ding X.F., Zhao J.C., Yu H.X., Wang X., Lou X.H., He M. \& Xiao Y. (2015) Nanoprobe-enhanced, split aptamer-based electrochemical sandwich assay for ultrasensitive detection of small molecules. Anal. Chem. 87, 7712 - 7719.

Guntupalli B., Liang P.P., Lee J.H., Yang Y.H., Yu H.X., Canoura J., He J., Li W.Z., Weizmann Y. \& Xiao Y. (2015) Ambient filtration method to rapidly prepare highly conductive, paper-based porous gold films for electrochemical biosensing. ACS Appl. Mater. Interfaces. 10, $27049-27058$.

Liang P.P., Yu H.X., Guntupalli B. \& Xiao Y. (2015) Paper-based device for rapid visualization of NADH based on dissolution of gold nanoparticles. ACS Appl. Mater. Interfaces. 7, $15023-15030$.

Roncancio D., Yu H.X., Xu X.W., Wu S., Liu R., Debord J., Lou X.H. \& Xiao Y. (2014) A label-free aptamer-fluorophore assembly for rapid and specific detection of cocaine in biofluids. Anal. Chem. 86, $11100-11106$.

Wu S., Liang P.P., Yu H.X., Xu X.W., Liu Y., Lou X.H. \& Xiao Y. (2014) Amplified single base-pair mismatch detection via the aggregation of exonuclease-sheared gold nanoparticles. Anal. Chem. 86, $3461-3467$. 